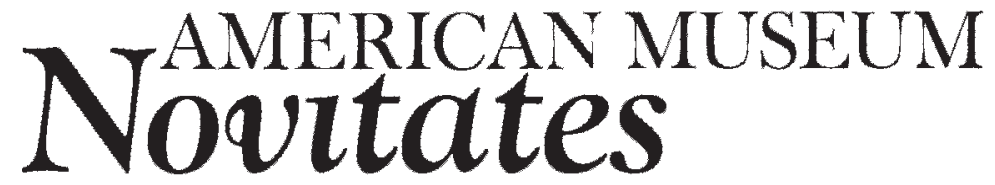

PUBLISHED BY THE AMERICAN MUSEUM OF NATURAL HISTORY CENTRAL PARK WEST AT 79TH STREET, NEW YORK, NY 10024 Number 3627, 58 pp., 8 figures, 7 tables

\title{
Additional Distributional Records of Ambystoma laterale, A. jeffersonianum (Amphibia: Caudata) and Their Unisexual Kleptogens in Northeastern North America
}

\author{
JAMES P. BOGART ${ }^{1}$ AND MICHAEL W. KLEMENS ${ }^{2}$
}

\begin{abstract}
Several species of mole salamanders in the genus Ambystoma are targeted by various state, provincial, and federal agencies for conservation. These salamanders have specific wetland and forested upland habitat requirements that render them vulnerable to environmental alteration. The blue-spotted salamander, Ambystoma laterale (LL) and the Jefferson salamander, A. jeffersonianum (JJ) have both been listed for protection in various parts of their ranges, but the identification of these salamanders is confusing because they often coexist with unisexual individuals that are mostly polyploid and use the sexual species as sperm donors. We used isozyme electrophoresis, blood erythrocytes, and chromosome counts in a continued effort to identify sexual and unisexual individuals in eastern North America. We examined 1377 salamanders from 118 sites in Connecticut, Massachusetts, New Jersey, New York, Pennsylvania, and Virginia. Most Pennsylvania salamanders were $A$. jeffersonianum (JJ) but $A$. laterale (LL), previously unknown from Pennsylvania, were found in that state. The two sexual species were never found together. We found diploid (LJ), triploid (LLJ; LJJ), and tetraploid (LLLJ; LJJJ; LLJJ) unisexuals. At most collecting sites, unisexuals were more numerous than sexual individuals. The association of sexual and unisexual individuals support a kleptogenic reproductive system in which the unisexuals steal genomes from their sympatric sexual sperm donors.
\end{abstract}

\footnotetext{
${ }^{1}$ Department of Integrative Biology, University of Guelph, Guelph, Ontario, Canada N1G 2W1 (jbogart@uoguelph.ca).

${ }^{2}$ Division of Vertebrate Zoology, Herpetology, American Museum of Natural History; Cary Institute of Ecosystem Studies, P.O. Box AB, 2801 Sharon Turnpike, Millbrook, NY 12545 (klemensm@ecostudies.org).
} 
TABLE 1

Conservation status of Ambystoma laterale and $A$. jeffersonianum populations in Connecticut, Massachusetts, New Jersey, New York, Pennsylvania, and Rhode Island $\mathrm{E}=$ endangered, $\mathrm{T}=$ threatened, $\mathrm{SC}=$ special concern.

\begin{tabular}{|c|c|c|c|c|c|c|}
\hline $\begin{array}{c}\text { Genetic composition } \\
\text { of individual } \\
\text { breeding site }\end{array}$ & CT & MA & NJ & NY & PA & RI \\
\hline $\begin{array}{l}\text { A. jeffersonianum } \\
\text { complex }\end{array}$ & $\mathrm{SC}$ & $\mathrm{SC}$ & $\mathrm{SC}$ & $\mathrm{SC}$ & $\begin{array}{l}\text { No conservation status (not } \\
\text { considered native to PA) }\end{array}$ & $\begin{array}{l}\text { Not known } \\
\text { from RI }\end{array}$ \\
\hline A. jeffersonianum & $\begin{array}{l}\text { Not known } \\
\text { from } \mathrm{CT}^{\mathrm{a}}\end{array}$ & $\begin{array}{l}\text { Not known } \\
\text { from } \mathrm{MA}^{\mathrm{a}}\end{array}$ & $\begin{array}{l}\text { Not known } \\
\text { from } \mathrm{NJ}^{\mathrm{a}}\end{array}$ & $\mathrm{SC}$ & $\begin{array}{l}\text { No conservation status in } \\
\text { PA }\end{array}$ & $\begin{array}{l}\text { Not known } \\
\text { from } \mathrm{RI}^{\mathrm{a}}\end{array}$ \\
\hline A. laterale complex & $\mathrm{SC}$ & $\mathrm{SC}$ & $\mathrm{E}$ & $\mathrm{SC}$ & $\begin{array}{l}\text { No conservation status (not } \\
\text { considered native to PA) }\end{array}$ & $\begin{array}{l}\text { Not known } \\
\text { from } \mathrm{RI}^{\mathrm{a}}\end{array}$ \\
\hline A. laterale & $\mathrm{T}$ & $\mathrm{SC}^{\mathrm{d}}$ & $\mathrm{E}$ & $\mathrm{SC}^{\mathrm{d}}$ & $\begin{array}{c}\text { No conservation status (not } \\
\text { considered native to PA) }\end{array}$ & Extirpated $^{\mathrm{e}}$ \\
\hline
\end{tabular}

\footnotetext{
${ }^{\text {a }}$ Bogart and Klemens, 1997; Bogart and Klemens, present study.

${ }^{\mathrm{b}}$ This study confirms the presence of unisexuals associated with $A$. jeffersonianum in northeastern PA (Monroe Co.).

${ }^{\mathrm{c}}$ This study confirms the presence of $A$. laterale and associated unisexuals in northwestern PA (McKean Co.) and northeastern PA (Northampton Co.).

${ }^{\mathrm{d}}$ MA and NY do not recognize the unique conservation status of pure diploid $A$. laterale populations in southeastern MA and eastern Long Island, NY.

${ }^{\mathrm{e}}$ Pure diploid $A$. laterale was reported from the Pawtucket area of RI by Drowne (1905). These populations are considered extirpated (C. Raithel, personal commun.).
}

\section{INTRODUCTION}

There are about 30 living species of mole salamanders in the North American family Ambystomatidae (Petranka, 1998). Most species occur in the continental United States, but there has been a Mexican invasion of the Ambystoma tigrinum complex (Shaffer and McKnight, 1996) and some species have northern ranges that include parts of Canada (Petranka, 1998). Mole salamanders often have specific habitat requirements that make them vulnerable to anthropogenic environmental alterations and most species are listed as candidates for conservation in some or all parts of their range. The blue-spotted salamander, A. laterale and the Jefferson salamander, $A$. jeffersonianum present problems for conservationists because they both coexist with unisexual individuals that normally do not have a conservation status (Kraus, 1995). Connecticut lists Ambystoma jeffersonianum complex and $A$. laterale complex as state species of special concern because it is difficult to distinguish the unisexuals from the sexual species (Klemens, 2000). Connecticut also lists the pure diploid populations of $A$. laterale in the eastern portion of the state as a threatened species (Klemens, 2000). Connecticut is the only state in the northeastern United States that has differentially protected the pure diploid $A$. laterale populations that also occur in southeastern Massachusetts and on the eastern tip of Long Island, New York. The conservation status of these salamanders does vary over their ranges (table 1).

We (Bogart and Klemens, 1997) provided a detailed historical perspective on the unisexual salamanders and their associated sperm donor species. We also identified and characterized populations of $A$. laterale, $A$. jeffersonianum, and their unisexual associates in the New England states and parts of New York. Based on 1002 individuals from 106 sites, we found the unisexuals to be common and widespread. The majority of the populations that contained either $A$. laterale or $A$. jeffersonianum also contained unisexual individuals. Seventy percent of the salamanders in that study were found to be unisexuals. Ten of the 106 sites were found to contain only $A$. laterale, but six of these sites were represented by one or two individuals. Samples obtained from two of the three sites where only $A$. jeffersonianum were found consisted of single specimens and only three individuals were sampled from the other 
A. jeffersonianum site. Diploid, triploid, and tetraploid unisexuals were found throughout New England. Most unisexual salamanders were polyploid, but, because diploid unisexuals were found at 21 scattered sites, ploidy is not a dependable distinguishing character to separate unisexual and sexual individuals without corroborative data. Unisexual salamanders are expected to be female, but rare "unisexual" males were also found; thus, knowing that an individual is a male does not always confirm a sexual species identification.

Clarification of the evolution of unisexual salamanders and the reproductive mode of these females has been further elucidated using gene sequences and microsatellite DNA loci (Bogart, 2003; Bogart et al., 2007). Mitochondrial DNA analysis show that the unisexuals arose about three to four million years ago from a hybridization event involving a female that shared its most recent common ancestor to individuals of $A$. barbouri from Kentucky. Ambystoma barbouri has a current, restricted range in Ohio, Kentucky, and Tennessee and is a recently uncovered (Kraus and Petranka, 1989) sister species (Petranka, 1998; Niedzwiecki, 2005) to $A$. texanum. Subsequently, unisexuals rapidly dispersed in eastern North America using a reproductive mode that appears to be unique (Bogart et al., 2007). All unisexuals maintain a similar $A$. barbouri-like mitochondrial DNA but can incorporate and exchange nuclear genomes with sympatric males. Because unisexual females can steal genomes from a variety of sympatric males, the suggested reproductive mode is kleptogenesis (Bogart et al., 2007) and has given rise to at least 20 nuclear genomic combinations, or kleptogens, that are diploid, triploid, tetraploid, and even pentaploid (Bogart, 2003).

To expand the coverage of populations and to include more of the range of these salamanders in New Jersey, New York, and Pennsylvania the present study used isozyme electrophoresis to identify additional individuals of sexual $A$. laterale, $A$. jeffersonianum, and unisexual kleptogens that use these species as sperm donors. We also sampled new populations in New England and included a few samples from populations that were previously sampled by Bogart and Klemens (1997) to increase the sample size and to confirm our findings in previously sampled populations. Accurate data on the distribution of this salamander complex should assist conservation efforts to protect these salamanders. Such data are also necessary to explore the evolutionary significance of kleptogenesis.

\section{MATERIALS AND METHODS}

Methods were mostly identical to those used by Bogart and Klemens (1997). Salamanders were collected with dip nets and minnow traps from breeding ponds early in the spring, while crossing roads at night during spring rains, or from under rocks and logs during the day from March through October. Egg mass surveys (Bogart, 1982) were not done because data obtained from such surveys may not provide a random sample of the population as most eggs laid by unisexual females do not result in hatched larvae and many hatched larvae do not survive to transformation. In some cases, when adults were not collected from a breeding pond, advanced larvae were randomly collected using a dip net. They were maintained through transformation in the laboratory and the juveniles were considered to be representative of the population or site. Our present study is a continuation of the collections done for our previous study (Bogart and Klemens, 1997). Collections for the present study were conducted over a nineyear period, from 1996 to 2004. Collections were concentrated in New York, New Jersey, and Pennsylvania, but collections also included sites in Connecticut, Massachusetts, and Virginia. Salamanders (1377 individuals) from 118 sites (appendix 1) were collected and shipped to Guelph for identification. Salamanders were injected with colchicine ( 0.25 to $0.75 \mathrm{ml}$ of a $0.1 \mathrm{mg} / \mathrm{ml}$ solution) two days before they were killed by prolonged anesthesia in a 7\% solution of buffered $(\mathrm{pH}$ 7.0) tricaine methane sulfonate (MS222). The heart was exposed and blood was collected from the conus arteriosis in heparinized microhematocrit tubes for ploidy determination. The intestine, including the cloaca, was dissected from each individual, immersed in de-ionized water for $15 \mathrm{~min}$, fixed in 3:1 
TABLE 2

Presumptive structural gene loci examined in Ambystoma

\begin{tabular}{|c|c|c|c|}
\hline Locus (abbreviation) $^{\mathrm{a}}$ & EC No. ${ }^{b}$ & Tissue $^{c}$ & $\mathrm{Gel}^{\mathrm{d}}$ \\
\hline Aspartate aminotransferase $\left(\right.$ Aat $\left.-1^{\mathrm{a}}\right)\left(=\right.$ Got- $\left.1^{\mathrm{e}}\right)$ & 2.6 .1 .1 & HMS & 2 \\
\hline Aat $-2^{\mathrm{a}}\left(=\right.$ Got $\left.-2^{\mathrm{e}}\right)$ & 2.6.1.1 & $\mathrm{L}$ & 2 \\
\hline Isocitrate dehydrogenase $\left(\mathrm{Idh}-1^{\mathrm{a}}\right)(=\mathrm{ICD}-1)$ & 1.1 .1 .42 & HMS & 1 \\
\hline Lactate dehydrogenase $\left(\right.$ Ldh- $\left.1^{\mathrm{a}}\right)$ & 1.1.1.27 & HMS & 1 \\
\hline Ldh-2 & 1.1.1.27 & HMS & 1 \\
\hline Malate dehydrogenase $\left(\mathrm{Mdh}-1^{\mathrm{a}}\right)$ & 1.1.1.37 & HMS & 1 \\
\hline Mannose-6-phosphate isomerase (Mpil) (= PGDH) & 5.3 .1 .8 & $\mathrm{~L}$ & 1 \\
\hline Phosphoglucose isomerase (Pgi) (= GPI) & 5.3.1.9 & HMS & 2 \\
\hline Phosphogucomutase (Pgm-1) & 2.7.5.1 & $\mathrm{L}$ & 1 \\
\hline Pgm-2 & 2.7.5.1 & $\mathrm{L}$ & 1 \\
\hline Superoxide dismutase (Sod- $\left.1^{\mathrm{a}}\right)$ & 1.15 .1 .1 & $\mathrm{~L}$ & 1 \\
\hline
\end{tabular}

${ }^{\text {a }}$ Loci that were found to be most useful for distinguishing $A$. laterale, A. jeffersonianum, and associated unisexuals. The abbreviations and synonyms are those used in this and most previous electrophoretic studies of vertebrates.

${ }^{\mathrm{b}}$ Standardized enzyme-numbering system established by the nomenclature committee of the International Union of Biochemistry (IUBC, 1984).

${ }^{\mathrm{c}}$ Tissues used to resolve the enzyme systems were liver (L) or a combination of heart, skeletal muscle, and spleen (HMS).

${ }^{\mathrm{d}}$ The electrophoretic conditions for the gels were: (1) amine-citrate, gel, and tray buffer adjusted to pH 6.5 (Clayton and Tretiak, 1972) run for $3 \mathrm{hr}$ at 250 volts; (2) tris-citrate, gel buffer $\mathrm{pH}$ 6.7, and tray buffer $\mathrm{pH} 6.3$ (Selander et al., 1971) run 3 to $4 \mathrm{hr}$ at 150 volts.

${ }^{\mathrm{e}}$ Got (glutamate oxaloaceto-transaminase) is a synonym of aspartate aminotransferase (Aat). Previous studies on Ambystoma used this earlier enzyme designation (Got).

ethanol:acetic acid in $1.5 \mathrm{ml}$ Eppendorf microtubes, and stored at $-20^{\circ} \mathrm{C}$ for chromosome analyses. Tissues required for isozyme electrophoresis were liver and a combination of the heart, skeletal muscle, and spleen. The tissues were removed from freshly killed salamanders and stored with an equal volume of de-ionized water in $1.5 \mathrm{ml}$ Eppendorf microtubes in an ultracold freezer $\left(-80^{\circ} \mathrm{C}\right)$. After the tissues were removed, the specimens were preserved and deposited at the American Museum of Natural History (AMNH) (appendix 1).

\section{ISOZYME ELECTROPHORESIS}

Just prior to electrophoresis, the frozen tissues were ground using a sharp glass rod and spun for two minutes in a microcentrifuge. Filter-paper wicks were used to soak up a portion of the supernatant. The wicks were air dried on filter paper and inserted in starch gels. Horizontal starch-gel electrophoresis followed the procedures outlined by Selander et al. (1971), Bogart (1982), and Bogart and Klemens (1997). The buffer systems used were described by Selander et al. (1971) and Clayton and Tretiak (1972). Electrophoretic loci for each enzyme system were numbered on the gel from the most anodally migrating locus. As in previous studies (Bogart et al., 1987; Bogart, 1989; Bogart and Klemens, 1997), alleles, or allozymes, were designated by their relative mobilities compared with the mobility of the most common allele in $A$. laterale, which was assigned a mobility of 100 . We chose 11 of the 21 isozyme loci used by Bogart and Klemens (1997) in our present study. These loci proved to be most useful in identifying the sexual and unisexual individuals and included loci that demonstrated some homozygosity and reversals in our previous study. The loci that were assayed, buffer systems used, and the tissues examined are provided in table 2. Nine loci were dimeric enzymes that have been the most useful in visualizing staining intensities of the bands (dosage) for assessing genome composition in polyploid amphibians (Danzmann and Bogart, 1982). Lactate dehydrogenase is a tetrameric enzyme that also expresses differential staining of the heterotetrameric bands. The only monomeric enzyme, phosphoglucose mutase, often demonstrated different staining intensities for two loci (pgm-1; pgm-2) and was previously found to posses a few rare 
heterozygotes at pgm-2 in both sexual and unisexual salamanders (Lowcock and Bogart, 1989; Bogart and Klemens, 1997).

\section{Ploidy Determination}

A small drop of blood from the hematocrit tube, taken from every individual, was mixed with an approximately equal amount of diluted $\left(25 \mathrm{ml} \mathrm{H}_{2} \mathrm{O}\right.$ added to $\left.100 \mathrm{ml}\right)$ Hanks Balanced Salt Solution (Sigma) and photographed under phase-contrast and bright-field optics. The area of the blood cells was determined using a sonic digitizer on a rearprojection image of the negatives (Austin and Bogart, 1982). Six blood cells from each individual were measured to obtain an average erythrocyte area. During our investigation, a flow cytometric method (FCM) was found to be a more accurate method to determine ploidy and that method was introduced into our study in 2003 following the methods in Ramsden et al. (2006). Blood cell data provided the first clear indication of diploid and tetraploid unisexuals that may not be easily distinguished from triploids using only the electrophoretic patterns. Chromosome numbers were the absolute proof of ploidy in the salamanders and those data were required if a discrepancy existed between the electrophoretic genotype, blood cell area, or flow cytometric analyses. When necessary, chromosomes were obtained from the gut epithelial tissue using procedures outlined by Kezer and Sessions (1979) and Sessions (1982). Unstained, or conventionally stained, chromosomes of $A$. laterale and $A$. jeffersonianum are virtually indistinguishable (Taylor and Bogart, 1990), so the chromosomes obtained for most individuals and those observed in our previous study (Bogart and Klemens, 1997) could not be used to assign genomic constitution in the unisexuals. New chromosome methods that use fluorescent genomic probes and in situ hybridization clearly differentiate genomes of these two species in the unisexuals (Bi and Bogart, 2006). Microsatellite DNA loci that are amplified using primers developed for A. jeffersonianum (Julian et al., 2003) also proved to be an accurate method to determine ploidy and genomic constituents in unisexual individuals (Ramsden et al., 2006;
Bogart et al., 2007). Although we employed all of these methods to confirm ploidy in individual salamanders, the analyses of chromosome and microsatellite data from tissue collected from individuals in the present study will be the subject of future investigations that use these new techniques.

\section{Site Designation}

Because we were interested in knowing the sexual and unisexual associations in breeding populations, we tried to analyze the individuals that were expected to breed in the same population. In most cases, salamanders could be assigned to distinct breeding ponds because they were collected as adults or larvae in a pond or as newly transformed juveniles at the edge of a pond. Sometimes, however, individuals were collected on roads or in upland wooded areas and could not be assigned to any particular breeding pond. Additionally, salamanders were found at different locations entering or leaving extensive swamps that may be partitioned into subpopulations. These factors required the use of site designations based on the assumption that individuals from the same site shared a potentially common breeding area. A site may or may not be equated with a breeding population. In some instances, we combined individuals into a single site even though they were collected from extensive, yet ecologically similar, and contiguous habitat. Some other collections that were close geographically were treated as separate sites if they were distinctly separated by habitat and, in some instances, elevation. Klemens (1993) found that topography plays an important role in determining wetland type, which in turn influences the distribution of the sexual species. This was most apparent in areas of close contact where breeding ponds of the two sexual species were separated by as little as 100 meters. We grouped our sites into drainage basins because Klemens $(1978,1993)$ demonstrated that there were significant differences in the herpetofauna of New England's drainage basins, as a result of post-Pleistocene dispersion of amphibians into the interior of New England.

We numbered the sites starting with 107 as a continuation of the 106 sites sampled in our 
TABLE 3

Allele frequencies of diploid and polyploid salamanders for each of the genomotypes in appendix 2

\begin{tabular}{|c|c|c|c|c|c|c|c|c|c|}
\hline \multirow[b]{2}{*}{ Locus $^{\mathrm{a}}$} & \multicolumn{8}{|c|}{ A. laterale (LL), A. jeffersonianum (JJ), diploid, triploid, and tetraploid unisexual genomotypes ${ }^{\mathrm{b}}$} & \multirow[b]{2}{*}{ Mobility $^{\mathrm{c}}$} \\
\hline & LL & $\mathrm{JJ}$ & $\mathrm{LJ}$ & LLJ & LJJ & LLLJ & LJJJ & LLJJ & \\
\hline Aat-1 & $(308)^{\mathrm{d}}$ & $(463)$ & (86) & $(227)$ & $(244)$ & (26) & (20) & (1) & \\
\hline A & 0.003 & - & - & - & - & - & - & - & +110 \\
\hline $\mathrm{B}$ & 0.997 & - & 0.500 & 0.659 & 0.335 & 0.750 & 0.250 & 0.500 & +100 \\
\hline $\mathrm{D}$ & - & 1.000 & 0.500 & 0.341 & 0.665 & 0.250 & 0.750 & 0.500 & +79 \\
\hline Aat-2 & $(306)$ & $(462)$ & (86) & $(223)$ & $(242)$ & (25) & (20) & (1) & \\
\hline A & - & 0.995 & 0.500 & 0.335 & 0.658 & 0.250 & 0.750 & - & -180 \\
\hline B & 0.995 & 0.003 & 0.500 & 0.665 & 0.324 & 0.750 & 0.250 & 1.000 & -100 \\
\hline $\mathrm{C}$ & 0.005 & 0.002 & - & - & - & - & - & - & -50 \\
\hline Idh-1 & $(307)$ & (463) & (86) & (218) & (241) & (26) & (20) & (1) & \\
\hline Q & 0.003 & 0.002 & 0.006 & - & 0.004 & - & - & - & +160 \\
\hline A & - & 0.998 & 0.494 & 0.324 & 0.650 & 0.240 & 0.812 & 0.500 & +142 \\
\hline B & 0.995 & - & 0.500 & 0.676 & 0.346 & 0.760 & 0.188 & 0.500 & +100 \\
\hline $\mathrm{C}$ & 0.002 & - & - & - & - & - & - & - & +50 \\
\hline Ldh-1 & (302) & (462) & (85) & (220) & (243) & (26) & (20) & (1) & \\
\hline A & 0.003 & 0.002 & - & 0.003 & - & - & - & - & +115 \\
\hline B & 0.886 & - & 0.506 & 0.629 & 0.348 & 0.750 & 0.300 & 0.500 & +100 \\
\hline $\mathrm{C}$ & 0.002 & 0.998 & 0.494 & 0.327 & 0.652 & 0.250 & 0.700 & 0.500 & +88 \\
\hline D & 0.109 & - & - & 0.041 & - & - & - & - & +78 \\
\hline Ldh-2 & $(306)$ & $(463)$ & (83) & $(222)$ & $(243)$ & (26) & (20) & (1) & \\
\hline Q & - & 0.003 & - & 0.002 & - & - & - & - & +160 \\
\hline A & 0.230 & - & 0.060 & 0.123 & 0.027 & 0.038 & 0.012 & - & +130 \\
\hline B & 0.767 & 0.997 & 0.874 & 0.874 & 0.971 & 0.952 & 0.988 & 1.000 & +100 \\
\hline $\mathrm{C}$ & 0.003 & - & 0.066 & 0.002 & 0.001 & 0.010 & - & - & +55 \\
\hline Mdh-1 & $(306)$ & $(462)$ & (92) & $(227)$ & $(244)$ & (26) & (20) & (1) & \\
\hline A & - & 0.002 & - & - & 0.001 & - & - & - & +200 \\
\hline B & - & 0.948 & 0.500 & 0.352 & 0.664 & 0.250 & 0.750 & 0.500 & +176 \\
\hline $\mathrm{C}$ & 0.005 & - & - & - & 0.008 & - & - & - & +135 \\
\hline $\mathrm{D}$ & 0.995 & 0.050 & 0.500 & 0.648 & 0.326 & 0.750 & 0.250 & 0.500 & +100 \\
\hline Mpi & (253) & (316) & (58) & $(180)$ & (186) & (16) & (9) & (1) & \\
\hline A & - & 0.003 & 0.086 & 0.007 & 0.027 & - & - & - & +140 \\
\hline B & - & 0.967 & 0.509 & 0.320 & 0.640 & 0.344 & 0.778 & - & +120 \\
\hline $\mathrm{C}$ & 0.990 & 0.028 & 0.388 & 0.672 & 0.330 & 0.656 & 0.222 & 1.000 & +100 \\
\hline $\mathrm{D}$ & 0.010 & 0.002 & 0.017 & - & 0.004 & - & - & - & +80 \\
\hline Pgi & $(162)$ & $(274)$ & (42) & (153) & $(235)$ & (20) & (16) & (1) & \\
\hline Q & - & 0.005 & - & 0.002 & - & - & - & - & +380 \\
\hline A & 0.037 & 0.663 & 0.381 & 0.285 & 0.567 & 0.225 & 0.531 & 0.500 & +325 \\
\hline B & 0.009 & 0.316 & 0.083 & 0.102 & 0.074 & 0.150 & 0.094 & - & +115 \\
\hline $\mathrm{C}$ & 0.954 & 0.016 & 0.536 & 0.610 & 0.359 & 0.625 & 0.375 & 0.500 & +100 \\
\hline Pgm-1 & (306) & $(453)$ & (85) & (216) & $(235)$ & (25) & (19) & (1) & \\
\hline A & 0.015 & 0.001 & - & 0.002 & - & - & - & - & +115 \\
\hline B & 0.974 & 0.646 & 0.794 & 0.821 & 0.675 & 0.940 & 0.647 & 1.000 & +100 \\
\hline $\mathrm{C}$ & 0.011 & 0.346 & 0.206 & 0.176 & 0.325 & 0.060 & 0.353 & - & +97 \\
\hline D & - & 0.007 & - & 0.002 & - & - & - & - & +82 \\
\hline
\end{tabular}


TABLE 3

(Continued)

\begin{tabular}{cccccccccc}
\hline \hline & \multicolumn{8}{c}{ A. laterale $(\mathrm{LL})$, A. jeffersonianum $(\mathrm{JJ})$, diploid, triploid, and tetraploid unisexual genomotypes } & \\
\cline { 2 - 7 } Locus $^{\mathrm{b}}$ & LL & JJ & LJ & LLJ & LJJ & LLLJ & LJJJ & LLJJ & Mobility $^{\mathrm{c}}$ \\
\hline Pgm-2 & $(308)$ & $(460)$ & $(86)$ & $(219)$ & $(243)$ & $(26)$ & $(20)$ & $(1)$ & \\
A & 0.018 & - & - & - & - & - & - & - & +120 \\
B & 0.980 & 1.000 & 1.000 & 1.000 & 0.999 & 1.000 & 1.000 & 1.000 & +100 \\
C & 0.002 & - & - & - & 0.001 & - & - & - & +75 \\
Sod-1 & $(313)$ & $(463)$ & $(86)$ & $(226)$ & $(244)$ & $(24)$ & $(20)$ & $(1)$ & \\
B & 1.000 & - & 0.483 & 0.671 & 0.333 & 0.769 & 0.238 & 0.500 & +100 \\
D & - & 1.000 & 0.517 & 0.329 & 0.667 & 0.231 & 0.762 & 0.500 & +37 \\
\hline
\end{tabular}

${ }^{a}$ Isozyme loci used to identify the sexual and unisexual individuals. The allozymes or alleles that were observed are included under each locus.

${ }^{\mathrm{b}}$ Genomotypes are the nuclear genomes inferred by the alleles that are diagnostic for Ambystoma laterale (L) and A. jeffersonianum $(\mathrm{J})$ found in each individual.

${ }^{\mathrm{c}}$ Mobilities are the relative mobilities on the gel compared with the most common allele found in $A$. laterale that is assigned a mobility of 100 . Positive mobilities $(+)$ indicate migration from the origin toward the anode and negative mobilities $(-)$ indicate migration toward the cathode.

${ }^{\mathrm{d}}$ Sample sizes in parentheses (n) are the number of individuals of each genomotype that were examined and scored for each locus (from appendix 2).

earlier study (Bogart and Klemens, 1997). Eight sites that were re-sampled from our earlier study were not given new site numbers in the present study.

\section{Nomenclature}

Identifying and naming unisexual individuals is a formidable challenge for taxonomists and conservationists. The unisexuals do have a hybrid nuclear genomic constitution, but they are not hybrids that have resulted from the crossing of $A$. laterale and $A$. jeffersonianum or any combination of the four species whose genomes might be found in a unisexual. Genomes are gained and lost by kleptogenesis, which is driven entirely by male sperm donors. In this flexible genetic system, a single unisexual female can produce offspring that have differing genotypes and genomes (Bogart et al., 1987, 2007) and, because of intergenomic interaction (Bi and Bogart, 2006; Bi et al., 2007), genomes found in offspring from a particular unisexual might evolve within that unisexual independently of male genomic contribution. Lowcock et al. (1987) suggested an informal descriptive system for unisexual Ambystoma that was used by Schultz (1969) to describe the genetic composition of hybridogenetic unisexual fish of the genus Poeciliopsis.
Letter designations for species that contributed genomes to unisexual Ambystoma have previously been used for convenience in a number of previous studies (Uzzell, 1964; Bogart et al., 1985, 1987; Bogart and Klemens, 1997): $\mathrm{J}$ for $A$. jeffersonianum, $\mathrm{L}$ for $A$. laterale, $\mathrm{T}$ for $A$. texanum, and $\mathrm{Ti}$ for $A$. tigrinum. The proposed name, for example, for a triploid unisexual that has a nuclear genome consisting of one $A$. laterale genome and two $A$. jeffersonianum genomes would be Ambystoma laterale - (2) jeffersonianum, or LJJ.

\section{Genotype and Genomotype Analysis}

The unisexuals are mostly fixed heterozygotes for a number of isozyme alleles and their mode of reproduction is kleptogenesis. Therefore, population genetic models that are based on randomly interbreeding individuals do not apply to the unisexual kleptogens. When unisexuals occur in a population, they usually outnumber individuals of the sexual species (Bogart and Klemens, 1997). If the sample of individuals obtained from a site contained unisexuals, but neither A. laterale nor $A$. jeffersonianum individuals were found, we suspected that the sample was too small to have encountered these species. In order to obtain some measure of the influence, or the 
TABLE 4

Ambystoma laterale (LL), A. jeffersonianum (JJ), and nuclear hybrid genomes found at each site

\begin{tabular}{|c|c|c|c|c|c|c|c|c|c|c|c|}
\hline \multirow[b]{3}{*}{ Site } & \multirow[b]{3}{*}{$(\mathrm{n})^{\mathrm{a}}$} & \multirow[b]{3}{*}{ Males $^{\mathrm{b}}$} & \multicolumn{8}{|c|}{ Genome $^{c}$} & \multirow[b]{3}{*}{$\% L^{d}$} \\
\hline & & & \multicolumn{3}{|c|}{$2 n$} & \multicolumn{2}{|c|}{$3 n$} & \multicolumn{3}{|c|}{$4 n$} & \\
\hline & & & $\mathrm{LL}$ & $\mathrm{JJ}$ & $\mathrm{LJ}$ & LLJ & LJJ & LLLJ & LLJJ & LJJJ & \\
\hline $2 \dagger$ & (35) & 24 & - & 35 & - & - & - & - & - & - & 00.0 \\
\hline $7 \dagger$ & (4) & 0 & - & - & - & 1 & - & 3 & - & - & 73.3 \\
\hline $18 \dagger$ & (5) & 1 & 2 & - & 1 & 1 & - & - & 1 & - & 69.2 \\
\hline $20 \dagger$ & (2) & 0 & - & - & - & - & 2 & - & - & - & 33.3 \\
\hline $28 \dagger$ & (1) & 0 & - & - & - & - & 1 & - & - & - & 33.3 \\
\hline $42 \dagger$ & (6) & 0 & 1 & - & - & 5 & - & - & - & - & 70.6 \\
\hline $60 \dagger$ & (2) & 1 & 2 & - & - & - & - & - & - & - & 100.0 \\
\hline $66 \dagger$ & (2) & 1 & 2 & - & - & - & - & - & - & - & 100.0 \\
\hline 107 & (25) & 3 & 9 & - & 4 & 7 & - & 5 & - & - & 76.1 \\
\hline 108 & (43) & $11^{*}$ & 14 & - & 7 & 17 & 2 & 3 & - & - & 72.1 \\
\hline 109 & (9) & 0 & - & - & 2 & - & 4 & - & - & 3 & 31.0 \\
\hline 110 & (31) & 6 & 19 & - & 1 & 9 & - & 2 & - & - & 85.3 \\
\hline 111 & (26) & 3 & 9 & - & - & 14 & - & 3 & - & - & 68.0 \\
\hline 112 & (5) & $1^{*}$ & - & - & 1 & 4 & - & - & - & - & 64.3 \\
\hline 113 & (43) & 4 & 6 & - & 18 & 17 & 1 & 1 & - & - & 64.2 \\
\hline 114 & (46) & $20^{*}$ & - & 44 & 1 & - & 1 & - & - & - & 2.2 \\
\hline 115 & (3) & 1 & - & 1 & - & - & 2 & - & - & - & 25.0 \\
\hline 116 & (1) & 0 & - & 1 & - & - & - & - & - & - & 00.0 \\
\hline 117 & (9) & 0 & - & - & - & - & 5 & - & - & 4 & 29.0 \\
\hline 118 & (16) & 10 & - & 16 & - & - & - & - & - & - & 00.0 \\
\hline 119 & (7) & 0 & - & 1 & - & - & 6 & - & - & - & 30.0 \\
\hline 120 & (14) & 5 & - & 14 & - & - & - & - & - & - & 00.0 \\
\hline 121 & (20) & 13 & - & 20 & - & - & - & - & - & - & 00.0 \\
\hline 122 & (7) & $1 *$ & - & - & 4 & - & 3 & - & - & - & 41.2 \\
\hline 123 & (14) & 1 & - & 1 & 11 & 1 & 1 & - & - & - & 46.7 \\
\hline 124 & (11) & 0 & - & 1 & 3 & - & 7 & - & - & - & 34.5 \\
\hline 125 & (5) & 0 & - & 2 & 2 & 1 & - & - & - & - & 36.4 \\
\hline 126 & (30) & 3 & - & 5 & 5 & - & 18 & - & - & 2 & 31.2 \\
\hline 127 & (51) & 23 & 41 & - & - & 10 & - & - & - & - & 91.1 \\
\hline 128 & (1) & 0 & - & - & - & 1 & - & - & - & - & 66.7 \\
\hline 129 & (12) & 2 & 8 & - & - & 4 & - & - & - & - & 85.7 \\
\hline 130 & (13) & 1 & - & 6 & 1 & - & 6 & - & - & - & 21.9 \\
\hline 131 & (6) & 1 & - & 1 & - & - & 5 & - & - & - & 29.4 \\
\hline 132 & (2) & 0 & 1 & - & - & 1 & - & - & - & - & 80.0 \\
\hline 133 & (7) & 1 & 3 & - & - & 4 & - & - & - & - & 77.8 \\
\hline 134 & (2) & 0 & 1 & - & 1 & - & - & - & - & - & 75.0 \\
\hline 135 & (1) & 1 & - & 1 & - & - & - & - & - & - & 00.0 \\
\hline 136 & (18) & 4 & 7 & - & - & 11 & - & - & - & - & 76.6 \\
\hline 137 & (12) & 6 & - & 9 & 2 & - & 1 & - & - & - & 12.0 \\
\hline 138 & (4) & 1 & - & 2 & 1 & - & 1 & - & - & - & 22.2 \\
\hline 139 & (1) & 0 & - & - & - & - & 1 & - & - & - & 33.3 \\
\hline 140 & (4) & 1 & - & 1 & - & - & 3 & - & - & - & 27.3 \\
\hline 141 & (3) & 0 & - & - & - & - & 3 & - & - & - & 33.3 \\
\hline 142 & (1) & 0 & - & - & - & - & 1 & - & - & - & 33.3 \\
\hline 143 & (17) & 8 & 13 & - & - & 4 & - & - & - & - & 89.5 \\
\hline 144 & (10) & 4 & 7 & - & - & 3 & - & - & - & - & 87.0 \\
\hline 145 & (19) & 2 & 4 & - & - & 15 & - & - & - & - & 73.1 \\
\hline 146 & (1) & 1 & 1 & - & - & - & - & - & - & - & 100.0 \\
\hline 147 & (7) & 6 & 7 & - & - & - & - & - & - & - & 100.0 \\
\hline 148 & (9) & $1^{*}$ & - & - & - & 8 & - & 1 & - & - & 67.8 \\
\hline
\end{tabular}


TABLE 4

(Continued)

\begin{tabular}{|c|c|c|c|c|c|c|c|c|c|c|c|}
\hline \multirow[b]{3}{*}{ Site } & \multirow[b]{3}{*}{$(\mathrm{n})^{\mathrm{a}}$} & \multirow[b]{3}{*}{ Males ${ }^{\mathrm{b}}$} & \multicolumn{8}{|c|}{ Genome $^{\mathrm{c}}$} & \multirow[b]{3}{*}{$\% \mathrm{~L}^{\mathrm{d}}$} \\
\hline & & & \multicolumn{3}{|c|}{$2 n$} & \multicolumn{2}{|c|}{$3 n$} & \multicolumn{3}{|c|}{$4 n$} & \\
\hline & & & LL & $\mathrm{JJ}$ & LJ & LLJ & LJJ & LLLJ & LLJJ & LJJJ & \\
\hline 149 & (1) & 0 & - & - & - & 1 & - & - & - & - & 66.7 \\
\hline 150 & (17) & 4 & 17 & - & - & - & - & - & - & - & 100.0 \\
\hline 151 & (9) & 4 & 7 & - & - & 2 & - & - & - & - & 90.0 \\
\hline 152 & (10) & $5^{*}$ & 8 & - & - & 2 & - & - & - & - & 90.9 \\
\hline 153 & (1) & 0 & 1 & - & - & - & - & - & - & - & 100.0 \\
\hline 154 & (13) & 8 & 13 & - & - & - & - & - & - & - & 100.0 \\
\hline 155 & (10) & 1 & 2 & - & - & 7 & - & 1 & - & - & 72.4 \\
\hline 156 & (13) & 5 & - & 6 & 3 & - & 4 & - & - & - & 23.3 \\
\hline 157 & (8) & 1 & - & 1 & - & - & 7 & - & - & - & 30.4 \\
\hline 158 & (13) & 6 & - & 7 & 1 & - & 5 & - & - & - & 19.4 \\
\hline 159 & (9) & 1 & - & 2 & 1 & - & 6 & - & - & - & 29.2 \\
\hline 160 & (4) & 0 & - & - & - & - & 4 & - & - & - & 33.3 \\
\hline 161 & (11) & 0 & - & - & - & 3 & 7 & - & - & 1 & 41.2 \\
\hline 162 & (16) & $5^{*}$ & 5 & - & - & 10 & - & 1 & - & - & 75.0 \\
\hline 163 & (12) & 2 & - & 3 & - & - & 9 & - & - & - & 27.3 \\
\hline 164 & (12) & 5 & - & 6 & - & - & 6 & - & - & - & 20.0 \\
\hline 165 & (17) & $1^{*}$ & - & - & - & - & 17 & - & - & - & 33.3 \\
\hline 166 & (15) & 2 & - & 3 & - & - & 12 & - & - & - & 28.6 \\
\hline 167 & (6) & 2 & - & 3 & - & - & 3 & - & - & - & 20.0 \\
\hline 168 & (38) & 4 & 9 & - & - & 26 & - & 3 & - & - & 90.6 \\
\hline 169 & (7) & 1 & - & 1 & 1 & - & 5 & - & - & - & 31.6 \\
\hline 170 & (33) & 25 & - & 33 & - & - & - & - & - & - & 00.0 \\
\hline 171 & (10) & 8 & - & 10 & - & - & - & - & - & - & 00.0 \\
\hline 172 & (9) & 5 & - & 9 & - & - & - & - & - & - & 00.0 \\
\hline 173 & (17) & 10 & - & 17 & - & - & - & - & - & - & 00.0 \\
\hline 174 & (3) & 0 & - & - & 1 & 2 & - & - & - & - & 62.5 \\
\hline 175 & (21) & 15 & - & 21 & - & - & - & - & - & - & 00.0 \\
\hline 176 & (1) & 0 & - & 1 & - & - & - & - & - & - & 00.0 \\
\hline 177 & (5) & 5 & - & 5 & - & - & - & - & - & - & 00.0 \\
\hline 178 & (1) & 0 & - & 1 & - & - & - & - & - & - & 00.0 \\
\hline 179 & (17) & 4 & - & 9 & - & - & 7 & - & - & 1 & 18.6 \\
\hline 180 & (25) & 7 & - & 25 & - & - & - & - & - & - & 00.0 \\
\hline 181 & (16) & 10 & - & 16 & - & - & - & - & - & - & 00.0 \\
\hline 182 & (26) & 15 & - & 26 & - & - & - & - & - & - & 00.0 \\
\hline 183 & (16) & 7 & - & 16 & - & - & - & - & - & - & 00.0 \\
\hline 184 & (2) & 2 & - & 2 & - & - & - & - & - & - & 00.0 \\
\hline 185 & (18) & 14 & - & 18 & - & - & - & - & - & - & 00.0 \\
\hline 186 & (18) & 12 & - & 18 & - & - & - & - & - & - & 00.0 \\
\hline 187 & (12) & 4 & - & 12 & - & - & - & - & - & - & 00.0 \\
\hline 188 & (10) & 10 & - & 10 & - & - & - & - & - & - & 00.0 \\
\hline 189 & (65) & 24 & 45 & - & - & 20 & - & - & - & - & 86.7 \\
\hline 190 & (4) & 4 & - & 4 & - & - & - & - & - & - & 00.0 \\
\hline 191 & (9) & 3 & - & 3 & - & - & 6 & - & - & - & 25.0 \\
\hline 192 & (4) & 1 & - & 1 & 1 & - & 1 & - & - & 1 & 27.3 \\
\hline 193 & (3) & 0 & - & - & - & - & 3 & - & - & - & 33.3 \\
\hline 194 & (9) & 1 & - & 1 & - & - & 7 & - & - & 1 & 29.6 \\
\hline 195 & (15) & 4 & - & 6 & - & - & 9 & - & - & - & 23.1 \\
\hline 196 & (3) & 1 & - & 3 & - & - & - & - & - & - & 00.0 \\
\hline 197 & (1) & 0 & - & - & - & 1 & - & - & - & - & 66.7 \\
\hline 198 & (13) & 3 & 4 & - & - & 2 & 4 & 3 & - & - & 67.6 \\
\hline
\end{tabular}


TABLE 4

(Continued)

\begin{tabular}{|c|c|c|c|c|c|c|c|c|c|c|c|}
\hline \multirow[b]{3}{*}{ Site } & \multirow[b]{3}{*}{$(\mathrm{n})^{\mathrm{a}}$} & \multirow[b]{3}{*}{ Males $^{\mathrm{b}}$} & \multicolumn{8}{|c|}{ Genome $^{\mathrm{c}}$} & \multirow[b]{3}{*}{$\% \mathrm{~L}^{\mathrm{d}}$} \\
\hline & & & \multicolumn{3}{|c|}{$2 n$} & \multicolumn{2}{|c|}{$3 n$} & \multicolumn{3}{|c|}{$4 n$} & \\
\hline & & & LL & $\mathrm{JJ}$ & $\mathrm{LJ}$ & LLJ & LJJ & LLLJ & LLJJ & LJJJ & \\
\hline 199 & (1) & 0 & 1 & - & - & - & - & - & - & - & 100.0 \\
\hline 200 & (9) & 0 & - & - & - & - & 9 & - & - & - & 33.3 \\
\hline 201 & (7) & 0 & - & - & 7 & - & - & - & - & - & 50.0 \\
\hline 202 & (3) & 0 & - & - & - & - & 3 & - & - & - & 33.3 \\
\hline 203 & (17) & 1 & - & 1 & - & - & 11 & - & - & 5 & 31.4 \\
\hline 204 & (20) & 14 & 20 & - & - & - & - & - & - & - & 100.0 \\
\hline 205 & (1) & 0 & - & 1 & - & - & - & - & - & - & 00.0 \\
\hline 206 & (2) & 1 & 2 & - & - & - & - & - & - & - & 100.0 \\
\hline 207 & (12) & 0 & - & - & - & - & 10 & - & - & 2 & 31.6 \\
\hline 208 & (14) & 0 & - & - & - & - & 14 & - & - & - & 33.3 \\
\hline 209 & (1) & 0 & - & - & - & 1 & - & - & - & - & 66.7 \\
\hline 210 & (5) & 3 & 5 & - & - & - & - & - & - & - & 100.0 \\
\hline 211 & (14) & 9 & 10 & - & 2 & 2 & - & - & - & - & 86.7 \\
\hline 212 & (2) & 1 & - & 2 & - & - & - & - & - & - & 00.0 \\
\hline 213 & (1) & 0 & - & - & - & - & 1 & - & - & - & 33.3 \\
\hline 214 & (4) & 0 & 1 & - & 2 & 1 & - & - & - & - & 55.6 \\
\hline 215 & (12) & 1 & 1 & - & 2 & 9 & - & - & - & - & 66.7 \\
\hline 216 & (1) & 0 & - & - & - & 1 & - & - & - & - & 66.7 \\
\hline Total & (1377) & 453 & 308 & 464 & 86 & 228 & 244 & 26 & 1 & 20 & \\
\hline \multirow[t]{4}{*}{ Percentages } & & 32.90 & 22.37 & 33.70 & 6.24 & 16.56 & 17.72 & 1.89 & 0.07 & 1.45 & \\
\hline & \multicolumn{5}{|c|}{ Percent unisexuals: 43.94} & \multicolumn{6}{|c|}{ Percent unisexual triploids: 78.02} \\
\hline & \multicolumn{5}{|c|}{ Percent "unsexual males": 1.32} & \multicolumn{6}{|c|}{ Percent unisexual tetraploids: 7.77} \\
\hline & \multicolumn{5}{|c|}{ Percent unisexual diploids: 14.21} & & & & & & \\
\hline
\end{tabular}

${ }^{a}$ Sites and individual specimens are provided in appendix 1 and appendix 2 .

$\mathrm{b} *$ indicates the finding of a rare "unisexual male" at this site.

${ }^{c}$ Electrophoretically identified genotypes of diploid, triploid, and tetraploid salamanders: Ambystoma laterale genome (L) and $A$. jeffersonianum genome (J).

${ }^{\mathrm{d}}$ Percentage of Ambystoma laterale genomes $(\% \mathrm{~L})$ is calculated from all individuals collected at each site.

$\dagger$ New individuals collected from the same sites previously examined by Bogart and Klemens (1997).

presence of the sexual species, we calculated the genomic percentage of $A$. laterale in each site to obtain values ranging from $0 \%$ (all $A$. jeffersonianum) to $100 \%$ (all $A$. laterale). Under this scheme, a triploid LLJ would be $66.7 \%$ (A. laterale) and triploid LJJ would be $33.3 \%$ (A. laterale). If the average percentage of all the individuals from a site was below 50 then $A$. jeffersonianum is assumed to be the sperm donor in that population even though the sample of salamanders may not have included $A$. jeffersonianum. We use the term genomotype (Lowcock, 1994) to describe the template genomic contributions of $A$. laterale (LL) and $A$. jeffersonianum (JJ) in diploid and polyploid unisexual individuals while recognizing that the combination of genomes in unisexuals may be slightly restructured by intergenomic recombinations and translocations (Bi and Bogart, 2006; Bi et al., 2007).

\section{RESULTS}

\section{ELECTROPHORESIS}

As in our previous study (Bogart and Klemens, 1997), A. laterale could be easily distinguished from $A$. jeffersonianum individuals based on the presence of alternate electrophoretic alleles that were mostly homozygous with a gene frequency $(p)>0.90$ in both species. The genotypes for $A$. laterale (appendix 2-1) and $A$. jeffersonianum (appendix 2-2) individuals and their allele frequencies for each locus (table 3 ) demonstrate considerable intraspecific homozygosity for most of 
the 11 loci examined, but Sod-1 was the only locus that was alternately fixed $(p=1.00)$ for Sod- $1^{100}$ in $A$. laterale and Sod $-1^{37}$ in $A$. jeffersonianum. Gene frequencies at each of the 11 loci were calculated for the sexual genotypes and unisexual genomotypes from all sites (table 3) based on the data for all individuals (appendix 2). The common allele $(p)>0.95$ for $A$. jeffersonianum was not found in any $A$. laterale specimen for Aat- $1^{79}$, Aat$2^{-180}$, Idh- $1^{142}$, Mdh- $1^{176}$, and $\mathrm{Mpi}^{120}$. Based on these diagnostic isozyme alleles and ploidy determination (below), the unisexual specimens were identified as A. laterale - jeffersonianum (LJ) (appendix 2-3); A. (2) laterale jeffersonianum (LLJ) (appendix 2-4); A. laterale - (2) jeffersonianum (LJJ) (appendix 2-5); A. (3) laterale - jeffersonianum (LLLJ) (appendix 2-6); A. laterale - (3) jeffersonianum (LJJJ) (appendix 2-7); and A. (2) laterale - (2) jeffersonianum (LLJJ) (appendix 2-8). The sites (appendix 1) where the sexual and unisexual individuals were found are included for each individual in appendix 2. A summation of the sexual and unisexual genomotypes at each site is provided in table 4 . We found more unisexual individuals $(44 \%)$ than either of the sexual species (23\% LL and $34 \% \mathrm{JJ})$ and most unisexuals were triploid. Tetraploids $(3 \%)$ and unisexual males $(1 \%)$ were rare and were found with unisexual diploid and/or triploid females in scattered populations.

\section{New and Rare Alleles}

Sod-1 was fixed for one allele in A. laterale and one in $A$. jeffersonianum but we found additional alleles at the other loci in a few individuals of the two species and the unisexuals. Some rare alleles $(p)<0.05$ were also found in our earlier study in individual $A$. laterale (Aat- ${ }^{110}$, Aat-2 ${ }^{-50}$, Ldh- $1^{88}$, Ldh- $2^{55}$, Mdh-1 ${ }^{135}, \mathrm{Pgi}^{325}, \mathrm{Pgi}^{115}, \mathrm{Pgm}-1^{97}, \mathrm{Pgm}-2^{120}$, Pgm- $2^{75}$ ) and $A$. jeffersonianum (Aat $1^{100}$, Aat- $2^{-100}$, Aat-2 $2^{-50}$, Idh-1 ${ }^{100}$, Ldh-1 ${ }^{115}$, Mdh-1 ${ }^{100}, \mathrm{Mpi}^{140}, \mathrm{Mpi}^{100}, \mathrm{Pgi}^{100}, \mathrm{Pgm}^{10}{ }^{82}$ ). In the present study we found most of these same rare alleles and we also found nine new alleles that were not encountered by Bogart and Klemens (1997). A new Ldh-1 $1^{78}$ allele (D) with a frequency $(p)$ of 0.109 in A. laterale (table 3 ) is not a rare allele. This allele was found in both $A$. laterale and LLJ unisexual specimens in northern Pennsylvania where it was found in a homozygous condition in $A$. laterale and unisexual LLJ individuals (site 189). Unisexual LLJ from site 148 in western New York were also homozygous for $\mathrm{Ldh}-1^{78}$. Ambystoma laterale was not collected at that site but is presumed to be the sperm donor $(67.8 \% \mathrm{~L}$, table 4$)$. The other eight new alleles that were found were rare $(p)<0.05$. Aat- $1^{86}$ was found only as a heterozygous allele in one LLJ (site 145) in western New York. Two new Idh-1 alleles were found (Idh- ${ }^{160}$ and Idh$\left.1^{50}\right)$. The Idh- $1^{160}$ allele migrated more anodally on the gel than the Idh- ${ }^{142}$ (A) allele and is designated $\mathrm{Q}$ in appendix 2 and table 3. That allele was found in a heterozygote condition in $A$. laterale (sites 113 and 189), A. jeffersonianum (sites 118 and 204), and in unisexual LJ and LJJ individuals (site 130). The Idh- $1^{50}$ allele was found in only one $A$. laterale QC heterozygous individual (site 113). A new Ldh-2 160 , also a "Q" allele was found in $A$. jeffersonianum heterozygotes (sites 2 and 120 ) in western New York. This allele was not found in the two individuals from site 2 that were sampled by Bogart and Klemens (1997). Both site 2 and site 120 are in Tompkins County. One LLJ heterozygote from site 208 in eastern New York also possessed the Ldh$2^{160} \mathrm{Q}$ allele. A new Mdh- $1^{200}$ allele was found in $A$. jeffersonianum and an LJJ unisexual from site 158 in Morris County, New Jersey. A new $\mathrm{Mpi}^{80}$ allele was found in $A$. laterale from distant localities (sites 108 and 150), $A$. jeffersonianum (site 138), LJ (site 112), and LJJ (site 126). A new $\mathrm{Pgi}^{380} \mathrm{Q}$ allele was found in scattered populations that included $A$. jeffersonianum (sites 118, 170, 185) and one LLJ unisexual (site 155). A new Pgm- $1^{115}$ allele was found in $A$. laterale from western (site 127) and northern New York (sites 150 and 151) as well as in A. jeffersonianum from southeastern New York (site 121) and in one unisexual LLJ individual from site 148 in western New York.

\section{Homozygosity and Reversed Genotypes IN UNISEXUALS}

The majority of the unisexuals were heterozygous and were identified by the observed 
TABLE 5

Unisexual individuals that demonstrated an unexpected homozygous condition for alleles at loci diagnostic for $A$. laterale and $A$. jeffersonianum

Sample sizes in parentheses (n) are the number of individuals of each genomotype that were examined and scored for each locus (from appendix 2) (see footnotes in table 3).

\begin{tabular}{|c|c|c|c|c|c|c|c|c|c|}
\hline \multirow[b]{2}{*}{ Locus } & \multirow[b]{2}{*}{ Mobility } & \multicolumn{6}{|c|}{ Diploid, triploid, and tetraploid unisexual genomotypes } & \multirow[b]{2}{*}{ Total } & \multirow[b]{2}{*}{$\%$} \\
\hline & & $\mathrm{LJ}$ & LLJ & LJJ & LLLJ & LJJJ & LLJJ & & \\
\hline Aat-2 (n) & & $(86)$ & $(226)$ & $(242)$ & $(25)$ & (19) & (1) & $(599)$ & \\
\hline $\mathrm{B}$ & -100 & & & & & & 1 & (1) & 0.17 \\
\hline Idh-1 (n) & & $(86)$ & $(218)$ & $(241)$ & (26) & $(20)$ & (1) & $(592)$ & \\
\hline A & +142 & 2 & 3 & 12 & & 5 & & (22) & 3.72 \\
\hline B & +100 & 1 & 16 & & 2 & & & (19) & 3.21 \\
\hline Ldh-1 (n) & & (85) & (220) & (243) & (26) & (20) & (1) & (595) & \\
\hline B & +100 & 2 & 4 & & 1 & & & (7) & 1.18 \\
\hline $\mathrm{C}$ & +88 & 1 & 1 & 1 & & & & (3) & 0.50 \\
\hline $\mathrm{D}$ & +78 & & 9 & & & & & (9) & 1.51 \\
\hline Mdh-1 (n) & & (84) & $(227)$ & (244) & (26) & (20) & (1) & (602) & \\
\hline $\mathrm{D}$ & +100 & & 2 & & & & & (2) & 0.33 \\
\hline Mpi (n) & & $(58)$ & (180) & (186) & (16) & (9) & (1) & $(450)$ & \\
\hline A & +140 & & 1 & & & & & (1) & 0.22 \\
\hline B & +120 & 1 & 1 & 1 & 2 & 1 & & (6) & 1.33 \\
\hline $\mathrm{C}$ & +100 & & 11 & & & & 1 & (12) & 2.67 \\
\hline Sod-1 (n) & & (86) & (226) & (244) & (26) & (20) & (1) & $(603)$ & \\
\hline B & +100 & & 3 & & 2 & & & (5) & 0.83 \\
\hline $\mathrm{D}$ & +37 & 3 & & & & 1 & & (4) & 0.66 \\
\hline
\end{tabular}

diagnostic alleles at several loci (above). There were, however, a few unisexual individuals that were homozygous for $A$. laterale or $A$. jeffersonianum alleles at some loci. In addition, a few polyploid unisexuals had a genotype that, based on observed electrophoretic dosage, included some reversed patterns. In our earlier investigation (Bogart and Klemens, 1997), we also found homozygous alleles and reversed dosage patterns for some individuals at some loci. Of the six diagnostic loci where homozygous unisexuals were observed, 6.93\% of the unisexuals were homozygous for Idh-1 alleles. There were fewer homozygous unisexual individuals at the other loci. The number of unisexual individuals that were homozygous for alleles at one or more of the six diagnostic loci is included in table 5. Again, more unisexual polyploid individuals had a reversed electrophoretic pattern for Idh-1 diagnostic alleles $(4.8 \%)$, but the frequency of reversals found for any diagnostic allele at the seven loci involved very few individuals (table 6).

\section{PloIdy}

Data for blood cell measurements for individuals are included in appendix 2 and are summarized in table 7 and fig. 1. Although the ranges for the erythrocyte area measurements were large in all of the genomic classes, a T-test revealed a significant difference $(\mathrm{p}<0.01)$ between the ploidy classes when they were grouped as diploid, triploid, or tetraploid. We found a significant difference between measurements of $A$. laterale and A. jeffersonianum but no significant difference between the measurements of LJJ triploids and LLLJ tetraploids. These data show that A. jeffersonianum has larger blood cells than do $A$. laterale and raise the possibility that measurements of some triploid or tetraploid individuals might not accurately distinguish 
TABLE 6

Unisexual individuals that demonstrated a reversed condition for diagnostic alleles

For example, the expected genotype for an LLJ individual for Aat-1 would be Aat-1 100/100/79 or BBD and a reversed genotype at that locus would be Aat- $1^{100 / 79 / 79}$ or BDD which would be the expected genotype for LJJ. A reversal in a tetraploid LLLJ could be

Aat- $1^{100 / 100 / 79 / 79}$ or Aat- $1^{100 / 79 / 79 / 79}$. Sample sizes in parentheses (n) are the number of individuals of each genomotype that were examined and scored for each locus

(from appendix 2) (see footnotes in table 3).

\begin{tabular}{lcccccccc}
\hline \hline & & \multicolumn{2}{c}{ Diploid, triploid, and tetraploid unisexual genomotypes } & & \\
\cline { 3 - 6 } Locus & Mobility & LLJ & LJJ & LLLJ & LJJ & LLJJ & Total & $\%$ \\
\hline Aat-1 (n) & & $(227)$ & $(244)$ & $(26)$ & $(20)$ & $(1)$ & $(518)$ & \\
B & +100 & & 1 & & 0 & 0 & & 0.19 \\
D & +79 & 5 & & 0 & & 0 & 5 & 0.96 \\
Aat-2 (n) & & $(223)$ & $(242)$ & $(25)$ & $(20)$ & $(1)$ & $(505)$ & \\
A & -180 & 1 & & 0 & & 0 & 1 & 0.20 \\
B & -100 & & 6 & & 0 & 0 & 6 & 1.19 \\
Idh-1 (n) & & $(218)$ & $(241)$ & $(26)$ & $(20)$ & $(1)$ & $(499)$ & \\
A & +142 & 4 & & 1 & & 0 & 5 & 1.00 \\
B & +100 & & 22 & & 0 & 0 & 22 & 4.41 \\
Ldh-1 (n) & & $(220)$ & $(243)$ & $(26)$ & $(20)$ & $(1)$ & $(504)$ & \\
B & +100 & & 12 & & 2 & 0 & 14 & 2.58 \\
C & +88 & 7 & & 1 & & 0 & 8 & 1.39 \\
Mdh-1 (n) & & $(227)$ & $(244)$ & $(26)$ & $(20)$ & $(1)$ & $(512)$ & \\
B & +176 & 15 & & 0 & & 0 & 15 & 2.93 \\
D & +100 & & 1 & & 0 & 0 & 1 & 0.20 \\
Mpi (n) & & $(180)$ & $(186)$ & $(16)$ & $(9)$ & $(1)$ & $(387)$ & \\
B & +120 & 2 & & 0 & & 0 & 2 & 0.52 \\
C & +100 & & 7 & & 0 & 0 & 7 & 1.81 \\
Sod-1 (n) & & $(226)$ & $(235)$ & $(26)$ & $(20)$ & $(1)$ & $(511)$ & 0 \\
B & +100 & 0 & & 0 & & 0 & 0 & 0.00 \\
D & +37 & & 0 & & 0 & 0 & 0 & 0.00 \\
\hline
\end{tabular}

these ploidy classes. We have more confidence in the ploidy determinations based on flow cytometric data, but those data were not quantified as absolute picograms of DNA. Blood cells from individuals were compared with a diploid $A$. jeffersonianum standard diploid peak (Ramsden et al., 2006) to determine ploidy. Ploidy determinations for such individuals are signified by FCM in appendix 2 .

\section{Connecticut and Massachusetts}

Unisexuals were found in 14 of the 19 sites sampled in Connecticut and Massachusetts in the present study. We found one site (196) in Hartford County, Connecticut, that only had
A. jeffersonianum $(\mathrm{n}=3)$, but the sample size is too low to confirm a pure $A$. jeffersonianum population. We found only one $A$. laterale at another Hartford County site (199). Windham County sites $(60: \mathrm{n}=2 ; 204: \mathrm{n}=20)$ in Connecticut as well as a Bristol and Plymouth County site (66: $\mathrm{n}=2$ ) in Massachusetts are probably pure $A$. laterale sites. Only $A$. laterale was found in our earlier study (Bogart and Klemens, 1997) at site 60 ( $\mathrm{n}=$ $17)$ and site $66(n=4)$. In our present study, only $A$. laterale and LLJ individuals were collected in Massachusetts. Many more sites in these states were sampled by Bogart and Klemens (1997), and the distribution provided in figure 6 includes our earlier findings. Both $A$. laterale as well as $A$. jeffersonianum and 


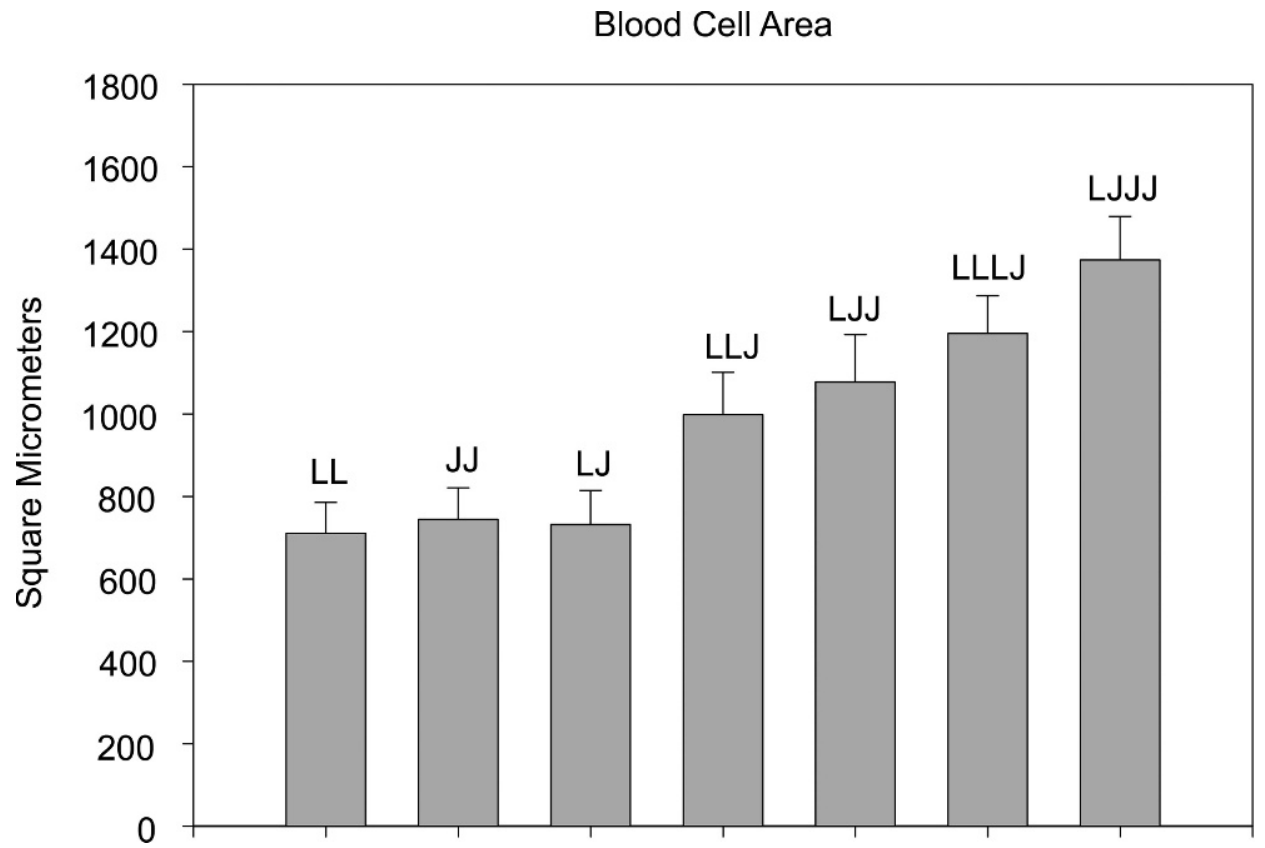

Fig. 1. Graph of mean ( $\pm \mathrm{SD}$ ) erythrocyte area for diploid Ambystoma jeffersonianum (JJ), diploid $A$. laterale (LL), diploid unisexuals (LJ), triploid unisexuals (LJJ, LLJ), and tetraploid unisexuals (LLLJ, LJJJ) from the data in table 7.

\section{TABLE 7}

Summation statistics for blood cell measurements for A. laterale (LL), A. jeffersonianum (JJ), and unisexual individuals prior to the use of flow cytometric methods (FCM) for ploidy determination Average erythrocyte values for the individuals are in appendix 2.

\begin{tabular}{|c|c|c|c|}
\hline \multicolumn{4}{|c|}{ Erythrocyte Area Measurements $\left(\mu \mathrm{m}^{2}\right)$} \\
\hline Genome $^{\mathrm{a}}$ & (n) & Mean $\pm \mathrm{SD}$ & Range \\
\hline \multicolumn{4}{|l|}{ Diploids } \\
\hline LL & (205) & $710.80 \pm 74.89$ & $515-949$ \\
\hline $\mathrm{JJ}$ & (392) & $744.54 \pm 76.26$ & $387-1063$ \\
\hline LJ & (69) & $732.16 \pm 82.85$ & $651-978$ \\
\hline \multicolumn{4}{|l|}{ Triploids } \\
\hline LLJ & (177) & $998.67 \pm 102.73$ & $670-1252$ \\
\hline LJJ & $(162)$ & $1077.89 \pm 114.75$ & $731-1503$ \\
\hline \multicolumn{4}{|c|}{ Tetraploids } \\
\hline LLLJ & (14) & $1195.28 \pm 91.94$ & $1016-1317$ \\
\hline LJJJ & (7) & $1374.00 \pm 105.26$ & $1203-1536$ \\
\hline
\end{tabular}

${ }^{\text {a }}$ Genomes include haploid complements of Ambystoma laterale (L) and A. jeffersonianum (J). their unisexual associates are widespread in these states.

\section{New Jersey}

Ambystoma laterale, A. jeffersonianum, and unisexuals have previously been reported to occur in New Jersey (Uzzell, 1964; Anderson and Giacosie, 1967). Unisexuals were found in 15 of the 17 New Jersey sites that we sampled (appendix A). The only site where unisexuals were not collected was an $A$. laterale site in Morris County (site 154: $\mathrm{n}=13$ ) in northern New Jersey. Even though more A. jeffersonianum individuals $(\mathrm{n}=31)$ were collected from more sites in New Jersey than were those of $A$. laterale $(\mathrm{n}=28)$ (table 4$)$, no site was found for $A$. jeffersonianum that did not also have unisexual individuals (fig. 4).

\section{NEW York}

Unisexuals were found in most (43 of 57) New York sites (appendix 1) (fig. 5). The northern New York sites in St. Lawrence County (sites 149 to 152 ) were dominated by 

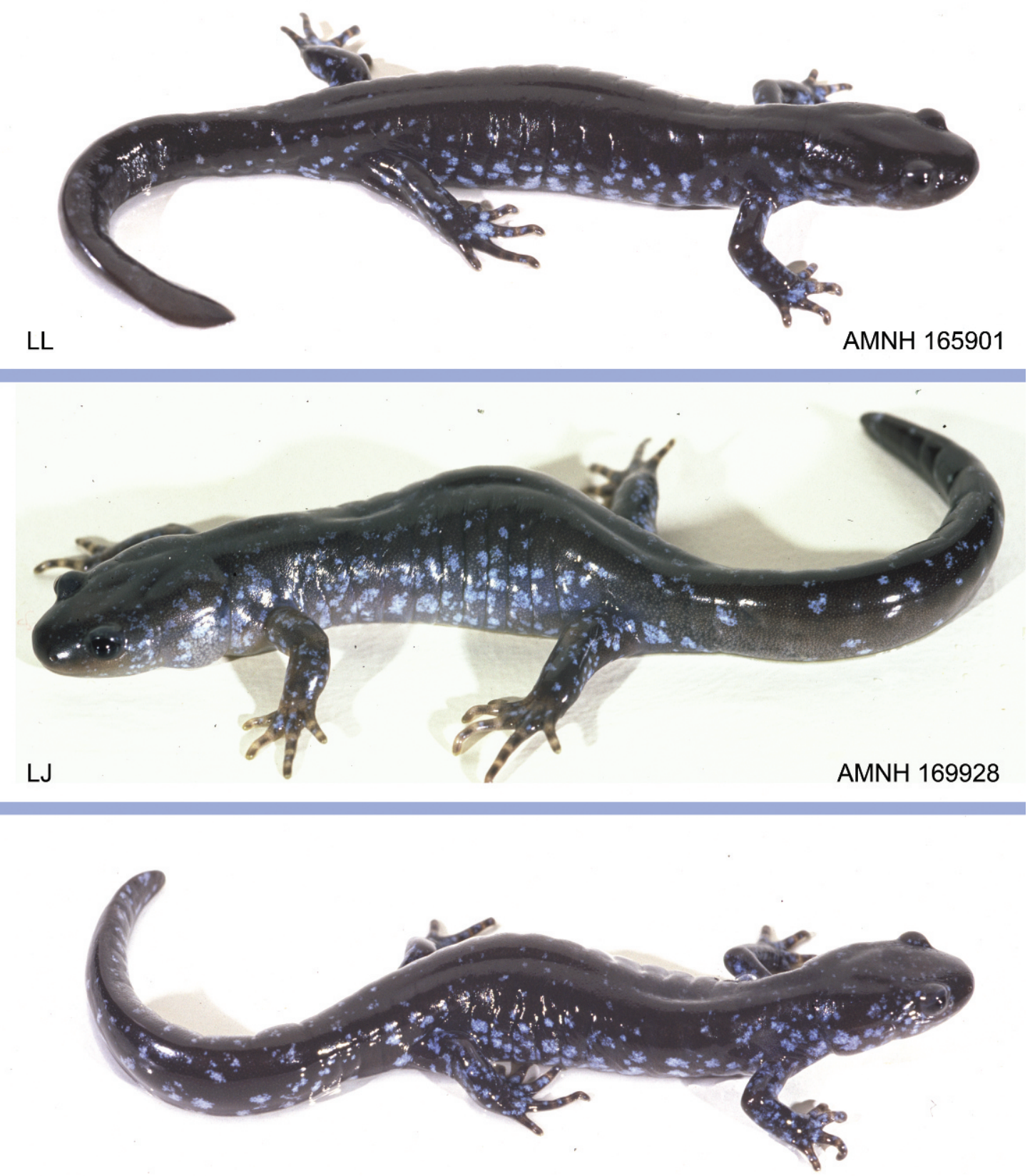

LLJ

AMNH 166022

Fig. 2. Ambystoma laterale and unisexuals associated with A. laterale found in Pennsylvania. Ambystoma laterale (AMNH 165901 from site 189) (top), diploid unisexual LJ (AMNH 169928 from site 174) (middle), and triploid unisexual LLJ (AMNH 166022 from site 189) (bottom). 

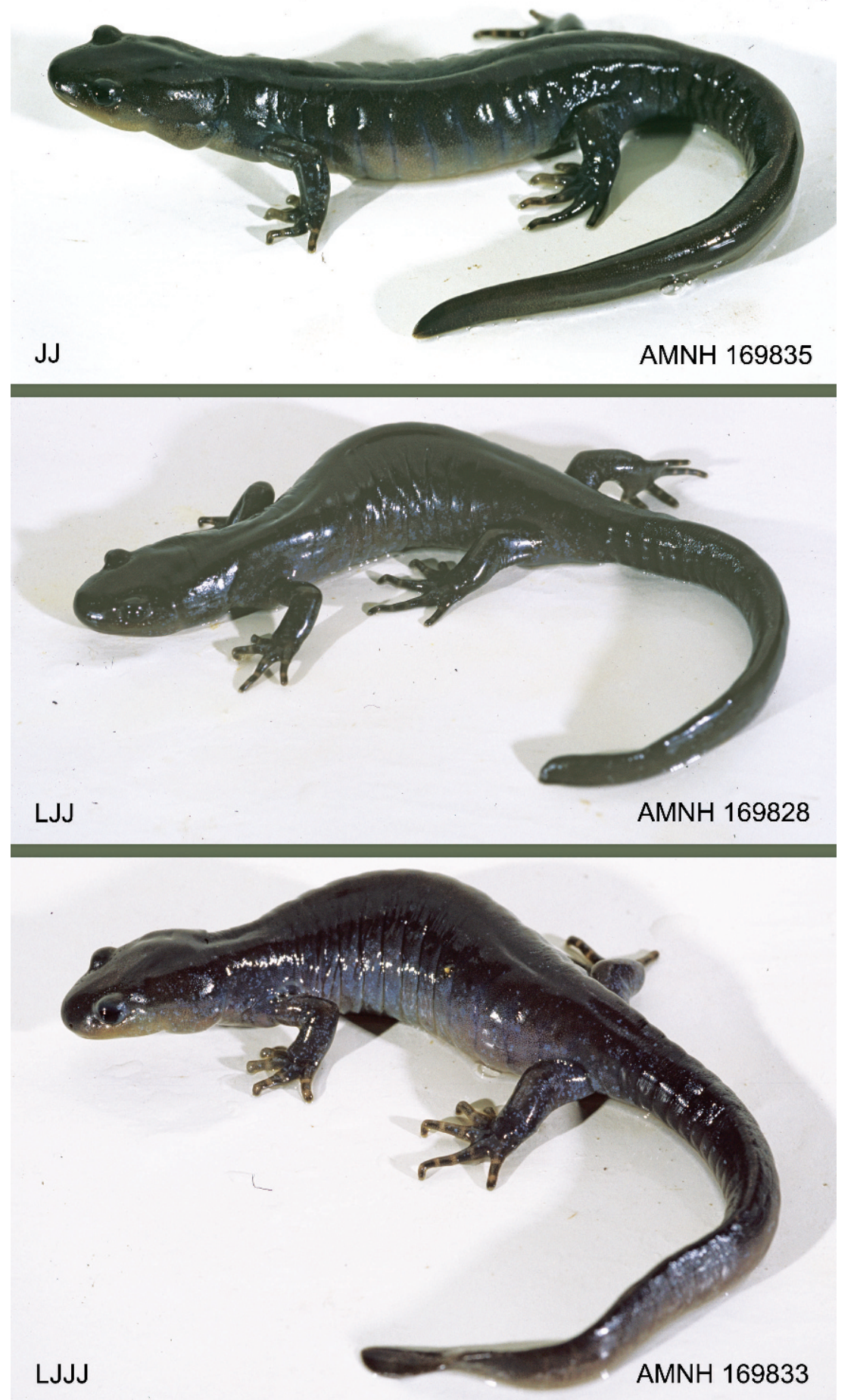

Fig. 3. Ambystoma jeffersonianum and unisexuals associated with A. jeffersonianum found in Pennsylvania. Ambystoma jeffersonianum (AMNH 169835) (top), triploid unisexual (LJJ) (AMNH 169828) (middle), and tetraploid LJJJ unisexual (AMNH 169833) (bottom). All are from site 179. 


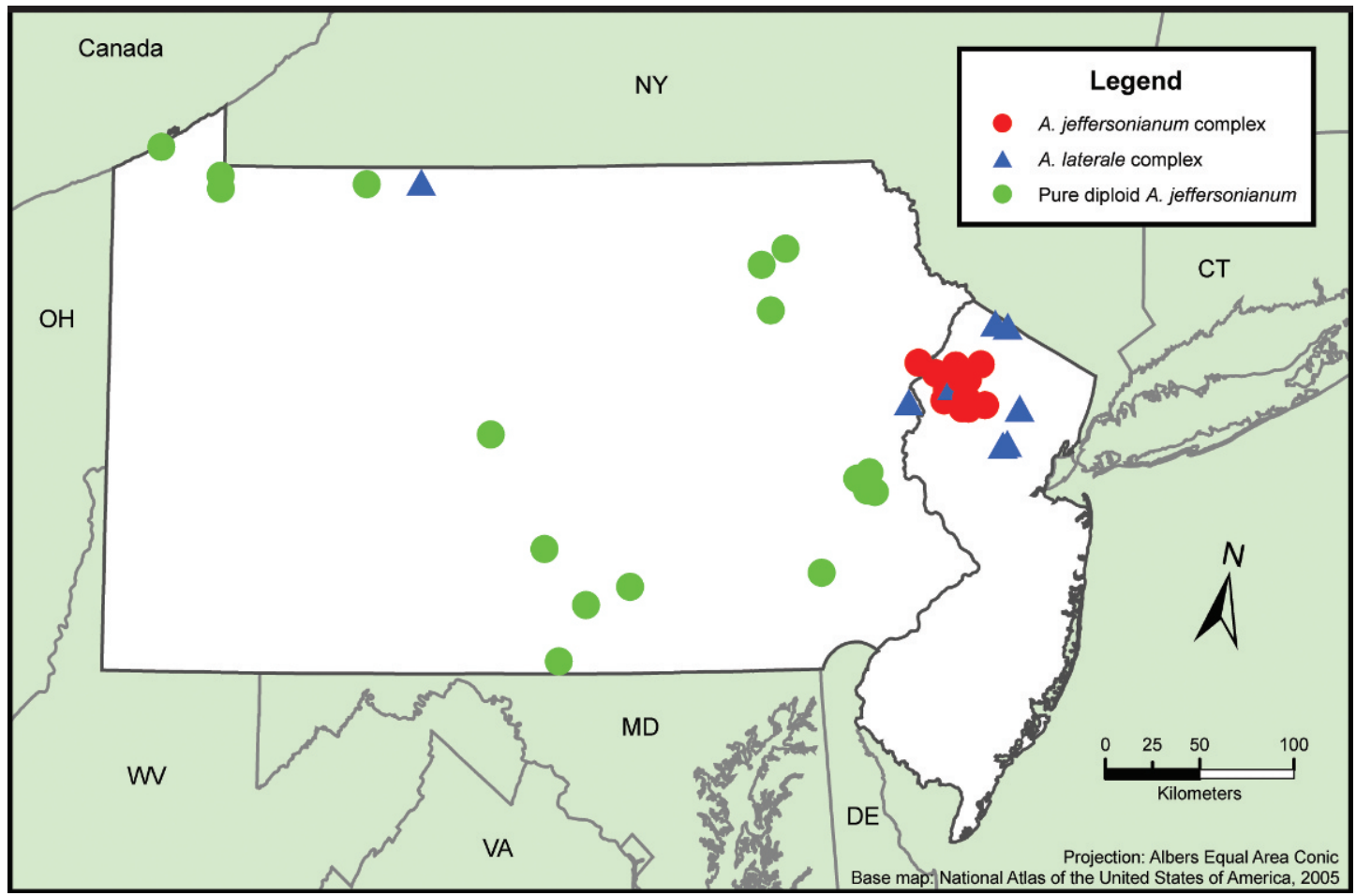

Fig. 4. Distribution of Ambystoma jeffersonianum, Ambystoma laterale, and unisexuals in New Jersey and Pennsylvania.

A. laterale, and only $A$. laterale $(\mathrm{n}=17)$ was found at site 150 . Unisexual LLJ were found with A. laterale in sites 151 (LL: $\mathrm{n}=7$; LLJ: $\mathrm{n}$ $=2$ ) and 152 (LL: $\mathrm{n}=8$; LLJ: $\mathrm{n}=2$ ). A single LLJ individual was collected at site 149. Other sites where we found only $A$. laterale were in western New York counties of Erie (site 146: n $=1$ ) and Niagara (site 147: $\mathrm{n}=7$ ); in the northeastern county of Essex (site 153: $\mathrm{n}=1$ ); and in the southeastern counties of Dutchess (site 206: $\mathrm{n}=2$ ) and Putnam (site 210: $\mathrm{n}=5$ ), but with such small sample sizes, these sites could also contain unisexual individuals. Ambystoma jeffersonianum were found in populations throughout New York (fig. 5). Populations containing only $A$. jeffersonianum were Tompkins County (site 2: $\mathrm{n}=38$; site 120: $\mathrm{n}=14)$, Sullivan County (site 121: $\mathrm{n}=$ 20), and Otsego County (site 118: $\mathrm{n}=16$ ) in east-central New York. The single A. jeffersonianum specimens from site 116 in Ulster County is probably not a pure $A$. jeffersonianum site because site 116 is in the same drainage basin and is close to site 117 where
LJJ and LJJJ individuals were found. Other New York sites where only $A$. jeffersonianum were found were represented by only one or two individuals: Cattaraugus County in western New York (site 135: $\mathrm{n}=1$ ) and Columbia County (site 212: $\mathrm{n}=2$ ), as well as Westchester County (site 205: $\mathrm{n}=1$ ) in southeastern New York.

\section{Pennsylvania and Virginia}

Ambystoma laterale and unisexuals have not been reported to occur in Pennsylvania. As expected, most of the Pennsylvania sites contained only $A$. jeffersonianum, but unisexual LJJ $(\mathrm{n}=7)$ and LJJJ $(\mathrm{n}=1)$ were found with $A$. jeffersonianum $(\mathrm{n}=9)$ at site 179 in Monroe County in eastern Pennsylvania. Ambystoma jeffersonianum, LJJ, and LJJJ from site 179 are shown in figure 3 . We also found one LJ and three LLJ unisexual individuals at site 174 in Northampton County, which is also in eastern Pennsylvania. Individual $A$. laterale were not found at site 


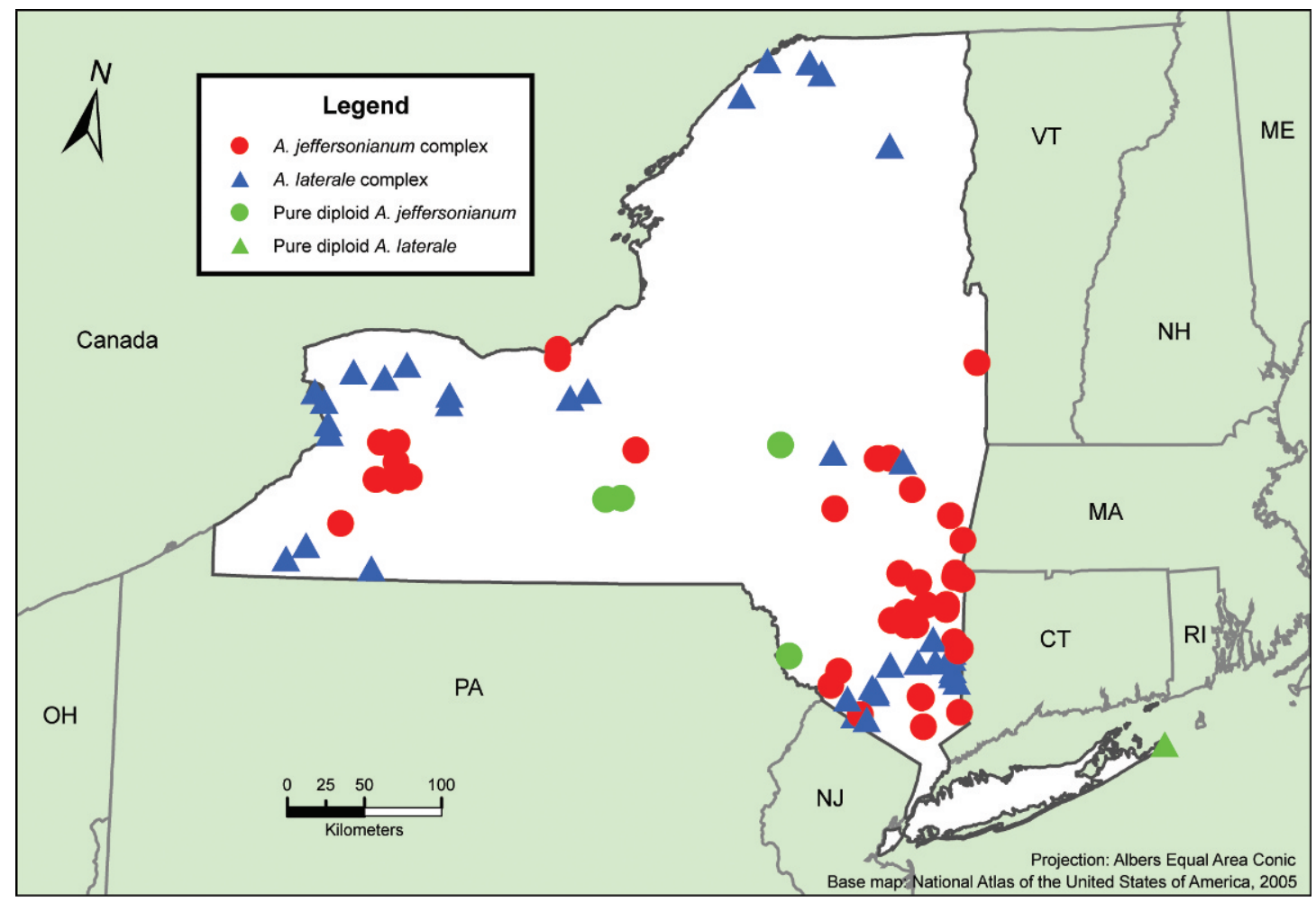

Fig. 5. Distribution of Ambystoma jeffersonianum, Ambystoma laterale, and unisexuals in New York.

174 but is assumed to be the sperm donor for these unisexuals $(62.5 \% \mathrm{~L}$, table 4$)$. Ambystoma laterale $(\mathrm{n}=45)$ was found in McKean County (site 189), in north-central Pennsylvania, with LLJ $(n=20)$ unisexuals. Ambystoma laterale, LJ, and LLJ individuals from these sites are provided in figure 2. The distribution of sexual and unisexual individuals that we found in Pennsylvania is plotted in fig. 4. Only four individuals were sampled from one site (site 190) in Virginia. They were all $A$. jeffersonianum.

\section{DISCUSSION}

We found a lower ratio of unisexual to sexual individuals than were found by Bogart and Klemens (1997) in populations from New England and mostly eastern New York. We can account for the discrepancy because, in the present study, we sampled large numbers of $A$. jeffersonianum from populations in Pennsylvania where unisexuals probably do not exist and we sampled some more northern populations in New York where unisexuals were found to be at a lower frequency than $A$. laterale. But, in keeping with our previous study, in most populations where unisexuals coexist with sexual individuals, the unisexuals are still more numerous. During the course of this study, we visited some of the same sites in different years to increase our sampling of populations to find rare genomotypes, which can confirm the absence or presence of sexual and unisexual individuals. However, this was not always possible, especially if the salamanders were rare or difficult to collect and many sites are represented by one or two individuals. Because the unisexuals require a sperm donor to successfully reproduce, if a sexual individual was not encountered, we predicted the presence of $A$. laterale or $A$. jeffersonianum based on the genomotypes of the unisexuals. It was not possible to predict the absence or the presence of unisexuals. The possibility exists that a population might change over time or 


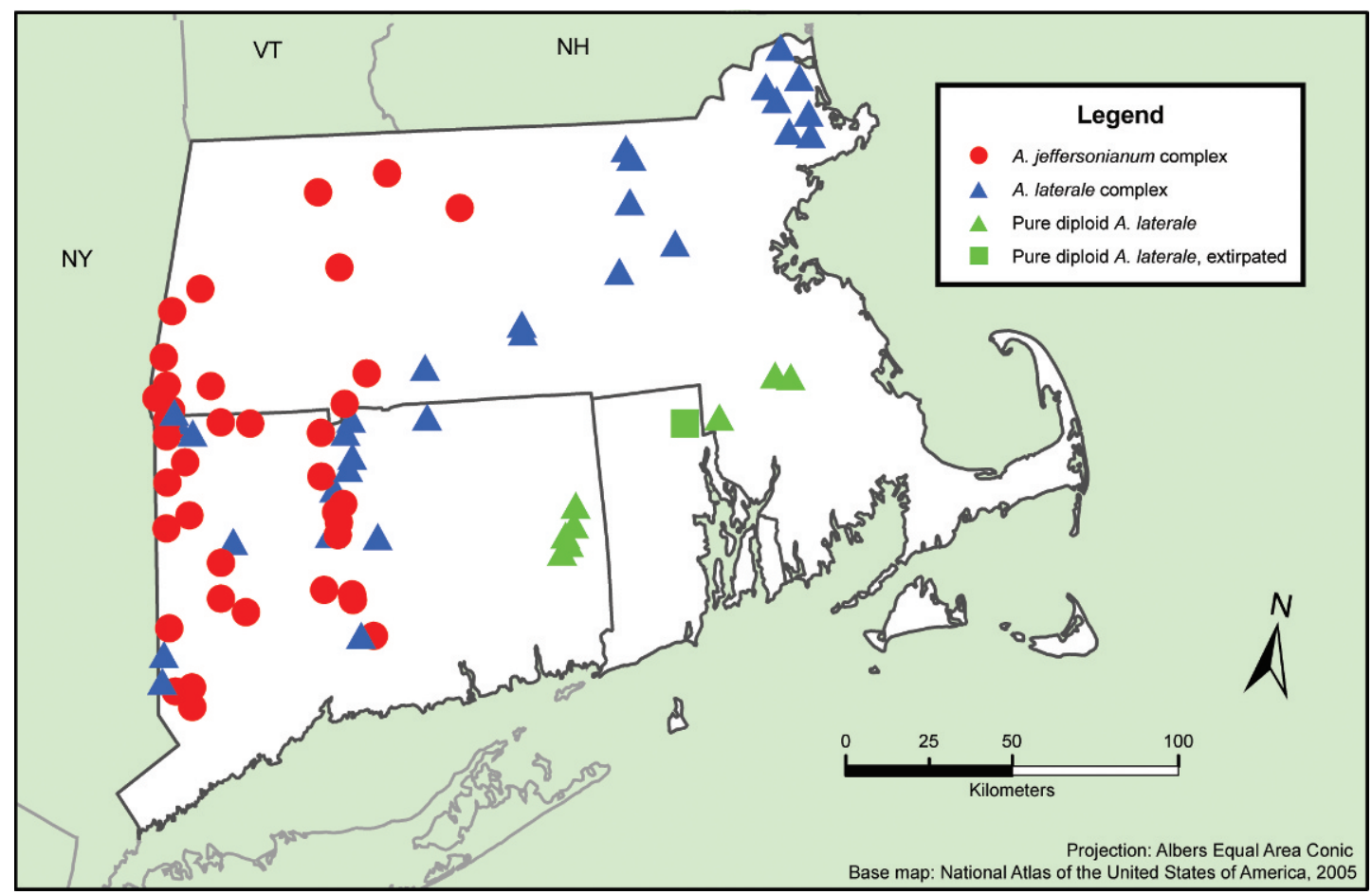

Fig. 6. Distribution of Ambystoma jeffersonianum, Ambystoma laterale, and unisexuals in Connecticut, Massachusetts, and Rhode Island.

that our sampling was biased. Eight of the 118 sites (marked $\dagger$ in table 4) sampled in the present study were also sampled for our earlier study (Bogart and Klemens, 1997). The species and genomotypes of the unisexuals were similar for most of the resampled populations. Increasing the sample size from three (Bogart and Klemens, 1997) to 38 individual $A$. jeffersonianum at site 2 in western New York supported our earlier contention that unisexuals are absent at that site. We did find $A$. jeffersonianum in Hartford County at Granby (site 195) and King Phillip Mountain near Simsbury (site 196), but we also found $A$. laterale at East Granby (site 197) and Farmington (site 198) near Rattlesnake Mountain. The Burnt Hill site (site 199), also near Farmington, yielded a single LJJ. A site near Wethersfield (site 201) was unusual as we found only seven diploid LJ unisexuals and, therefore, we can not predict a putative sperm donor for that site. Evidently, both sexual species are present and in close proximity in this region of Hartford County.

\section{No Apparent Syntopic Association of the Sexual Species}

Ambystoma laterale and A. jeffersonianum were not found together in any of the 118 sites and there were only four sites where both LLJ and LJJ were found (sites 108 and 113 in New York, site 161 in New Jersey, and site 197 in Connecticut) (appendix 1; table 4). Both New York sites were in Orange County. At site 108 we found LJJ $(n=6)$, LLJ $(n=17)$, LLLJ ( $=3$ ), and LJJJ $(\mathrm{n}=3)$ unisexual individuals. Ambystoma laterale $(\mathrm{n}=14)$ was the only sperm donor collected at that site, but the LJJ triploids and the LJJJ tetraploids indicate the sympatric presence of $A$. jeffersonianum. Based on egg mass data ( $\mathrm{Bi}$ and Bogart, 2006; Bi et al., 2007; Bogart et al., 2007) tetraploids are often found among the triploid offspring from individual triploid unisexuals through ploidy elevation that may be mediated by elevated temperature (Bogart et al., 1989). The additional J genome in the LJJJ tetraploids would be an indication that $A$. 


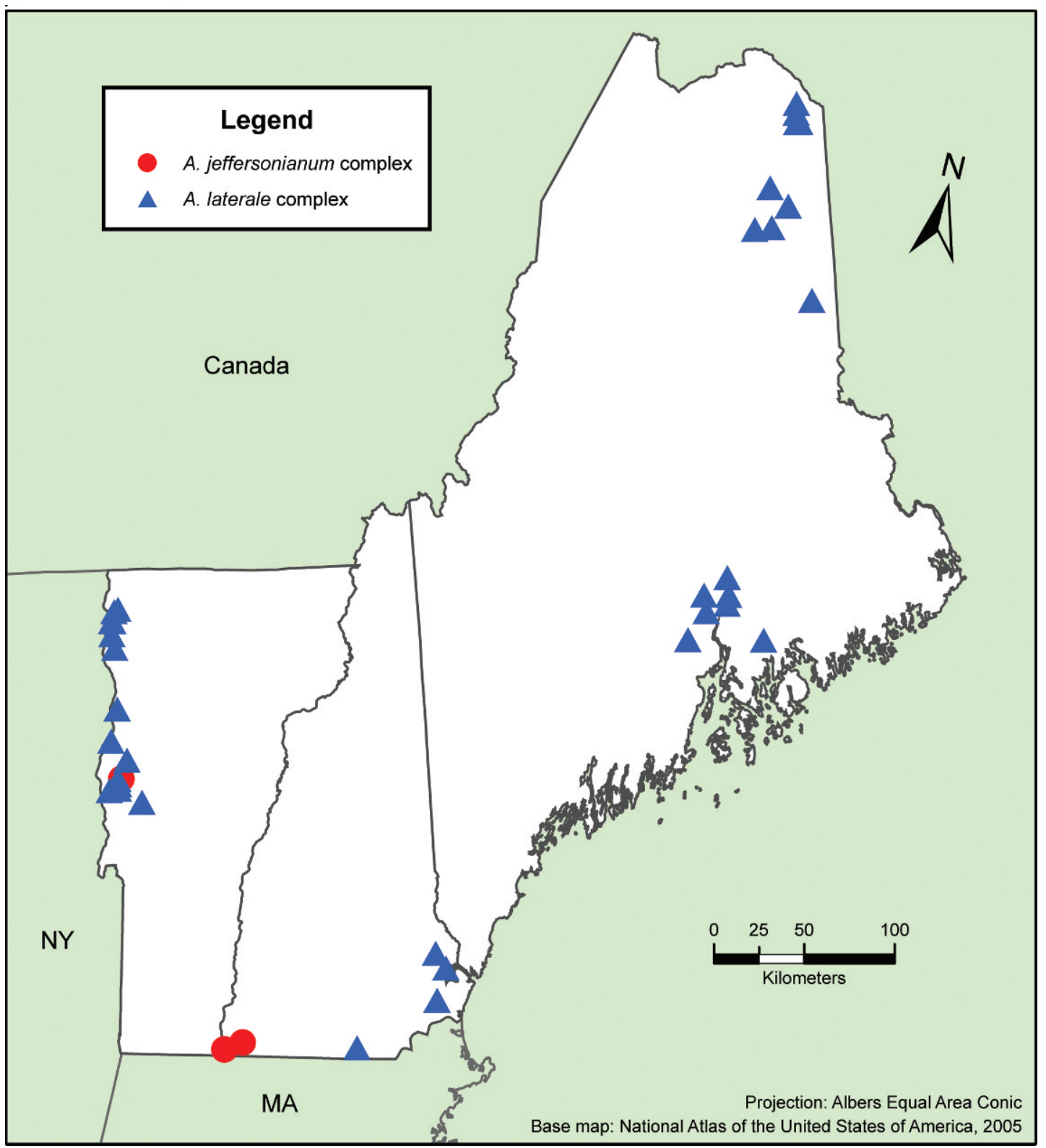

Fig. 7. Distribution of Ambystoma jeffersonianum, Ambystoma laterale, and unisexuals in Maine, New Hampshire, and Vermont.

jeffersonianum was the sperm donor if the LJJJ unisexual individuals were derived from a triploid LJJ female, but the tetraploids could have been gynogenetic offspring from a tetraploid LJJJ female. Ambystoma laterale and associated LLJ and LLLJ unisexuals were found in other Orange County sites 107, 110,
111, and 113. Ambystoma jeffersonianum, LJ, and LJJ were found in Orange County (site 114). If $A$. jeffersonianum does occur at site 108 , it must be less common than A. laterale and that population might demonstrate the phenomenon of one sexual species displacing the other over time. Making a case for the 


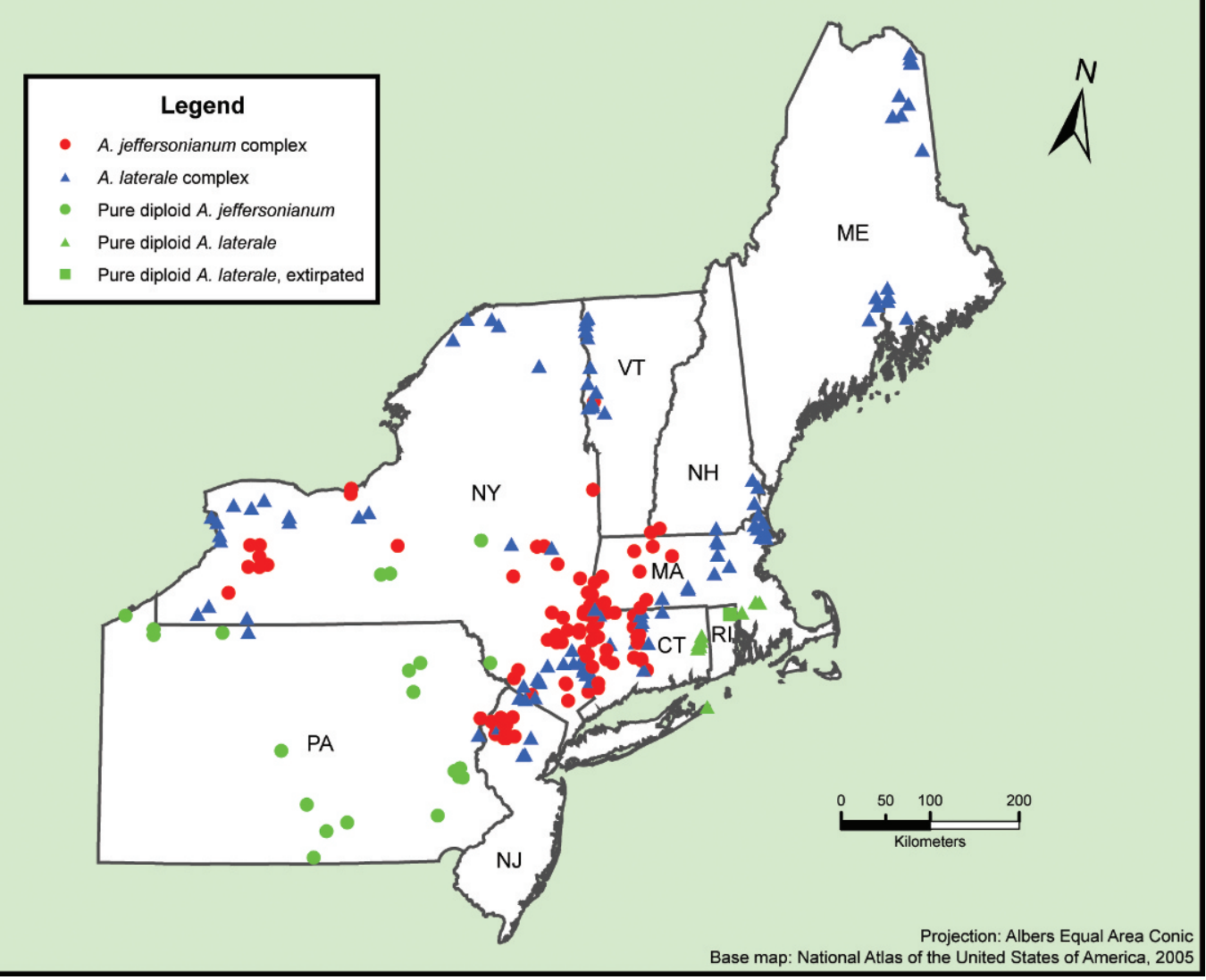
U.S.

Fig. 8. Distribution of Ambystoma jeffersonianum, Ambystoma laterale, and unisexuals in northeastern

presence of $A$. jeffersonianum in the other Orange County site that is dominated by $A$. laterale (site 113) is more tenuous because, of the 43 specimens collected at that site, there was only one LJJ individual and no LJJJ tetraploids. Dispersal of LJJ unisexuals into an A. laterale/unisexual site would be a possible alternate explanation.

Neither sexual species was collected at site 161 in Warren County, New Jersey, where LLJ $(n=3)$ and LJJ $(n=7)$ were found. The tetraploid LJJJ that was also collected at site 161 provides similar evidence that $A$. jeffersonianum is most likely the sperm donor. In that same county, both $A$. laterale $(\mathrm{n}=5)$ (site 162) and $A$. jeffersonianum (site 159: $\mathrm{n}=2$; site 163 : $\mathrm{n}=3$ ) were also collected. Based on our collection, the Hartford, Connecticut, site (198) is an $A$. laterale/unisexual site because $A$. laterale $(\mathrm{n}=4)$ and three LLLJ tetraploids were collected, but the three LJJ unisexuals that were also collected could indicate that this site also has $A$. jeffersonianum as have been found in other Hartford County sites (195 and 196).

From the relatively few examples of the sympatric occurrence of LLJ and LJJ, it is evident that all such instances exist only where $A$. laterale and $A$. jeffersonianum are parapatric and possibly sympatric over time and space. In a southern Ontario population where $A$. laterale and $A$. jeffersonianum were found to be breeding in the same pond, LJJ and LLJ unisexuals shared microsatellite alleles (Bogart et al., 2007). In that study, A. laterale was found to be an acceptable sperm donor for LJJ unisexual females because LLJJ larvae were encountered in the same egg mass with LJJ larvae. Through genome replacement, the 
switch from LJJ to LLJ or vice versa is probably a rapid and widespread phenomenon within the unisexual complex (Bogart, 2003) and would explain our observations in the few populations where this process is probably occurring.

\section{Diploid and Tetraploid Unisexuals}

Most unisexuals in this and previous studies (Uzzell, 1964; Bogart et al., 1987; Bogart and Klemens, 1997) are triploid. Tetraploids can be produced in the laboratory (Bogart et al., 1989) and are found to be derived in nature from triploid individuals (Bogart et al., 2007) through the process of ploidy elevation where a sperm nucleus is incorporated in an unreduced, triploid egg. We found tetraploids in scattered populations (table 4) and triploids were always found in the same populations. Diploid unisexuals are much more difficult to explain. They, too, are scattered in various populations (table 4) and, in a few populations, they were more numerous than the triploids (e.g., sites 123, 125, 137, 214) or were the only unisexual genomotype found (sites 134, 201). They occur in sites with either $A$. laterale or A. jeffersonianum. Diploid LJ and triploid LLJ or LJJ unisexuals share the same microsatellite alleles when they are sympatric and diploid LJ unisexuals can produce triploids through ploidy elevation events (Bogart et al., 2007). Ploidy reductional events probably occur from triploid to diploid unisexuals, but empirical evidence is lacking. Two very interesting sites (sites 123 and 125) in Schoharie County, New York, have LJ and LLJ unisexuals as well as Ambystoma jeffersonianum. The other Schoharie site (124), which also has $A$. jeffersonianum and LJ unisexuals, has the expected LJJ unisexual genomotype. We did not find any A. laterale in Schoharie County. Nor did we find A. laterale in Albany (site 126) or Otsego (site 118) counties that are, respectively, north and south of Schoharie County. However, in our 1997 paper we found $A$. laterale and its associated hybrids in Albany County (site 3 ). Only A. jeffersonia$\operatorname{num}(\mathrm{n}=16)$ was found at site 118 . We found A. jeffersonianum $(\mathrm{n}=4)$, as well as $\mathrm{LJ}(\mathrm{n}=$ $5)$, LJJ $(\mathrm{n}=18)$, and LJJJ $(\mathrm{n}=2)$ unisexuals at site 126 .

\section{"UniseXual” Males}

We found a very low frequency of "unisexual" males in this investigation and in our earlier study (Bogart and Klemens, 1997). They were diploid LJ (sites 114, 122), triploid LLJ (sites 108, 112, 152, 162), triploid LJJ (Site 165), and tetraploid LLLJ (site 148). Such males are probably sterile based on the limited cytological evidence of mieotic chromosomes (Bogart, 2003). It is possible that an answer to the rare finding of "unisexual" males may be found among the chromosome translocations and recombinations ( $\mathrm{Bi}$ and Bogart, 2006; Bi et al., 2007) that may involve sex-determining genes, but sex-determining genes have not been mapped in unisexuals to any chromosome region or, indeed, to any chromosome. The possibility exists that these rare males, even if they are sterile, could stimulate gynogenetic development of unisexual eggs. This might be important if other male sexual sperm donors are not available in a breeding pond. Obviously, natural selection should favor the production of female unisexuals.

Homozygosity and Reversals of Diagnostic Alleles in UniseXual Individuals

Identification of unisexual genomotypes is based on observations of allozymes that are diagnostic for $A$. laterale and $A$. jeffersonianum and demonstrate the appropriate dosage in diploids and polyploids. Homozygous allozyme patterns at these loci identify the sexual species and a reversed dosage pattern of allozymes at a locus would misidentify a LLJ as an LJJ or vice versa. Misidentification and ambiguity is alleviated by using several loci that possess diagnostic allozymes, so it is possible to recognize that reversals can and do occur, but if an individual had a reversed pattern for several loci it could be misidentified. If unisexuals were identical, genetic clones, as has been previously proposed (Uzzell, 1964; Macgregor and Uzzell, 1964; Uzzell and Goldblatt, 1967), a unisexual lineage could not revert from a homozygous to a heterozygous condition and reversed patterns, once attained, would be fixed (Asher and Nace, 1971). Fluorescent genomic in situ hybridization (GISH) of unisexual 
chromosomes (Bi and Bogart, 2006; Bi et al., 2007) and microsatellite analyses (Bogart et al., 2007) clearly show that unisexuals are not clones and, based on chromosomal translocations and recombinations, may be expected to demonstrate gene restructuring. Thus, we may be surprised that observed homozygous and reversed-allozyme alleles are not more common and widespread than the few instances that we found in the present study (tables 5 and 6) and in our earlier study (Bogart and Klemens, 1997). We suspect that natural selection eliminates many such mutational events and helps to explain the embryonic mortality that is ubiquitous in unisexual populations. Unisexual kleptogens swap genomes with sexual sperm donors (Bi et al., 2008). This process may be essential for unisexuals to recover from detrimental genetic restructuring and consequentially, maintain a fixed heterozygous genotype that is most commonly found in unisexual individuals and likely has a selective advantage.

\section{Rare Alleles}

Loci chosen to identify the sexual and unisexual individuals were loci that were mostly monomorphic for alternate alleles in $A$. jeffersonianum and $A$. laterale, but these same loci are not considered to be very conservative in other vertebrates. When sampling individuals from many populations over a large range we should expect to find polymorphism and population specific "private" alleles. The very low frequency of some rare alleles may be attributed to mutational events and especially if a single rare allele is found in only one individual. Finding rare alleles among sexual and unisexual individuals in the same populations adds credence to kleptogenesis.

Some rare alleles that were found in both $A$. jeffersonianum and $A$. laterale such as Aat- ${ }^{-50}, \mathrm{Mdh}^{-1}{ }^{200}$ or $\mathrm{Mpi}^{80}$ (table 3) may be alleles that have been passed on from a common ancestor and are maintained at a low frequency in both species. A rare allele demonstrates that a diploid-triploid unisexual relationship exists in site 130 . The only unisexuals that were found to have Idh- $1^{160}$ were LJ at site 130 and LJJ from sites 130 and
131. That allele was not found in A. jeffersonianum from those sites but was found in individual $A$. jeffersonianum in sites 118 and 204 and in A. laterale from sites 113 and 189 . Ldh-1 ${ }^{78}$ was not found by Bogart and Klemens (1997). We found this allele in $A$. laterale populations (sites 127, 134, 136, 143, 145) in western New York and in site 189 in north-central Pennsylvania. The unisexual LLJ individuals from the Pennsylvania site that also had this allele were homozygous for Ldh $-1{ }^{78}$. LLJ homozygotes for this allele were also found in site 148 close to Lake Erie in New York. The relatively high frequency of Ldh- $1^{78}$ in western New York $A$. laterale individuals is a clear indication that $A$. laterale genomes that contain this allele have been used to replace $\mathrm{L}$ genomes in sympatric unisexuals. The homozygous condition of Ldh $-1^{78}$ in the unisexuals demonstrates that the diagnostic $A$. jeffersonianum Ldh- ${ }^{88}$ allele was probably lost through genome translocations or recombination or is no longer functional in the homozygous unisexuals.

\section{SUMMARY AND CONCLUSIONS}

Identification of Ambystoma jeffersonianum and $A$. laterale and their breeding ponds in the northeastern United States in this, and in our earlier, study should benefit conservation efforts to preserve and maintain these species. Populations that we examined as many as 20 years ago may no longer exist. The interaction of unisexuals with the sexual species is a fascinating evolutionary phenomenon that is probably unique. Unisexual kleptogens take genomes from either sexual species and are usually more numerous in sympatric association with a sexual species. Unisexuals were not found in most of the $A$. jeffersonianum populations in Pennsylvania and are less numerous than A. laterale in northern New York populations. Perhaps the unisexuals are recent immigrants in those New York populations and have not yet dispersed throughout Pennsylvania. The fact that no unisexuals were found with $A$. laterale in Long Island (New York), eastern Connecticut, and southeastern Massachusetts (Bogart and Klemens, 1997) could also be the result of isolation of those populations prior to unisexual dispersion. 
Although no new samples were obtained from Vermont, New Hampshire, and Maine, we have included the ranges of known sexual and unisexual individuals from those states in figure 7 from our earlier investigation (Bogart and Klemens, 1997). Figure 8 includes the known ranges for these salamanders in the northeastern United States from the present and previous study.

In addition to $A$. laterale and $A$. jeffersonianum, unisexuals use other species ( $A$. texanum, A. tigrinum) as sperm donors in Ohio, Illinois, Indiana, and Michigan (Kraus, 1995; Morris, 1985; Kraus and Petranka, 1989; Selander, 1994; Bogart, 2003). Distinguishing sexual and unisexual individuals in those states is complicated because $A$. texanum and $A$. laterale have the same allozymes for some of the diagnostic loci (Bogart et al., 1987). It is likely that isozyme electrophoresis will be replaced as a technique to identify these salamander species and their associated unisexuals. Sequences of mitochondrial DNA show all of the sexual species to be distinctive and distinctly different from the unisexuals (Hedges et al., 1992; Bogart, 2003; Bogart et al., 2007). Microsatellite DNA alleles can identify $A$. jeffersonianum, A. laterale and the unisexuals that use those species as sperm donors (Julian et al., 2003; Ramsden et al., 2006; Bogart et al., 2007). Because these DNA techniques do not require sacrificing individuals, there are obvious advantages when attempting to identify rare, threatened, or endangered salamanders. Identifying the species and unisexuals is a prerequisite to protecting the species and their habitat. Subsequent temporal monitoring will be necessary to understand the interactions of the species and the unisexual associates.

\section{ACKNOWLEDGMENTS}

We wish to thank the many friends, colleagues, and students who helped us with the collecting of samples, for logistical support in the field, and assisting in the laboratory. Julian D. Avery, Michael Bernard, David H. Black, Randy Cassell, Amy R. Croushore, Peter Ducey, the late Leigh Gillett, Brian Gray, Hank J. Gruner, Christy Hicks, Art Hulse, Steve Kahl, Ed Kanze, Andy Keal, Daniel S. Klemens, Robert M. Klemens, Rick Koval,
Mark Lethaby, George Logan,, Michael Losito, Tim Maret, Diane Murphy, Linda Ordiway, Damon Oscarson, Ed Pawlak, Dennis Quinn, Chris J. Raithel, Kenneth Roblee, Brandon M. Ruhe, Kevin J. Ryan, Jason Tesauro, Peter R. Warny, and Kristian Whiteleather assisted with the collection of salamanders. Dave Britton, Heather Lynn, Lesley Rye, Sara Wick, and Karlee Thomas all helped to process salamanders and assisted with the electrophoretic analyses. Karine Bériault measured blood cells and performed much of the flow cytometry. Dan Noble and Ke Bi made helpful comments on an earlier manuscript. We wish to especially thank Danielle T. LaBruna for creating the distribution maps and Kevin J. Ryan for assistance in rearing larval salamanders. We acknowledge use of the collections of the American Museum of Natural History (AMNH). All samples were collected under provisions of various state collecting permits and licenses and are on file at the AMNH. All laboratory procedures followed approved Animal Care Protocols from the University of Guelph Animal Care Committee. Financial support for portions of this study was provided by the Wildlife Conservation Society and the National Science and Engineering Research Council of Canada.

\section{REFERENCES}

Anderson, J.D., and R.V. Giacosie. 1967. Ambystoma laterale in New Jersey. Herpetologica 23: 109-111.

Asher, J.H., and G.W. Nace. 1971. The genetic structure and evolutionary fate of parthenogenetic amphibian populations as determined by Markovian analysis. American Zoologist 11: 381-398.

Austin, N.E., and J.P. Bogart. 1982. Erythrocyte area and ploidy determination in the salamanders of the Ambystoma jeffersonianum complex. Copeia 1982: 485-488.

Bi, K., and J.P. Bogart. 2006. Identification of intergenomic recombinations in unisexual salamanders of the genus Ambystoma by genomic in situ hybridization. Cytogenetic and Genome Research 112: 307-312.

Bi, K., J.P. Bogart, and J. Fu. 2007. Intergenomic translocations in unisexual salamanders of the genus Ambystoma (Amphibia, Caudata). Cytogenetic and Genome Research 116: 289-297.

Bi, K., J.P. Bogart, and J. Fu. 2008. The prevalence of genome replacement in unisexual salamanders of the genus Ambystoma (Amphibia, Caudata) revealed by nuclear gene genealogy. 
BMC Evolutionary Biology 8: 158. (doi:10.1186/ 1471-2148-8-158).

Bogart, J.P. 1982. Ploidy and genetic diversity in Ontario salamanders of the Ambystoma jeffersonianum complex revealed through and electrophoretic examination of larvae. Canadian Journal of Zoology 60: 848-855.

Bogart, J.P. 1989. A mechanism for interspecific gene exchange via all-female salamander hybrids. In R.M. Dawley and J.P. Bogart (editors), Evolution and ecology of unisexual vertebrates. New York State Museum Bulletin 466: $170-179$.

Bogart, J.P. 2003. Genetics and systematics of hybrid species. In D.M. Sever (editor), Reproductive biology and phylogeny of Urodela, vol. 1, 109-134. Enfield, NH: M/s Science.

Bogart, J.P., K. Bi, J. Fu, D. Noble, and J. Niedzwiecki. 2007. Unisexual salamanders (genus Ambystoma) present a new reproductive mode for eukaryotes. Genome 50: 119-136.

Bogart, J.P., R.P. Elinson, and L.E. Licht. 1989. Temperature and sperm incorporation in polyploid salamanders. Science 246: 1032-1034.

Bogart, J.P., and M.W. Klemens. 1997. Hybrids and genetic interactions of mole salamanders (Ambystoma jeffersonianum and A. laterale) (Amphibia: Caudata) in New York and New England. American Museum Novitates 3218: $1-78$.

Bogart, J.P., and L.E. Licht. 1986. Reproduction and the origin of polyploids in hybrid salamanders of the genus Ambystoma. Canadian Journal of Genetics and Cytology 28: 605-617.

Bogart, J.P., L.E. Licht, M.J. Oldham, and S.J. Darbyshire. 1985. Electrophoretic identification of Ambystoma laterale and Ambystoma texanum as well as their diploid and triploid interspecific hybrids (Amphibia: Caudata) on Pelee Island, Ontario. Canadian Journal of Zoology 63: 340-347.

Bogart, J.P., L.A. Lowcock, C.W. Zeyl, and B.K. Mable. 1987. Genome constitution and reproductive biology of the Ambystoma hybrid salamanders on Kelleys Island in Lake Erie. Canadian Journal of Zoology 65: 2188-2201.

Clayton, J.W., and D.N. Tretiak. 1972. Aminecitrate buffers for $\mathrm{pH}$ control in starch gel electrophoresis. Journal of the Fisheries Research Board of Canada 29: 1169-1172.

Danzmann, R.G., and J.P. Bogart. 1982. Gene dosage effects on MDH isozyme expression in diploid, triploid, and tetraploid treefrogs of the genus Hyla. Journal of Heredity 73: 277-280.

Downs, F.L. 1978. Unisexual Ambystoma from the Bass Islands of Lake Erie. Occasional Papers of the Museum of Zoology University of Michigan 685: 1-36.
Drowne, F.P. 1905. The reptiles and batrachians of Rhode Island. Roger Williams Park Museum Monograph 15: 1-24.

Hedges, S.B., J.P. Bogart, and L.R. Maxson. 1992. Ancestry of unisexual salamanders. Nature 356: 708-710.

International Union of Biochemistry Nomenclature Committee (IUBC). 1984, Enzyme nomenclature. New York: Academic Press.

Julian, S.E., T.L. King, and W.K. Savage. 2003. Novel Jefferson salamander, Ambystoma jeffersonianum, microsatellite DNA markers detect population structure and hybrid complexes. Molecular Ecology Notes 3: 95-97.

Kezer, J., and S.K. Sessions. 1979. Chromosome variation in the plethodontid salamander Aneides ferreus. Chromosoma 71: 65-80.

Klemens, M.W. 1978. Variation and distribution of the turtle, Chrysemys picta, (Schneider), in Connecticut. MS thesis, University of Connecticut, Storrs, $60 \mathrm{pp}$.

Klemens, M.W. 1993. The amphibians and reptiles of Connecticut and adjacent regions. State Geological and Natural History Survey of Connecticut Bulletin 112: 1-318 + 32 plates.

Klemens, M.W. 2000. Amphibians and reptiles in Connecticut: a checklist with notes on conservation status, identification, and distribution. DEP Bulletin 32: 1-96, Hartford, CT: Connecticut Department of Environmental Protection, Environmental and Geographic Information Center.

Kraus, F. 1995. The conservation of unisexual vertebrate populations. Conservation Biology 9: 956-959.

Kraus, F., and J.W. Petranka. 1989. A new sibling species of Ambystoma from the Ohio River drainage. Copeia 1989: 94-110.

Lowcock, L.A. 1994. Biotype, genomotype, and genotype: variable effects of polyploidy and hybridity on ecological partitioning in a bisexual-unisexual community of salamanders. Canadian Journal of Zoology 72: 104-117.

Lowcock, L.A., and J.P. Bogart. 1989. Electrophoretic evidence for the multiple origins of triploidy in the Ambystoma lateralejeffersonianum complex. Canadian Journal of Zoology 67: 350-356.

Lowcock, L.A., L.E. Licht, and J.P. Bogart. 1987. Nomenclature in hybrid complexes of Ambystoma: no case for the erection of hybrid "species". Systematic Zoology 36: 328-336.

Macgregor, H.C., and T.M. Uzzell, Jr. 1964. Gynogenesis in salamanders related to Ambystoma jeffersonianum. Science 143: 1043-1045.

Morris, M.A. 1985. A hybrid Ambystoma platineum $\times$ A. tigrinum from Indiana. Herpetologica 41: 267-271. 
Niedzwiecki, J. 2005. Evolutionary history and hybridization of two mole salamander sister species from different habitats. Ph.D. thesis, University of Kentucky, Lexington.

Petranka, J.W. 1998. Salamanders of the United States and Canada. Washington, DC: Smithsonian Institution Press.

Ramsden, C., K. Bériault, and J.P. Bogart. 2006. A nonlethal method of identification of Ambystoma laterale, A. jeffersonianum, and sympatric unisexuals. Molecular Ecology Notes 6: 261-264.

Schultz, R.J. 1969. Hybridization, unisexuality, and polyploidy in the teleost Poeciliopsis (Poeciliidae) and other vertebrates. American Naturalist 103: 605-619.

Selander, R.K., M.H. Smith, S.Y. Yang, W.E. Johnson, and J.B. Gentry. 1971. Biochemical polymorphism and systematics in the genus Peromyscus. I. Variation in the Oldfield mouse (Peromyscus polionotus): studies in genetics IV. University of Texas Publication 7103: 49-90.

Selander, T.C. 1994. Reproduction of unisexual hybrid Ambystoma (Urodela: Ambystomatidae) in the absence of parental species. M.Sc. thesis, University of Dayton, Ohio.

Sessions, S.K. 1982. Cytogenetics of diploid and triploid salamanders of the Ambystoma jeffersonianum complex. Chromosoma 84: 599-621.

Shaffer, H.B., and M.L. McKnight. 1996. The polytypic species revisited: genetic differentiation and molecular phylogenetics of the tiger salamander Ambystoma tigrinum (Amphibia: Caudata) complex. Evolution 50: 417-433.

Taylor, A.S., and J.P. Bogart. 1990. Karyotypic analyses of four species of Ambystoma (Amphibia, Caudata) which have been implicated in the production of all-female hybrids. Genome 33: 837-844.

Uzzell, T.M. 1964. Relations of the diploid and triploid species of the diploid and triploid species of the Ambystoma jeffersonianum complex (Amphibia, Caudata). Copeia 1964: 257-300

Uzzell, T.M., and S.M. Goldblatt. 1967. Serum proteins of salamanders of the Ambystoma jeffersonianum complex. Copeia 1967: 602612 .

\section{APPENDIX 1}

Additional Individuals Collected from Previous Collection Sites Examined by Bogart and Klemens (1997)

Localities listed by site numbers where Ambystoma laterale (LL), A. jeffersonianum (JJ), and their diploid (LJ), triploid (LLJ or LJJ), and tetraploid (LLLJ, LLJJ, LJJJ) unisexual kleptogens were identified. New sites examined (sites 107-216) continue from the 106 sites examined by Bogart and Klemens (1997). Catalogue numbers refer to specimens that are deposited in the American Museum of Natural History (AMNH) and catalogue numbers of J. P. Bogart (JPB).

2) New York: Tompkins County: Dryden. $(\mathrm{n}=35)$. JJ (AMNH 160166-200).

7) New York: Orange County: Chester, Goose Pond Mountain, Seeley Brook. (n = 4). LLJ (AMNH 164593). LLLJ (AMNH 158825, AMNH 160339-40).

18) New York: Dutchess County: Pawling, Great Swamp, South of Rte. 55 overpass. (n = 5). LL (AMNH 159999160000). LJ (AMNH 165937). LLJ (AMNH 165994). LLJJ (AMNH 153121).

20) New York: Putnam County: Putnam Valley $(\mathrm{n}=2)$. LJJ (AMNH 169808-09).

28) Connecticut: Fairfield County: Redding. ( $n=1)$. LJJ (AMNH 158787).

42) Connecticut: Litchfield County: Bantam Lake. $(n=6)$. LL (AMNH 160056). LLJ (AMNH 166983-87).

60) Connecticut: Windham County: Plainfield. $(\mathrm{n}=2)$. LL (AMNH 169855-56).

66) Massachusetts: Bristol and Plymouth counties: Hockomock Swamp. $(\mathrm{n}=2)$. LL (AMNH 169355-56).

\section{NEW COLLECTION SITES WEST OF THE HUDSON RIVER New York}

107) Orange County: Blooming Grove, Youngs Brook. ( $\mathrm{n}=25)$. LL (AMNH 169885-93). LJ (AMNH 169913-16). LLJ (AMNH 169900-06). LLLJ (AMNH 169899, AMNH 169909-12).

108) Orange County: Amity. (n = 43). LL (AMNH 160041-54). LJ (AMNH 160248-54), LLJ (AMNH 160055, AMNH 160269-84). LJJ (AMNH 153118, AMNH 160330). LLLJ (AMNH 160341-43).

109) Orange County: Amity Uplands. ( $=9$ ). LJ (AMNH 169917-18). LJJ (AMNH 169849, AMNH 169918-21). LJJJ (AMNH 169922-24).

110) Orange County: Warwick, Wawayanda Creek - Bellvale Valley (n = 31). LL (AMNH 169369-84, AMNH 169882-84). LJ (AMNH 169395). LLJ (AMNH 165995, AMNH 169396-98, AMNH 169894-98). LLLJ (AMNH 169907-08). 


\section{APPENDIX 1}

(Continued)

111) Orange County: Goshen, Otter Kill floodplain swamp. $(\mathrm{n}=26)$. LL (AMNH 169386-94). LLJ (AMNH 169385, AMNH 169399-410, AMNH 169806). LLLJ (AMNH 169411-13).

112) Orange County: Wawayanda, vicinity of Echo Lake Rd. (n = 5). LJ (AMNH 169802). LLJ (AMNH 169803-05, AMNH 169807).

113) Orange County: Stewart State Forest $(n=43)$. LL (AMNH 162962, AMNH 164587-88, AMNH 162963-65). LJ (AMNH 162819-23, AMNH 162825-30, AMNH 162946, AMNH 164590-92, AMNH 164636-38). LLJ (AMNH 162942-45, AMNH 162947-49, AMNH 162951-59). LJJ (AMNH 162939). LLLJ (AMNH 162950).

Orange County 2 mi. ESE Mount Hope. $(n=46)$. JJ (AMNH 153123-59, AMNH 160239-45). LJ (AMNH153110). LJJ (AMNH 153112).

115) Ulster County: between Butterville and Bonticou Crag. (n = 3). JJ (AMNH 153122). LJJ (AMNH 153116, AMNH 160337).

116) Ulster County: Lloyd, Black Creek floodplain. $(\mathrm{n}=1)$. JJ (AMNH 169460).

117) Ulster County: Esopus, Black Creek Preserve. $(n=9)$. LJJ (AMNH 169451-55). LJJJ (AMNH 169456-59).

118) Otsego County: Oaks Creek drainage. $(\mathrm{n}=16)$. JJ (AMNH 160223-38).

119) Cortland County: Scott Township. $(n=7)$. JJ (AMNH 160222). LJJ (AMNH 160331-36).

120) Tompkins County: Ithaca, Bull Pasture Pond. $(n=14)$. JJ (AMNH 163376-89).

121) Sullivan County: Tusten. $(\mathrm{n}=20)$. JJ (AMNH 160202-21).

122) Sullivan County: Bashakill State Wildlife Management Area. (n = 7). LJ (AMNH 153107-09, AMNH 153119). LJJ (AMNH 153113-15).

123) Schoharie County: near South Gilboa Station along Rte. 23. (n = 14) JJ (AMNH 160201). LJ (AMNH 153111, AMNH 162832-34, AMNH 165938-44). LLJ (AMNH 165996). LJJ (AMNH 162835).

124) Schoharie County: Wright site. $(\mathrm{n}=11)$. JJ (AMNH 169823). LJ (AMNH 169810-12). LJJ (AMNH 169816-22).

125) Schoharie County: Seward. $(n=5)$. JJ (AMNH 169824-25). LJ (AMNH 169813-14). LLJ (AMNH 169815).

126) Albany County: Knox. ( $=30)$. JJ (AMNH 169797-801). LJ (AMNH 169772-76). LJJ (AMNH 169777-94). LJJJ (AMNH 169795-96).

127) Seneca/Wayne Counties: Montezuma Marsh. $(n=51)$. LL (AMNH 160001-40, AMNH 162968). LLJ (AMNH 160257-66).

128) Cayuga County Mentz. (n=1). LLJ (AMNH 160268).

129) Genesee County: Alabama, Orchard Swamp. (n = 12). LL (AMNH 162969-70, AMNH 165869-74). LLJ (AMNH 162961, AMNH 165997-99).

130) Wayne County Huron, Lummisville Road. (n = 13). JJ (AMNH 163372, AMNH 165826-30). LJ (AMNH 165945). LJJ (AMNH 162938, AMNH 165953-57).

131) Wayne County: Huron, Lake Ontario wetland. $(n=6)$. JJ (AMNH 165831). LJJ (AMNH 165958-62).

132) Monroe County: Wheatland, Blue Pond. ( $\mathrm{n}=2$ ). LL (AMNH 162966). LLJ (AMNH 162960).

133) Orleans County: Barre, Culver Road. ( $=7$ ). LL (AMNH 165875-77). LLJ (AMNH 166000-03).

134) Livingston County: Caledonia, Cement Plant Pond. $(\mathrm{n}=2)$. LL (AMNH 162967). LJ (AMNH 162831).

135) Cattaraugus County: Mansfield. $(\mathrm{n}=1)$. JJ (AMNH 163467).

136) Cattaraugus County: Conewango Creek. ( $\mathrm{n}=18)$. LL (AMNH 165878-84). LLJ (AMNH 166004-14).

137) Wyoming County: Arcade. $(n=12)$. JJ (JPB 31109, AMNH 163462-66, AMNH 165832-33, AMNH 167064). LJ (AMNH 167065-66). LJJ (AMNH 167067).

138) Wyoming County: Eagle, West Hill Road. (n = 4). JJ (AMNH 167068-69). LJ (AMNH 167070). LJJ (AMNH 167071).

139) Wyoming County: Pike, Safford Road. (n = 1). LJJ (AMNH 167072).

140) Wyoming County: Wethersfield. $(n=4)$. JJ (AMNH 169999). LJJ (AMNH 169998, AMNH 170000-01).

141) Wyoming County: Orangeville. $(\mathrm{n}=3)$. LJJ (AMNH 170002-04).

142) Wyoming County: Sheldon. $(\mathrm{n}=1)$. LJJ (AMNH 170005).

143) Erie County: Buffalo, RR yards between Tifft Street and Buffalo River. (n=17). LL (AMNH 162971, AMNH 167021-32). LLJ (AMNH 167033-36).

144) Erie County: Grand Island. $(\mathrm{n}=10)$. LL (AMNH 165885-91). LLJ (AMNH 166015-17).

145) Erie County: Lackawanna, South branch of Smoke Creek. (n = 19). LL (AMNH 167037-40). LLJ (AMNH 167041-55).

146) Erie County: Tonawanda, Kenmore. $(n=1)$. LL (AMNH 167056).

147) Niagara County: Royalton. $(\mathrm{n}=7)$. LL (AMNH 167057-63).

148) Chautauqua County: Poland, Conewango Creek. $(\mathrm{n}=9)$. LLJ (AMNH 167012-19). LLLJ (AMNH 167020).

149) St. Lawrence County: Lawrence. $(\mathrm{n}=1)$. LLJ (AMNH 169446). 


\section{APPENDIX 1}

(Continued)

150) St. Lawrence County: Brasher, Bush Rd. (n = 17). LL (AMNH 169414-30).

151) St. Lawrence County: Waddington-Louisville town line, Town Line Rd. (n = 9). LL (AMNH 169431-37). LLJ (AMNH 169447-48).

152) St. Lawrence County: Lisbon, Swamp Rd. $(\mathrm{n}=10)$. LL (AMNH 169438-45). LLJ (AMNH 169449-50).

153) Essex County: St. Armand, Adirondack Park. $(\mathrm{n}=1)$. LL (AMNH 169368).

New Jersey

154) Morris and Somerset Counties: Great Swamp. $(\mathrm{n}=13)$. LL (AMNH 160057-68, AMNH 160071).

155) Morris County: Troy Meadows. $(\mathrm{n}=10)$. LL (AMNH 160069-70). LLJ (AMNH 160285-91). LLLJ (AMNH 160338).

156) Morris County: Budd Lake. (n = 13). JJ (AMNH 160162-64, AMNH 163366-67, AMNH 163369). LJ (AMNH 162816-18). LJJ (AMNH 162925, AMNH 162933-35).

157) Morris County: Stephens State Park. $(\mathrm{n}=8$ ). JJ (AMNH 163368). LJJ (AMNH 162926-32).

158) Morris County: Berkshire Valley Wildlife Management Area. $(n=13)$. JJ (AMNH 165834-40). LJ (AMNH 165946). LJJ (AMNH 165963-67).

159) Warren County: Hardwick Twp. (n = 9). JJ (AMNH 163370-71). LJ (AMNH 160225). LJJ (AMNH 160322-24, AMNH 162922-24).

160) Warren County: Jenny Jump Sink. $(\mathrm{n}=4)$. LJJ (AMNH 165968-71).

161) Warren County: Frelinghuysen Twp., Glovers Pond. (n=11). LLJ (AMNH 166999-01). LJJ (AMNH 16699197). LJJJ (AMNH 166998).

162) Warren County: Frelinghuysen Twp., Bear Creek. ( $\mathrm{n}=16$ ). LL (AMNH 166988-90, AMNH 169357-58). LLJ (AMNH 167002-03, AMNH 169359-66). LLLJ (AMNH 167004).

163) Sussex County: Swartswood. (n = 12). JJ (AMNH 163361-63). LJJ (AMNH 160329, AMNH 162890-96, AMNH 162902).

164) Sussex County: Kittatinny Valley State Park. $(\mathrm{n}=12)$. JJ (AMNH 160165, AMNH 163354-57, AMNH 162900). LJJ (AMNH 162886, AMNH 162897-99, AMNH 162901, AMNH 163554).

165) Sussex County: Fredon Twp., Whittingham Wildlife Management Area. (n = 17). LJJ (AMNH 160321, AMNH 160325-28, AMNH 162910-21).

166) Sussex County: Andover, north end of Stickle Pond. ( $\mathrm{n}=15)$. JJ (AMNH 163352-53, AMNH 163365). LJJ (AMNH 162881-85, AMNH 162903-09).

167) Sussex County: Byram Twp., Allamuchy State Park. (n = 6). JJ (AMNH 163358-60). LJJ (AMNH 162887-89).

168) Sussex County: Wallkill River Drainage $(\mathrm{n}=38$ ). LL (AMNH 160072-79, AMNH 169925). LLJ (AMNH 160292-309, AMNH 160311-17, AMNH 160320). LLLJ (AMNH 160310, AMNH 160318-19)

169) Sussex County: Sparta Twp., Sussex County Votech Pond. (n = 7). JJ (AMNH 167005). LJ (AMNH 167006). LJJ (AMNH 167007-11).

\section{Pennsylvania}

170) Lehigh County: Emmaus. $(\mathrm{n}=33)$. JJ (AMNH 160080-112).

171) Lehigh County: Center Valley. $(\mathrm{n}=10)$. JJ (AMNH 165841-50).

172) Lehigh County: Salisbury Twp. $(\mathrm{n}=9)$. JJ (AMNH 169483-91).

173) Lehigh County: Upper Saucon Twp. ( $\mathrm{n}=17)$. JJ (AMNH 169492-506, AMNH 169929-30).

174) Northampton County: Upper Mount Bethel Twp. $(\mathrm{n}=3)$. LJ (AMNH 169928). LLJ (AMNH 169926-27).

175) Chester County: Warwick Twp. $(\mathrm{n}=21)$. JJ (AMNH 169462-82).

176) Wyoming County: Mehoopany Twp. ( $\mathrm{n}=1$ ). JJ (AMNH 163433).

177) Wyoming County: Lemon Twp. $(\mathrm{n}=5)$. JJ (AMNH 165851-55).

178) Luzerne County: Lake Twp. $(\mathrm{n}=1)$. JJ (AMNH 163434).

179) Monroe County: Middle Smithfield Twp. $(\mathrm{n}=17)$. JJ (AMNH 169507-10, AMNH 169834-38). LJJ (AMNH 169826-32). LJJJ (AMNH 169833).

180) York County: near Dillsburg. $(\mathrm{n}=25)$. JJ $($ AMNH 160113-37).

181) Centre County: The Barrens, near Scotia. $(\mathrm{n}=16)$. JJ (AMNH 160138, AMNH 163390-404).

182) Perry County: Tuscarora State Forest. $(\mathrm{n}=26)$. JJ (AMNH 163405-30).

183) Franklin County: South Mountain. $(\mathrm{n}=16)$. JJ (AMNH 163436-51).

184) Cumberland County: Cooke Twp. $(\mathrm{n}=2)$. JJ (AMNH 163431-32).

185) Erie County: Union Twp., South Branch French Creek (n = 18). JJ (AMNH 160139-43, AMNH 167080-92).

186) Erie County: Amity Twp., Titus Bog. (n = 18). JJ (AMNH 160144-57, AMNH 167093-96). 


\section{APPENDIX 1}

(Continued)

187) Erie County: Millcreek Twp. $(\mathrm{n}=12)$. JJ (AMNH 163452-56, AMNH 167073-79).

188) McKean County: Bradford, Glendorn. $(n=10)$. JJ (AMNH 165856-65).

189) McKean County: Eldred. (n = 65). LL (AMNH 165892-936). LLJ (AMNH 166018-37).

\section{Virginia}

190) Allegheny County: $11 \mathrm{~km}$. SW Covington. (n = 4). JJ (JPB 25772-75).

\section{EAST OF THE HUDSON RIVER \\ Connecticut}

191) Fairfield County: Danbury-Ridgefield, Wooster Mountain and Mountain Pond-Eureka Lake system (n = 9). JJ (AMNH 163457, AMNH 163459-60). LJJ (AMNH 162936-37, AMNH 162940-41, AMNH 163458, AMNH 169839).

192) Litchfield County: Washington, 0.6 mi. W Carmel Hill (n = 4). JJ (AMNH 160158). LJ (AMNH 169767). LJJ (AMNH 169768). LJJJ (AMNH 160377).

193) Litchfield County: Norfolk, west and north slopes of Bald Mountain, 1100-1450 feet. (n = 3). LJJ (AMNH 169769-71).

194) Litchfield County: Woodbury, Rag Land. (n = 9). JJ (AMNH 165866). LJJ (AMNH 165972-78). LJJJ (AMNH 166038).

195) Hartford County: Granby, 0.6 mi. NW Barndoor Hills. (n = 15). JJ (AMNH 158779-84). LJJ (AMNH 158788-96).

196) Hartford County: Simsbury, 0.2 mi. N King Phillip Mountain. (n = 3). JJ (AMNH 160159-61).

197) Hartford County: Simsbury, Talcott Mountain. $(n=1)$. LLJ (AMNH 169343).

198) Hartford County: East Granby, Marsh Pond and vicinity. (n = 13). LL (AMNH 158771, AMNH 169340-42). LLJ (AMNH 169344-45). LJJ (AMNH 158823, AMNH 169349-51). LLLJ (AMNH 169346-48).

199) Hartford County: Farmington, Shade Swamp near Rattlesnake Mountain. (n = 1). LL (AMNH 169339).

200) Hartford County: Farmington, $0.4 \mathrm{mi} \mathrm{W}$ Burnt Hill (n = 9). LJJ (AMNH 169840-48).

201) Hartford County: Wethersfield, Folly Brook. $(n=7)$. LJ (AMNH 165947-52, AMNH 169766).

202) Middlesex County: Durham, Pistapaug Mtn. (n = 3). LJJ (AMNH 165979-81).

203) New Haven County: Meriden, Cathole Mtn. (n = 17). JJ (AMNH 165867). LJJ (AMNH 165982-86, AMNH 165988-93). LJJJ (AMNH 165987, AMNH 166039-42).

204) Windham County: Plainfield and Canterbury, Quinebaug Terraces. $(\mathrm{n}=20)$. LL (AMNH 169352-54, AMNH 169850-54, AMNH 169857-68).

\section{New York}

205) Westchester County: Lewisboro. $(\mathrm{n}=1)$. JJ (AMNH 163373).

206) Dutchess County: East Fishkill. $(\mathrm{n}=2)$. LL (AMNH 169367, AMNH 169869).

207) Dutchess County: Wingdale. $(\mathrm{n}=12)$. LJJ (AMNH 169870-79. LJJJ (AMNH 169880-81).

208) Dutchess County: Dover Furnace, Camp Sharparoon. ( $=14)$. LJJ (AMNH 158797-810).

209) Dutchess County: Pawling, Swamp River below Corbin Hill. $(\mathrm{n}=1)$ ). LLJ (AMNH 160267).

210) Putnam County: Patterson, Muddy Brook at Cornwall Hill Road (Putnam County 64) crossing. (n = 5). LL (AMNH 158772-76).

211) Putnam County: Great Swamp, Patterson Environmental Park. ( $=14)$. LL (AMNH 158777-78, AMNH 159991-98). LJ (AMNH 160246-47). LLJ (AMNH 158824, AMNH 160256).

212) Columbia County: Ancram. $(\mathrm{n}=2)$. JJ (AMNH 163374-75).

213) Washington County: alongside Rte. $22 \mathrm{~N}$ of Salem, Beaver Brook drainage. $(\mathrm{n}=1)$. LJJ (AMNH 153117).

\section{Massachusetts}

214) Middlesex County: Groton, wetlands along abandoned RR grade, S of Peabody Street, E of Groton School. $(\mathrm{n}=4)$. LL (AMNH 158769). LJ (AMNH 158785-86). LLJ (AMNH 158811).

215) Middlesex County: Groton, Groton Commons. $(\mathrm{n}=12)$. LL (AMNH 158770). LJ (AMNH 158812, AMNH 158816). LLJ (AMNH 158813-15, AMNH 158817-22).

216) Berkshire County: Sheffield, Schenob Brook drainage. $(\mathrm{n}=1)$. LLJ (AMNH 153120). 


\section{APPENDIX 2}

Genotypes of Ambystoma laterale, A. jeffersonianum, and unisexual specimens ordered by site number Genotypes of the specimens are ordered by site number (see appendix 1). Specimen numbers are American Museum of Natural History (AMNH) or catalogue numbers of J. P. Bogart (JPB). The sex of the specimen is female $(\mathrm{F})$ or male $(\mathrm{M})$. ?, M? or F? are juvenile individuals where the sex was difficult to determine, and $\mathrm{L}$ is a larval sample. The letters for the electrophoretic alleles, or allozymes, for each locus refer to relative mobilities of the stained enzyme on the gel (see table 4). A single letter is given for a homozygous condition so, for example, a B would be BB in a diploid, BBB in a triploid and BBBB in a tetraploid. Data that were not obtained for an individual at an isozyme locus is signified by —. Data for "blood" are mean erythrocyte area determinations or flow cytometric (FMC) determinations for ploidy based on comparative fluorescence of blood cells from individuals against a diploid standard fluorescence (see Ramsden et al. 2006). Blood data that were not obtained for an individual is signified by —. Tables in this appendix compare individuals that have the same genomotypes:

2-1: Ambystoma laterale (LL) diploids from all sites.

2-2: Ambystoma jeffersonianum (JJ) diploids from all sites.

2-3: Ambystoma laterale - jeffersonianum (LJ) diploids from all sites.

2-4: Ambystoma (2) laterale - jeffersonianum (LLJ) triploids from all sites.

2-5: Ambystoma laterale - (2) jeffersonianum (LJJ) triploids from all sites.

2-6: Ambystoma (3) laterale - jeffersonianum (LLLJ) tetraploids from all sites.

2-7: Ambystoma laterale - (3) jeffersonianum (LJJJ) tetraploids from all sites.

2-8: Ambystoma (2) laterale - (2) jeffersonianum (LLJJ) tetraploid from site 18.

Appendix 2-1: Genotypes of diploid (2n) Ambystoma laterale specimens ordered by site number.

Locus

Site Sex AMNH Aat-1 Aat-2 Idh-1 Ldh-1 Ldh-2 Mdh-1 Mpi Pgi Pgm-1 Pgm-2 Sod-1 blood

\begin{tabular}{|c|c|c|c|c|c|c|c|c|c|c|c|c|c|c|c|}
\hline 1 & 18 & $\mathrm{~F}$ & 159999 & B & B & B & B & B & $\mathrm{D}$ & $\mathrm{C}$ & - & B & B & B & 704 \\
\hline 2 & 18 & M & 160000 & B & B & B & B & B & $\mathrm{D}$ & $\mathrm{C}$ & - & B & B & B & 651 \\
\hline 3 & 42 & $\mathrm{~F}$ & 160056 & B & B & B & B & B & $\mathrm{D}$ & 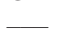 & $\mathrm{C}$ & $\mathrm{BC}$ & B & B & 515 \\
\hline 4 & 60 & M & 169855 & $\mathrm{~B}$ & B & B & B & B & $\mathrm{D}$ & $\mathrm{C}$ & $\mathrm{C}$ & B & B & B & FCM \\
\hline 5 & 60 & $\mathrm{~F}$ & 169856 & $\mathrm{~B}$ & B & B & B & B & $\mathrm{D}$ & $\mathrm{C}$ & $\mathrm{C}$ & B & B & B & FCM \\
\hline 6 & 66 & M & 169355 & B & B & B & B & A & D & $\mathrm{C}$ & $\mathrm{C}$ & B & B & B & FCM \\
\hline 7 & 66 & $\mathrm{~F}$ & 169356 & B & B & B & B & A & $\mathrm{D}$ & $\mathrm{C}$ & $\mathrm{C}$ & B & B & B & FCM \\
\hline 8 & 107 & $\mathrm{~F}$ & 169885 & $\mathrm{~B}$ & B & B & B & B & $\mathrm{D}$ & 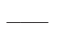 & $\mathrm{C}$ & B & B & B & $\mathrm{FCM}$ \\
\hline 9 & 107 & $\mathrm{~F}$ & 169886 & $\mathrm{~B}$ & B & B & B & A & $\mathrm{D}$ & 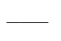 & $\mathrm{C}$ & B & B & B & $\mathrm{FCM}$ \\
\hline 10) & 107 & M & 169887 & B & B & B & B & B & $\mathrm{D}$ & $\mathrm{C}$ & $\mathrm{BC}$ & B & B & B & $\mathrm{FCM}$ \\
\hline 11 & 107 & $\mathrm{~F}$ & 169888 & $\mathrm{~B}$ & B & B & B & B & $\mathrm{D}$ & 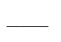 & $\mathrm{C}$ & B & B & B & FCM \\
\hline 12 & 107 & $\mathrm{~F}$ & 169889 & $\mathrm{~B}$ & B & B & B & A & $\mathrm{D}$ & - & $\mathrm{C}$ & B & B & B & FCM \\
\hline 13 & 107 & M & 169890 & B & B & B & B & B & D & 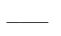 & $\mathrm{C}$ & B & B & B & FCM \\
\hline 14 & 107 & M? & 169891 & B & B & B & B & A & $\mathrm{D}$ & - & $\mathrm{C}$ & B & B & B & FCM \\
\hline 15 & 107 & M? & 169892 & $\mathrm{~B}$ & B & B & B & A & $\mathrm{D}$ & 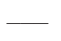 & $\mathrm{C}$ & B & B & B & FCM \\
\hline 16 & 107 & $\mathrm{~F}$ & 169893 & $\mathrm{~B}$ & B & B & B & A & $\mathrm{D}$ & - & $\mathrm{C}$ & B & B & B & $\mathrm{FCM}$ \\
\hline 17 & 108 & M & 160041 & $\mathrm{~B}$ & B & B & B & B & $\mathrm{D}$ & $\mathrm{CD}$ & 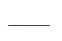 & $\mathrm{BC}$ & B & B & 757 \\
\hline 18 & 108 & $\mathrm{~F}$ & 160042 & B & B & B & B & B & $\mathrm{D}$ & $\mathrm{C}$ & $\mathrm{C}$ & B & B & B & 801 \\
\hline 19 & 108 & $\mathrm{~F}$ & 160043 & $\mathrm{~B}$ & B & B & B & B & $\mathrm{D}$ & $\mathrm{C}$ & - & B & B & B & 734 \\
\hline 20) & 108 & M & 160044 & $\mathrm{~B}$ & B & B & B & B & $\mathrm{D}$ & $\mathrm{CD}$ & - & B & B & B & 807 \\
\hline 21 & 108 & M & 160045 & B & B & B & B & B & $\mathrm{D}$ & $\mathrm{C}$ & - & B & B & B & 627 \\
\hline 22 & 108 & M & 160046 & B & B & B & B & B & $\mathrm{D}$ & $\mathrm{C}$ & 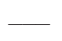 & B & B & B & 770 \\
\hline 23 & 108 & M & 160047 & $\mathrm{~B}$ & B & B & B & B & $\mathrm{D}$ & $\mathrm{CD}$ & - & B & B & B & 814 \\
\hline 24 & 108 & M & 160048 & $\mathrm{~B}$ & B & B & B & B & $\mathrm{D}$ & $\mathrm{C}$ & 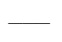 & B & B & B & 772 \\
\hline 25 & 108 & $\mathrm{~F}$ & 160049 & $\mathrm{~B}$ & B & B & B & B & $\mathrm{D}$ & $\mathrm{C}$ & - & B & B & B & 772 \\
\hline 26 & 108 & $\mathrm{~F}$ & 160050 & B & B & B & B & B & $\mathrm{D}$ & - & - & B & B & B & 732 \\
\hline 27 & 108 & M & 160051 & $\mathrm{~B}$ & B & B & B & A & $\mathrm{D}$ & $\mathrm{CD}$ & $\mathrm{C}$ & B & B & B & 788 \\
\hline 28 & 108 & M & 160052 & $\mathrm{~B}$ & B & B & B & B & $\mathrm{D}$ & - & $\mathrm{C}$ & B & B & B & 709 \\
\hline
\end{tabular}


APPENDIX 2-1

(Continued)

\begin{tabular}{|c|c|c|c|c|c|c|c|c|c|c|c|c|c|c|c|}
\hline & \multirow[b]{2}{*}{ Site } & \multirow[b]{2}{*}{ Sex } & \multirow[b]{2}{*}{ AMNH } & \multicolumn{12}{|c|}{ Locus } \\
\hline & & & & Aat-1 & Aat-2 & Idh-1 & Ldh-1 & Ldh-2 & Mdh-1 & Mpi & Pgi & Pgm-1 & Pgm-2 & Sod-1 & blood \\
\hline 29 & 108 & M & 160053 & B & B & B & B & B & $\mathrm{D}$ & - & $\mathrm{C}$ & B & B & B & 523 \\
\hline 30) & 108 & M & 160054 & B & B & B & B & A & $\mathrm{D}$ & - & 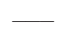 & B & B & B & 800 \\
\hline 31 & 110 & M & 169369 & B & B & B & B & B & $\mathrm{D}$ & $\mathrm{C}$ & - & B & $\mathrm{AB}$ & B & FCM \\
\hline 32 & 110 & M & 169370 & B & B & B & B & B & $\mathrm{D}$ & $\mathrm{C}$ & - & B & B & B & FCM \\
\hline 33 & 110 & $\mathrm{~F}$ & 169371 & B & B & B & B & B & $\mathrm{D}$ & $\mathrm{C}$ & $\mathrm{C}$ & B & B & B & FCM \\
\hline 34 & 110 & M & 169372 & B & B & B & B & B & $\mathrm{D}$ & $\mathrm{C}$ & $\mathrm{C}$ & B & $\mathrm{AB}$ & B & FCM \\
\hline 35 & 110 & $\mathrm{~F}$ & 169373 & B & B & B & B & B & $\mathrm{D}$ & $\mathrm{C}$ & $\mathrm{C}$ & B & $\mathrm{AB}$ & B & FCM \\
\hline 36 & 110 & M & 169374 & B & B & B & B & B & $\mathrm{D}$ & $\mathrm{C}$ & $\mathrm{C}$ & B & $\mathrm{AB}$ & B & FCM \\
\hline 37 & 110 & $\mathrm{~F}$ & 169375 & B & B & B & B & B & $\mathrm{D}$ & $\mathrm{C}$ & $\mathrm{C}$ & B & $\mathrm{AB}$ & B & FCM \\
\hline 38 & 110 & $\mathrm{~F}$ & 169376 & B & B & B & B & B & $\mathrm{D}$ & $\mathrm{C}$ & $\mathrm{C}$ & B & B & B & FCM \\
\hline 39 & 110 & $\mathrm{~F}$ & 169377 & B & B & B & B & B & $\mathrm{D}$ & $\mathrm{C}$ & $\mathrm{C}$ & B & B & B & FCM \\
\hline 40) & 110 & $\mathrm{~F}$ & 169378 & B & B & B & B & B & $\mathrm{D}$ & $\mathrm{C}$ & $\mathrm{C}$ & B & $\mathrm{AB}$ & B & FCM \\
\hline 41 & 110 & $\mathrm{~F}$ & 169379 & B & B & B & B & B & $\mathrm{D}$ & $\mathrm{C}$ & $\mathrm{C}$ & B & $\mathrm{AB}$ & B & FCM \\
\hline 42 & 110 & M & 169380 & B & B & B & B & B & $\mathrm{D}$ & $\mathrm{C}$ & $\mathrm{C}$ & B & B & B & FCM \\
\hline 43 & 110 & $\mathrm{~F}$ & 169381 & B & B & B & B & B & $\mathrm{D}$ & $\mathrm{C}$ & $\mathrm{C}$ & B & B & B & FCM \\
\hline 44 & 110 & $\mathrm{~F}$ & 169382 & B & B & B & B & B & $\mathrm{D}$ & $\mathrm{C}$ & $\mathrm{C}$ & B & B & B & FCM \\
\hline 45 & 110 & $\mathrm{~F}$ & 169383 & B & B & B & B & B & $\mathrm{D}$ & $\mathrm{C}$ & $\mathrm{C}$ & B & $\mathrm{AB}$ & B & FCM \\
\hline 46 & 110 & M & 169384 & B & B & B & B & B & $\mathrm{D}$ & $\mathrm{C}$ & $\mathrm{C}$ & B & B & B & FCM \\
\hline 47 & 110 & $\mathrm{~F}$ & 169882 & B & B & B & B & B & $\mathrm{D}$ & $\mathrm{C}$ & $\mathrm{C}$ & B & B & B & FCM \\
\hline 48 & 110 & $\mathrm{~F}$ & 169883 & B & B & B & B & B & $\mathrm{D}$ & $\mathrm{C}$ & $\mathrm{C}$ & B & $\mathrm{AB}$ & B & FCM \\
\hline 49 & 110 & $\mathrm{~F}$ & 169884 & B & B & B & B & B & $\mathrm{D}$ & $\mathrm{C}$ & $\mathrm{C}$ & B & $\mathrm{AB}$ & B & FCM \\
\hline 50) & 111 & $\mathrm{~F}$ & 169386 & B & B & B & B & A & $\mathrm{D}$ & $\mathrm{C}$ & 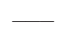 & B & B & B & FCM \\
\hline 51 & 111 & $\mathrm{~F}$ & 169387 & B & B & B & $\mathrm{B}$ & A & $\mathrm{D}$ & $\mathrm{C}$ & $\mathrm{C}$ & B & B & B & FCM \\
\hline 52 & 111 & $\mathrm{~F}$ & 169388 & $\mathrm{~B}$ & B & B & B & A & $\mathrm{D}$ & $\mathrm{C}$ & 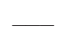 & B & B & B & FCM \\
\hline 53 & 111 & M & 169389 & B & B & B & B & A & $\mathrm{D}$ & 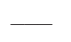 & $\mathrm{C}$ & B & B & B & FCM \\
\hline 54 & 111 & $\mathrm{~F}$ & 169390 & B & B & B & B & A & $\mathrm{D}$ & $\mathrm{C}$ & 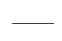 & B & B & B & FCM \\
\hline 55 & 111 & $\mathrm{~F}$ & 169391 & B & B & B & B & A & $\mathrm{D}$ & $\mathrm{C}$ & 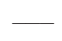 & B & B & B & FCM \\
\hline 56 & 111 & M & 169392 & B & B & B & B & B & $\mathrm{D}$ & $\mathrm{C}$ & $\mathrm{C}$ & B & B & B & FCM \\
\hline 57 & 111 & M & 169393 & B & B & B & B & A & $\mathrm{D}$ & $\mathrm{C}$ & $\mathrm{C}$ & B & B & B & FCM \\
\hline 58 & 111 & $\mathrm{~F}$ & 169394 & B & B & B & B & B & $\mathrm{D}$ & $\mathrm{C}$ & $\mathrm{C}$ & B & B & B & FCM \\
\hline 59 & 113 & M & 162962 & B & B & B & B & B & $\mathrm{D}$ & $\mathrm{C}$ & 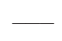 & B & B & B & 691 \\
\hline 60) & 113 & $\mathrm{~F}$ & 162963 & B & B & B & $\mathrm{B}$ & B & $\mathrm{D}$ & $\mathrm{C}$ & $\mathrm{C}$ & B & $\mathrm{BC}$ & B & - \\
\hline 61 & 113 & M & 162964 & B & B & B & B & B & $\mathrm{D}$ & $\mathrm{C}$ & $\mathrm{C}$ & B & $\mathrm{B}$ & B & 757 \\
\hline 62 & 113 & M & 162965 & B & B & QC & A & B & $\mathrm{D}$ & $\mathrm{C}$ & 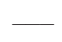 & B & B & B & 913 \\
\hline 63 & 113 & M & 164587 & B & B & B & B & B & $\mathrm{D}$ & $\mathrm{C}$ & $\mathrm{C}$ & B & B & B & 723 \\
\hline 64 & 113 & $\mathrm{~F}$ & 164588 & B & B & B & B & B & $\mathrm{D}$ & $\mathrm{C}$ & $\mathrm{C}$ & B & B & B & 734 \\
\hline 65 & 127 & M & 160001 & B & B & 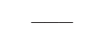 & 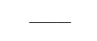 & - & 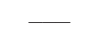 & $\mathrm{C}$ & 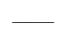 & B & B & B & - \\
\hline 66 & 127 & $\mathrm{~F}$ & 160002 & B & B & B & B & B & $\mathrm{D}$ & $\mathrm{C}$ & $\mathrm{C}$ & B & B & B & 794 \\
\hline 67 & 127 & $\mathrm{~F}$ & 160003 & B & B & B & B & $\mathrm{AB}$ & $\mathrm{D}$ & $\mathrm{C}$ & $\mathrm{C}$ & B & B & B & 700 \\
\hline 68 & 127 & M & 160004 & B & B & B & B & $\mathrm{A}$ & $\mathrm{D}$ & $\mathrm{C}$ & $\mathrm{C}$ & B & B & B & 705 \\
\hline 69 & 127 & M & 160005 & B & B & B & B & A & $\mathrm{D}$ & $\mathrm{C}$ & $\mathrm{C}$ & B & B & B & 671 \\
\hline 70) & 127 & M & 160006 & B & B & B & B & B & $\mathrm{D}$ & $\mathrm{C}$ & 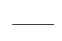 & B & B & B & 591 \\
\hline 71 & 127 & $\mathrm{~F}$ & 160007 & B & B & B & B & A & $\mathrm{D}$ & $\mathrm{C}$ & $\mathrm{C}$ & B & B & B & 690 \\
\hline 72 & 127 & M & 160008 & B & B & B & B & B & $\mathrm{D}$ & - & - & B & B & B & 787 \\
\hline 73 & 127 & M & 160009 & B & B & B & B & B & $\mathrm{D}$ & - & 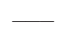 & B & B & B & 739 \\
\hline 74 & 127 & $\mathrm{~F}$ & 160010 & B & B & B & B & $\mathrm{AB}$ & $\mathrm{D}$ & $\mathrm{C}$ & $\mathrm{C}$ & B & B & B & 774 \\
\hline 75 & 127 & M & 160011 & B & B & B & B & B & $\mathrm{D}$ & $\mathrm{C}$ & - & B & B & B & 620 \\
\hline 76 & 127 & $\mathrm{~F}$ & 160012 & B & B & B & B & B & $\mathrm{D}$ & $\mathrm{C}$ & - & $\mathrm{B}$ & B & B & 646 \\
\hline 77 & 127 & $\mathrm{~F}$ & 160013 & B & B & B & B & $\mathrm{AB}$ & $\mathrm{D}$ & $\mathrm{C}$ & $\mathrm{C}$ & B & B & B & 725 \\
\hline 78 & 127 & M & 160014 & B & B & B & B & A & $\mathrm{D}$ & $\mathrm{C}$ & $\mathrm{C}$ & B & B & B & 682 \\
\hline 79 & 127 & $\mathrm{~F}$ & 160015 & $\mathrm{~B}$ & B & B & $\mathrm{BD}$ & B & $\mathrm{D}$ & $\mathrm{C}$ & - & B & B & B & 714 \\
\hline 80) & 127 & M & 160016 & B & B & B & B & A & $\mathrm{D}$ & $\mathrm{C}$ & $\mathrm{C}$ & B & B & B & 740 \\
\hline
\end{tabular}


APPENDIX 2-1

(Continued)

Locus

Site Sex AMNH Aat-1 Aat-2 Idh-1 Ldh-1 Ldh-2 Mdh-1 Mpi Pgi Pgm-1 Pgm-2 Sod-1 blood

\begin{tabular}{|c|c|c|c|c|c|c|c|c|c|c|c|c|c|c|c|}
\hline 81 & 127 & $\mathrm{M}$ & 160017 & $\mathrm{~B}$ & $\mathrm{~B}$ & B & B & A & $\mathrm{D}$ & $\mathrm{C}$ & $\mathrm{C}$ & B & B & B & 785 \\
\hline 82 & 127 & $\mathrm{M}$ & 160018 & $\mathrm{~B}$ & $\mathrm{~B}$ & B & B & $\mathrm{B}$ & $\mathrm{D}$ & $\mathrm{C}$ & $\mathrm{C}$ & B & B & B & 692 \\
\hline 83 & 127 & $\mathrm{~F}$ & 160019 & B & B & B & B & B & $\mathrm{D}$ & $\mathrm{C}$ & - & B & B & B & 828 \\
\hline 84 & 127 & $\mathrm{~F}$ & 160020 & B & B & B & B & $\mathrm{AB}$ & $\mathrm{D}$ & $\mathrm{C}$ & $\mathrm{C}$ & B & B & B & 740 \\
\hline 85 & 127 & $\mathrm{M}$ & 160021 & B & B & B & B & A & $\mathrm{D}$ & $\mathrm{C}$ & $\mathrm{C}$ & B & B & B & 778 \\
\hline 86 & 127 & $\mathrm{M}$ & 160022 & B & B & B & $\mathrm{D}$ & B & $\mathrm{D}$ & - & - & B & B & B & 608 \\
\hline 87 & 127 & $\mathrm{M}$ & 160023 & $\mathrm{~B}$ & $\mathrm{~B}$ & B & B & B & $\mathrm{D}$ & $\mathrm{C}$ & - & B & B & B & 715 \\
\hline 88 & 127 & $\mathrm{~F}$ & 160024 & $\mathrm{~B}$ & B & B & B & B & D & $\mathrm{C}$ & $\mathrm{C}$ & B & B & B & 695 \\
\hline 89 & 127 & $\mathrm{M}$ & 160025 & B & B & B & B & $\mathrm{AB}$ & $\mathrm{D}$ & $\mathrm{C}$ & $\mathrm{C}$ & B & B & B & 762 \\
\hline 90) & 127 & $\mathrm{M}$ & 160026 & $\mathrm{~B}$ & $\mathrm{~B}$ & B & B & $\mathrm{AB}$ & $\mathrm{D}$ & $\mathrm{C}$ & $\mathrm{C}$ & B & B & B & 661 \\
\hline 91 & 127 & $\mathrm{~F}$ & 160027 & B & B & B & B & $\mathrm{AB}$ & $\mathrm{D}$ & $\mathrm{C}$ & $\mathrm{C}$ & B & B & B & 657 \\
\hline 92 & 127 & $\mathrm{~F}$ & 160028 & B & B & B & BD & B & D & - & - & B & B & B & 756 \\
\hline 93 & 127 & M & 160029 & B & B & B & - & B & $\mathrm{D}$ & - & - & B & B & B & 746 \\
\hline 94 & 127 & $\mathrm{M}$ & 160030 & $\mathrm{~B}$ & $\mathrm{~B}$ & B & B & B & $\mathrm{D}$ & $\mathrm{C}$ & - & B & B & B & 726 \\
\hline 95 & 127 & $\mathrm{~F}$ & 160031 & $\mathrm{~B}$ & $\mathrm{~B}$ & B & B & B & $\mathrm{D}$ & $\mathrm{C}$ & $\mathrm{C}$ & B & B & B & 800 \\
\hline 96 & 127 & $\mathrm{M}$ & 160032 & B & B & B & B & A & $\mathrm{D}$ & 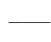 & - & B & B & B & 687 \\
\hline 97 & 127 & $\mathrm{~F}$ & 160033 & $\mathrm{~B}$ & $\mathrm{~B}$ & B & - & B & $\mathrm{D}$ & 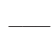 & 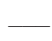 & A & B & B & 669 \\
\hline 98 & 127 & $\mathrm{~F}$ & 160034 & $\mathrm{~B}$ & B & B & B & B & $\mathrm{D}$ & 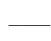 & - & A & B & B & 735 \\
\hline 99 & 127 & $\mathrm{~F}$ & 160035 & B & B & B & B & B & D & 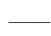 & - & B & B & B & 710 \\
\hline 100) & 127 & $\mathrm{M}$ & 160036 & B & $\mathrm{B}$ & B & $\mathrm{BD}$ & B & $\mathrm{D}$ & 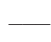 & - & B & B & B & 669 \\
\hline 101 & 127 & $\mathrm{M}$ & 160037 & $\mathrm{~B}$ & B & B & $\mathrm{BD}$ & B & $\mathrm{D}$ & $\mathrm{C}$ & - & B & B & B & 640 \\
\hline 102 & 127 & $\mathrm{~F}$ & 160038 & B & $\mathrm{B}$ & B & BD & $\mathrm{BC}$ & $\mathrm{D}$ & 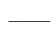 & - & B & B & B & 789 \\
\hline 103 & 127 & $\mathrm{~F}$ & 160039 & $\mathrm{~B}$ & B & B & B & $\mathrm{AB}$ & $\mathrm{D}$ & 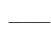 & - & A & B & B & 679 \\
\hline 104 & 127 & $\mathrm{M}$ & 160040 & B & B & B & B & B & $\mathrm{D}$ & 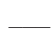 & - & B & B & B & 695 \\
\hline 105 & 127 & $\mathrm{M}$ & 162968 & B & B & B & $\mathrm{D}$ & $\mathrm{AC}$ & $\mathrm{D}$ & 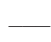 & B & B & B & 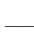 & 949 \\
\hline 106 & 129 & $\mathrm{~F}$ & 162969 & $\mathrm{~B}$ & $\mathrm{~B}$ & B & B & B & $\mathrm{D}$ & $\mathrm{C}$ & 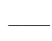 & B & B & B & 787 \\
\hline 107 & 129 & $\mathrm{M}$ & 162970 & $\mathrm{~B}$ & B & B & B & B & $\mathrm{D}$ & $\mathrm{C}$ & - & B & B & B & 750 \\
\hline 108 & 129 & $\mathrm{~F}$ & 165869 & B & B & B & B & B & $\mathrm{D}$ & $\mathrm{C}$ & - & B & B & B & 704 \\
\hline 109 & 129 & $\mathrm{~F}$ & 165870 & B & B & B & B & B & $\mathrm{D}$ & $\mathrm{C}$ & - & B & B & B & 708 \\
\hline 110) & 129 & $\mathrm{~F}$ & 165871 & $\mathrm{~B}$ & B & B & $\mathrm{BD}$ & B & $\mathrm{D}$ & 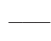 & - & B & $\mathrm{AB}$ & B & 688 \\
\hline 111 & 129 & $\mathrm{~F}$ & 165872 & B & B & B & $\mathrm{BD}$ & B & $\mathrm{D}$ & $\mathrm{C}$ & - & B & B & B & 695 \\
\hline 112 & 129 & $\mathrm{~F}$ & 165873 & B & B & B & B & B & $\mathrm{D}$ & $\mathrm{C}$ & - & B & B & B & 697 \\
\hline 113 & 129 & $\mathrm{M}$ & 165874 & B & B & B & B & B & $\mathrm{D}$ & $\mathrm{C}$ & 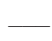 & B & B & B & 744 \\
\hline 114 & 132 & $\mathrm{~F}$ & 162966 & $\mathrm{~B}$ & $\mathrm{~B}$ & B & B & B & $\mathrm{D}$ & 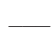 & 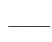 & $\mathrm{B}$ & B & B & 834 \\
\hline 115 & 133 & $\mathrm{~F}$ & 165875 & B & B & B & B & B & $\mathrm{D}$ & $\mathrm{C}$ & - & B & B & B & 598 \\
\hline 116 & 133 & $\mathrm{~F}$ & 165876 & B & B & B & $\mathrm{D}$ & B & $\mathrm{D}$ & $\mathrm{C}$ & - & B & B & B & 628 \\
\hline 117 & 133 & M & 165877 & B & B & B & B & B & $\mathrm{D}$ & $\mathrm{C}$ & - & B & B & B & 717 \\
\hline 118 & 134 & $\mathrm{~F}$ & 162967 & $\mathrm{~B}$ & $\mathrm{~B}$ & B & $\mathrm{BD}$ & B & $\mathrm{D}$ & 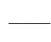 & - & B & B & B & 876 \\
\hline 119 & 136 & $\mathrm{~F}$ & 165878 & $\mathrm{~B}$ & B & B & B & A & $\mathrm{D}$ & $\mathrm{C}$ & - & $\mathrm{B}$ & B & B & 643 \\
\hline 120) & 136 & $\mathrm{M}$ & 165879 & B & B & B & $\mathrm{BD}$ & $\mathrm{B}$ & D & $\mathrm{C}$ & - & B & B & B & 558 \\
\hline 121 & 136 & $\mathrm{M}$ & 165880 & $\mathrm{~B}$ & $\mathrm{~B}$ & B & B & B & $\mathrm{D}$ & $\mathrm{C}$ & $\mathrm{C}$ & B & B & B & 580 \\
\hline 122 & 136 & $\mathrm{~F}$ & 165881 & $\mathrm{~B}$ & $\mathrm{~B}$ & B & B & B & $\mathrm{D}$ & $\mathrm{C}$ & - & B & B & B & 593 \\
\hline 123 & 136 & M & 165882 & B & B & B & $\mathrm{BD}$ & B & $\mathrm{D}$ & $\mathrm{C}$ & $\mathrm{C}$ & B & B & B & 653 \\
\hline 124 & 136 & $\mathrm{M}$ & 165883 & B & B & B & B & B & $\mathrm{D}$ & 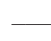 & - & B & B & B & 779 \\
\hline 125 & 136 & $\mathrm{~F}$ & 165884 & $\mathrm{~B}$ & $\mathrm{~B}$ & B & B & B & $\mathrm{D}$ & $\mathrm{C}$ & $\mathrm{C}$ & B & B & B & 648 \\
\hline 126 & 143 & $\mathrm{M}$ & 162971 & $\mathrm{~B}$ & $\mathrm{~B}$ & B & B & B & $\mathrm{D}$ & $\mathrm{C}$ & $\mathrm{C}$ & B & B & B & 874 \\
\hline 127 & 143 & $\mathrm{~F}$ & 167021 & B & B & B & B & A & $\mathrm{D}$ & $\mathrm{C}$ & $\mathrm{C}$ & B & B & B & 781 \\
\hline 128 & 143 & $\mathrm{~F}$ & 167022 & B & B & B & B & B & $\mathrm{D}$ & $\mathrm{C}$ & $\mathrm{C}$ & B & B & B & 770 \\
\hline 129 & 143 & $\mathrm{~F}$ & 167023 & $\mathrm{~B}$ & $\mathrm{~B}$ & B & B & B & $\mathrm{D}$ & $\mathrm{C}$ & $\mathrm{C}$ & B & B & B & 772 \\
\hline 130) & 143 & M & 167024 & B & $\mathrm{B}$ & B & B & B & $\mathrm{D}$ & $\mathrm{C}$ & $\mathrm{C}$ & B & B & B & 814 \\
\hline 131 & 143 & $\mathrm{M}$ & 167025 & B & B & B & B & A & $\mathrm{D}$ & $\mathrm{C}$ & $\mathrm{C}$ & B & B & B & 828 \\
\hline 132 & 143 & M & 167026 & B & B & B & BD & B & D & $\mathrm{C}$ & $\mathrm{C}$ & B & B & B & 718 \\
\hline
\end{tabular}


APPENDIX 2-1

(Continued)

\begin{tabular}{|c|c|c|c|c|c|c|c|c|c|c|c|c|c|c|c|}
\hline & \multirow[b]{2}{*}{ Site } & \multirow[b]{2}{*}{ Sex } & \multirow[b]{2}{*}{ AMNH } & \multicolumn{12}{|c|}{ Locus } \\
\hline & & & & Aat-1 & Aat-2 & Idh-1 & Ldh-1 & Ldh-2 & Mdh-1 & Mpi & Pgi & Pgm-1 & Pgm-2 & Sod-1 & blood \\
\hline 133 & 143 & M & 167027 & B & B & B & B & B & $\mathrm{D}$ & $\mathrm{C}$ & $\mathrm{C}$ & B & B & B & 764 \\
\hline 134 & 143 & M & 167028 & B & B & B & B & B & $\mathrm{D}$ & $\mathrm{C}$ & $\mathrm{C}$ & B & B & B & 773 \\
\hline 135 & 143 & M & 167029 & B & B & B & B & B & $\mathrm{D}$ & $\mathrm{C}$ & $\mathrm{C}$ & B & B & B & 793 \\
\hline 136 & 143 & $\mathrm{M}$ & 167030 & B & B & B & B & B & D & $\mathrm{C}$ & C & B & B & B & 811 \\
\hline 137 & 143 & $\mathrm{~F}$ & 167031 & B & B & B & BD & B & D & C & $\mathrm{C}$ & B & B & B & 782 \\
\hline 138 & 143 & $\mathrm{~F}$ & 167032 & B & B & B & $\mathrm{BD}$ & B & D & $\mathrm{C}$ & C & B & B & B & 740 \\
\hline 139 & 144 & M & 165885 & B & B & B & B & B & D & 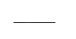 & C & B & B & B & 653 \\
\hline 140) & 144 & $\mathrm{~F}$ & 165886 & B & B & B & B & B & D & C & C & BC & B & B & 728 \\
\hline 141 & 144 & M & 165887 & B & B & B & B & B & D & C & - & B & B & B & 700 \\
\hline 142 & 144 & $\mathrm{~F}$ & 165888 & B & B & B & B & B & D & C & - & B & B & B & 687 \\
\hline 143 & 144 & $\mathrm{M}$ & 165889 & B & B & B & B & B & $\mathrm{D}$ & $\mathrm{C}$ & - & B & B & B & 686 \\
\hline 144 & 144 & $\mathrm{~F}$ & 165890 & B & B & B & B & B & $\mathrm{D}$ & $\mathrm{C}$ & - & B & B & B & 696 \\
\hline 145 & 144 & M & 165891 & B & B & B & B & B & D & $\mathrm{C}$ & - & B & B & B & 802 \\
\hline 146 & 145 & $\mathrm{M}$ & 167037 & B & B & B & B & A & $\mathrm{D}$ & $\mathrm{C}$ & $\mathrm{C}$ & B & B & B & 733 \\
\hline 147 & 145 & $\mathrm{~F}$ & 167038 & B & B & B & B & B & D & C & C & B & B & B & 705 \\
\hline 148 & 145 & $\mathrm{~F}$ & 167039 & B & B & B & $\mathrm{BD}$ & A & D & C & C & B & B & B & 640 \\
\hline 149 & 145 & $\mathrm{M}$ & 167040 & B & B & B & B & B & D & $\mathrm{C}$ & $\mathrm{C}$ & B & B & B & 745 \\
\hline 150) & 146 & M & 167056 & B & B & B & B & B & D & C & - & B & B & B & FCM \\
\hline 151 & 147 & $\mathrm{M}$ & 167057 & B & B & B & B & B & D & $\mathrm{C}$ & - & B & B & B & FCM \\
\hline 152 & 147 & $\mathrm{M}$ & 167058 & B & B & B & B & B & D & $\mathrm{C}$ & - & B & B & B & 783 \\
\hline 153 & 147 & $\mathrm{~F}$ & 167059 & B & B & B & B & B & D & C & - & B & B & B & 762 \\
\hline 154 & 147 & $\mathrm{M}$ & 167060 & B & B & B & B & B & D & C & - & B & B & B & 885 \\
\hline 155 & 147 & M & 167061 & B & B & B & B & B & $\mathrm{D}$ & $\mathrm{C}$ & - & B & B & B & 794 \\
\hline 156 & 147 & M & 167062 & B & B & B & B & B & $\mathrm{D}$ & $\mathrm{C}$ & - & B & B & B & 879 \\
\hline 157 & 147 & $\mathrm{M}$ & 167063 & B & B & B & B & B & D & C & - & B & B & B & 745 \\
\hline 158 & 150 & M & 169414 & B & B & B & B & A & $\mathrm{CD}$ & C & $\mathrm{AC}$ & B & B & B & FCM \\
\hline 159 & 150 & $\mathrm{~F}$ & 169415 & B & B & B & B & B & D & C & - & B & B & B & FCM \\
\hline 160) & 150 & $\mathrm{~F}$ & 169416 & B & B & B & B & A & $\mathrm{D}$ & $\mathrm{C}$ & - & B & B & B & FCM \\
\hline 161 & 150 & $\mathrm{~F}$ & 169417 & B & B & B & B & B & CD & C & $\mathrm{AC}$ & B & B & B & FCM \\
\hline 162 & 150 & $\mathrm{~F}$ & 169418 & B & B & B & B & A & D & C & - & B & B & B & FCM \\
\hline 163 & 150 & $\mathrm{~F}$ & 169419 & B & B & B & B & A & D & C & - & B & B & B & FCM \\
\hline 164 & 150 & M & 169420 & B & B & B & B & A & D & C & C & B & B & B & FCM \\
\hline 165 & 150 & $\mathrm{~F}$ & 169421 & B & B & B & B & B & D & $\mathrm{C}$ & C & B & B & B & FCM \\
\hline 166 & 150 & $\mathrm{~F}$ & 169422 & B & B & B & B & B & D & CD & - & B & B & B & FCM \\
\hline 167 & 150 & $\mathrm{~F}$ & 169423 & B & B & B & B & A & D & C & - & B & B & B & FCM \\
\hline 168 & 150 & $\mathrm{~F}$ & 169424 & B & B & B & B & B & D & C & C & B & B & B & FCM \\
\hline 169 & 150 & M & 169425 & B & B & B & B & A & D & C & - & B & B & B & FCM \\
\hline 170) & 150 & $\mathrm{~F}$ & 169426 & B & B & B & B & A & D & C & - & B & B & B & FCM \\
\hline 171 & 150 & $\mathrm{~F}$ & 169427 & B & B & B & B & B & D & C & - & B & B & B & FCM \\
\hline 172 & 150 & $\mathrm{~F}$ & 169428 & B & B & B & B & $\mathrm{AB}$ & D & C & - & $\mathrm{AB}$ & B & B & FCM \\
\hline 173 & 150 & M & 169429 & B & B & B & B & B & $\mathrm{D}$ & $\mathrm{C}$ & $\mathrm{C}$ & B & B & B & FCM \\
\hline 174 & 150 & $\mathrm{~F}$ & 169430 & B & B & B & B & B & D & C & C & $\mathrm{AB}$ & B & B & FCM \\
\hline 175 & 151 & M & 169431 & B & B & B & B & B & D & C & C & B & B & B & FCM \\
\hline 176 & 151 & $\mathrm{~F}$ & 169432 & B & B & B & B & A & D & $\mathrm{C}$ & C & B & B & B & FCM \\
\hline 177 & 151 & M & 169433 & B & B & B & B & B & D & C & C & B & B & B & FCM \\
\hline 178 & 151 & M & 169434 & B & B & B & B & B & D & C & C & B & B & B & FCM \\
\hline 179 & 151 & $\mathrm{M}$ & 169435 & B & B & B & B & A & D & $\mathrm{C}$ & - & B & B & B & FCM \\
\hline 180) & 151 & $\mathrm{~F}$ & 169436 & B & B & B & B & A & D & C & C & B & B & B & FCM \\
\hline 181 & 151 & $\mathrm{~F}$ & 169437 & B & B & B & B & A & D & C & 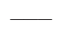 & $\mathrm{AB}$ & B & B & FCM \\
\hline 182 & 152 & $\mathrm{~F}$ & 169438 & B & B & B & B & B & $\mathrm{D}$ & $\mathrm{C}$ & C & B & B & B & FCM \\
\hline 183 & 152 & $\mathrm{~F}$ & 169439 & B & B & B & B & $\mathrm{AB}$ & D & C & C & B & B & B & FCM \\
\hline 184 & 152 & M & 169440 & B & B & B & B & B & $\mathrm{D}$ & $\mathrm{C}$ & $\mathrm{C}$ & B & B & B & FCM \\
\hline
\end{tabular}


APPENDIX 2-1

(Continued)

Locus

Site Sex AMNH Aat-1 Aat-2 Idh-1 Ldh-1 Ldh-2 Mdh-1 Mpi Pgi Pgm-1 Pgm-2 Sod-1 blood

\begin{tabular}{|c|c|c|c|c|c|c|c|c|c|c|c|c|c|c|c|}
\hline 185 & 152 & $\mathrm{~F}$ & 169441 & B & B & B & B & A & D & $\mathrm{C}$ & $\mathrm{C}$ & B & B & B & FCM \\
\hline 186 & 152 & M & 169442 & B & B & B & B & B & D & $\mathrm{C}$ & $\mathrm{C}$ & B & B & B & FCM \\
\hline 187 & 152 & M & 169443 & B & B & B & B & A & D & - & - & - & B & B & FCM \\
\hline 188 & 152 & F & 169444 & B & B & B & B & A & D & $\mathrm{C}$ & - & B & B & B & FCM \\
\hline 189 & 152 & M & 169445 & B & B & B & B & A & D & $\mathrm{C}$ & - & B & B & B & FCM \\
\hline 190) & 153 & F & 169368 & B & B & B & B & A & - & - & - & B & B & 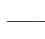 & - \\
\hline 191 & 154 & M & 160057 & B & B & B & B & A & D & $\mathrm{C}$ & - & B & B & B & 699 \\
\hline 192 & 154 & M & 160058 & B & B & B & B & B & D & $\mathrm{C}$ & - & B & B & B & 671 \\
\hline 193 & 154 & M & 160059 & B & B & B & B & A & D & $\mathrm{C}$ & - & B & B & B & 642 \\
\hline 194 & 154 & M & 160060 & B & B & B & B & B & D & $\mathrm{C}$ & - & B & B & B & 656 \\
\hline 195 & 154 & M & 160061 & B & B & B & B & B & D & $\mathrm{C}$ & - & B & B & B & 780 \\
\hline 196 & 154 & M & 160062 & B & B & B & B & B & D & $\mathrm{C}$ & - & B & B & B & 691 \\
\hline 197 & 154 & $\mathrm{~F}$ & 160063 & B & B & B & B & $\mathrm{AB}$ & D & $\mathrm{C}$ & C & B & B & B & 621 \\
\hline 198 & 154 & $\mathrm{~F}$ & 160064 & B & B & B & B & B & D & $\mathrm{C}$ & C & B & B & B & 657 \\
\hline 199 & 154 & F & 160065 & B & B & B & B & B & D & $\mathrm{C}$ & C & B & B & B & 666 \\
\hline 200) & 154 & F & 160066 & B & B & B & B & B & D & $\mathrm{C}$ & C & B & B & B & 664 \\
\hline 201 & 154 & M & 160067 & B & B & B & B & B & D & $\mathrm{C}$ & C & B & B & B & 686 \\
\hline 202 & 154 & $\mathrm{~F}$ & 160068 & B & B & B & B & B & D & $\mathrm{C}$ & $\mathrm{C}$ & B & B & B & 596 \\
\hline 203 & 154 & M & 160071 & B & B & B & B & B & D & $\mathrm{C}$ & - & B & B & B & 641 \\
\hline 204 & 155 & F & 160069 & B & B & B & B & A & D & $\mathrm{C}$ & 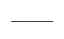 & B & B & B & 618 \\
\hline 205 & 155 & M & 160070 & B & B & B & B & A & D & $\mathrm{C}$ & C & B & B & B & 698 \\
\hline 206 & 162 & M & 166988 & B & B & B & B & B & D & $\mathrm{C}$ & C & $\mathrm{BC}$ & B & B & 739 \\
\hline 207 & 162 & F & 166989 & B & B & B & B & B & D & $\mathrm{C}$ & C & B & B & B & 802 \\
\hline 208 & 162 & M & 166990 & B & B & B & B & B & D & $\mathrm{C}$ & - & B & B & B & FCM \\
\hline 209 & 162 & M & 169357 & B & B & B & B & B & D & $\mathrm{C}$ & C & B & B & B & FCM \\
\hline 210) & 162 & M & 169358 & B & B & B & B & B & D & $\mathrm{C}$ & C & B & B & B & FCM \\
\hline 211 & 168 & $\mathrm{~F}$ & 160072 & B & B & B & B & A & D & $\mathrm{C}$ & $\mathrm{C}$ & B & B & B & 790 \\
\hline 212 & 168 & M & 160073 & B & B & B & B & A & D & $\mathrm{C}$ & 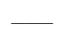 & B & B & B & 653 \\
\hline 213 & 168 & F & 160074 & B & B & B & B & A & D & - & C & B & B & B & 572 \\
\hline 214 & 168 & M & 160075 & B & B & B & B & A & D & - & - & B & B & B & 742 \\
\hline 215 & 168 & F & 160076 & B & B & B & B & A & D & $\mathrm{C}$ & C & B & B & B & 680 \\
\hline 216 & 168 & F & 160077 & B & B & B & B & B & D & - & C & B & B & B & 698 \\
\hline 217 & 168 & M & 160078 & B & B & B & B & A & D & $\mathrm{C}$ & - & B & B & B & 769 \\
\hline 218 & 168 & F & 160079 & B & B & B & B & A & D & $\mathrm{C}$ & - & B & B & B & 779 \\
\hline 219 & 168 & M & 169925 & B & B & B & B & B & D & $\mathrm{C}$ & $\mathrm{AC}$ & B & B & B & FCM \\
\hline 220) & 189 & M & 165892 & B & B & B & BD & B & D & $\mathrm{C}$ & C & B & B & B & 610 \\
\hline 221 & 189 & M & 165893 & B & B & B & D & B & D & $\mathrm{C}$ & C & B & B & B & 693 \\
\hline 222 & 189 & M & 165894 & B & B & B & BD & A & D & $\mathrm{C}$ & C & B & B & B & 690 \\
\hline 223 & 189 & $\mathrm{~F}$ & 165895 & B & B & B & BD & B & D & $\mathrm{C}$ & C & B & B & B & 813 \\
\hline 224 & 189 & $\mathrm{~F}$ & 165896 & B & B & B & BD & A & D & $\mathrm{C}$ & C & C & B & B & 752 \\
\hline 225 & 189 & F & 165897 & B & B & B & BD & B & D & $\mathrm{C}$ & $\mathrm{C}$ & $\mathrm{BC}$ & B & B & 655 \\
\hline 226 & 189 & M & 165898 & B & B & B & D & B & D & $\mathrm{C}$ & C & B & B & B & 810 \\
\hline 227 & 189 & F & 165899 & B & B & B & BD & B & D & $\mathrm{C}$ & C & B & B & B & 604 \\
\hline 228 & 189 & F & 165900 & B & B & B & B & B & D & $\mathrm{C}$ & C & B & B & B & 591 \\
\hline 229 & 189 & F & 165901 & B & B & B & BD & B & D & $\mathrm{C}$ & $\mathrm{C}$ & B & B & B & 658 \\
\hline 230) & 189 & M & 165902 & B & B & B & B & B & D & $\mathrm{C}$ & C & B & B & B & 669 \\
\hline 231 & 189 & M & 165903 & B & B & B & BD & B & D & $\mathrm{C}$ & C & B & B & B & 613 \\
\hline 232 & 189 & M & 165904 & B & B & B & BD & A & D & $\mathrm{C}$ & C & B & B & B & 670 \\
\hline 233 & 189 & $\mathrm{~F}$ & 165905 & B & B & B & $\mathrm{D}$ & B & D & $\mathrm{C}$ & $\mathrm{C}$ & B & B & B & 665 \\
\hline 234 & 189 & F & 165906 & B & B & B & BD & B & D & $\mathrm{C}$ & C & B & B & B & 721 \\
\hline 235 & 189 & M & 165907 & B & B & B & B & B & D & $\mathrm{C}$ & C & B & B & B & - \\
\hline 236 & 189 & $\mathrm{~F}$ & 165908 & B & B & B & B & B & D & $\mathrm{C}$ & $\mathrm{C}$ & B & B & B & 891 \\
\hline
\end{tabular}


APPENDIX 2-1

(Continued)

\begin{tabular}{|c|c|c|c|c|c|c|c|c|c|c|c|c|c|c|c|}
\hline & \multirow[b]{2}{*}{ Site } & \multirow[b]{2}{*}{ Sex } & \multirow[b]{2}{*}{$\mathrm{AMNH}$} & \multicolumn{12}{|c|}{ Locus } \\
\hline & & & & Aat-1 & Aat-2 & Idh-1 & Ldh-1 & Ldh-2 & Mdh-1 & Mpi & Pgi & Pgm-1 & Pgm-2 & Sod-1 & blood \\
\hline 237 & 189 & M & 165909 & B & B & B & D & B & D & $\mathrm{C}$ & $\mathrm{C}$ & B & B & B & 865 \\
\hline 238 & 189 & F & 165910 & B & B & B & BD & B & D & C & - & B & B & B & 721 \\
\hline 239 & 189 & M & 165911 & B & B & B & B & B & D & C & - & B & B & B & 620 \\
\hline 240) & 189 & M & 165912 & B & B & B & BD & B & D & C & - & B & B & B & 694 \\
\hline 241 & 189 & F & 165913 & B & B & B & D & B & $\mathrm{D}$ & C & - & B & B & B & 613 \\
\hline 242 & 189 & F & 165914 & $\mathrm{AB}$ & B & B & BD & B & D & C & - & B & B & B & 700 \\
\hline 243 & 189 & M & 165915 & B & B & B & D & B & D & C & - & B & B & B & 654 \\
\hline 244 & 189 & M & 165916 & B & B & B & D & B & D & C & - & B & B & B & 656 \\
\hline 245 & 189 & M & 165917 & $\mathrm{AB}$ & $\mathrm{BC}$ & B & BD & B & D & C & - & B & B & B & 592 \\
\hline 246 & 189 & F & 165918 & B & B & B & B & B & $\mathrm{D}$ & C & - & B & B & B & 798 \\
\hline 247 & 189 & M & 165919 & B & $\mathrm{C}$ & B & B & B & $\mathrm{D}$ & C & - & B & B & B & 651 \\
\hline 248 & 189 & $\mathrm{~F}$ & 165920 & B & B & B & B & B & $\mathrm{D}$ & C & - & B & B & B & 598 \\
\hline 249 & 189 & $\mathrm{~F}$ & 165921 & B & B & B & BD & B & D & C & - & B & B & B & 620 \\
\hline 250) & 189 & F & 165922 & B & B & B & BD & B & $\mathrm{D}$ & $\mathrm{C}$ & - & B & B & B & 641 \\
\hline 251 & 189 & M & 165923 & B & B & B & B & B & D & C & - & B & B & B & 694 \\
\hline 252 & 189 & $\mathrm{~F}$ & 165924 & B & B & B & BD & B & $\mathrm{D}$ & $\mathrm{C}$ & - & B & B & B & 782 \\
\hline 253 & 189 & M & 165925 & B & B & B & D & B & D & C & - & B & B & B & 793 \\
\hline 254 & 189 & M & 165926 & B & B & B & B & B & D & C & - & B & B & B & 686 \\
\hline 255 & 189 & $\mathrm{~F}$ & 165927 & B & B & B & D & B & D & C & - & B & B & B & 724 \\
\hline 256 & 189 & M & 165928 & B & B & B & D & B & D & C & - & B & B & B & 803 \\
\hline 257 & 189 & M & 165929 & B & B & B & D & B & D & C & - & B & B & B & 730 \\
\hline 258 & 189 & M & 165930 & B & B & B & D & B & D & C & - & B & B & B & 624 \\
\hline 259 & 189 & M & 165931 & B & B & B & B & B & D & C & - & B & B & B & 657 \\
\hline 260) & 189 & $\mathrm{~F}$ & 165932 & B & B & B & D & B & $\mathrm{D}$ & C & - & B & B & B & 698 \\
\hline 261 & 189 & M & 165933 & B & B & B & D & B & $\mathrm{D}$ & C & - & B & B & B & 660 \\
\hline 262 & 189 & $\mathrm{~F}$ & 165934 & B & B & B & BD & B & D & C & - & B & B & B & 586 \\
\hline 263 & 189 & M & 165935 & B & B & QB & D & $\mathrm{AB}$ & $\mathrm{D}$ & $\mathrm{C}$ & - & B & B & B & 790 \\
\hline 264 & 189 & $\mathrm{~F}$ & 165936 & B & B & B & D & B & D & C & - & B & B & B & 650 \\
\hline 265 & 198 & F & 158771 & B & B & B & B & B & D & C & - & B & B & B & 788 \\
\hline 266 & 198 & M & 169340 & B & B & B & B & B & D & C & - & B & B & B & FCM \\
\hline 267 & 198 & M & 169341 & B & B & B & B & B & D & C & C & B & B & B & FCM \\
\hline 268 & 198 & M & 169342 & B & B & B & B & B & CD & C & C & B & B & B & FCM \\
\hline 269 & 199 & F & 169339 & B & B & B & - & 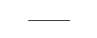 & $\mathrm{C}$ & - & - & - & B & B & - \\
\hline 270) & 204 & M & 169352 & B & B & B & B & B & D & C & C & B & B & B & FCM \\
\hline 271 & 204 & M & 169353 & B & B & B & B & B & D & C & - & B & B & B & - \\
\hline 272 & 204 & F & 169354 & B & B & B & B & B & D & - & - & B & B & B & FCM \\
\hline 273 & 204 & $\mathrm{~F}$ & 169850 & B & B & B & B & B & D & C & C & B & B & B & FCM \\
\hline 274 & 204 & F & 169851 & B & B & B & B & B & $\mathrm{D}$ & C & $\mathrm{C}$ & B & B & B & FCM \\
\hline 275 & 204 & $\mathrm{~F}$ & 169852 & B & B & B & B & B & D & C & C & B & B & B & FCM \\
\hline 276 & 204 & $\mathrm{~F}$ & 169853 & B & B & B & B & B & D & C & C & B & B & B & FCM \\
\hline 277 & 204 & M & 169854 & B & B & B & B & B & D & C & $\mathrm{AC}$ & B & B & B & FCM \\
\hline 278 & 204 & M & 169857 & B & B & B & B & B & D & C & A & B & B & B & FCM \\
\hline 279 & 204 & M & 169858 & B & B & B & B & B & D & C & C & B & B & B & FCM \\
\hline 280) & 204 & M & 169859 & B & B & B & B & B & D & C & C & B & B & B & FCM \\
\hline 281 & 204 & M & 169860 & B & B & B & B & A & D & C & C & B & B & B & FCM \\
\hline 282 & 204 & M & 169861 & B & B & B & B & A & $\mathrm{D}$ & C & A & B & B & B & FCM \\
\hline 283 & 204 & M & 169862 & B & B & B & B & A & $\mathrm{D}$ & C & C & B & B & B & FCM \\
\hline 284 & 204 & M & 169863 & B & B & B & B & B & D & C & C & B & B & B & FCM \\
\hline 285 & 204 & M & 169864 & B & B & B & B & A & $\mathrm{D}$ & C & C & B & B & B & FCM \\
\hline 286 & 204 & M & 169865 & B & B & B & B & B & D & C & $\mathrm{AC}$ & B & B & B & FCM \\
\hline 287 & 204 & M & 169866 & B & B & B & B & B & $\mathrm{D}$ & $\mathrm{C}$ & A & B & B & B & FCM \\
\hline 288 & 204 & M & 169867 & B & B & B & B & B & D & C & A & B & B & B & FCM \\
\hline
\end{tabular}


APPENDIX 2-1

(Continued)

Locus

Site Sex AMNH Aat-1 Aat-2 Idh-1 Ldh-1 Ldh-2 Mdh-1 Mpi Pgi Pgm-1 Pgm-2 Sod-1 blood

\begin{tabular}{llllllllllllllll}
\hline 289 & 204 & $\mathrm{~F}$ & 169868 & $\mathrm{~B}$ & $\mathrm{~B}$ & $\mathrm{~B}$ & $\mathrm{~B}$ & $\mathrm{~A}$ & $\mathrm{D}$ & $\mathrm{C}$ & $\mathrm{C}$ & $\mathrm{B}$ & $\mathrm{B}$ & $\mathrm{B}$ & $\mathrm{FCM}$ \\
$290)$ & 206 & $\mathrm{M}$ & 169367 & $\mathrm{~B}$ & $\mathrm{~B}$ & $\mathrm{~B}$ & $\mathrm{BC}$ & $\mathrm{B}$ & $\mathrm{D}$ & $\mathrm{C}$ & $\mathrm{C}$ & $\mathrm{B}$ & $\mathrm{B}$ & $\mathrm{B}$ & $\mathrm{FCM}$ \\
291 & 206 & $\mathrm{~F}$ & 169869 & $\mathrm{~B}$ & $\mathrm{~B}$ & $\mathrm{~B}$ & $\mathrm{~B}$ & $\mathrm{~B}$ & $\mathrm{D}$ & $\mathrm{C}$ & $\mathrm{C}$ & $\mathrm{B}$ & $\mathrm{B}$ & $\mathrm{B}$ & $\mathrm{FCM}$ \\
292 & 210 & $\mathrm{~F}$ & 158772 & $\mathrm{~B}$ & $\mathrm{~B}$ & $\mathrm{~B}$ & $\mathrm{~B}$ & $\mathrm{~B}$ & $\mathrm{D}$ & $\mathrm{C}$ & $\mathrm{C}$ & $\mathrm{B}$ & $\mathrm{B}$ & $\mathrm{B}$ & 605 \\
293 & 210 & $\mathrm{M}$ & 158773 & $\mathrm{~B}$ & $\mathrm{~B}$ & $\mathrm{~B}$ & $\mathrm{~B}$ & $\mathrm{~B}$ & $\mathrm{D}$ & $\mathrm{C}$ & $\mathrm{C}$ & $\mathrm{B}$ & $\mathrm{B}$ & $\mathrm{B}$ & 671 \\
294 & 210 & $\mathrm{M}$ & 158774 & $\mathrm{~B}$ & $\mathrm{~B}$ & $\mathrm{~B}$ & $\mathrm{~B}$ & $\mathrm{~B}$ & $\mathrm{D}$ & - & $\mathrm{C}$ & $\mathrm{B}$ & $\mathrm{B}$ & $\mathrm{B}$ & 729 \\
295 & 210 & $\mathrm{~F}$ & 158775 & $\mathrm{~B}$ & $\mathrm{~B}$ & $\mathrm{~B}$ & - & $\mathrm{B}$ & $\mathrm{D}$ & - & - & $\mathrm{B}$ & $\mathrm{B}$ & $\mathrm{B}$ & 728 \\
296 & 210 & $\mathrm{M}$ & 158776 & $\mathrm{~B}$ & $\mathrm{~B}$ & $\mathrm{~B}$ & $\mathrm{~B}$ & $\mathrm{~B}$ & $\mathrm{D}$ & - & - & $\mathrm{B}$ & $\mathrm{B}$ & $\mathrm{B}$ & 728 \\
297 & 211 & $\mathrm{~F}$ & 158777 & $\mathrm{~B}$ & $\mathrm{~B}$ & $\mathrm{~B}$ & - & $\mathrm{B}$ & $\mathrm{D}$ & - & - & $\mathrm{B}$ & $\mathrm{B}$ & $\mathrm{B}$ & 650 \\
298 & 211 & $\mathrm{M}$ & 158778 & $\mathrm{~B}$ & $\mathrm{~B}$ & $\mathrm{~B}$ & $\mathrm{~B}$ & $\mathrm{~B}$ & $\mathrm{D}$ & - & - & $\mathrm{B}$ & $\mathrm{B}$ & $\mathrm{B}$ & 622 \\
299 & 211 & $\mathrm{M}$ & 159991 & $\mathrm{~B}$ & $\mathrm{~B}$ & $\mathrm{~B}$ & $\mathrm{~B}$ & $\mathrm{~B}$ & $\mathrm{D}$ & $\mathrm{C}$ & - & $\mathrm{B}$ & $\mathrm{B}$ & $\mathrm{B}$ & 683 \\
$300)$ & 211 & $\mathrm{M}$ & 159992 & $\mathrm{~B}$ & $\mathrm{~B}$ & $\mathrm{~B}$ & $\mathrm{~B}$ & $\mathrm{~B}$ & $\mathrm{D}$ & $\mathrm{C}$ & - & $\mathrm{B}$ & $\mathrm{B}$ & $\mathrm{B}$ & 713 \\
301 & 211 & $\mathrm{M}$ & 159993 & $\mathrm{~B}$ & $\mathrm{~B}$ & $\mathrm{~B}$ & $\mathrm{~B}$ & $\mathrm{~B}$ & $\mathrm{D}$ & $\mathrm{C}$ & - & $\mathrm{B}$ & $\mathrm{B}$ & $\mathrm{B}$ & 721 \\
302 & 211 & $\mathrm{M}$ & 159994 & $\mathrm{~B}$ & $\mathrm{~B}$ & $\mathrm{~B}$ & $\mathrm{~B}$ & $\mathrm{~B}$ & $\mathrm{D}$ & $\mathrm{C}$ & - & $\mathrm{B}$ & $\mathrm{B}$ & $\mathrm{B}$ & 661 \\
303 & 211 & $\mathrm{M}$ & 159995 & $\mathrm{~B}$ & $\mathrm{~B}$ & $\mathrm{~B}$ & $\mathrm{~B}$ & $\mathrm{~B}$ & $\mathrm{D}$ & $\mathrm{C}$ & - & $\mathrm{B}$ & $\mathrm{B}$ & $\mathrm{B}$ & 708 \\
304 & 211 & $\mathrm{M}$ & 159996 & $\mathrm{~B}$ & $\mathrm{~B}$ & $\mathrm{~B}$ & $\mathrm{~B}$ & $\mathrm{~B}$ & $\mathrm{D}$ & $\mathrm{C}$ & - & $\mathrm{B}$ & $\mathrm{B}$ & $\mathrm{B}$ & 724 \\
305 & 211 & $\mathrm{M}$ & 159997 & $\mathrm{~B}$ & $\mathrm{~B}$ & $\mathrm{~B}$ & $\mathrm{~B}$ & $\mathrm{~B}$ & $\mathrm{D}$ & $\mathrm{C}$ & - & $\mathrm{B}$ & $\mathrm{B}$ & $\mathrm{B}$ & 723 \\
306 & 211 & $\mathrm{M}$ & 159998 & $\mathrm{~B}$ & $\mathrm{~B}$ & $\mathrm{~B}$ & $\mathrm{~B}$ & $\mathrm{~B}$ & $\mathrm{D}$ & $\mathrm{C}$ & - & $\mathrm{B}$ & $\mathrm{B}$ & $\mathrm{B}$ & 782 \\
307 & 214 & $\mathrm{~F}$ & 158769 & $\mathrm{~B}$ & $\mathrm{~B}$ & $\mathrm{~B}$ & $\mathrm{~B}$ & $\mathrm{~B}$ & $\mathrm{D}$ & $\mathrm{C}$ & - & $\mathrm{B}$ & $\mathrm{B}$ & $\mathrm{B}$ & 689 \\
308 & 215 & $\mathrm{M}$ & 158770 & $\mathrm{~B}$ & $\mathrm{~B}$ & $\mathrm{~B}$ & $\mathrm{~B}$ & $\mathrm{~B}$ & $\mathrm{D}$ & $\mathrm{C}$ & $\mathrm{C}$ & $\mathrm{B}$ & $\mathrm{B}$ & $\mathrm{B}$ & 750 \\
\hline
\end{tabular}

Appendix 2-2: Genotypes of diploid (2n) Ambystoma jeffersonianum specimens ordered by site number.

\begin{tabular}{|c|c|c|c|c|c|c|c|c|c|c|c|c|c|c|c|}
\hline & \multirow[b]{2}{*}{ Site } & \multirow[b]{2}{*}{ Sex } & \multirow[b]{2}{*}{ AMNH } & \multicolumn{12}{|c|}{ Locus } \\
\hline & & & & Aat-1 & Aat-2 & Idh-1 & Ldh-1 & Ldh-2 & Mdh-1 & Mpi & Pgi & Pgm-1 & Pgm-2 & Sod-1 & blood \\
\hline 1 & 2 & M & 160166 & D & A & A & $\mathrm{C}$ & B & B & $\mathrm{BC}$ & $\mathrm{AB}$ & B & B & D & 627 \\
\hline 2 & 2 & M & 160167 & D & A & A & $\mathrm{C}$ & B & B & B & A & $\mathrm{C}$ & B & D & 798 \\
\hline 3 & 2 & M & 160168 & D & A & A & $\mathrm{C}$ & B & B & $\mathrm{BC}$ & - & $\mathrm{C}$ & B & D & 776 \\
\hline 4 & 2 & $\mathrm{~F}$ & 160169 & D & A & A & $\mathrm{C}$ & B & B & $\mathrm{BC}$ & - & $\mathrm{BC}$ & B & D & 814 \\
\hline 5 & 2 & M & 160170 & D & A & A & C & B & BD & $\mathrm{BC}$ & - & B & B & D & 802 \\
\hline 6 & 2 & M & 160171 & D & A & A & $\mathrm{C}$ & B & $\mathrm{D}$ & - & $\mathrm{AB}$ & $\mathrm{C}$ & B & D & 628 \\
\hline 7 & 2 & M & 160172 & D & A & A & $\mathrm{C}$ & B & D & - & $\mathrm{AB}$ & $\mathrm{BC}$ & B & $\mathrm{D}$ & 731 \\
\hline 8 & 2 & $\mathrm{M}$ & 160173 & D & A & A & C & B & B & - & A & $\mathrm{BC}$ & B & $\mathrm{D}$ & 783 \\
\hline 9 & 2 & M & 160174 & D & A & A & C & B & B & $\mathrm{BC}$ & - & $\mathrm{BC}$ & B & D & 719 \\
\hline 10) & 2 & M & 160175 & D & A & A & $\mathrm{C}$ & B & $\mathrm{BD}$ & $\mathrm{BC}$ & - & B & B & $\mathrm{D}$ & 693 \\
\hline 11 & 2 & $\mathrm{~F}$ & 160176 & D & A & A & $\mathrm{C}$ & B & $\mathrm{BD}$ & - & A & $\mathrm{BC}$ & B & $\mathrm{D}$ & 558 \\
\hline 12 & 2 & $\mathrm{M}$ & 160177 & D & A & A & C & BQ & BD & - & $\mathrm{AB}$ & C & B & D & 747 \\
\hline 13 & 2 & M & 160178 & D & A & A & $\mathrm{C}$ & B & BD & - & $\mathrm{AB}$ & C & B & D & 840 \\
\hline 14 & 2 & M & 160179 & D & A & A & $\mathrm{C}$ & BQ & B & - & $\mathrm{AB}$ & $\mathrm{BC}$ & B & D & 790 \\
\hline 15 & 2 & $\mathrm{M}$ & 160180 & D & A & A & $\mathrm{C}$ & B & BD & - & B & $\mathrm{BC}$ & B & D & 776 \\
\hline 16 & 2 & M & 160181 & D & A & A & $\mathrm{C}$ & B & BD & - & B & $\mathrm{BC}$ & B & D & 810 \\
\hline 17 & 2 & M & 160182 & D & A & A & $\mathrm{C}$ & B & $\mathrm{BD}$ & - & $\mathrm{AB}$ & B & B & $\mathrm{D}$ & 789 \\
\hline 18 & 2 & $\mathrm{~F}$ & 160183 & D & A & A & C & B & B & - & $\mathrm{AB}$ & B & B & D & 742 \\
\hline 19 & 2 & M & 160184 & D & A & A & $\mathrm{C}$ & B & B & - & A & B & B & D & 717 \\
\hline 20) & 2 & M & 160185 & D & A & A & C & B & B & - & A & $\mathrm{BC}$ & B & D & 801 \\
\hline 21 & 2 & $\mathrm{~F}$ & 160186 & D & A & A & C & B & BD & - & $\mathrm{AB}$ & $\mathrm{BC}$ & B & D & 758 \\
\hline 22 & 2 & M & 160187 & D & A & A & $\mathrm{C}$ & B & D & 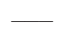 & $\mathrm{AB}$ & $\mathrm{P}$ & B & D & 774 \\
\hline
\end{tabular}


APPENDIX 2-2

(Continued)

\begin{tabular}{|c|c|c|c|c|c|c|c|c|c|c|c|c|c|c|c|}
\hline & \multirow[b]{2}{*}{ Site } & \multirow[b]{2}{*}{ Sex } & \multirow[b]{2}{*}{ AMNH } & \multicolumn{12}{|c|}{ Locus } \\
\hline & & & & Aat-1 & Aat-2 & Idh-1 & Ldh-1 & Ldh-2 & Mdh-1 & Mpi & Pgi & Pgm-1 & Pgm-2 & Sod-1 & blood \\
\hline 23 & 2 & M & 160188 & D & A & A & C & B & BD & - & A & $\mathrm{BC}$ & B & D & 777 \\
\hline 24 & 2 & $\mathrm{~F}$ & 160189 & D & A & A & C & B & B & - & $\mathrm{AB}$ & B & B & D & 759 \\
\hline 25 & 2 & M & 160190 & D & A & A & C & B & B & $\mathrm{BC}$ & - & $\mathrm{BC}$ & B & D & 807 \\
\hline 26 & 2 & $\mathrm{~F}$ & 160191 & D & A & A & C & B & B & $\mathrm{BC}$ & - & C & B & D & 723 \\
\hline 27 & 2 & M & 160192 & D & A & A & C & B & BD & B & - & B & B & D & 780 \\
\hline 28 & 2 & $\mathrm{~F}$ & 160193 & D & A & A & C & B & B & B & - & C & B & D & 772 \\
\hline 29 & 2 & $\mathrm{~F}$ & 160194 & D & A & A & C & B & BD & B & - & C & B & D & 837 \\
\hline 30) & 2 & M & 160195 & D & A & A & C & B & $\mathrm{BD}$ & B & - & $\mathrm{BC}$ & B & D & 768 \\
\hline 31 & 2 & $\mathrm{~F}$ & 160196 & D & A & A & C & B & B & $\mathrm{BC}$ & - & $\mathrm{BC}$ & B & D & 650 \\
\hline 32 & 2 & M & 160197 & D & A & A & C & B & B & $\mathrm{BC}$ & - & $\mathrm{BC}$ & B & D & 744 \\
\hline 33 & 2 & M & 160198 & D & A & A & $\mathrm{C}$ & B & BD & B & - & $\mathrm{BC}$ & B & D & 864 \\
\hline 34 & 2 & M & 160199 & D & A & A & C & B & BD & B & - & $\mathrm{BC}$ & B & D & 816 \\
\hline 35 & 2 & $\mathrm{~F}$ & 160200 & D & A & A & C & B & B & $\mathrm{BC}$ & - & C & B & D & - \\
\hline 36 & 114 & $\mathrm{~F}$ & 153123 & D & A & A & C & B & B & B & - & $\mathrm{BC}$ & B & D & 660 \\
\hline 37 & 114 & $\mathrm{~F}$ & 153124 & D & A & A & C & B & B & - & - & C & B & D & 794 \\
\hline 38 & 114 & M & 153125 & D & A & A & C & B & B & - & - & C & B & D & 602 \\
\hline 39 & 114 & M & 153126 & D & A & A & C & B & B & - & - & $\mathrm{BC}$ & B & D & 704 \\
\hline 40) & 114 & M & 153127 & D & A & A & C & B & B & 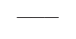 & - & $\mathrm{BC}$ & B & D & 683 \\
\hline 41 & 114 & M & 153128 & D & A & A & C & B & B & B & - & B & B & D & 848 \\
\hline 42 & 114 & $\mathrm{~F}$ & 153129 & D & A & A & C & B & B & B & - & $\mathrm{BC}$ & B & D & 805 \\
\hline 43 & 114 & M & 153130 & D & A & A & C & B & B & B & - & $\mathrm{BC}$ & B & D & 785 \\
\hline 44 & 114 & M & 153131 & D & A & A & C & B & B & B & - & $\mathrm{BC}$ & B & D & 701 \\
\hline 45 & 114 & $\mathrm{~F}$ & 153132 & D & A & A & C & B & B & 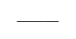 & - & $\mathrm{BC}$ & B & D & 720 \\
\hline 46 & 114 & $\mathrm{~F}$ & 153133 & D & A & A & C & B & B & - & - & B & B & D & 672 \\
\hline 47 & 114 & $\mathrm{M}$ & 153134 & D & A & A & C & B & B & - & - & B & B & D & 656 \\
\hline 48 & 114 & $\mathrm{~F}$ & 153135 & D & A & A & C & B & B & - & - & B & B & D & 731 \\
\hline 49 & 114 & $\mathrm{~F}$ & 153136 & D & A & A & C & B & B & B & - & C & B & D & 753 \\
\hline 50) & 114 & $\mathrm{M}$ & 153137 & D & A & A & C & B & B & B & - & $\mathrm{BC}$ & B & D & 668 \\
\hline 51 & 114 & $\mathrm{~F}$ & 153138 & D & A & A & C & B & B & B & - & C & B & D & 718 \\
\hline 52 & 114 & $\mathrm{M}$ & 153139 & D & A & A & C & B & B & B & - & $\mathrm{BC}$ & B & D & 785 \\
\hline 53 & 114 & $\mathrm{~F}$ & 153140 & D & A & A & C & B & B & - & - & $\mathrm{BC}$ & B & D & 682 \\
\hline 54 & 114 & $\mathrm{~F}$ & 153141 & D & A & A & C & B & B & - & - & B & B & D & 665 \\
\hline 55 & 114 & $\mathrm{~F}$ & 153142 & D & A & A & C & B & B & - & - & C & B & D & 680 \\
\hline 56 & 114 & $\mathrm{~F}$ & 153143 & D & A & A & C & B & B & - & - & C & B & D & 749 \\
\hline 57 & 114 & M & 153144 & D & A & A & C & B & B & 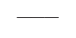 & - & C & B & D & 708 \\
\hline 58 & 114 & $\mathrm{~F}$ & 153145 & D & A & A & C & B & B & B & A & C & B & D & 695 \\
\hline 59 & 114 & $\mathrm{M}$ & 153146 & D & A & A & C & B & B & B & - & B & B & D & 724 \\
\hline 60) & 114 & $\mathrm{~F}$ & 153147 & D & A & A & C & B & B & 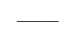 & - & B & B & D & 670 \\
\hline 61 & 114 & $\mathrm{~F}$ & 153148 & D & A & A & C & B & B & - & - & $\mathrm{BC}$ & B & D & 654 \\
\hline 62 & 114 & M & 153149 & D & A & A & $\mathrm{C}$ & B & B & - & A & $\mathrm{BC}$ & B & D & 591 \\
\hline 63 & 114 & $\mathrm{M}$ & 153150 & D & A & A & C & B & B & - & A & $\mathrm{BC}$ & B & D & 716 \\
\hline 64 & 114 & $\mathrm{~F}$ & 153151 & D & A & A & C & B & B & 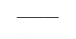 & A & $\mathrm{BC}$ & B & D & 668 \\
\hline 65 & 114 & M & 153152 & D & A & A & C & B & B & B & - & $\mathrm{BC}$ & B & D & 788 \\
\hline 66 & 114 & $\mathrm{~F}$ & 153153 & D & A & A & C & B & B & B & - & C & B & D & 732 \\
\hline 67 & 114 & $\mathrm{~F}$ & 153154 & D & A & A & C & B & B & - & - & $\mathrm{BC}$ & B & D & 767 \\
\hline 68 & 114 & $?$ & 153155 & D & A & A & C & B & B & - & - & C & B & D & 669 \\
\hline 69 & 114 & $\mathrm{~F}$ & 153156 & D & A & A & C & B & B & B & - & $\mathrm{BC}$ & B & D & 766 \\
\hline 70) & 114 & $\mathrm{M}$ & 153157 & D & A & A & C & B & B & - & - & $\mathrm{BC}$ & B & D & 589 \\
\hline 71 & 114 & $\mathrm{~F}$ & 153158 & D & A & A & C & B & B & - & - & C & B & D & 662 \\
\hline 72 & 114 & M & 153159 & D & A & A & C & B & B & - & B & $\mathrm{BC}$ & B & D & 641 \\
\hline 73 & 114 & $\mathrm{~F}$ & 160239 & D & A & A & C & B & B & B & B & C & B & D & 723 \\
\hline 74 & 114 & $\mathrm{M}$ & 160240 & D & A & A & C & B & B & B & - & C & B & D & 667 \\
\hline
\end{tabular}


Locus

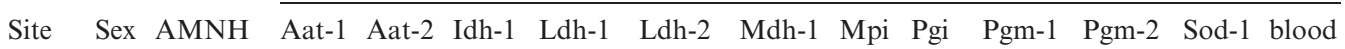

\begin{tabular}{|c|c|c|c|c|c|c|c|c|c|c|c|c|c|c|c|}
\hline 75 & 114 & M & 160241 & $\mathrm{D}$ & A & A & $\mathrm{C}$ & B & B & B & - & $\mathrm{BC}$ & B & D & 803 \\
\hline 76 & 114 & M & 160242 & $\mathrm{D}$ & A & A & $\mathrm{C}$ & B & B & B & - & $\mathrm{BC}$ & B & $\mathrm{D}$ & 765 \\
\hline 77 & 114 & $\mathrm{~F}$ & 160243 & $\mathrm{D}$ & A & A & $\mathrm{C}$ & B & B & B & - & $\mathrm{C}$ & B & D & 700 \\
\hline 78 & 114 & $\mathrm{~F}$ & 160244 & D & A & A & $\mathrm{C}$ & B & B & B & - & B & B & D & 717 \\
\hline 79 & 114 & $\mathrm{~F}$ & 160245 & D & A & A & $\mathrm{C}$ & B & B & B & - & $\mathrm{BC}$ & B & $\mathrm{D}$ & 805 \\
\hline 80) & 115 & $\mathrm{~F}$ & 153122 & D & A & A & $\mathrm{C}$ & B & B & - & $\mathrm{AB}$ & $\mathrm{C}$ & B & D & 675 \\
\hline 81 & 116 & $\mathrm{~F}$ & 169460 & D & A & A & $\mathrm{C}$ & B & B & B & - & $\mathrm{BC}$ & B & D & FCM \\
\hline 82 & 118 & M & 160223 & D & A & A & $\mathrm{C}$ & B & B & - & B & C & B & D & 743 \\
\hline 83 & 118 & M & 160224 & D & A & A & $\mathrm{C}$ & B & BD & - & $\mathrm{AB}$ & C & B & D & 784 \\
\hline 84 & 118 & M & 160225 & D & A & A & $\mathrm{C}$ & B & BD & - & QA & $\mathrm{C}$ & B & $\mathrm{D}$ & 699 \\
\hline 85 & 118 & M & 160226 & D & A & A & $\mathrm{C}$ & B & B & - & B & C & B & D & 740 \\
\hline 86 & 118 & M & 160227 & D & A & QA & C & B & BD & - & $\mathrm{AB}$ & C & B & D & 824 \\
\hline 87 & 118 & $\mathrm{~F}$ & 160228 & D & - & A & $\mathrm{C}$ & B & B & - & $\mathrm{AB}$ & B & B & D & 652 \\
\hline 88 & 118 & M & 160229 & D & A & A & $\mathrm{C}$ & B & BD & - & - & $\mathrm{C}$ & B & D & 737 \\
\hline 89 & 118 & $\mathrm{~F}$ & 160230 & D & A & A & $\mathrm{C}$ & B & BD & - & - & $\mathrm{C}$ & B & D & 676 \\
\hline 90) & 118 & M & 160231 & D & A & A & C & B & D & - & $\mathrm{AB}$ & C & B & D & 652 \\
\hline 91 & 118 & $\mathrm{~F}$ & 160232 & D & A & A & C & B & $\mathrm{BD}$ & - & $\mathrm{AB}$ & C & B & D & 712 \\
\hline 92 & 118 & $\mathrm{~F}$ & 160233 & D & A & A & $\mathrm{C}$ & B & BD & - & $\mathrm{AB}$ & $\mathrm{C}$ & B & D & 708 \\
\hline 93 & 118 & M & 160234 & D & A & A & $\mathrm{C}$ & B & $\mathrm{D}$ & B & $\mathrm{AB}$ & $\mathrm{C}$ & B & D & 709 \\
\hline 94 & 118 & M & 160235 & D & A & A & $\mathrm{C}$ & B & $\mathrm{BD}$ & - & $\mathrm{AB}$ & $\mathrm{BC}$ & B & D & 896 \\
\hline 95 & 118 & $\mathrm{~F}$ & 160236 & D & A & A & $\mathrm{C}$ & B & D & - & $\mathrm{AB}$ & C & B & D & 712 \\
\hline 96 & 118 & M & 160237 & D & A & A & $\mathrm{C}$ & B & B & - & B & C & B & D & 704 \\
\hline 97 & 118 & $\mathrm{~F}$ & 160238 & D & A & A & $\mathrm{C}$ & B & B & - & $\mathrm{AB}$ & $\mathrm{C}$ & B & D & 728 \\
\hline 98 & 119 & $\mathrm{~F}$ & 160222 & D & A & A & $\mathrm{C}$ & B & BD & $\mathrm{BC}$ & - & C & B & D & 945 \\
\hline 99 & 120 & M & 163376 & D & A & A & $\mathrm{C}$ & B & BD & $\mathrm{BC}$ & $\mathrm{AB}$ & $\mathrm{C}$ & B & D & 780 \\
\hline 100) & 120 & $\mathrm{~F}$ & 163377 & D & A & A & C & B & D & B & A & C & B & D & 724 \\
\hline 101 & 120 & $\mathrm{~F}$ & 163378 & D & A & A & $\mathrm{C}$ & B & D & B & B & $\mathrm{BC}$ & B & D & 686 \\
\hline 102 & 120 & M & 163379 & D & A & A & $\mathrm{C}$ & B & BD & $\mathrm{BC}$ & $\mathrm{AB}$ & $\mathrm{BC}$ & B & D & 687 \\
\hline 103 & 120 & $\mathrm{~F}$ & 163380 & D & A & A & $\mathrm{C}$ & BQ & BD & B & $\mathrm{AB}$ & B & B & $\mathrm{D}$ & 725 \\
\hline 104 & 120 & $\mathrm{~F}$ & 163381 & D & A & A & C & B & BD & - & - & $\mathrm{BC}$ & B & D & 678 \\
\hline 105 & 120 & $\mathrm{~F}$ & 163382 & D & A & A & $\mathrm{C}$ & B & BD & B & B & $\mathrm{BC}$ & B & D & 852 \\
\hline 106 & 120 & $\mathrm{~F}$ & 163383 & D & A & A & $\mathrm{C}$ & B & B & B & $\mathrm{AB}$ & $\mathrm{C}$ & B & D & 798 \\
\hline 107 & 120 & $\mathrm{~F}$ & 163384 & D & A & A & C & B & $\mathrm{BD}$ & $\mathrm{BC}$ & - & C & B & D & 699 \\
\hline 108 & 120 & $\mathrm{~F}$ & 163385 & D & A & A & $\mathrm{C}$ & B & B & B & $\mathrm{AB}$ & C & B & D & 769 \\
\hline 109 & 120 & M & 163386 & D & A & A & $\mathrm{C}$ & B & $\mathrm{BD}$ & B & B & B & B & D & 869 \\
\hline 110) & 120 & $\mathrm{~F}$ & 163387 & D & A & A & $\mathrm{C}$ & B & B & $\mathrm{BC}$ & - & C & B & D & 774 \\
\hline 111 & 120 & M & 163388 & D & A & A & $\mathrm{C}$ & B & B & - & A & $\mathrm{BC}$ & B & D & 781 \\
\hline 112 & 120 & M & 163389 & D & A & A & $\mathrm{C}$ & B & BD & $\mathrm{BC}$ & $\mathrm{AB}$ & C & B & D & 734 \\
\hline 113 & 121 & M & 160202 & D & A & A & $\mathrm{C}$ & B & B & B & $\mathrm{AB}$ & $\mathrm{BC}$ & B & D & 674 \\
\hline 114 & 121 & M & 160203 & D & A & A & $\mathrm{C}$ & B & B & B & $\mathrm{AB}$ & $\mathrm{C}$ & B & D & 626 \\
\hline 115 & 121 & M & 160204 & $\mathrm{D}$ & A & A & $\mathrm{C}$ & B & B & B & - & C & B & $\mathrm{D}$ & 662 \\
\hline 116 & 121 & M & 160205 & $\mathrm{D}$ & A & A & $\mathrm{C}$ & B & B & B & $\mathrm{AB}$ & $\mathrm{BC}$ & B & $\mathrm{D}$ & 698 \\
\hline 117 & 121 & M & 160206 & D & A & A & C & B & B & B & A & $\mathrm{BC}$ & B & D & 746 \\
\hline 118 & 121 & M & 160207 & D & A & A & C & B & B & B & $\mathrm{AB}$ & $\mathrm{BC}$ & B & D & 753 \\
\hline 119 & 121 & M & 160208 & D & A & A & $\mathrm{C}$ & B & B & B & $\mathrm{AB}$ & $\mathrm{C}$ & B & $\mathrm{D}$ & 700 \\
\hline 120) & 121 & M & 160209 & D & A & A & C & B & B & B & A & $\mathrm{C}$ & B & D & 554 \\
\hline 121 & 121 & $\mathrm{~F}$ & 160210 & D & A & A & $\mathrm{C}$ & B & B & - & $\mathrm{AB}$ & C & B & D & - \\
\hline 122 & 121 & $\mathrm{~F}$ & 160211 & D & A & A & $\mathrm{C}$ & B & B & B & - & $\mathrm{BC}$ & B & $\mathrm{D}$ & - \\
\hline 123 & 121 & M & 160212 & D & A & A & $\mathrm{C}$ & B & B & B & - & C & B & D & - \\
\hline 124 & 121 & $\mathrm{~F}$ & 160213 & D & A & A & $\mathrm{C}$ & B & B & B & - & $\mathrm{AC}$ & B & $\mathrm{D}$ & 725 \\
\hline 125 & 121 & M & 160214 & D & A & A & $\mathrm{C}$ & B & B & B & - & $\mathrm{BC}$ & B & $\mathrm{D}$ & 725 \\
\hline 126 & 121 & M & 160215 & $\mathrm{D}$ & A & A & $\mathrm{C}$ & B & B & B & - & $\mathrm{C}$ & B & D & 853 \\
\hline
\end{tabular}


APPENDIX 2-2

(Continued)

Locus

Site Sex AMNH Aat-1 Aat-2 Idh-1 Ldh-1 Ldh-2 Mdh-1 Mpi Pgi Pgm-1 Pgm-2 Sod-1 blood

\begin{tabular}{|c|c|c|c|c|c|c|c|c|c|c|c|c|c|c|c|}
\hline 127 & 121 & $\mathrm{~F}$ & 160216 & $\mathrm{D}$ & A & A & $\mathrm{C}$ & $\mathrm{B}$ & B & B & - & $\mathrm{C}$ & B & $\mathrm{D}$ & 617 \\
\hline 128 & 121 & $\mathrm{~F}$ & 160217 & $\mathrm{D}$ & A & A & $\mathrm{C}$ & B & B & B & - & $\mathrm{BC}$ & B & $\mathrm{D}$ & 773 \\
\hline 129 & 121 & M & 160218 & $\mathrm{D}$ & A & A & $\mathrm{C}$ & B & B & B & - & $\mathrm{C}$ & B & $\mathrm{D}$ & 835 \\
\hline 130) & 121 & M & 160219 & $\mathrm{D}$ & A & A & $\mathrm{C}$ & $\mathrm{B}$ & B & B & - & $\mathrm{BC}$ & B & $\mathrm{D}$ & 734 \\
\hline 131 & 121 & $\mathrm{~F}$ & 160220 & $\mathrm{D}$ & A & A & $\mathrm{C}$ & $\mathrm{B}$ & B & B & - & $\mathrm{BC}$ & B & $\mathrm{D}$ & 726 \\
\hline 132 & 121 & $\mathrm{~F}$ & 160221 & $\mathrm{D}$ & A & A & $\mathrm{C}$ & B & B & B & - & B & B & $\mathrm{D}$ & 734 \\
\hline 133 & 123 & M & 160201 & $\mathrm{D}$ & $\mathrm{A}$ & A & $\mathrm{C}$ & $\mathrm{B}$ & B & 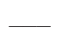 & A & $\mathrm{C}$ & B & $\mathrm{D}$ & 747 \\
\hline 134 & 124 & $\mathrm{~F}$ & 169823 & $\mathrm{D}$ & A & A & $\mathrm{C}$ & B & B & B & A & $\mathrm{C}$ & B & $\mathrm{D}$ & FCM \\
\hline 135 & 125 & $\mathrm{~F}$ & 169824 & $\mathrm{D}$ & A & A & $\mathrm{C}$ & B & B & B & $\mathrm{AB}$ & $\mathrm{C}$ & B & $\mathrm{D}$ & FCM \\
\hline 136 & 125 & $\mathrm{~F}$ & 169825 & $\mathrm{D}$ & A & A & $\mathrm{C}$ & B & B & B & - & $\mathrm{C}$ & B & $\mathrm{D}$ & FCM \\
\hline 137 & 126 & $\mathrm{~F}$ & 169797 & $\mathrm{D}$ & A & A & $\mathrm{C}$ & $\mathrm{B}$ & B & B & - & $\mathrm{C}$ & $\mathrm{B}$ & $\mathrm{D}$ & FCM \\
\hline 138 & 126 & $\mathrm{~F}$ & 169798 & $\mathrm{D}$ & A & A & $\mathrm{C}$ & B & B & B & A & B & B & $\mathrm{D}$ & FCM \\
\hline 139 & 126 & M & 169799 & $\mathrm{D}$ & A & A & $\mathrm{C}$ & B & B & B & A & $\mathrm{BC}$ & B & $\mathrm{D}$ & FCM \\
\hline 140) & 126 & M & 169800 & $\mathrm{D}$ & A & A & $\mathrm{C}$ & B & B & A & - & $\mathrm{BC}$ & $\mathrm{B}$ & $\mathrm{D}$ & FCM \\
\hline 141 & 126 & M & 169801 & $\mathrm{D}$ & A & A & $\mathrm{C}$ & B & B & B & A & $\mathrm{BC}$ & $\mathrm{B}$ & $\mathrm{D}$ & $\mathrm{FCM}$ \\
\hline 142 & 130 & $\mathrm{~F}$ & 163372 & $\mathrm{D}$ & $\mathrm{A}$ & A & $\mathrm{C}$ & B & B & B & $\mathrm{AB}$ & $\mathrm{B}$ & $\mathrm{B}$ & $\mathrm{D}$ & 860 \\
\hline 143 & 130 & $\mathrm{~F}$ & 165826 & $\mathrm{D}$ & A & A & $\mathrm{C}$ & B & B & B & $\mathrm{C}$ & $\mathrm{C}$ & B & $\mathrm{D}$ & 813 \\
\hline 144 & 130 & $\mathrm{~F}$ & 165827 & $\mathrm{D}$ & A & A & $\mathrm{C}$ & $\mathrm{B}$ & $\mathrm{BD}$ & B & - & $\mathrm{C}$ & B & $\mathrm{D}$ & 853 \\
\hline 145 & 130 & $\mathrm{~F}$ & 165828 & $\mathrm{D}$ & A & A & $\mathrm{C}$ & B & B & B & $\mathrm{C}$ & $\mathrm{C}$ & B & $\mathrm{D}$ & 754 \\
\hline 146 & 130 & $\mathrm{~F}$ & 165829 & $\mathrm{D}$ & A & A & $\mathrm{C}$ & B & B & B & $\mathrm{AC}$ & $\mathrm{C}$ & B & $\mathrm{D}$ & 748 \\
\hline 147 & 130 & M & 165830 & $\mathrm{D}$ & A & A & $\mathrm{C}$ & B & B & B & $\mathrm{AB}$ & $\mathrm{C}$ & B & $\mathrm{D}$ & 796 \\
\hline 148 & 131 & M & 165831 & $\mathrm{D}$ & A & A & $\mathrm{C}$ & B & B & B & - & $\mathrm{C}$ & B & $\mathrm{D}$ & 723 \\
\hline 149 & 135 & M & 163467 & $\mathrm{D}$ & A & A & $\mathrm{AC}$ & $\mathrm{B}$ & B & B & $\mathrm{AB}$ & $\mathrm{C}$ & B & $\mathrm{D}$ & - \\
\hline 150) & 137 & $?$ & JPB 31109 & $\mathrm{D}$ & A & A & $\mathrm{C}$ & B & B & B & $\mathrm{A}$ & - & $\mathrm{B}$ & $\mathrm{D}$ & - \\
\hline 151 & 137 & M & 163462 & $\mathrm{D}$ & A & A & $\mathrm{C}$ & B & B & B & A & $\mathrm{C}$ & B & $\mathrm{D}$ & 742 \\
\hline 152 & 137 & M & 163463 & $\mathrm{D}$ & A & A & $\mathrm{C}$ & $\mathrm{B}$ & B & B & A & $\mathrm{C}$ & B & $\mathrm{D}$ & 711 \\
\hline 153 & 137 & $\mathrm{~F}$ & 163464 & $\mathrm{D}$ & A & A & $\mathrm{C}$ & $\mathrm{B}$ & B & B & A & $\mathrm{C}$ & B & $\mathrm{D}$ & 696 \\
\hline 154 & 137 & M & 163465 & $\mathrm{D}$ & A & A & $\mathrm{C}$ & B & B & B & A & $\mathrm{C}$ & B & $\mathrm{D}$ & 769 \\
\hline 155 & 137 & M & 163466 & $\mathrm{D}$ & A & A & $\mathrm{C}$ & B & B & B & A & $\mathrm{C}$ & B & $\mathrm{D}$ & 822 \\
\hline 156 & 137 & M & 165832 & $\mathrm{D}$ & A & A & $\mathrm{C}$ & B & B & B & - & $\mathrm{C}$ & B & $\mathrm{D}$ & 784 \\
\hline 157 & 137 & $\mathrm{~F}$ & 165833 & $\mathrm{D}$ & A & A & $\mathrm{C}$ & $\mathrm{B}$ & B & B & - & $\mathrm{C}$ & B & $\mathrm{D}$ & 705 \\
\hline 158 & 137 & M & 167064 & $\mathrm{D}$ & A & A & $\mathrm{C}$ & $\mathrm{B}$ & B & B & - & $\mathrm{C}$ & B & $\mathrm{D}$ & 764 \\
\hline 159 & 138 & $\mathrm{~F}$ & 167068 & $\mathrm{D}$ & A & A & $\mathrm{C}$ & B & B & $\mathrm{BD}$ & - & - & B & $\mathrm{D}$ & 788 \\
\hline 160) & 138 & M & 167069 & $\mathrm{D}$ & A & A & $\mathrm{C}$ & B & B & B & $\mathrm{AB}$ & B & B & $\mathrm{D}$ & 783 \\
\hline 161 & 140 & M & 169999 & $\mathrm{D}$ & A & A & $\mathrm{C}$ & $\mathrm{B}$ & B & B & - & B & B & $\mathrm{D}$ & FMC \\
\hline 162 & 156 & M & 160162 & $\mathrm{D}$ & A & A & $\mathrm{C}$ & $\mathrm{B}$ & B & - & - & $\mathrm{BC}$ & B & $\mathrm{D}$ & 706 \\
\hline 163 & 156 & M & 160163 & $\mathrm{D}$ & $\mathrm{AB}$ & A & $\mathrm{C}$ & $\mathrm{B}$ & B & 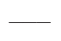 & - & $\mathrm{C}$ & B & $\mathrm{D}$ & 674 \\
\hline 164 & 156 & M & 160164 & $\mathrm{D}$ & A & A & $\mathrm{C}$ & B & B & 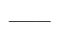 & - & $\mathrm{BC}$ & $\mathrm{B}$ & $\mathrm{D}$ & 639 \\
\hline 165 & 156 & M & 163366 & $\mathrm{D}$ & A & A & $\mathrm{C}$ & $\mathrm{B}$ & B & B & - & $\mathrm{C}$ & B & $\mathrm{D}$ & 688 \\
\hline 166 & 156 & M & 163367 & $\mathrm{D}$ & A & A & $\mathrm{C}$ & B & B & B & - & $\mathrm{BC}$ & B & $\mathrm{D}$ & 798 \\
\hline 167 & 156 & $\mathrm{~F}$ & 163369 & $\mathrm{D}$ & A & A & $\mathrm{C}$ & B & B & B & $\mathrm{AB}$ & B & B & $\mathrm{D}$ & 761 \\
\hline 168 & 157 & M & 163368 & $\mathrm{D}$ & $\mathrm{AC}$ & A & $\mathrm{C}$ & B & B & - & $\mathrm{AB}$ & $\mathrm{BC}$ & B & $\mathrm{D}$ & 832 \\
\hline 169 & 158 & M & 165834 & $\mathrm{D}$ & A & A & $\mathrm{C}$ & B & B & - & - & $\mathrm{BC}$ & B & $\mathrm{D}$ & 790 \\
\hline 170) & 158 & M & 165835 & $\mathrm{D}$ & A & A & $\mathrm{C}$ & $\mathrm{B}$ & B & B & - & $\mathrm{C}$ & B & $\mathrm{D}$ & 710 \\
\hline 171 & 158 & M & 165836 & $\mathrm{D}$ & A & A & - & B & $\mathrm{AB}$ & B & - & $\mathrm{BC}$ & B & $\mathrm{D}$ & 387 \\
\hline 172 & 158 & $\mathrm{~F}$ & 165837 & $\mathrm{D}$ & A & A & $\mathrm{AC}$ & B & $\mathrm{AB}$ & B & - & B & B & $\mathrm{D}$ & 476 \\
\hline 173 & 158 & M & 165838 & $\mathrm{D}$ & A & A & $\mathrm{C}$ & B & B & B & $\mathrm{AB}$ & B & B & $\mathrm{D}$ & - \\
\hline 174 & 158 & M & 165839 & $\mathrm{D}$ & A & A & $\mathrm{C}$ & B & B & B & - & B & B & $\mathrm{D}$ & 804 \\
\hline 175 & 158 & M & 165840 & $\mathrm{D}$ & A & A & $\mathrm{C}$ & B & B & B & - & $\mathrm{BC}$ & B & $\mathrm{D}$ & 823 \\
\hline 176 & 159 & M & 163370 & $\mathrm{D}$ & A & A & $\mathrm{C}$ & $\mathrm{B}$ & B & B & A & $\mathrm{BC}$ & B & $\mathrm{D}$ & 718 \\
\hline 177 & 159 & $\mathrm{~F}$ & 163371 & $\mathrm{D}$ & A & A & $\mathrm{C}$ & $\mathrm{B}$ & B & B & A & $\mathrm{C}$ & $\mathrm{B}$ & $\mathrm{D}$ & 766 \\
\hline 178 & 163 & M & 163361 & $\mathrm{D}$ & A & A & $\mathrm{C}$ & B & B & B & - & $\mathrm{BC}$ & B & $\mathrm{D}$ & 749 \\
\hline
\end{tabular}


Locus

Site Sex AMNH Aat-1 Aat-2 Idh-1 Ldh-1 Ldh-2 Mdh-1 Mpi Pgi Pgm-1 Pgm-2 Sod-1 blood

\begin{tabular}{|c|c|c|c|c|c|c|c|c|c|c|c|c|c|c|c|}
\hline 179 & 163 & M & 163362 & $\mathrm{D}$ & A & A & $\mathrm{C}$ & B & B & B & - & $\mathrm{C}$ & B & $\mathrm{D}$ & 712 \\
\hline 180) & 163 & $\mathrm{~F}$ & 163363 & $\mathrm{D}$ & A & A & $\mathrm{C}$ & B & B & B & A & $\mathrm{C}$ & B & $\mathrm{D}$ & 849 \\
\hline 181 & 164 & M & 160165 & $\mathrm{D}$ & A & A & $\mathrm{C}$ & B & B & B & A & $\mathrm{BC}$ & B & $\mathrm{D}$ & 785 \\
\hline 182 & 164 & $\mathrm{~F}$ & 162900 & $\mathrm{D}$ & B & A & $\mathrm{C}$ & B & 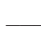 & 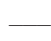 & A & - & 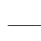 & $\mathrm{D}$ & 728 \\
\hline 183 & 164 & M & 163354 & $\mathrm{D}$ & A & A & $\mathrm{C}$ & B & B & B & $\mathrm{AB}$ & $\mathrm{BC}$ & B & $\mathrm{D}$ & 803 \\
\hline 184 & 164 & M & 163355 & $\mathrm{D}$ & A & A & $\mathrm{C}$ & B & B & B & - & $\mathrm{BC}$ & B & $\mathrm{D}$ & 781 \\
\hline 185 & 164 & M & 163356 & $\mathrm{D}$ & A & A & $\mathrm{C}$ & B & B & B & - & $\mathrm{C}$ & B & $\mathrm{D}$ & 810 \\
\hline 186 & 164 & M & 163357 & $\mathrm{D}$ & A & A & $\mathrm{C}$ & B & B & B & - & $\mathrm{BC}$ & B & $\mathrm{D}$ & 769 \\
\hline 187 & 166 & M & 163352 & $\mathrm{D}$ & A & A & $\mathrm{C}$ & B & B & B & A & $\mathrm{BC}$ & B & $\mathrm{D}$ & 907 \\
\hline 188 & 166 & M & 163353 & $\mathrm{D}$ & A & A & $\mathrm{C}$ & B & B & B & 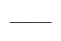 & $\mathrm{BC}$ & B & $\mathrm{D}$ & 830 \\
\hline 189 & 166 & $\mathrm{~F}$ & 163365 & $\mathrm{D}$ & A & A & $\mathrm{C}$ & B & B & 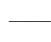 & A & $\mathrm{C}$ & $\mathrm{B}$ & $\mathrm{D}$ & 933 \\
\hline 190) & 167 & M & 163358 & $\mathrm{D}$ & A & A & $\mathrm{C}$ & B & B & B & A & B & B & $\mathrm{D}$ & 817 \\
\hline 191 & 167 & $\mathrm{~F}$ & 163359 & $\mathrm{D}$ & A & A & $\mathrm{C}$ & B & B & B & A & $\mathrm{BC}$ & B & $\mathrm{D}$ & 919 \\
\hline 192 & 167 & M & 163360 & $\mathrm{D}$ & $\mathrm{AC}$ & A & $\mathrm{C}$ & B & B & B & A & B & B & $\mathrm{D}$ & 837 \\
\hline 193 & 169 & M & 167005 & $\mathrm{D}$ & A & A & $\mathrm{C}$ & B & B & B & - & $\mathrm{BC}$ & B & $\mathrm{D}$ & 728 \\
\hline 194 & 170 & M & 160080 & $\mathrm{D}$ & A & A & $\mathrm{C}$ & B & B & B & - & B & B & $\mathrm{D}$ & 710 \\
\hline 195 & 170 & M & 160081 & $\mathrm{D}$ & A & A & $\mathrm{C}$ & B & B & B & - & B & B & $\mathrm{D}$ & 590 \\
\hline 196 & 170 & M & 160082 & $\mathrm{D}$ & A & A & $\mathrm{C}$ & B & B & B & - & B & B & $\mathrm{D}$ & 721 \\
\hline 197 & 170 & M & 160083 & $\mathrm{D}$ & A & A & $\mathrm{C}$ & B & B & B & A & $\mathrm{C}$ & B & $\mathrm{D}$ & 670 \\
\hline 198 & 170 & M & 160084 & $\mathrm{D}$ & A & A & $\mathrm{C}$ & B & B & B & A & $\mathrm{BC}$ & B & $\mathrm{D}$ & 756 \\
\hline 199 & 170 & M & 160085 & $\mathrm{D}$ & A & A & $\mathrm{C}$ & B & B & B & $\mathrm{AB}$ & B & B & $\mathrm{D}$ & 683 \\
\hline 200) & 170 & $\mathrm{~F}$ & 160086 & $\mathrm{D}$ & A & A & $\mathrm{C}$ & B & B & B & A & B & B & $\mathrm{D}$ & 628 \\
\hline 201 & 170 & M & 160087 & $\mathrm{~B}$ & A & A & $\mathrm{C}$ & B & B & B & - & B & B & $\mathrm{D}$ & 693 \\
\hline 202 & 170 & M & 160088 & $\mathrm{D}$ & A & A & $\mathrm{C}$ & B & B & B & - & B & $\mathrm{B}$ & $\mathrm{D}$ & 776 \\
\hline 203 & 170 & M & 160089 & $\mathrm{D}$ & A & A & $\mathrm{C}$ & B & B & B & - & B & B & $\mathrm{D}$ & 662 \\
\hline 204 & 170 & M & 160090 & $\mathrm{D}$ & A & A & $\mathrm{C}$ & B & B & B & - & $\mathrm{C}$ & B & $\mathrm{D}$ & 717 \\
\hline 205 & 170 & M & 160091 & $\mathrm{D}$ & A & A & $\mathrm{C}$ & B & B & B & - & $\mathrm{BC}$ & B & $\mathrm{D}$ & 738 \\
\hline 206 & 170 & M & 160092 & $\mathrm{D}$ & A & A & $\mathrm{C}$ & B & B & B & - & $\mathrm{C}$ & B & $\mathrm{D}$ & 681 \\
\hline 207 & 170 & M & 160093 & $\mathrm{D}$ & A & A & $\mathrm{C}$ & B & B & B & - & B & B & $\mathrm{D}$ & 734 \\
\hline 208 & 170 & M & 160094 & $\mathrm{D}$ & A & A & $\mathrm{C}$ & B & B & B & - & $\mathrm{BC}$ & B & $\mathrm{D}$ & 702 \\
\hline 209 & 170 & M & 160095 & $\mathrm{D}$ & A & A & $\mathrm{C}$ & B & B & 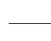 & - & B & B & $\mathrm{D}$ & 735 \\
\hline 210) & 170 & M & 160096 & $\mathrm{D}$ & A & A & $\mathrm{C}$ & B & B & 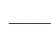 & A & $\mathrm{BC}$ & B & $\mathrm{D}$ & 769 \\
\hline 211 & 170 & M & 160097 & $\mathrm{D}$ & A & A & $\mathrm{C}$ & B & B & 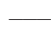 & A & B & B & $\mathrm{D}$ & 706 \\
\hline 212 & 170 & M & 160098 & $\mathrm{D}$ & A & A & $\mathrm{C}$ & B & B & B & QA & $\mathrm{BC}$ & B & $\mathrm{D}$ & 778 \\
\hline 213 & 170 & M & 160099 & $\mathrm{D}$ & A & A & $\mathrm{C}$ & B & B & B & A & $\mathrm{BC}$ & B & $\mathrm{D}$ & 663 \\
\hline 214 & 170 & $\mathrm{~F}$ & 160100 & $\mathrm{D}$ & A & A & $\mathrm{C}$ & B & B & B & - & $\mathrm{BC}$ & B & $\mathrm{D}$ & 794 \\
\hline 215 & 170 & M & 160101 & $\mathrm{D}$ & A & A & $\mathrm{C}$ & B & B & B & - & B & B & $\mathrm{D}$ & 799 \\
\hline 216 & 170 & $\mathrm{~F}$ & 160102 & $\mathrm{D}$ & A & A & $\mathrm{C}$ & B & B & - & A & B & B & $\mathrm{D}$ & 753 \\
\hline 217 & 170 & M & 160103 & $\mathrm{D}$ & A & A & $\mathrm{C}$ & B & B & 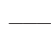 & 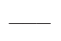 & B & B & $\mathrm{D}$ & 703 \\
\hline 218 & 170 & $\mathrm{~F}$ & 160104 & $\mathrm{D}$ & A & A & $\mathrm{C}$ & B & B & 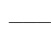 & 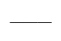 & B & B & $\mathrm{D}$ & 770 \\
\hline 219 & 170 & M & 160105 & $\mathrm{D}$ & A & A & $\mathrm{C}$ & B & B & 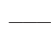 & - & B & B & $\mathrm{D}$ & 705 \\
\hline 220) & 170 & $\mathrm{~F}$ & 160106 & $\mathrm{D}$ & A & A & $\mathrm{C}$ & B & B & 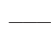 & A & B & B & $\mathrm{D}$ & 692 \\
\hline 221 & 170 & $\mathrm{~F}$ & 160107 & $\mathrm{D}$ & A & A & $\mathrm{C}$ & B & B & - & $\mathrm{AB}$ & B & B & $\mathrm{D}$ & 740 \\
\hline 222 & 170 & M & 160108 & $\mathrm{D}$ & A & A & $\mathrm{C}$ & B & B & 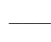 & A & $\mathrm{BC}$ & B & $\mathrm{D}$ & 793 \\
\hline 223 & 170 & $\mathrm{~F}$ & 160109 & $\mathrm{D}$ & A & A & $\mathrm{C}$ & B & B & 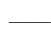 & A & B & B & $\mathrm{D}$ & 649 \\
\hline 224 & 170 & $\mathrm{~F}$ & 160110 & $\mathrm{D}$ & A & A & $\mathrm{C}$ & B & B & 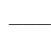 & A & $\mathrm{BC}$ & B & $\mathrm{D}$ & 658 \\
\hline 225 & 170 & M & 160111 & $\mathrm{D}$ & A & A & $\mathrm{C}$ & B & B & 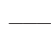 & - & B & B & $\mathrm{D}$ & 732 \\
\hline 226 & 170 & M & 160112 & $\mathrm{D}$ & A & A & $\mathrm{C}$ & B & B & 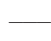 & - & $\mathrm{BC}$ & B & $\mathrm{D}$ & 722 \\
\hline 227 & 171 & M & 165841 & $\mathrm{D}$ & A & A & $\mathrm{C}$ & B & B & B & $\mathrm{AB}$ & B & B & $\mathrm{D}$ & 877 \\
\hline 228 & 171 & M & 165842 & $\mathrm{D}$ & A & A & $\mathrm{C}$ & B & B & - & A & B & B & $\mathrm{D}$ & 778 \\
\hline 229 & 171 & $\mathrm{~F}$ & 165843 & $\mathrm{D}$ & A & A & $\mathrm{C}$ & B & B & - & A & B & $\mathrm{B}$ & $\mathrm{D}$ & 733 \\
\hline 230) & 171 & M & 165844 & $\mathrm{D}$ & A & A & $\mathrm{C}$ & B & B & 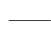 & A & B & B & $\mathrm{D}$ & 802 \\
\hline
\end{tabular}




\begin{tabular}{|c|c|c|c|c|c|c|c|c|c|c|c|c|c|c|c|}
\hline & \multirow[b]{2}{*}{ Site } & \multirow[b]{2}{*}{ Sex } & \multirow[b]{2}{*}{ AMNH } & \multicolumn{12}{|c|}{ Locus } \\
\hline & & & & Aat-1 & Aat-2 & Idh-1 & Ldh-1 & Ldh-2 & Mdh-1 & Mpi & Pgi & Pgm-1 & Pgm-2 & Sod-1 & blood \\
\hline 231 & 171 & M & 165845 & D & A & A & $\mathrm{C}$ & B & B & - & $\mathrm{AC}$ & - & B & D & 838 \\
\hline 232 & 171 & M & 165846 & D & A & A & $\mathrm{C}$ & B & B & B & A & $\mathrm{BC}$ & B & D & 795 \\
\hline 233 & 171 & M & 165847 & D & A & A & $\mathrm{C}$ & B & B & 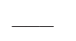 & A & B & B & $\mathrm{D}$ & 713 \\
\hline 234 & 171 & F & 165848 & D & A & A & C & B & B & - & $\mathrm{AB}$ & C & B & D & 699 \\
\hline 235 & 171 & M & 165849 & D & A & A & $\mathrm{C}$ & B & B & - & A & $\mathrm{BC}$ & B & D & 765 \\
\hline 236 & 171 & M & 165850 & D & A & A & $\mathrm{C}$ & B & B & - & $\mathrm{AC}$ & B & B & D & 788 \\
\hline 237 & 172 & M & 169483 & D & A & A & $\mathrm{C}$ & B & B & B & B & B & B & D & FCM \\
\hline 238 & 172 & M & 169484 & D & A & A & C & B & B & B & A & B & B & D & FCM \\
\hline 239 & 172 & M & 169485 & D & A & A & C & B & B & B & B & B & B & D & FCM \\
\hline 240) & 172 & M & 169486 & D & A & A & $\mathrm{C}$ & B & B & B & A & B & B & D & FCM \\
\hline 241 & 172 & M & 169487 & D & A & A & $\mathrm{C}$ & B & B & B & A & B & B & D & FCM \\
\hline 242 & 172 & $\mathrm{~F}$ & 169488 & D & A & A & $\mathrm{C}$ & B & B & B & $\mathrm{AB}$ & B & B & D & $\mathrm{FCM}$ \\
\hline 243 & 172 & F & 169489 & D & A & A & C & B & B & B & A & B & B & D & FCM \\
\hline 244 & 172 & F & 169490 & D & A & A & C & B & B & B & $\mathrm{AB}$ & B & B & D & FCM \\
\hline 245 & 172 & F & 169491 & D & A & A & C & B & B & B & A & B & B & D & FCM \\
\hline 246 & 173 & M & 169492 & D & A & A & $\mathrm{C}$ & B & B & B & A & B & B & D & $\mathrm{FCM}$ \\
\hline 247 & 173 & M & 169493 & D & A & A & C & B & B & B & A & B & B & D & FCM \\
\hline 248 & 173 & $\mathrm{M}$ & 169494 & D & A & A & C & B & B & B & A & B & B & D & FCM \\
\hline 249 & 173 & M & 169495 & D & A & A & C & B & B & B & A & B & B & D & FCM \\
\hline 250) & 173 & M & 169496 & D & A & A & C & B & B & B & A & C & B & D & FCM \\
\hline 251 & 173 & M & 169497 & D & A & A & $\mathrm{C}$ & B & B & B & A & 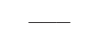 & B & D & FCM \\
\hline 252 & 173 & M & 169498 & D & A & A & $\mathrm{C}$ & B & B & B & - & B & B & D & FCM \\
\hline 253 & 173 & M & 169499 & D & A & A & $\mathrm{C}$ & B & B & B & A & B & B & D & FCM \\
\hline 254 & 173 & M & 169500 & D & A & A & C & B & B & B & A & B & B & D & FCM \\
\hline 255 & 173 & M & 169501 & D & A & A & $\mathrm{C}$ & B & B & B & A & B & B & D & FCM \\
\hline 256 & 173 & F & 169502 & D & A & A & C & B & B & B & A & B & B & D & FCM \\
\hline 257 & 173 & F & 169503 & D & A & A & C & B & B & B & $\mathrm{AC}$ & C & B & D & FCM \\
\hline 258 & 173 & F & 169504 & D & A & A & C & B & B & B & A & B & B & D & FCM \\
\hline 259 & 173 & F & 169505 & D & A & A & $\mathrm{C}$ & B & B & B & A & B & B & D & FCM \\
\hline 260) & 173 & F & 169506 & D & A & A & C & B & B & B & A & B & B & D & FCM \\
\hline 261 & 173 & F & 169929 & D & A & A & C & B & B & B & A & B & B & D & FCM \\
\hline 262 & 173 & F & 169930 & D & A & A & C & B & B & B & A & B & B & D & FCM \\
\hline 263 & 175 & M & 169462 & D & A & A & C & B & B & B & $\mathrm{AB}$ & $\mathrm{BC}$ & B & D & FCM \\
\hline 264 & 175 & M & 169463 & D & A & A & $\mathrm{C}$ & B & B & B & - & $\mathrm{BC}$ & B & D & FCM \\
\hline 265 & 175 & M & 169464 & D & A & A & C & B & B & B & B & $\mathrm{BC}$ & B & D & FCM \\
\hline 266 & 175 & M & 169465 & D & A & A & $\mathrm{C}$ & B & B & B & A & $\mathrm{BC}$ & B & D & FCM \\
\hline 267 & 175 & M & 169466 & D & A & A & C & B & B & B & $\mathrm{AB}$ & B & B & D & FCM \\
\hline 268 & 175 & M & 169467 & D & A & A & $\mathrm{C}$ & B & B & B & B & B & B & D & FCM \\
\hline 269 & 175 & M & 169468 & D & A & A & C & B & B & B & $\mathrm{AC}$ & B & B & D & FCM \\
\hline 270) & 175 & M & 169469 & D & A & A & C & B & B & B & $\mathrm{AB}$ & B & B & D & FCM \\
\hline 271 & 175 & M & 169470 & D & A & A & C & B & B & B & A & C & B & D & FCM \\
\hline 272 & 175 & M & 169471 & D & A & A & C & B & B & B & 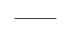 & $\mathrm{BC}$ & B & D & FCM \\
\hline 273 & 175 & M & 169472 & D & A & A & C & B & B & B & B & B & B & D & FCM \\
\hline 274 & 175 & M & 169473 & D & A & A & C & B & B & B & $\mathrm{AB}$ & C & B & D & FCM \\
\hline 275 & 175 & $\mathrm{M}$ & 169474 & D & A & A & C & B & B & B & B & C & B & D & FCM \\
\hline 276 & 175 & M & 169475 & D & A & A & C & B & B & B & B & C & B & D & FCM \\
\hline 277 & 175 & M & 169476 & D & A & A & C & B & B & B & B & B & B & D & FCM \\
\hline 278 & 175 & F & 169477 & D & A & A & C & B & B & B & B & B & B & D & FCM \\
\hline 279 & 175 & F & 169478 & D & A & A & $\mathrm{C}$ & B & B & B & B & $\mathrm{BC}$ & B & D & FCM \\
\hline 280) & 175 & F & 169479 & D & A & A & C & B & B & B & B & B & B & D & FCM \\
\hline 281 & 175 & $\mathrm{~F}$ & 169480 & D & A & A & $\mathrm{C}$ & B & B & B & B & $\mathrm{BC}$ & B & D & FCM \\
\hline 282 & 175 & F & 169481 & D & A & A & C & B & B & B & $\mathrm{AB}$ & B & B & D & FCM \\
\hline
\end{tabular}




\begin{tabular}{|c|c|c|c|c|c|c|c|c|c|c|c|c|c|c|c|}
\hline & \multirow[b]{2}{*}{ Site } & \multirow[b]{2}{*}{ Sex } & \multirow[b]{2}{*}{ AMNH } & \multicolumn{12}{|c|}{ Locus } \\
\hline & & & & Aat-1 & Aat-2 & Idh-1 & Ldh-1 & Ldh-2 & Mdh-1 & Mpi & Pgi & Pgm-1 & Pgm-2 & Sod-1 & blood \\
\hline 283 & 175 & $\mathrm{~F}$ & 169482 & $\mathrm{D}$ & A & A & C & B & B & B & $\mathrm{AB}$ & B & B & D & FCM \\
\hline 284 & 176 & $\mathrm{~F}$ & 163433 & D & A & A & C & B & B & B & $\mathrm{AB}$ & B & B & $\mathrm{D}$ & 794 \\
\hline 285 & 177 & M & 165851 & $\mathrm{D}$ & A & A & $\mathrm{C}$ & B & B & 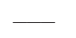 & $\mathrm{AB}$ & $\mathrm{C}$ & B & D & 725 \\
\hline 286 & 177 & M & 165852 & $\mathrm{D}$ & A & A & $\mathrm{C}$ & B & B & 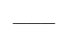 & A & $\mathrm{BC}$ & B & $\mathrm{D}$ & 837 \\
\hline 287 & 177 & M & 165853 & $\mathrm{D}$ & A & A & $\mathrm{C}$ & B & B & B & - & $\mathrm{C}$ & B & $\mathrm{D}$ & 754 \\
\hline 288 & 177 & M & 165854 & $\mathrm{D}$ & A & A & $\mathrm{C}$ & B & B & - & - & $\mathrm{C}$ & B & $\mathrm{D}$ & 761 \\
\hline 289 & 177 & M & 165855 & $\mathrm{D}$ & A & A & $\mathrm{C}$ & B & B & B & - & $\mathrm{C}$ & B & $\mathrm{D}$ & 722 \\
\hline 290) & 178 & F & 163434 & $\mathrm{D}$ & A & A & $\mathrm{C}$ & B & B & B & A & $\mathrm{C}$ & B & $\mathrm{D}$ & 891 \\
\hline 291 & 179 & M & 169507 & $\mathrm{D}$ & A & A & $\mathrm{C}$ & B & B & B & $\mathrm{AB}$ & $\mathrm{BC}$ & B & D & FCM \\
\hline 292 & 179 & $\mathrm{M}$ & 169508 & $\mathrm{D}$ & A & A & $\mathrm{C}$ & B & B & B & - & $\mathrm{BC}$ & B & $\mathrm{D}$ & FCM \\
\hline 293 & 179 & M & 169509 & D & A & A & $\mathrm{C}$ & B & B & B & - & $\mathrm{BC}$ & B & D & FCM \\
\hline 294 & 179 & $\mathrm{~F}$ & 169510 & $\mathrm{D}$ & A & A & $\mathrm{C}$ & B & B & B & A & $\mathrm{BC}$ & B & D & FCM \\
\hline 295 & 179 & $\mathrm{~F}$ & 169834 & $\mathrm{D}$ & A & A & $\mathrm{C}$ & B & B & B & B & B & B & D & FCM \\
\hline 296 & 179 & $\mathrm{~F}$ & 169835 & $\mathrm{D}$ & A & A & C & B & B & B & A & $\mathrm{BC}$ & B & D & FCM \\
\hline 297 & 179 & $\mathrm{~F}$ & 169836 & D & A & A & $\mathrm{C}$ & B & B & B & $\mathrm{AB}$ & $\mathrm{BC}$ & B & D & FCM \\
\hline 298 & 179 & F & 169837 & D & A & A & C & B & B & B & B & $\mathrm{BC}$ & B & D & FCM \\
\hline 299 & 179 & M & 169838 & D & A & A & $\mathrm{C}$ & B & B & B & - & B & B & D & - \\
\hline 300) & 180 & F & 160113 & D & A & A & C & B & B & - & - & C & B & D & 651 \\
\hline 301 & 180 & M & 160114 & D & A & A & $\mathrm{C}$ & B & B & - & - & $\mathrm{C}$ & B & D & 632 \\
\hline 302 & 180 & M & 160115 & D & A & A & C & B & B & - & B & C & B & D & 670 \\
\hline 303 & 180 & M & 160116 & D & A & A & $\mathrm{C}$ & B & B & - & $\mathrm{AB}$ & B & B & D & 756 \\
\hline 304 & 180 & M & 160117 & D & A & A & $\mathrm{C}$ & B & B & - & - & B & B & D & 774 \\
\hline 305 & 180 & F & 160118 & D & A & A & C & B & B & - & - & C & B & D & 705 \\
\hline 306 & 180 & M & 160119 & D & A & A & C & B & B & - & - & C & B & D & 690 \\
\hline 307 & 180 & M & 160120 & D & A & A & C & B & B & - & B & C & B & D & 715 \\
\hline 308 & 180 & M & 160121 & D & A & A & $\mathrm{C}$ & B & B & - & $\mathrm{AB}$ & B & B & D & 595 \\
\hline 309 & 180 & M & 160122 & D & A & A & C & B & B & - & - & B & B & D & 708 \\
\hline 310) & 180 & $\mathrm{~F}$ & 160123 & D & A & A & C & B & B & - & $\mathrm{AB}$ & C & B & D & 618 \\
\hline 311 & 180 & $\mathrm{M}$ & 160124 & D & A & A & C & B & B & - & A & C & B & D & 704 \\
\hline 312 & 180 & M & 160125 & D & A & A & C & B & B & - & A & $\mathrm{C}$ & B & D & 719 \\
\hline 313 & 180 & M & 160126 & D & A & A & C & B & B & - & $\mathrm{AB}$ & B & B & D & 663 \\
\hline 314 & 180 & M & 160127 & D & A & A & $\mathrm{C}$ & B & B & - & - & B & B & D & 743 \\
\hline 315 & 180 & F & 160128 & D & A & A & C & B & B & - & A & C & B & D & 691 \\
\hline 316 & 180 & M & 160129 & D & A & A & $\mathrm{C}$ & B & B & - & A & $\mathrm{C}$ & B & D & 705 \\
\hline 317 & 180 & M & 160130 & D & A & A & C & B & B & - & B & C & B & D & 743 \\
\hline 318 & 180 & $\mathrm{M}$ & 160131 & D & A & A & C & B & B & - & $\mathrm{AB}$ & $\mathrm{BC}$ & B & D & 635 \\
\hline 319 & 180 & M & 160132 & D & A & A & C & B & B & - & - & B & B & D & 686 \\
\hline 320) & 180 & F & 160133 & D & A & A & $\mathrm{C}$ & B & B & - & - & C & B & D & 709 \\
\hline 321 & 180 & M & 160134 & D & A & A & C & B & B & - & - & C & B & D & 740 \\
\hline 322 & 180 & M & 160135 & D & A & A & $\mathrm{C}$ & B & B & - & B & C & B & D & 714 \\
\hline 323 & 180 & M & 160136 & D & A & A & $\mathrm{C}$ & B & B & - & $\mathrm{AB}$ & B & B & D & 648 \\
\hline 324 & 180 & $\mathrm{M}$ & 160137 & D & A & A & C & B & B & - & - & B & B & D & 729 \\
\hline 325 & 181 & M & 160138 & D & A & A & C & B & B & 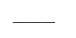 & - & $\mathrm{BC}$ & B & D & 861 \\
\hline 326 & 181 & M & 163390 & D & A & A & C & B & B & B & $\mathrm{AB}$ & $\mathrm{BC}$ & B & D & 846 \\
\hline 327 & 181 & $\mathrm{~F}$ & 163391 & D & A & A & $\mathrm{C}$ & B & B & B & - & C & B & D & 812 \\
\hline 328 & 181 & M & 163392 & D & A & A & C & B & B & B & A & B & B & D & 834 \\
\hline 329 & 181 & M & 163393 & D & A & A & $\mathrm{C}$ & B & B & B & - & C & B & D & 786 \\
\hline 330) & 181 & F & 163394 & D & A & A & C & B & B & B & - & C & B & D & 813 \\
\hline 331 & 181 & M & 163395 & D & A & A & C & B & B & B & - & B & B & D & 874 \\
\hline 332 & 181 & M & 163396 & D & A & A & $\mathrm{C}$ & B & B & - & $\mathrm{AB}$ & C & B & D & 802 \\
\hline 333 & 181 & F & 163397 & D & A & A & $\mathrm{C}$ & B & B & B & A & C & B & D & 820 \\
\hline 334 & 181 & M & 163398 & D & A & A & $\mathrm{C}$ & B & B & B & $\mathrm{AB}$ & C & B & D & 885 \\
\hline
\end{tabular}


APPENDIX 2-2

(Continued)

\begin{tabular}{|c|c|c|c|c|c|c|c|c|c|c|c|c|c|c|c|}
\hline & \multirow[b]{2}{*}{ Site } & \multirow[b]{2}{*}{ Sex } & \multirow[b]{2}{*}{ AMNH } & \multicolumn{12}{|c|}{ Locus } \\
\hline & & & & Aat-1 & Aat-2 & Idh-1 & Ldh-1 & Ldh-2 & Mdh-1 & Mpi & Pgi & Pgm-1 & Pgm-2 & Sod-1 & blood \\
\hline 335 & 181 & $\mathrm{~F}$ & 163399 & D & A & A & C & B & B & B & - & C & B & D & 759 \\
\hline 336 & 181 & $\mathrm{~F}$ & 163400 & D & A & A & C & B & B & B & A & $\mathrm{BC}$ & B & D & 830 \\
\hline 337 & 181 & $\mathrm{M}$ & 163401 & D & A & A & $\mathrm{C}$ & B & B & B & A & $\mathrm{BC}$ & B & $\mathrm{D}$ & 939 \\
\hline 338 & 181 & $\mathrm{~F}$ & 163402 & D & A & A & C & B & B & B & - & C & B & D & 836 \\
\hline 339 & 181 & M & 163403 & D & A & A & C & B & B & B & $\mathrm{AB}$ & B & B & $\mathrm{D}$ & - \\
\hline 340) & 181 & M & 163404 & D & A & A & C & B & B & B & A & C & B & $\mathrm{D}$ & 702 \\
\hline 341 & 182 & M & 163405 & D & A & A & C & B & B & B & B & $\mathrm{BC}$ & B & $\mathrm{D}$ & 801 \\
\hline 342 & 182 & M & 163406 & D & A & A & C & B & B & B & 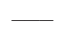 & B & B & $\mathrm{D}$ & 844 \\
\hline 343 & 182 & M & 163407 & D & A & A & C & B & B & B & B & $\mathrm{BC}$ & B & $\mathrm{D}$ & 728 \\
\hline 344 & 182 & M & 163408 & D & A & A & C & B & B & B & B & B & B & $\mathrm{D}$ & 792 \\
\hline 345 & 182 & $\mathrm{M}$ & 163409 & D & A & A & $\mathrm{C}$ & B & B & B & B & $\mathrm{BC}$ & B & $\mathrm{D}$ & 806 \\
\hline 346 & 182 & $\mathrm{M}$ & 163410 & $\mathrm{D}$ & A & A & $\mathrm{C}$ & B & B & B & A & $\mathrm{BC}$ & B & $\mathrm{D}$ & 753 \\
\hline 347 & 182 & $\mathrm{~F}$ & 163411 & D & A & A & C & B & B & B & - & B & B & D & 736 \\
\hline 348 & 182 & $\mathrm{M}$ & 163412 & D & A & A & C & B & B & - & B & B & B & $\mathrm{D}$ & 782 \\
\hline 349 & 182 & M & 163413 & D & A & A & C & B & B & B & B & $\mathrm{BC}$ & B & D & 796 \\
\hline 350) & 182 & M & 163414 & D & A & A & C & B & B & B & B & B & B & $\mathrm{D}$ & 805 \\
\hline 351 & 182 & M & 163415 & D & A & A & C & B & B & B & B & B & B & $\mathrm{D}$ & 877 \\
\hline 352 & 182 & M & 163416 & D & A & A & C & B & B & B & B & $\mathrm{BC}$ & B & D & 808 \\
\hline 353 & 182 & $\mathrm{~F}$ & 163417 & D & A & A & C & B & B & - & B & B & B & $\mathrm{D}$ & 791 \\
\hline 354 & 182 & $\mathrm{~F}$ & 163418 & D & A & A & C & B & B & B & $\mathrm{AB}$ & $\mathrm{BC}$ & B & D & 780 \\
\hline 355 & 182 & $\mathrm{~F}$ & 163419 & D & A & A & C & B & B & - & $\mathrm{AB}$ & B & B & $\mathrm{D}$ & 887 \\
\hline 356 & 182 & $\mathrm{~F}$ & 163420 & $\mathrm{D}$ & A & A & C & B & B & - & B & $\mathrm{BC}$ & B & $\mathrm{D}$ & 757 \\
\hline 357 & 182 & $\mathrm{~F}$ & 163421 & D & A & A & C & B & B & 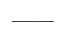 & B & B & B & $\mathrm{D}$ & 766 \\
\hline 358 & 182 & $\mathrm{~F}$ & 163422 & D & A & A & C & B & B & B & A & B & B & $\mathrm{D}$ & 812 \\
\hline 359 & 182 & $\mathrm{~F}$ & 163423 & D & A & A & C & B & B & B & B & $\mathrm{BC}$ & B & $\mathrm{D}$ & 768 \\
\hline 360) & 182 & $\mathrm{~F}$ & 163424 & D & A & A & C & B & B & B & B & $\mathrm{BD}$ & B & D & 728 \\
\hline 361 & 182 & $\mathrm{~F}$ & 163425 & D & A & A & C & B & B & B & B & $\mathrm{BC}$ & B & D & 811 \\
\hline 362 & 182 & M & 163426 & D & A & A & C & B & B & B & $\mathrm{AB}$ & $\mathrm{BD}$ & B & D & 712 \\
\hline 363 & 182 & $\mathrm{~F}$ & 163427 & D & A & A & C & B & B & B & - & BD & B & D & 699 \\
\hline 364 & 182 & $\mathrm{M}$ & 163428 & D & A & A & C & B & B & B & - & C & B & $\mathrm{D}$ & 746 \\
\hline 365 & 182 & M & 163429 & D & A & A & C & B & B & B & - & $\mathrm{BC}$ & B & D & 754 \\
\hline 366 & 182 & M & 163430 & D & A & A & C & B & B & B & - & $\mathrm{BC}$ & B & $\mathrm{D}$ & 859 \\
\hline 367 & 183 & $\mathrm{~F}$ & 163436 & D & A & A & C & B & B & B & $\mathrm{AB}$ & B & B & $\mathrm{D}$ & 714 \\
\hline 368 & 183 & $\mathrm{~F}$ & 163437 & D & A & A & C & B & B & B & B & B & B & $\mathrm{D}$ & 752 \\
\hline 369 & 183 & $\mathrm{~F}$ & 163438 & D & A & A & C & B & $\mathrm{BD}$ & B & B & B & B & $\mathrm{D}$ & 710 \\
\hline 370) & 183 & $\mathrm{M}$ & 163439 & D & A & A & C & B & B & B & B & B & B & $\mathrm{D}$ & 767 \\
\hline 371 & 183 & $\mathrm{M}$ & 163440 & D & A & A & C & B & B & B & B & B & B & D & 707 \\
\hline 372 & 183 & $\mathrm{~F}$ & 163441 & D & A & A & C & B & B & B & $\mathrm{AB}$ & B & B & $\mathrm{D}$ & 802 \\
\hline 373 & 183 & $?$ & 163442 & D & A & A & C & B & B & B & B & C & B & D & 694 \\
\hline 374 & 183 & $\mathrm{M}$ & 163443 & D & A & A & C & B & B & B & B & BC & B & D & 744 \\
\hline 375 & 183 & M & 163444 & D & A & A & C & B & B & B & $\mathrm{AB}$ & BD & B & $\mathrm{D}$ & 754 \\
\hline 376 & 183 & $?$ & 163445 & D & A & A & C & B & B & B & A & $\mathrm{BC}$ & B & D & 760 \\
\hline 377 & 183 & $\mathrm{~F}$ & 163446 & D & A & A & C & B & B & B & A & BD & B & D & 735 \\
\hline 378 & 183 & M & 163447 & D & A & A & C & B & B & B & $\mathrm{AB}$ & $\mathrm{BD}$ & B & D & 737 \\
\hline 379 & 183 & $\mathrm{~F}$ & 163448 & D & A & A & C & B & B & B & $\mathrm{AB}$ & B & B & D & 688 \\
\hline 380) & 183 & M & 163449 & D & A & A & C & B & BD & B & A & $\mathrm{BC}$ & B & $\mathrm{D}$ & 786 \\
\hline 381 & 183 & M? & 163450 & D & A & A & C & B & BD & B & $\mathrm{AB}$ & $\mathrm{BC}$ & B & $\mathrm{D}$ & 910 \\
\hline 382 & 183 & $\mathrm{~F}$ & 163451 & D & A & A & C & B & BD & B & A & C & B & D & 860 \\
\hline 383 & 184 & $\mathrm{M}$ & 163431 & D & A & A & C & B & B & B & - & C & B & $\mathrm{D}$ & 965 \\
\hline 384 & 184 & $\mathrm{M}$ & 163432 & D & A & A & C & B & B & B & $\mathrm{AB}$ & B & B & D & 728 \\
\hline 385 & 185 & $\mathrm{M}$ & 160139 & D & A & A & C & B & B & - & - & $\mathrm{BC}$ & B & D & 703 \\
\hline 386 & 185 & M & 160140 & D & A & A & C & B & B & B & B & B & B & D & 689 \\
\hline
\end{tabular}


Locus

Site Sex AMNH Aat-1 Aat-2 Idh-1 Ldh-1 Ldh-2 Mdh-1 Mpi Pgi Pgm-1 Pgm-2 Sod-1 blood

\begin{tabular}{|c|c|c|c|c|c|c|c|c|c|c|c|c|c|c|c|}
\hline 387 & 185 & M & 160141 & $\mathrm{D}$ & A & A & $\mathrm{C}$ & B & B & B & A & $\mathrm{C}$ & B & D & 616 \\
\hline 388 & 185 & M & 160142 & $\mathrm{D}$ & A & A & $\mathrm{C}$ & B & B & B & A & B & B & $\mathrm{D}$ & 686 \\
\hline 389 & 185 & M & 160143 & $\mathrm{D}$ & A & A & $\mathrm{C}$ & B & B & B & QA & B & B & $\mathrm{D}$ & 753 \\
\hline 390) & 185 & M & 167080 & $\mathrm{D}$ & A & A & $\mathrm{C}$ & B & B & B & - & B & B & $\mathrm{D}$ & 643 \\
\hline 391 & 185 & M & 167081 & $\mathrm{D}$ & A & A & $\mathrm{C}$ & B & B & B & - & $\mathrm{C}$ & B & $\mathrm{D}$ & 758 \\
\hline 392 & 185 & M & 167082 & $\mathrm{D}$ & A & A & $\mathrm{C}$ & B & B & B & - & $\mathrm{C}$ & B & $\mathrm{D}$ & 769 \\
\hline 393 & 185 & $\mathrm{~F}$ & 167083 & $\mathrm{D}$ & A & A & $\mathrm{C}$ & B & B & B & - & $\mathrm{C}$ & B & $\mathrm{D}$ & 770 \\
\hline 394 & 185 & M & 167084 & $\mathrm{D}$ & A & A & $\mathrm{C}$ & B & B & B & - & $\mathrm{BC}$ & B & $\mathrm{D}$ & 823 \\
\hline 395 & 185 & M & 167085 & $\mathrm{D}$ & A & A & $\mathrm{C}$ & B & B & B & - & B & B & $\mathrm{D}$ & 1063 \\
\hline 396 & 185 & M & 167086 & $\mathrm{D}$ & A & A & $\mathrm{C}$ & B & B & B & - & $\mathrm{C}$ & B & $\mathrm{D}$ & 765 \\
\hline 397 & 185 & M & 167087 & $\mathrm{D}$ & A & A & $\mathrm{C}$ & B & B & $\mathrm{B}$ & - & $\mathrm{C}$ & $\mathrm{B}$ & $\mathrm{D}$ & 671 \\
\hline 398 & 185 & M & 167088 & $\mathrm{D}$ & A & A & $\mathrm{C}$ & B & B & B & - & $\mathrm{BC}$ & B & $\mathrm{D}$ & 751 \\
\hline 399 & 185 & M & 167089 & $\mathrm{D}$ & A & A & $\mathrm{C}$ & B & B & B & - & $\mathrm{BC}$ & B & $\mathrm{D}$ & 736 \\
\hline 400) & 185 & $\mathrm{~F}$ & 167090 & $\mathrm{D}$ & A & A & $\mathrm{C}$ & B & B & B & B & $\mathrm{BC}$ & $\mathrm{B}$ & $\mathrm{D}$ & 760 \\
\hline 401 & 185 & $\mathrm{~F}$ & 167091 & $\mathrm{D}$ & A & A & $\mathrm{C}$ & B & B & B & - & $\mathrm{BC}$ & $\mathrm{B}$ & $\mathrm{D}$ & 842 \\
\hline 402 & 185 & $\mathrm{~F}$ & 167092 & $\mathrm{D}$ & A & A & $\mathrm{C}$ & B & B & B & A & $\mathrm{C}$ & B & $\mathrm{D}$ & 725 \\
\hline 403 & 186 & M & 160144 & $\mathrm{D}$ & A & A & $\mathrm{C}$ & B & B & B & $\mathrm{AB}$ & B & B & $\mathrm{D}$ & 672 \\
\hline 404 & 186 & $\mathrm{~F}$ & 160145 & $\mathrm{D}$ & A & A & $\mathrm{C}$ & B & B & 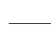 & A & - & 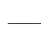 & $\mathrm{D}$ & 611 \\
\hline 405 & 186 & M & 160146 & $\mathrm{D}$ & A & A & $\mathrm{C}$ & B & B & B & $\mathrm{AB}$ & B & B & $\mathrm{D}$ & 624 \\
\hline 406 & 186 & M & 160147 & $\mathrm{D}$ & A & A & $\mathrm{C}$ & B & B & B & $\mathrm{AB}$ & B & B & $\mathrm{D}$ & 697 \\
\hline 407 & 186 & M & 160148 & $\mathrm{D}$ & A & A & $\mathrm{C}$ & B & B & B & A & $\mathrm{BC}$ & B & $\mathrm{D}$ & 658 \\
\hline 408 & 186 & M & 160149 & $\mathrm{D}$ & A & A & $\mathrm{C}$ & B & B & B & A & B & B & $\mathrm{D}$ & 614 \\
\hline 409 & 186 & M & 160150 & $\mathrm{D}$ & A & A & $\mathrm{C}$ & B & B & B & - & $\mathrm{C}$ & B & $\mathrm{D}$ & 633 \\
\hline 410) & 186 & M & 160151 & $\mathrm{D}$ & A & A & $\mathrm{C}$ & B & B & 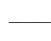 & $\mathrm{AC}$ & B & B & $\mathrm{D}$ & 664 \\
\hline 411 & 186 & M & 160152 & $\mathrm{D}$ & A & A & $\mathrm{C}$ & B & B & 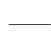 & A & $\mathrm{BC}$ & $\mathrm{B}$ & $\mathrm{D}$ & 708 \\
\hline 412 & 186 & $\mathrm{~F}$ & 160153 & $\mathrm{D}$ & A & A & $\mathrm{C}$ & B & B & B & A & B & B & $\mathrm{D}$ & 620 \\
\hline 413 & 186 & M & 160154 & $\mathrm{D}$ & A & A & $\mathrm{C}$ & B & B & B & $\mathrm{BC}$ & B & B & $\mathrm{D}$ & 630 \\
\hline 414 & 186 & M & 160155 & $\mathrm{D}$ & A & A & $\mathrm{C}$ & B & B & 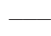 & A & $\mathrm{BC}$ & B & $\mathrm{D}$ & 650 \\
\hline 415 & 186 & M & 160156 & $\mathrm{D}$ & A & A & $\mathrm{C}$ & B & B & 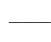 & A & B & B & $\mathrm{D}$ & 595 \\
\hline 416 & 186 & M & 160157 & $\mathrm{D}$ & A & A & $\mathrm{C}$ & B & B & - & - & $\mathrm{BC}$ & B & $\mathrm{D}$ & 690 \\
\hline 417 & 186 & $\mathrm{~F}$ & 167093 & $\mathrm{D}$ & A & A & $\mathrm{C}$ & B & B & B & - & $\mathrm{BC}$ & $\mathrm{B}$ & $\mathrm{D}$ & 722 \\
\hline 418 & 186 & $\mathrm{~F}$ & 167094 & $\mathrm{D}$ & A & A & $\mathrm{C}$ & B & B & B & - & $\mathrm{B}$ & B & $\mathrm{D}$ & 741 \\
\hline 419 & 186 & $\mathrm{~F}$ & 167095 & $\mathrm{D}$ & A & A & $\mathrm{C}$ & B & B & B & - & B & B & $\mathrm{D}$ & 693 \\
\hline 420) & 186 & $\mathrm{~F}$ & 167096 & $\mathrm{D}$ & A & A & $\mathrm{C}$ & B & B & B & - & $\mathrm{BC}$ & $\mathrm{B}$ & $\mathrm{D}$ & 1031 \\
\hline 431 & 187 & $\mathrm{~F}$ & 163452 & $\mathrm{D}$ & A & A & $\mathrm{C}$ & B & B & B & A & B & B & $\mathrm{D}$ & 784 \\
\hline 422 & 187 & $\mathrm{~F}$ & 163453 & $\mathrm{D}$ & A & A & $\mathrm{C}$ & B & B & B & A & B & B & $\mathrm{D}$ & 783 \\
\hline 423 & 187 & $\mathrm{~F}$ & 163454 & $\mathrm{D}$ & A & A & $\mathrm{C}$ & B & B & B & A & B & B & $\mathrm{D}$ & 731 \\
\hline 424 & 187 & $\mathrm{~F}$ & 163455 & $\mathrm{D}$ & A & A & $\mathrm{C}$ & B & B & B & A & B & $\mathrm{B}$ & $\mathrm{D}$ & 750 \\
\hline 425 & 187 & M & 163456 & $\mathrm{D}$ & A & A & $\mathrm{C}$ & B & B & B & A & B & B & $\mathrm{D}$ & 732 \\
\hline 426 & 187 & M & 167073 & $\mathrm{D}$ & A & A & $\mathrm{C}$ & B & B & B & - & B & B & $\mathrm{D}$ & 778 \\
\hline 427 & 187 & $\mathrm{~F}$ & 167074 & $\mathrm{D}$ & A & A & $\mathrm{C}$ & B & B & B & - & $\mathrm{C}$ & B & $\mathrm{D}$ & 643 \\
\hline 428 & 187 & M & 167075 & $\mathrm{D}$ & A & A & $\mathrm{C}$ & B & BD & B & 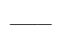 & B & B & $\mathrm{D}$ & 821 \\
\hline 429 & 187 & $\mathrm{~F}$ & 167076 & $\mathrm{D}$ & A & A & $\mathrm{C}$ & B & B & B & - & B & B & $\mathrm{D}$ & 768 \\
\hline 430) & 187 & $\mathrm{~F}$ & 167077 & $\mathrm{D}$ & A & A & $\mathrm{C}$ & B & B & B & - & B & B & $\mathrm{D}$ & 771 \\
\hline 431 & 187 & $\mathrm{~F}$ & 167078 & $\mathrm{D}$ & A & A & $\mathrm{C}$ & B & B & B & - & B & B & $\mathrm{D}$ & 763 \\
\hline 432 & 187 & M & 167079 & $\mathrm{D}$ & A & A & $\mathrm{C}$ & B & B & B & - & B & B & $\mathrm{D}$ & 770 \\
\hline 433 & 188 & M & 165856 & $\mathrm{D}$ & A & A & $\mathrm{C}$ & B & B & $\longrightarrow$ & $\mathrm{AC}$ & $\mathrm{BC}$ & B & $\mathrm{D}$ & 876 \\
\hline 434 & 188 & M & 165857 & $\mathrm{D}$ & A & A & $\mathrm{C}$ & B & B & 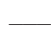 & $\mathrm{AB}$ & - & B & $\mathrm{D}$ & 749 \\
\hline 435 & 188 & M & 165858 & $\mathrm{D}$ & A & A & $\mathrm{C}$ & B & B & 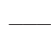 & $\mathrm{AC}$ & $\mathrm{BC}$ & B & $\mathrm{D}$ & 875 \\
\hline 436 & 188 & M & 165859 & $\mathrm{D}$ & A & A & $\mathrm{C}$ & B & B & 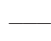 & $\mathrm{AB}$ & $\mathrm{BC}$ & B & $\mathrm{D}$ & 650 \\
\hline 437 & 188 & M & 165860 & $\mathrm{D}$ & A & A & $\mathrm{C}$ & B & B & 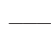 & $\mathrm{AB}$ & $\mathrm{B}$ & $\mathrm{B}$ & $\mathrm{D}$ & 635 \\
\hline 438 & 188 & M & 165861 & $\mathrm{D}$ & A & A & $\mathrm{C}$ & B & B & 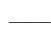 & $\mathrm{AC}$ & $\mathrm{BC}$ & B & $\mathrm{D}$ & 694 \\
\hline
\end{tabular}


APPENDIX 2-2

(Continued)

\begin{tabular}{|c|c|c|c|c|c|c|c|c|c|c|c|c|c|c|c|}
\hline & \multirow[b]{2}{*}{ Site } & \multirow[b]{2}{*}{ Sex } & \multirow[b]{2}{*}{ AMNH } & \multicolumn{12}{|c|}{ Locus } \\
\hline & & & & Aat-1 & Aat-2 & Idh-1 & Ldh-1 & Ldh-2 & Mdh-1 & Mpi & Pgi & Pgm-1 & Pgm-2 & Sod-1 & blood \\
\hline 439 & 188 & M & 165862 & D & A & A & $\mathrm{C}$ & B & B & - & A & - & B & D & 681 \\
\hline 440) & 188 & M & 165863 & D & A & A & $\mathrm{C}$ & B & B & - & $\mathrm{AB}$ & $\mathrm{BC}$ & B & D & 758 \\
\hline 441 & 188 & M & 165864 & D & A & A & $\mathrm{C}$ & B & B & - & A & $\mathrm{BC}$ & B & $\mathrm{D}$ & 771 \\
\hline 442 & 188 & M & 165865 & D & A & A & $\mathrm{C}$ & B & B & B & - & $\mathrm{C}$ & B & D & 708 \\
\hline 443 & 190 & M & JPB25772 & $\mathrm{D}$ & A & A & $\mathrm{C}$ & B & B & - & $\mathrm{AB}$ & $\mathrm{C}$ & B & $\mathrm{D}$ & - \\
\hline 444 & 190 & M & JPB25773 & D & A & A & $\mathrm{C}$ & B & B & - & A & B & B & $\mathrm{D}$ & 770 \\
\hline 445 & 190 & M & JPB25774 & $\mathrm{D}$ & A & A & $\mathrm{C}$ & B & B & - & $\mathrm{AB}$ & B & B & $\mathrm{D}$ & 648 \\
\hline 446 & 190 & M & JPB25775 & $\mathrm{D}$ & A & A & $\mathrm{C}$ & B & B & - & A & $\mathrm{BC}$ & B & $\mathrm{D}$ & 826 \\
\hline 447 & 191 & M & 163457 & $\mathrm{D}$ & A & A & $\mathrm{C}$ & B & B & - & A & $\mathrm{BC}$ & B & $\mathrm{D}$ & 831 \\
\hline 448 & 191 & M & 163459 & D & A & A & $\mathrm{C}$ & B & B & B & B & $\mathrm{BC}$ & B & D & 880 \\
\hline 449 & 191 & M & 163460 & D & A & A & $\mathrm{C}$ & B & B & B & A & B & B & D & 838 \\
\hline 450) & 192 & M & 160158 & $\mathrm{D}$ & A & A & $\mathrm{C}$ & B & B & B & - & $\mathrm{BC}$ & B & D & 904 \\
\hline 451 & 194 & M & 165866 & D & A & A & $\mathrm{C}$ & B & B & - & A & B & B & D & 711 \\
\hline 452 & 195 & M & 158779 & D & A & A & C & B & B & B & - & B & B & D & 808 \\
\hline 453 & 195 & M & 158780 & D & A & A & C & B & B & B & - & B & B & D & 763 \\
\hline 454 & 195 & $\mathrm{~F}$ & 158781 & D & A & A & C & B & B & B & - & B & B & D & 724 \\
\hline 455 & 195 & $\mathrm{~F}$ & 158782 & D & A & A & $\mathrm{C}$ & B & B & B & - & B & B & D & 764 \\
\hline 456 & 195 & M & 158783 & D & A & A & C & B & B & B & - & B & B & D & 757 \\
\hline 457 & 195 & M & 158784 & D & A & A & C & B & B & B & - & B & B & D & 783 \\
\hline 458 & 196 & M & 160159 & D & A & A & C & B & B & B & - & B & B & D & 785 \\
\hline 459 & 196 & F & 160160 & D & A & A & C & B & B & B & $\mathrm{AB}$ & B & B & D & 721 \\
\hline 460) & 196 & F & 160161 & $\mathrm{D}$ & A & A & C & B & B & B & - & B & B & D & 743 \\
\hline 461 & 203 & M & 165867 & D & A & A & C & B & B & 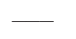 & A & B & B & D & 746 \\
\hline 462 & 205 & $\mathrm{~F}$ & 163373 & D & A & QA & C & B & B & B & A & $\ldots$ & B & D & 826 \\
\hline 463 & 212 & M & 163374 & D & A & A & C & B & B & $\mathrm{BC}$ & A & B & B & D & 728 \\
\hline 464 & 212 & F & 163375 & D & A & A & $\mathrm{C}$ & B & B & B & B & - & - & D & 732 \\
\hline
\end{tabular}

Appendix 2-3: Genotypes of diploid (2n) Ambystoma laterale-jeffersonianum LJ unisexual specimens ordered by site number.

\begin{tabular}{lclllllllllllllll}
\hline \hline & & & & \multicolumn{10}{c}{ Locus } \\
\cline { 6 - 16 } & Site & Sex & AMNH & Aat-1 & Aat-2 & Idh-1 & Ldh-1 & Ldh-2 & Mdh-1 & Mpi & Pgi & Pgm-1 & Pgm-2 & Sod-1 & blood \\
\hline 1 & 18 & F & 165937 & BD & AB & AB & BC & B & BD & BC & - & B & B & BD & 765 \\
2 & 107 & F & 169913 & BD & AB & AB & BC & A & BD & - & BC & B & B & BD & FCM \\
3 & 107 & F & 169914 & BD & AB & AB & BC & B & BD & - & BC & B & B & BD & FCM \\
4 & 107 & F & 169915 & BD & AB & AB & BC & A & BD & - & BC & BC & B & BD & FCM \\
5 & 107 & F & 169916 & BD & AB & AB & BC & A & BD & - & AC & B & B & BD & FCM \\
6 & 108 & F & 160248 & BD & AB & AB & BC & B & BD & AB & AC & B & B & BD & 752 \\
7 & 108 & F & 160249 & BD & AB & AB & BC & B & BD & AB & A & BC & B & BD & 836 \\
8 & 108 & F & 160250 & BD & AB & AB & BC & B & BD & - & AC & B & B & BD & 768 \\
9 & 108 & F & 160251 & BD & AB & AB & BC & B & BD & AB & AC & B & B & BD & 860 \\
$10)$ & 108 & F & 160252 & BD & AB & AB & BC & B & BD & - & AC & BC & B & BD & 755 \\
11 & 108 & F & 160253 & BD & AB & AB & BC & A & BD & - & AC & B & B & BD & 760 \\
12 & 108 & F & 160254 & BD & AB & AB & BC & B & BD & - & AC & B & B & BD & 707 \\
13 & 109 & F & 169917 & BD & AB & AB & BC & B & BD & - & AC & B & B & BD & FCM \\
14 & 109 & F & 169918 & BD & AB & AB & BC & B & BD & - & AC & B & B & BD & FCM \\
15 & 110 & F & 169395 & BD & AB & AB & BC & B & BD & AB & BC & - & B & BD & FCM \\
16 & 112 & F & 169802 & BD & AB & B & BC & B & BD & BD & AC & B & B & BD & FCM
\end{tabular}


APPENDIX 2-3

(Continued)

Locus

Site Sex AMNH Aat-1 Aat-2 Idh-1 Ldh-1 Ldh-2 Mdh-1 Mpi Pgi Pgm-1 Pgm-2 Sod-1 blood

\begin{tabular}{|c|c|c|c|c|c|c|c|c|c|c|c|c|c|c|c|}
\hline 17 & 113 & $\mathrm{~F}$ & 162819 & $\mathrm{BD}$ & $\mathrm{AB}$ & $\mathrm{AB}$ & $\mathrm{BC}$ & B & $\mathrm{BD}$ & $\mathrm{BC}$ & $\mathrm{BC}$ & B & B & $\mathrm{BD}$ & 787 \\
\hline 18 & 113 & $\mathrm{~F}$ & 162820 & $\mathrm{BD}$ & $\mathrm{AB}$ & $\mathrm{AB}$ & $\mathrm{BC}$ & B & $\mathrm{BD}$ & $\mathrm{BC}$ & $\mathrm{AC}$ & B & B & $\mathrm{BD}$ & 696 \\
\hline 19 & 113 & $\mathrm{~F}$ & 162821 & $\mathrm{BD}$ & $\mathrm{AB}$ & $\mathrm{AB}$ & $\mathrm{BC}$ & B & $\mathrm{BD}$ & $\mathrm{BC}$ & $\mathrm{C}$ & $\mathrm{BC}$ & B & $\mathrm{BD}$ & 726 \\
\hline 20) & 113 & $\mathrm{~F}$ & 162823 & $\mathrm{BD}$ & $\mathrm{AB}$ & $\mathrm{AB}$ & $\mathrm{BC}$ & B & $\mathrm{BD}$ & $\mathrm{BC}$ & $\mathrm{C}$ & $\mathrm{BC}$ & B & $\mathrm{BD}$ & 782 \\
\hline 21 & 113 & $\mathrm{~F}$ & 162824 & $\mathrm{BD}$ & $\mathrm{AB}$ & $\mathrm{AB}$ & $\mathrm{BC}$ & B & $\mathrm{BD}$ & $\mathrm{BC}$ & $\mathrm{C}$ & $\mathrm{BC}$ & B & BD & 860 \\
\hline 22 & 113 & $\mathrm{~F}$ & 162825 & $\mathrm{BD}$ & $\mathrm{AB}$ & $\mathrm{AB}$ & $\mathrm{BC}$ & B & $\mathrm{BD}$ & - & $\mathrm{AC}$ & B & $\mathrm{B}$ & $\mathrm{BD}$ & - \\
\hline 23 & 113 & $\mathrm{~F}$ & 162826 & $\mathrm{BD}$ & $\mathrm{AB}$ & $\mathrm{AB}$ & $\mathrm{BC}$ & B & $\mathrm{BD}$ & $\mathrm{BC}$ & - & B & B & $\mathrm{BD}$ & 893 \\
\hline 24 & 113 & $\mathrm{~F}$ & 162827 & $\mathrm{BD}$ & $\mathrm{AB}$ & $\mathrm{AB}$ & $\mathrm{BC}$ & B & $\mathrm{BD}$ & $\mathrm{BC}$ & - & B & B & $\mathrm{BD}$ & 760 \\
\hline 25 & 113 & $\mathrm{~F}$ & 162828 & $\mathrm{BD}$ & $\mathrm{AB}$ & $\mathrm{AB}$ & $\mathrm{BC}$ & B & $\mathrm{BD}$ & $\mathrm{BC}$ & - & B & B & $\mathrm{BD}$ & 789 \\
\hline 26 & 113 & $\mathrm{~F}$ & 162829 & $\mathrm{BD}$ & $\mathrm{AB}$ & $\mathrm{AB}$ & $\mathrm{BC}$ & B & $\mathrm{BD}$ & $\mathrm{BC}$ & - & B & B & $\mathrm{BD}$ & 797 \\
\hline 27 & 113 & $\mathrm{~F}$ & 162830 & BD & $\mathrm{AB}$ & $\mathrm{AB}$ & $\mathrm{BC}$ & B & $\mathrm{BD}$ & $\mathrm{BC}$ & $\mathrm{AC}$ & B & B & BD & 868 \\
\hline 28 & 113 & $\mathrm{~F}$ & 162862 & $\mathrm{BD}$ & $\mathrm{AB}$ & $\mathrm{AB}$ & $\mathrm{BC}$ & B & $\mathrm{BD}$ & - & - & $\mathrm{BC}$ & B & $\mathrm{BD}$ & 802 \\
\hline 29 & 113 & $\mathrm{~F}$ & 164590 & $\mathrm{BD}$ & $\mathrm{AB}$ & $\mathrm{AB}$ & $\mathrm{BC}$ & B & $\mathrm{BD}$ & - & - & $\mathrm{BC}$ & B & D & 759 \\
\hline 30) & 113 & $\mathrm{~F}$ & 164591 & $\mathrm{BD}$ & $\mathrm{AB}$ & $\mathrm{AB}$ & $\mathrm{BC}$ & B & $\mathrm{BD}$ & $\mathrm{BC}$ & $\mathrm{C}$ & $\mathrm{BC}$ & B & BD & 718 \\
\hline 31 & 113 & $\mathrm{~F}$ & 164592 & $\mathrm{BD}$ & $\mathrm{AB}$ & $\mathrm{AB}$ & $\mathrm{BC}$ & B & $\mathrm{BD}$ & $\mathrm{BC}$ & $\mathrm{AC}$ & B & B & $\mathrm{BD}$ & 642 \\
\hline 32 & 113 & $\mathrm{~F}$ & 164636 & $\mathrm{BD}$ & $\mathrm{AB}$ & $\mathrm{AB}$ & $\mathrm{BC}$ & B & $\mathrm{BD}$ & $\mathrm{BC}$ & $\mathrm{AC}$ & $\mathrm{BC}$ & B & $\mathrm{BD}$ & 978 \\
\hline 33 & 113 & $\mathrm{~F}$ & 164637 & $\mathrm{BD}$ & $\mathrm{AB}$ & $\mathrm{AB}$ & $\mathrm{BC}$ & B & $\mathrm{BD}$ & $\mathrm{BC}$ & $\mathrm{AC}$ & $\mathrm{BC}$ & B & $\mathrm{BD}$ & 758 \\
\hline 34 & 113 & $\mathrm{~F}$ & 164638 & $\mathrm{BD}$ & $\mathrm{AB}$ & $\mathrm{AB}$ & $\mathrm{BC}$ & B & $\mathrm{BD}$ & $\mathrm{BC}$ & $\mathrm{C}$ & $\mathrm{BC}$ & B & $\mathrm{BD}$ & 836 \\
\hline 35 & 114 & M & 153110 & $\mathrm{BD}$ & $\mathrm{AB}$ & A & $\mathrm{C}$ & B & $\mathrm{BD}$ & $\mathrm{BC}$ & - & B & $\mathrm{D}$ & $\mathrm{BD}$ & 708 \\
\hline 36 & 122 & $\mathrm{~F}$ & 153107 & $\mathrm{BD}$ & $\mathrm{AB}$ & $\mathrm{AB}$ & $\mathrm{BC}$ & 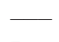 & $\mathrm{BD}$ & B & - & $\mathrm{C}$ & B & D & 766 \\
\hline 37 & 122 & M & 153108 & $\mathrm{BD}$ & $\mathrm{AB}$ & $\mathrm{AB}$ & $\mathrm{BC}$ & B & $\mathrm{BD}$ & - & $\mathrm{BC}$ & $\mathrm{BC}$ & B & BD & 736 \\
\hline 38 & 122 & $\mathrm{~F}$ & 153109 & $\mathrm{BD}$ & $\mathrm{AB}$ & $\mathrm{AB}$ & $\mathrm{BC}$ & B & $\mathrm{BD}$ & - & - & B & $\mathrm{B}$ & $\mathrm{BD}$ & 740 \\
\hline 39 & 122 & $\mathrm{~F}$ & 153119 & $\mathrm{BD}$ & $\mathrm{AB}$ & $\mathrm{AB}$ & $\mathrm{BC}$ & 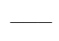 & $\mathrm{BD}$ & $\mathrm{AB}$ & - & B & B & $\mathrm{BD}$ & 823 \\
\hline 40) & 123 & $\mathrm{~F}$ & 153111 & $\mathrm{BD}$ & $\mathrm{AB}$ & $\mathrm{AB}$ & $\mathrm{BC}$ & 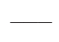 & $\mathrm{BD}$ & - & - & $\mathrm{BC}$ & B & $\mathrm{BD}$ & 702 \\
\hline 41 & 123 & $\mathrm{~F}$ & 162832 & $\mathrm{BD}$ & $\mathrm{AB}$ & $\mathrm{AB}$ & $\mathrm{BC}$ & B & $\mathrm{BD}$ & - & - & B & B & $\mathrm{BD}$ & 689 \\
\hline 42 & 123 & $\mathrm{~F}$ & 162833 & $\mathrm{BD}$ & $\mathrm{AB}$ & $\mathrm{AB}$ & B & B & $\mathrm{BD}$ & - & - & B & B & $\mathrm{BD}$ & 711 \\
\hline 43 & 123 & $\mathrm{~F}$ & 162834 & $\mathrm{BD}$ & $\mathrm{AB}$ & $\mathrm{AB}$ & $\mathrm{BC}$ & B & $\mathrm{BD}$ & - & - & B & B & $\mathrm{BD}$ & 690 \\
\hline 44 & 123 & $\mathrm{~F}$ & 165938 & $\mathrm{BD}$ & $\mathrm{AB}$ & $\mathrm{AB}$ & $\mathrm{BC}$ & B & $\mathrm{BD}$ & - & - & B & B & BD & - \\
\hline 45 & 123 & $\mathrm{~F}$ & 165939 & $\mathrm{BD}$ & $\mathrm{AB}$ & $\mathrm{AB}$ & $\mathrm{BC}$ & B & $\mathrm{BD}$ & - & - & B & B & $\mathrm{BD}$ & 612 \\
\hline 46 & 123 & $\mathrm{~F}$ & 165940 & $\mathrm{BD}$ & $\mathrm{AB}$ & $\mathrm{AB}$ & $\mathrm{BC}$ & B & $\mathrm{BD}$ & $\mathrm{BC}$ & - & B & B & $\mathrm{BD}$ & 572 \\
\hline 47 & 123 & $\mathrm{~F}$ & 165941 & $\mathrm{BD}$ & $\mathrm{AB}$ & $\mathrm{AB}$ & $\mathrm{BC}$ & B & $\mathrm{BD}$ & - & - & B & B & $\mathrm{BD}$ & 615 \\
\hline 48 & 123 & $\mathrm{~F}$ & 165942 & $\mathrm{BD}$ & $\mathrm{AB}$ & $\mathrm{AB}$ & B & B & $\mathrm{BD}$ & $\mathrm{BC}$ & - & B & B & $\mathrm{BD}$ & 647 \\
\hline 49 & 123 & $\mathrm{~F}$ & 165943 & $\mathrm{BD}$ & $\mathrm{AB}$ & $\mathrm{AB}$ & $\mathrm{BC}$ & B & $\mathrm{BD}$ & - & - & B & B & $\mathrm{BD}$ & 602 \\
\hline 50) & 123 & $\mathrm{~F}$ & 165944 & $\mathrm{BD}$ & $\mathrm{AB}$ & $\mathrm{AB}$ & $\mathrm{BC}$ & B & $\mathrm{BD}$ & $\mathrm{BC}$ & - & B & B & $\mathrm{BD}$ & 649 \\
\hline 51 & 124 & $\mathrm{~F}$ & 169810 & $\mathrm{BD}$ & $\mathrm{AB}$ & $\mathrm{AB}$ & $\mathrm{BC}$ & B & $\mathrm{BD}$ & $\mathrm{AB}$ & - & B & B & BD & FCM \\
\hline 52 & 124 & $\mathrm{~F}$ & 169811 & $\mathrm{BD}$ & $\mathrm{AB}$ & $\mathrm{AB}$ & $\mathrm{BC}$ & $\mathrm{AB}$ & $\mathrm{BD}$ & $\mathrm{AB}$ & $\mathrm{AC}$ & $\mathrm{BC}$ & B & $\mathrm{BD}$ & $\mathrm{FCM}$ \\
\hline 53 & 124 & $\mathrm{~F}$ & 169812 & $\mathrm{BD}$ & $\mathrm{AB}$ & $\mathrm{AB}$ & $\mathrm{BC}$ & B & $\mathrm{BD}$ & $\mathrm{BC}$ & $\mathrm{AC}$ & $\mathrm{BC}$ & B & $\mathrm{BD}$ & $\mathrm{FCM}$ \\
\hline 54 & 125 & $\mathrm{~F}$ & 169813 & $\mathrm{BD}$ & $\mathrm{AB}$ & $\mathrm{AB}$ & $\mathrm{BC}$ & $\mathrm{AB}$ & $\mathrm{BD}$ & $\mathrm{BC}$ & $\mathrm{AC}$ & $\mathrm{BC}$ & B & $\mathrm{BD}$ & $\mathrm{FCM}$ \\
\hline 55 & 125 & $\mathrm{~F}$ & 169814 & $\mathrm{BD}$ & $\mathrm{AB}$ & $\mathrm{AB}$ & $\mathrm{BC}$ & B & $\mathrm{BD}$ & $\mathrm{BC}$ & $\mathrm{AC}$ & $\mathrm{BC}$ & B & $\mathrm{BD}$ & $\mathrm{FCM}$ \\
\hline 56 & 126 & $\mathrm{~F}$ & 169772 & $\mathrm{BD}$ & $\mathrm{AB}$ & $\mathrm{AB}$ & $\mathrm{BC}$ & B & $\mathrm{BD}$ & $\mathrm{BC}$ & - & $\mathrm{BC}$ & B & $\mathrm{BD}$ & $\mathrm{FCM}$ \\
\hline 57 & 126 & $\mathrm{~F}$ & 169773 & $\mathrm{BD}$ & $\mathrm{AB}$ & $\mathrm{AB}$ & $\mathrm{BC}$ & B & $\mathrm{BD}$ & $\mathrm{BC}$ & - & $\mathrm{BC}$ & B & $\mathrm{BD}$ & $\mathrm{FCM}$ \\
\hline 58 & 126 & $\mathrm{~F}$ & 169774 & $\mathrm{BD}$ & $\mathrm{AB}$ & $\mathrm{AB}$ & $\mathrm{BC}$ & B & $\mathrm{BD}$ & $\mathrm{BC}$ & - & $\mathrm{BC}$ & B & $\mathrm{BD}$ & $\mathrm{FCM}$ \\
\hline 59 & 126 & $\mathrm{~F}$ & 169775 & $\mathrm{BD}$ & $\mathrm{AB}$ & $\mathrm{AB}$ & $\mathrm{BC}$ & B & $\mathrm{BD}$ & BD & - & $\mathrm{BC}$ & B & BD & $\mathrm{FCM}$ \\
\hline 60) & 126 & $\mathrm{~F}$ & 169776 & $\mathrm{BD}$ & $\mathrm{AB}$ & $\mathrm{AB}$ & $\mathrm{BC}$ & B & $\mathrm{BD}$ & $\mathrm{BC}$ & $\mathrm{AC}$ & $\mathrm{BC}$ & B & BD & FCM \\
\hline 61 & 130 & $\mathrm{~F}$ & 165945 & $\mathrm{BD}$ & $\mathrm{AB}$ & QB & $\mathrm{BC}$ & B & $\mathrm{BD}$ & $\mathrm{BC}$ & - & $\mathrm{BC}$ & $\mathrm{B}$ & $\mathrm{BD}$ & 816 \\
\hline 62 & 134 & $\mathrm{~F}$ & 162831 & $\mathrm{BD}$ & $\mathrm{AB}$ & $\mathrm{AB}$ & $\mathrm{BC}$ & B & $\mathrm{BD}$ & - & - & B & $\mathrm{B}$ & $\mathrm{BD}$ & 917 \\
\hline 63 & 137 & $\mathrm{~F}$ & 167065 & $\mathrm{BD}$ & $\mathrm{AB}$ & $\mathrm{AB}$ & $\mathrm{BC}$ & B & $\mathrm{BD}$ & $\mathrm{BC}$ & - & B & B & $\mathrm{BD}$ & 661 \\
\hline 64 & 137 & $\mathrm{~F}$ & 167066 & $\mathrm{BD}$ & $\mathrm{AB}$ & $\mathrm{AB}$ & - & B & - & $\mathrm{BC}$ & $\mathrm{AC}$ & B & B & $\mathrm{BD}$ & - \\
\hline 65 & 138 & $\mathrm{~F}$ & 167070 & $\mathrm{BD}$ & $\mathrm{AB}$ & $\mathrm{AB}$ & $\mathrm{BC}$ & B & - & $\mathrm{BC}$ & - & B & B & $\mathrm{BD}$ & 771 \\
\hline 66 & 156 & $\mathrm{~F}$ & 162816 & $\mathrm{BD}$ & $\mathrm{AB}$ & $\mathrm{AB}$ & $\mathrm{BC}$ & B & $\mathrm{BD}$ & $\mathrm{BC}$ & $\mathrm{AC}$ & $\mathrm{BC}$ & B & $\mathrm{BD}$ & 778 \\
\hline 67 & 156 & $\mathrm{~F}$ & 162817 & $\mathrm{BD}$ & $\mathrm{AB}$ & $\mathrm{AB}$ & $\mathrm{BC}$ & B & $\mathrm{BD}$ & $\mathrm{BC}$ & $\mathrm{AC}$ & $\mathrm{BC}$ & B & BD & 778 \\
\hline 68 & 156 & $\mathrm{~F}$ & 162818 & $\mathrm{BD}$ & $\mathrm{AB}$ & $\mathrm{AB}$ & $\mathrm{BC}$ & B & $\mathrm{BD}$ & $\mathrm{BC}$ & $\mathrm{AC}$ & $\mathrm{BC}$ & B & $\mathrm{BD}$ & 670 \\
\hline
\end{tabular}


APPENDIX 2-3

(Continued)

\begin{tabular}{|c|c|c|c|c|c|c|c|c|c|c|c|c|c|c|c|}
\hline & \multirow[b]{2}{*}{ Site } & \multirow[b]{2}{*}{ Sex } & \multirow[b]{2}{*}{ AMNH } & \multicolumn{12}{|c|}{ Locus } \\
\hline & & & & Aat-1 & Aat-2 & Idh-1 & Ldh-1 & Ldh-2 & Mdh-1 & Mpi & Pgi & Pgm-1 & Pgm-2 & Sod-1 & blood \\
\hline 69 & 158 & $\mathrm{~F}$ & 165946 & $\mathrm{BD}$ & $\mathrm{AB}$ & A & $\mathrm{BC}$ & B & $\mathrm{BD}$ & $\mathrm{BC}$ & - & $\mathrm{BC}$ & B & BD & 561 \\
\hline 70) & 159 & $\mathrm{~F}$ & 160225 & BD & $\mathrm{AB}$ & $\mathrm{AB}$ & $\mathrm{BC}$ & B & BD & $\mathrm{BC}$ & $\mathrm{AB}$ & $\mathrm{BC}$ & B & D & 740 \\
\hline 71 & 169 & $\mathrm{~F}$ & 167006 & BD & $\mathrm{AB}$ & $\mathrm{AB}$ & $\mathrm{BC}$ & B & $\mathrm{BD}$ & $\mathrm{BC}$ & - & $\mathrm{BC}$ & B & $\mathrm{BD}$ & 752 \\
\hline 72 & 174 & $\mathrm{~F}$ & 169928 & BD & $\mathrm{AB}$ & $\mathrm{AB}$ & $\mathrm{BC}$ & B & BD & - & AC & B & B & BD & FCM \\
\hline 73 & 192 & $\mathrm{~F}$ & 169767 & BD & $\mathrm{AB}$ & $\mathrm{AB}$ & $\mathrm{BC}$ & B & BD & $\mathrm{AB}$ & - & B & B & BD & FCM \\
\hline 74 & 201 & $\mathrm{~F}$ & 165947 & BD & $\mathrm{AB}$ & $\mathrm{AB}$ & $\mathrm{BC}$ & B & BD & - & - & B & B & BD & 660 \\
\hline 75 & 201 & $\mathrm{~F}$ & 165948 & BD & $\mathrm{AB}$ & $\mathrm{AB}$ & $\mathrm{BC}$ & $\mathrm{C}$ & BD & $\mathrm{BC}$ & - & B & B & BD & 700 \\
\hline 76 & 201 & $\mathrm{~F}$ & 165949 & BD & $\mathrm{AB}$ & $\mathrm{AB}$ & $\mathrm{BC}$ & $\mathrm{C}$ & BD & - & - & B & B & BD & 789 \\
\hline 77 & 201 & $\mathrm{~F}$ & 165950 & BD & $\mathrm{AB}$ & $\mathrm{AB}$ & $\mathrm{BC}$ & C & BD & $\mathrm{BC}$ & - & B & B & BD & 762 \\
\hline 78 & 201 & $\mathrm{~F}$ & 165951 & BD & $\mathrm{AB}$ & $\mathrm{AB}$ & $\mathrm{BC}$ & $\mathrm{C}$ & BD & - & - & B & B & BD & 667 \\
\hline 79 & 201 & $\mathrm{~F}$ & 165952 & BD & $\mathrm{AB}$ & $\mathrm{AB}$ & $\mathrm{BC}$ & $\mathrm{C}$ & BD & $\mathrm{BC}$ & - & B & B & BD & 574 \\
\hline 80) & 201 & $\mathrm{~F}$ & 169766 & $\mathrm{BD}$ & $\mathrm{AB}$ & $\mathrm{AB}$ & $\mathrm{BC}$ & $\mathrm{BC}$ & $\mathrm{BD}$ & $\mathrm{BC}$ & - & B & B & BD & $\mathrm{FCM}$ \\
\hline 81 & 211 & $\mathrm{~F}$ & 160246 & BD & $\mathrm{AB}$ & $\mathrm{AB}$ & $\mathrm{BC}$ & B & BD & $\mathrm{BC}$ & - & B & B & BD & 742 \\
\hline 82 & 211 & $\mathrm{~F}$ & 160247 & BD & $\mathrm{AB}$ & $\mathrm{AB}$ & $\mathrm{BC}$ & B & $\mathrm{BD}$ & $\mathrm{BC}$ & - & B & B & $\mathrm{BD}$ & 696 \\
\hline 83 & 214 & $\mathrm{~F}$ & 158785 & BD & $\mathrm{AB}$ & $\mathrm{AB}$ & $\mathrm{BC}$ & B & BD & $\mathrm{BC}$ & $\mathrm{AC}$ & $\mathrm{BC}$ & B & BD & 729 \\
\hline 84 & 214 & $\mathrm{~F}$ & 158786 & $\mathrm{BD}$ & $\mathrm{AB}$ & $\mathrm{AB}$ & $\mathrm{BC}$ & B & $\mathrm{BD}$ & $\mathrm{BC}$ & - & $\mathrm{BC}$ & B & $\mathrm{BD}$ & 732 \\
\hline 85 & 215 & $\mathrm{~F}$ & 158812 & $\mathrm{BD}$ & $\mathrm{AB}$ & $\mathrm{AB}$ & $\mathrm{BC}$ & B & BD & $\mathrm{AB}$ & $\mathrm{AC}$ & B & B & BD & 611 \\
\hline 86 & 215 & $\mathrm{~F}$ & 158816 & $\mathrm{BD}$ & $\mathrm{AB}$ & $\mathrm{AB}$ & $\mathrm{BC}$ & B & BD & $\mathrm{AB}$ & $\mathrm{AC}$ & B & B & $\mathrm{BD}$ & 638 \\
\hline
\end{tabular}

Appendix 2-4: Genotypes of triploid (3n) Ambystoma 2 laterale - jeffersonianum (LLJ) specimens ordered by site number.

Locus

Site Sex AMNH Aat-1 Aat-2 Idh-1 Ldh-1 Ldh-2 Mdh-1 Mpi Pgi Pgm-1 Pgm-2 Sod-1 blood

\begin{tabular}{|c|c|c|c|c|c|c|c|c|c|c|c|c|c|c|c|}
\hline 1 & 7 & $\mathrm{~F}$ & 164593 & BBD & $\mathrm{ABB}$ & $\mathrm{ABB}$ & $\mathrm{BBC}$ & $\mathrm{ABB}$ & BDD & $\mathrm{BCC}$ & - & B & B & BBD & 983 \\
\hline 2 & 18 & $\mathrm{~F}$ & 165994 & BBD & $\mathrm{ABB}$ & $\mathrm{ABB}$ & $\mathrm{BBC}$ & B & BDD & - & - & & & 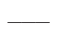 & 951 \\
\hline & 42 & $\mathrm{~F}$ & 166983 & BBD & AAB & $\mathrm{ABB}$ & $\mathrm{BBC}$ & B & BBD & BCC & & $\mathrm{BBC}$ & B & BBD & 990 \\
\hline & 42 & $\mathrm{~F}$ & 166984 & BBD & $\mathrm{ABB}$ & $\mathrm{ABB}$ & $\mathrm{BBC}$ & B & BBD & BCC & - & B & B & BBD & 964 \\
\hline 5 & 42 & $\mathrm{~F}$ & 166985 & BBD & $\mathrm{ABB}$ & $\mathrm{ABB}$ & $\mathrm{BBC}$ & B & BBD & BCC & - & B & E & BBD & 1093 \\
\hline & 42 & $\mathrm{~F}$ & 166986 & BBD & $\mathrm{ABB}$ & $\mathrm{ABB}$ & $\mathrm{BBC}$ & $\mathrm{AB}$ & BDD & BCC & $\mathrm{BCC}$ & B & & BD & 1006 \\
\hline & 42 & $\mathrm{~F}$ & 166987 & BBD & $\mathrm{ABB}$ & $\mathrm{ABB}$ & $\mathrm{BBC}$ & B & BBD & BCC & . & $\mathrm{BBC}$ & & BBD & 902 \\
\hline 8 & 107 & $\mathrm{~F}$ & 169900 & BBD & $\mathrm{ABB}$ & $\mathrm{ABB}$ & $\mathrm{BBC}$ & A & BDD & - & $\mathrm{BCC}$ & B & & BBD & FCM \\
\hline & 107 & $\mathrm{~F}$ & 169901 & BBD & $\mathrm{ABB}$ & B & $\mathrm{BBC}$ & A & BDD & 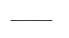 & BCC & $\mathrm{BBC}$ & E & BBD & FC \\
\hline 10) & 10 & $\mathrm{~F}$ & 169 & BBD & $\mathrm{ABB}$ & $\mathrm{ABB}$ & $\mathrm{BBC}$ & $\mathrm{P}$ & BDD & - & BCC & B & & BD & $\mathrm{FCl}$ \\
\hline 1 & 107 & $\mathrm{~F}$ & 169903 & BBD & $\mathrm{ABB}$ & B & $\mathrm{BBC}$ & B & BDD & - & $\mathrm{BCC}$ & B & & BBD & $\mathrm{FCM}$ \\
\hline 124 & 107 & $\mathrm{~F}$ & 169904 & BBD & $\mathrm{ABB}$ & $\mathrm{ABB}$ & $\mathrm{BBC}$ & B & BDD & - & $\mathrm{BCC}$ & B & $\mathrm{E}$ & BBD & FCM \\
\hline 13 & 107 & $\mathrm{~F}$ & 169905 & BBD & $\mathrm{ABB}$ & $\mathrm{ABB}$ & $\mathrm{BBC}$ & A & BDD & - & $\mathrm{BCC}$ & B & $\mathrm{E}$ & BD & $\mathrm{FCl}$ \\
\hline 4 & 10 & $\mathrm{~F}$ & 169 & BBD & $\mathrm{ABB}$ & $\mathrm{ABB}$ & $\mathrm{BBC}$ & $\mathrm{B}$ & BDD & - & $\mathrm{ABC}$ & B & $\mathrm{E}$ & BD & FCM \\
\hline & 108 & M & 160 & BBD & & B & $\mathrm{BBC}$ & B & BDD & - & - & B & & BBD & 796 \\
\hline 16 & 108 & $\mathrm{~F}$ & 160269 & BBD & $\mathrm{ABB}$ & $\mathrm{ABB}$ & $\mathrm{BBC}$ & B & BDD & BCC & $\mathrm{BCC}$ & $\mathrm{BBC}$ & B & BBD & 1058 \\
\hline 17 & 108 & $\mathrm{~F}$ & 160270 & BBD & $\mathrm{ABB}$ & $\mathrm{ABB}$ & B & B & BDD & BCC & - & $\mathrm{BBC}$ & B & BBD & 996 \\
\hline 18 & 108 & $\mathrm{~F}$ & 160271 & BBD & $\mathrm{ABB}$ & B & $\mathrm{BBC}$ & B & BDD & BCC & - & B & B & BBD & 1178 \\
\hline 9 & 108 & $\mathrm{~F}$ & 16 & BBD & $\mathrm{ABB}$ & $\mathrm{ABB}$ & $\mathrm{BBC}$ & B & BDD & BCC & - & $\mathrm{BB}$ & $\mathrm{E}$ & BD & 1195 \\
\hline 20) & 10 & $\mathrm{~F}$ & & BBD & $\mathrm{ABB}$ & $\mathrm{ABB}$ & $\mathrm{BBC}$ & B & BDD & BCC & - & B & I & BDD & 1170 \\
\hline 21 & 108 & $\mathrm{~F}$ & 160274 & BBD & $\mathrm{ABB}$ & $\mathrm{ABB}$ & $\mathrm{BBC}$ & A & BDD & BCC & AAC & B & D & BBD & 1194 \\
\hline 22 & 108 & $\mathrm{~F}$ & 160275 & BBD & $\mathrm{ABB}$ & $\mathrm{ABB}$ & $\mathrm{BBC}$ & B & BDD & BCC & AAC & $\mathrm{BCC}$ & B & BBD & 1061 \\
\hline
\end{tabular}


APPENDIX 2-4

(Continued)

Locus

Site Sex AMNH Aat-1 Aat-2 Idh-1 Ldh-1 Ldh-2 Mdh-1 Mpi Pgi Pgm-1 Pgm-2 Sod-1 blood

\begin{tabular}{|c|c|c|c|c|c|c|c|c|c|c|c|c|c|c|c|}
\hline 23 & 108 & $\mathrm{~F}$ & 160276 & $\mathrm{BBD}$ & $\mathrm{ABB}$ & B & $\mathrm{BBC}$ & B & BDD & $\mathrm{BCC}$ & $\mathrm{ACC}$ & $\mathrm{BBC}$ & B & $\mathrm{BBD}$ & 792 \\
\hline 24 & 108 & $\mathrm{~F}$ & 160277 & BBD & $\mathrm{ABB}$ & $\mathrm{ABB}$ & $\mathrm{BBC}$ & B & BDD & - & AAC & B & B & BBD & 1137 \\
\hline 25 & 108 & F & 160278 & BBD & $\mathrm{ABB}$ & $\mathrm{ABB}$ & $\mathrm{BBC}$ & A & BDD & $\mathrm{BCC}$ & AAC & $\mathrm{BBC}$ & B & BBD & 1029 \\
\hline 26 & 108 & $\mathrm{~F}$ & 160279 & BBD & $\mathrm{ABB}$ & $\mathrm{ABB}$ & $\mathrm{BBC}$ & B & BDD & $\mathrm{BCC}$ & AAC & $\mathrm{BCC}$ & B & BBD & 1062 \\
\hline 27 & 108 & $\mathrm{~F}$ & 160280 & BDD & $\mathrm{ABB}$ & $\mathrm{ABB}$ & $\mathrm{BBC}$ & B & BDD & $\mathrm{BCC}$ & AAC & $\mathrm{BBC}$ & B & BBD & 950 \\
\hline 28 & 108 & $\mathrm{~F}$ & 160281 & BDD & $\mathrm{ABB}$ & $\mathrm{ABB}$ & $\mathrm{BBC}$ & A & BDD & $\mathrm{BCC}$ & $\mathrm{AAC}$ & $\mathrm{BCC}$ & B & $\mathrm{BBD}$ & 893 \\
\hline 29 & 108 & $\mathrm{~F}$ & 160282 & BDD & $\mathrm{ABB}$ & $\mathrm{ABB}$ & $\mathrm{BBC}$ & A & BBD & $\mathrm{BCC}$ & $\mathrm{AAC}$ & $\mathrm{BBC}$ & B & BBD & 913 \\
\hline 30) & 108 & $\mathrm{~F}$ & 160283 & BBD & $\mathrm{ABB}$ & $\mathrm{ABB}$ & $\mathrm{BBC}$ & B & BDD & $\mathrm{BCC}$ & $\mathrm{ACC}$ & $\mathrm{BCC}$ & B & BBD & 1186 \\
\hline 31 & 108 & $\mathrm{~F}$ & 160284 & BBD & $\mathrm{ABB}$ & $\mathrm{ABB}$ & $\mathrm{BBC}$ & A & BDD & - & $\mathrm{AAC}$ & B & B & BBD & 1024 \\
\hline 32 & 110 & $\mathrm{~F}$ & 165995 & BBD & $\mathrm{ABB}$ & $\mathrm{ABB}$ & $\mathrm{BBC}$ & A & BDD & $\mathrm{BCC}$ & - & B & B & BBD & 959 \\
\hline 33 & 110 & $\mathrm{~F}$ & 169396 & BBD & $\mathrm{ABB}$ & - & - & 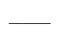 & BDD & A & - & B & B & BBD & FCM \\
\hline 34 & 110 & F & 169397 & BBD & $\mathrm{ABB}$ & $\mathrm{ABB}$ & $\mathrm{BBC}$ & B & BDD & $\mathrm{BCC}$ & $\mathrm{C}$ & B & B & BBD & FCM \\
\hline 35 & 110 & $\mathrm{~F}$ & 169398 & BBD & $\mathrm{ABB}$ & $\mathrm{ABB}$ & $\mathrm{BBC}$ & B & BDD & $\mathrm{BCC}$ & $\mathrm{ACC}$ & B & B & BBD & FCM \\
\hline 36 & 110 & $\mathrm{~F}$ & 169894 & $\mathrm{BBD}$ & $\mathrm{ABB}$ & $\mathrm{ABB}$ & $\mathrm{BBC}$ & B & BDD & $\mathrm{BCC}$ & $\mathrm{BCC}$ & $\mathrm{BBC}$ & B & $\mathrm{BBD}$ & FCM \\
\hline 37 & 110 & $\mathrm{~F}$ & 169895 & BBD & $\mathrm{ABB}$ & $\mathrm{AAB}$ & $\mathrm{BBC}$ & B & BBD & $\mathrm{BCC}$ & $\mathrm{ACC}$ & $\mathrm{BBC}$ & B & BBD & FCM \\
\hline 38 & 110 & $\mathrm{~F}$ & 169896 & BBD & $\mathrm{ABB}$ & $\mathrm{ABB}$ & $\mathrm{BBC}$ & B & BDD & BCC & $\mathrm{BCC}$ & $\mathrm{BBC}$ & B & BBD & FCM \\
\hline 39 & 110 & $\mathrm{~F}$ & 169897 & BBD & $\mathrm{ABB}$ & $\mathrm{ABB}$ & $\mathrm{BBC}$ & B & BDD & $\mathrm{BCC}$ & $\mathrm{ACC}$ & B & B & BBD & FCM \\
\hline 40) & 110 & $\mathrm{~F}$ & 169898 & $\mathrm{BBD}$ & $\mathrm{ABB}$ & $\mathrm{ABB}$ & $\mathrm{BBC}$ & B & $\mathrm{BDD}$ & $\mathrm{BCC}$ & $\mathrm{BCC}$ & B & B & BBD & FCM \\
\hline 41 & 111 & $\mathrm{~F}$ & 169385 & BBD & $\mathrm{ABB}$ & $\mathrm{ABB}$ & $\mathrm{BBC}$ & B & BDD & $\mathrm{C}$ & $\mathrm{BCC}$ & B & B & B & FCM \\
\hline 42 & 111 & $\mathrm{~F}$ & 169399 & BBD & $\mathrm{ABB}$ & $\mathrm{ABB}$ & $\mathrm{BBC}$ & $\mathrm{BBC}$ & BBD & $\mathrm{BCC}$ & $\mathrm{ACC}$ & B & B & BBD & FCM \\
\hline 43 & 111 & $\mathrm{~F}$ & 169400 & BBD & $\mathrm{ABB}$ & $\mathrm{ABB}$ & $\mathrm{BBC}$ & B & BDD & $\mathrm{BCC}$ & $\mathrm{ABC}$ & B & B & BBD & FCM \\
\hline 44 & 111 & $\mathrm{~F}$ & 169401 & BBD & $\mathrm{ABB}$ & $\mathrm{ABB}$ & $\mathrm{BBC}$ & B & BDD & $\mathrm{BCC}$ & $\mathrm{ACC}$ & B & B & BBD & FCM \\
\hline 45 & 111 & $\mathrm{~F}$ & 169402 & BBD & $\mathrm{ABB}$ & $\mathrm{ABB}$ & $\mathrm{BBC}$ & B & BDD & $\mathrm{BCC}$ & $\mathrm{ACC}$ & B & B & BBD & FCM \\
\hline 46 & 111 & $\mathrm{~F}$ & 169403 & BBD & $\mathrm{ABB}$ & - & $\mathrm{BBC}$ & B & BDD & $\mathrm{ABB}$ & - & B & B & BBD & FCM \\
\hline 47 & 111 & $\mathrm{~F}$ & 169404 & BBD & $\mathrm{ABB}$ & - & - & 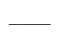 & BDD & $\mathrm{BCC}$ & - & B & B & BBD & FCM \\
\hline 48 & 111 & $\mathrm{~F}$ & 169405 & $\mathrm{BBD}$ & $\mathrm{ABB}$ & - & - & 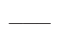 & $\mathrm{BDD}$ & $\mathrm{BCC}$ & - & B & B & BBD & FCM \\
\hline 49 & 111 & $\mathrm{~F}$ & 169406 & $\mathrm{BBD}$ & $\mathrm{ABB}$ & - & $\mathrm{BBC}$ & B & $\mathrm{BDD}$ & $\mathrm{BCC}$ & - & B & B & BBD & FCM \\
\hline 50) & 111 & $\mathrm{~F}$ & 169407 & BBD & $\mathrm{ABB}$ & - & $\mathrm{BBC}$ & B & BDD & $\mathrm{BCC}$ & - & B & B & BBD & FCM \\
\hline 51 & 111 & $\mathrm{~F}$ & 169408 & BBD & $\mathrm{ABB}$ & $\mathrm{ABB}$ & $\mathrm{BBC}$ & B & BDD & B & $\mathrm{ACC}$ & B & B & B & FCM \\
\hline 52 & 111 & $\mathrm{~F}$ & 169409 & $\mathrm{BBD}$ & $\mathrm{ABB}$ & $\mathrm{ABB}$ & $\mathrm{BBC}$ & B & BDD & $\mathrm{BCC}$ & $\mathrm{AAC}$ & B & B & BBD & FCM \\
\hline 53 & 111 & $\mathrm{~F}$ & 169410 & $\mathrm{BBD}$ & $\mathrm{ABB}$ & $\mathrm{ABB}$ & $\mathrm{BBC}$ & B & BDD & $\mathrm{BCC}$ & $\mathrm{ACC}$ & B & B & BBD & FCM \\
\hline 54 & 111 & $\mathrm{~F}$ & 169806 & BBD & $\mathrm{ABB}$ & $\mathrm{ABB}$ & $\mathrm{BBC}$ & B & BDD & $\mathrm{BCC}$ & - & B & B & BBD & FCM \\
\hline 55 & 112 & $\mathrm{~F}$ & 169803 & BBD & $\mathrm{ABB}$ & B & $\mathrm{BBC}$ & B & $\mathrm{BD}$ & $\mathrm{BCC}$ & $\mathrm{ACC}$ & B & B & BBD & FCM \\
\hline 56 & 112 & $\mathrm{~F}$ & 169804 & BBD & $\mathrm{ABB}$ & B & $\mathrm{BBC}$ & B & BDD & $\mathrm{BCC}$ & - & B & B & BBD & FCM \\
\hline 57 & 112 & $\mathrm{~F}$ & 169805 & BBD & $\mathrm{ABB}$ & $\mathrm{ABB}$ & $\mathrm{BBC}$ & B & BDD & $\mathrm{BCC}$ & - & $\mathrm{BBC}$ & B & BBD & FCM \\
\hline 58 & 112 & M & 169807 & BBD & $\mathrm{ABB}$ & B & B & B & BDD & $\mathrm{BCC}$ & - & $\mathrm{BBC}$ & B & BBD & - \\
\hline 59 & 113 & $\mathrm{~F}$ & 162942 & BBD & $\mathrm{ABB}$ & $\mathrm{ABB}$ & $\mathrm{BBC}$ & B & BDD & $\mathrm{BCC}$ & $\mathrm{BCC}$ & $\mathrm{BBC}$ & B & BBD & 994 \\
\hline 60) & 113 & $\mathrm{~F}$ & 162943 & BBD & $\mathrm{ABB}$ & $\mathrm{ABB}$ & $\mathrm{BBC}$ & B & BDD & $\mathrm{BCC}$ & $\mathrm{C}$ & $\mathrm{BBC}$ & B & BBD & 1043 \\
\hline 61 & 113 & $\mathrm{~F}$ & 162944 & $\mathrm{BBD}$ & $\mathrm{ABB}$ & $\mathrm{ABB}$ & $\mathrm{BBC}$ & B & BDD & $\mathrm{BCC}$ & $\mathrm{AAC}$ & B & B & BBD & 994 \\
\hline 62 & 113 & $\mathrm{~F}$ & 162945 & BBD & $\mathrm{ABB}$ & $\mathrm{ABB}$ & $\mathrm{BBC}$ & B & BDD & $\mathrm{BCC}$ & $\mathrm{AAC}$ & B & B & BBD & 1027 \\
\hline 63 & 113 & $\mathrm{~F}$ & 162946 & BBD & $\mathrm{ABB}$ & $\mathrm{ABB}$ & $\mathrm{BBC}$ & B & BDD & $\mathrm{BCC}$ & $\mathrm{C}$ & $\mathrm{BCC}$ & B & BBD & 806 \\
\hline 64 & 113 & $\mathrm{~F}$ & 162947 & BBD & $\mathrm{ABB}$ & $\mathrm{AAB}$ & $\mathrm{BBC}$ & B & BDD & $\mathrm{BCC}$ & - & B & B & BBD & 1196 \\
\hline 65 & 113 & $\mathrm{~F}$ & 162948 & BBD & $\mathrm{ABB}$ & $\mathrm{ABB}$ & $\mathrm{BBC}$ & B & BDD & $\mathrm{BCC}$ & - & BBD & B & BBD & 1154 \\
\hline 66 & 113 & $\mathrm{~F}$ & 162949 & BBD & $\mathrm{ABB}$ & $\mathrm{ABB}$ & $\mathrm{AAC}$ & B & BDD & $\mathrm{BCC}$ & $\mathrm{ACC}$ & B & B & $\mathrm{BBD}$ & 923 \\
\hline 67 & 113 & $\mathrm{~F}$ & 162951 & BBD & $\mathrm{ABB}$ & A & $\mathrm{BBC}$ & B & BBD & $\mathrm{BCC}$ & $\mathrm{ACC}$ & B & B & BBD & 1005 \\
\hline 68 & 113 & $\mathrm{~F}$ & 162952 & BBD & $\mathrm{ABB}$ & $\mathrm{AAB}$ & $\mathrm{BBC}$ & B & BDD & $\mathrm{BBC}$ & $\mathrm{AAC}$ & B & B & BBD & 982 \\
\hline 69 & 113 & $\mathrm{~F}$ & 162953 & BBD & $\mathrm{ABB}$ & $\mathrm{ABB}$ & $\mathrm{BBC}$ & B & BDD & $\mathrm{BCC}$ & $\mathrm{ABC}$ & B & B & BBD & 1038 \\
\hline 70) & 113 & $\mathrm{~F}$ & 162954 & BBD & $\mathrm{ABB}$ & $\mathrm{ABB}$ & $\mathrm{BBC}$ & B & BDD & $\mathrm{BCC}$ & $\mathrm{ACC}$ & B & B & BBD & 1110 \\
\hline 71 & 113 & $\mathrm{~F}$ & 162955 & BDD & $\mathrm{ABB}$ & A & $\mathrm{BBC}$ & B & BBD & BCC & $\mathrm{ACC}$ & B & B & BBD & 833 \\
\hline 72 & 113 & $\mathrm{~F}$ & 162956 & BBD & $\mathrm{ABB}$ & A & $\mathrm{BBC}$ & B & BBD & - & $\mathrm{BCC}$ & B & B & BBD & 906 \\
\hline 73 & 113 & $\mathrm{~F}$ & 162957 & BBD & $\mathrm{ABB}$ & $\mathrm{ABB}$ & $\mathrm{BBC}$ & B & BDD & $\mathrm{BCC}$ & AAC & B & B & BBD & 996 \\
\hline 74 & 113 & $\mathrm{~F}$ & 162958 & BBD & $\mathrm{ABB}$ & $\mathrm{ABB}$ & $\mathrm{BBC}$ & B & BDD & $\mathrm{BCC}$ & $\mathrm{BCC}$ & B & B & BBD & 962 \\
\hline
\end{tabular}


APPENDIX 2-4

(Continued)

\begin{tabular}{|c|c|c|c|c|c|c|c|c|c|c|c|c|c|c|c|}
\hline & \multirow[b]{2}{*}{ Site } & \multirow[b]{2}{*}{ Sex } & \multirow[b]{2}{*}{ AMNH } & \multicolumn{12}{|c|}{ Locus } \\
\hline & & & & Aat-1 & Aat-2 & Idh-1 & Ldh-1 & Ldh-2 & Mdh-1 & Mpi & Pgi & Pgm-1 & Pgm-2 & Sod-1 & blood \\
\hline 75 & 113 & $\mathrm{~F}$ & 162959 & BBD & $\mathrm{ABB}$ & $\mathrm{ABB}$ & $\mathrm{BBC}$ & B & BDD & $\mathrm{BCC}$ & $\mathrm{BCC}$ & B & B & BBD & 925 \\
\hline 76 & 123 & $\mathrm{~F}$ & 165996 & BBD & $\mathrm{ABB}$ & $\mathrm{ABB}$ & $\mathrm{BBC}$ & B & BBD & . & & 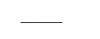 & & $\overline{.}$ & 670 \\
\hline 77 & 125 & $\mathrm{~F}$ & 169815 & BBD & $\mathrm{ABB}$ & $\mathrm{ABB}$ & $\mathrm{BBC}$ & $\mathrm{ABB}$ & $\mathrm{D}$ & $\mathrm{BCC}$ & $\mathrm{BCC}$ & $\mathrm{BBC}$ & B & BBD & FCM \\
\hline 78 & 127 & $\mathrm{~F}$ & 160257 & BBD & ABB & $\mathrm{ABB}$ & BCC & $\mathrm{BBC}$ & BDD & BCC & BCC & B & B & BBD & 1056 \\
\hline 79) & 127 & $\mathrm{~F}$ & 160258 & BBD & $\mathrm{ABB}$ & $\mathrm{ABB}$ & $\mathrm{BCC}$ & $\mathrm{BBC}$ & BDD & C & AAC & $\mathrm{BBC}$ & B & BBD & 929 \\
\hline 80 & 127 & $\mathrm{~F}$ & 160259 & BBD & $\mathrm{ABB}$ & $\mathrm{ABB}$ & $\mathrm{BBC}$ & B & BDD & $\mathrm{C}$ & BCC & BBC & B & BBD & 1199 \\
\hline 82 & 127 & $\mathrm{~F}$ & 160260 & BBD & $\mathrm{ABB}$ & $\mathrm{ABB}$ & B & B & BDD & - & - & BBC & B & BBD & 1007 \\
\hline 83 & 127 & $\mathrm{~F}$ & 160261 & BBD & $\mathrm{ABB}$ & $\mathrm{ABB}$ & $\mathrm{BBC}$ & B & BDD & - & - & B & B & BBD & 1252 \\
\hline 84 & 127 & $\mathrm{~F}$ & 160262 & BBD & $\mathrm{ABB}$ & $\mathrm{ABB}$ & - & B & BDD & 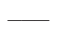 & - & BBC & B & BBD & 1032 \\
\hline 81 & 127 & $\mathrm{~F}$ & 160263 & BBD & $\mathrm{ABB}$ & $\mathrm{ABB}$ & $\mathrm{BBC}$ & B & BDD & $\mathrm{C}$ & $\mathrm{C}$ & BBC & B & B & 1121 \\
\hline 85 & 127 & $\mathrm{~F}$ & 160264 & BBD & $\mathrm{ABB}$ & $\mathrm{ABB}$ & $\mathrm{BBC}$ & B & BDD & C & BCC & BBC & B & BBD & 1048 \\
\hline 86 & 127 & $\mathrm{~F}$ & 160265 & BBD & $\mathrm{ABB}$ & $\mathrm{ABB}$ & $\mathrm{BBC}$ & B & BDD & - & - & B & B & BBD & 926 \\
\hline 87 & 127 & $\mathrm{~F}$ & 160266 & BBD & $\mathrm{ABB}$ & $\mathrm{ABB}$ & - & - & BDD & - & - & $\mathrm{BBC}$ & B & BBD & 988 \\
\hline 88 & 128 & $\mathrm{~F}$ & 160268 & BBD & $\mathrm{ABB}$ & $\mathrm{ABB}$ & $\mathrm{BBC}$ & A & BDD & - & - & B & B & BBD & 911 \\
\hline 89 & 129 & $\mathrm{~F}$ & 162961 & BBD & $\mathrm{ABB}$ & $\mathrm{ABB}$ & $\mathrm{BBC}$ & B & BDD & - & C & B & B & BBD & 1235 \\
\hline 90) & 129 & $\mathrm{~F}$ & 165997 & BBD & $\mathrm{ABB}$ & $\mathrm{ABB}$ & BCC & B & BDD & C & C & $\mathrm{BBC}$ & B & BBD & 996 \\
\hline 91 & 129 & $\mathrm{~F}$ & 165998 & BBD & $\mathrm{ABB}$ & $\mathrm{ABB}$ & $\mathrm{BBC}$ & B & BDD & $\mathrm{C}$ & $\mathrm{C}$ & B & B & BBD & 916 \\
\hline 92 & 129 & $\mathrm{~F}$ & 165999 & BBD & $\mathrm{ABB}$ & $\mathrm{ABB}$ & $\mathrm{BBC}$ & B & BDD & C & C & BBC & B & BBD & 1063 \\
\hline 93 & 132 & $\mathrm{~F}$ & 162960 & BBD & ABB & $\mathrm{ABB}$ & $\mathrm{BBC}$ & B & BDD & $\mathrm{C}$ & AAC & $\mathrm{BBC}$ & B & BBD & 909 \\
\hline 94 & 133 & $\mathrm{~F}$ & 166000 & BBD & $\mathrm{ABB}$ & $\mathrm{ABB}$ & BCC & B & BDD & BCC & C & BBC & B & BBD & 841 \\
\hline 95 & 133 & $\mathrm{~F}$ & 166001 & BBD & $\mathrm{ABB}$ & $\mathrm{ABB}$ & $\mathrm{BBC}$ & B & BDD & $\mathrm{BCC}$ & 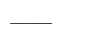 & BBC & B & BBD & 828 \\
\hline 96 & 133 & $\mathrm{~F}$ & 166002 & BBD & $\mathrm{ABB}$ & $\mathrm{ABB}$ & $\mathrm{BBC}$ & B & BDD & BCC & - & BBC & B & BBD & 1088 \\
\hline 97 & 133 & $\mathrm{~F}$ & 166003 & BBD & $\mathrm{ABB}$ & $\mathrm{ABB}$ & $\mathrm{BBC}$ & B & BDD & BCC & $\mathrm{C}$ & BBC & B & BBD & 1184 \\
\hline 98 & 136 & $\mathrm{~F}$ & 166004 & BBD & $\mathrm{ABB}$ & $\mathrm{ABB}$ & $\mathrm{BBC}$ & B & BDD & BCC & C & BBC & B & BBD & 944 \\
\hline 99 & 136 & $\mathrm{~F}$ & 166005 & BBD & $\mathrm{ABB}$ & $\mathrm{ABB}$ & $\mathrm{BBC}$ & B & BDD & $\mathrm{BCC}$ & - & B & B & BBD & 966 \\
\hline 100) & 136 & $\mathrm{~F}$ & 166006 & BBD & $\mathrm{ABB}$ & $\mathrm{ABB}$ & $\mathrm{BBC}$ & B & BDD & BCC & - & B & B & BBD & 987 \\
\hline 101 & 136 & $\mathrm{~F}$ & 166007 & BBD & $\mathrm{ABB}$ & B & $\mathrm{BBC}$ & B & BDD & BCC & - & $\mathrm{BBC}$ & B & BBD & 1071 \\
\hline 102 & 136 & $\mathrm{~F}$ & 166008 & BBD & $\mathrm{ABB}$ & $\mathrm{ABB}$ & $\mathrm{BBC}$ & B & BDD & BCC & & BBC & B & BBD & 1031 \\
\hline 103 & 136 & $\mathrm{~F}$ & 166009 & BBD & $\mathrm{ABB}$ & $\mathrm{ABB}$ & $\mathrm{BBC}$ & B & BDD & - & C & B & B & BBD & 922 \\
\hline 104 & 136 & $\mathrm{~F}$ & 166010 & BBD & $\mathrm{ABB}$ & $\mathrm{ABB}$ & $\mathrm{BBC}$ & B & BDD & - & C & BBC & B & BBD & 1001 \\
\hline 105 & 136 & $\mathrm{~F}$ & 166011 & BBD & $\mathrm{ABB}$ & $\mathrm{ABB}$ & $\mathrm{BBC}$ & B & BDD & - & C & BBC & B & BBD & 1000 \\
\hline 106 & 136 & $\mathrm{~F}$ & 166012 & BBD & $\mathrm{ABB}$ & $\mathrm{ABB}$ & $\mathrm{BBC}$ & B & BDD & - & ACC & BBC & B & BBD & 1200 \\
\hline 107 & 136 & $\mathrm{~F}$ & 166013 & BBD & $\mathrm{ABB}$ & $\mathrm{ABB}$ & $\mathrm{BBC}$ & B & BDD & BCC & - & BBC & B & BBD & 879 \\
\hline 108 & 136 & $\mathrm{~F}$ & 166014 & BBD & $\mathrm{ABB}$ & $\mathrm{ABB}$ & $\mathrm{BBC}$ & B & BDD & BCC & - & BBC & B & BBD & 1033 \\
\hline 109 & 143 & $\mathrm{~F}$ & 167033 & BBD & $\mathrm{ABB}$ & - & - & - & - & BCC & - & B & B & BBD & 1194 \\
\hline 110) & 143 & $\mathrm{~F}$ & 167034 & BBD & $\mathrm{ABB}$ & $\mathrm{ABB}$ & $\mathrm{BBC}$ & B & BDD & $\mathrm{BCC}$ & $\mathrm{BCC}$ & B & B & BBD & 1022 \\
\hline 111 & 143 & $\mathrm{~F}$ & 167035 & BBD & $\mathrm{ABB}$ & $\mathrm{ABB}$ & $\mathrm{BBC}$ & B & BDD & BCC & $\mathrm{ABC}$ & B & B & BBD & 1018 \\
\hline 112 & 143 & $\mathrm{~F}$ & 167036 & BBD & $\mathrm{ABB}$ & - & $\mathrm{BBC}$ & B & BDD & BCC & BCC & B & B & BBD & 1065 \\
\hline 113 & 144 & $\mathrm{~F}$ & 166015 & BBD & $\mathrm{ABB}$ & $\mathrm{AAB}$ & $\mathrm{BBC}$ & B & BDD & - & C & B & B & BBD & 689 \\
\hline 114 & 144 & $\mathrm{~F}$ & 166016 & BBD & $\mathrm{ABB}$ & $\mathrm{ABB}$ & $\mathrm{BBC}$ & B & BDD & BCC & 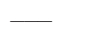 & $\mathrm{BBC}$ & B & BBD & 951 \\
\hline 115 & 144 & $\mathrm{~F}$ & 166017 & BBD & $\mathrm{ABB}$ & $\mathrm{ABB}$ & $\mathrm{BBC}$ & B & BDD & BCC & - & BBC & B & BBD & 971 \\
\hline 116 & 145 & $\mathrm{~F}$ & 167041 & BBD & $\mathrm{ABB}$ & $\mathrm{ABB}$ & $\mathrm{BBC}$ & B & BDD & BCC & $\mathrm{BCC}$ & B & B & BBD & 990 \\
\hline 117 & 145 & $\mathrm{~F}$ & 167042 & BBD & $\mathrm{ABB}$ & $\mathrm{ABB}$ & BCC & B & BDD & BCC & BCC & B & B & BBD & 997 \\
\hline 118 & 145 & $\mathrm{~F}$ & 167043 & BBD & $\mathrm{ABB}$ & $\mathrm{ABB}$ & $\mathrm{BBC}$ & B & BDD & BCC & $\mathrm{ABC}$ & 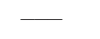 & 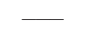 & BBD & 1046 \\
\hline 119 & 145 & $\mathrm{~F}$ & 167044 & BBD & $\mathrm{ABB}$ & $\mathrm{ABB}$ & $\mathrm{BBC}$ & B & BDD & BCC & $\mathrm{ABC}$ & B & B & BBD & 1069 \\
\hline 120) & 145 & $\mathrm{~F}$ & 167045 & BBD & $\mathrm{ABB}$ & $\mathrm{ABB}$ & $\mathrm{BCC}$ & B & BDD & BCC & $\mathrm{ABC}$ & & & BBD & 1038 \\
\hline 121 & 145 & $\mathrm{~F}$ & 167046 & BBD & ABB & $\mathrm{ABB}$ & $\mathrm{BBC}$ & B & BDD & BCC & $\mathrm{ABC}$ & B & B & BBD & 985 \\
\hline 122 & 145 & $\mathrm{~F}$ & 167047 & BBD & $\mathrm{ABB}$ & $\mathrm{ABB}$ & $\mathrm{BBC}$ & B & BDD & BCC & BCC & BBC & B & BBD & 967 \\
\hline 123 & 145 & $\mathrm{~F}$ & 167048 & BBD & $\mathrm{ABB}$ & $\mathrm{ABB}$ & $\mathrm{BBC}$ & B & BDD & BCC & $\mathrm{ABC}$ & B & B & BBD & 1110 \\
\hline 124 & 145 & $\mathrm{~F}$ & 167049 & BBD & $\mathrm{ABB}$ & $\mathrm{ABB}$ & $\mathrm{BBC}$ & B & BDD & BCC & $\mathrm{ABC}$ & B & B & BBD & FCM \\
\hline 125 & 145 & $\mathrm{~F}$ & 167050 & BBD & $\mathrm{ABB}$ & $\mathrm{ABB}$ & $\mathrm{BBC}$ & B & BDD & BCC & $\mathrm{ABC}$ & $\mathrm{BCC}$ & B & BBD & 878 \\
\hline 126 & 145 & $\mathrm{~F}$ & 167051 & BBD & $\mathrm{ABB}$ & B & $\mathrm{BBC}$ & B & BDD & BCC & $\mathrm{ABC}$ & BCC & B & BBD & 913 \\
\hline
\end{tabular}


APPENDIX 2-4

(Continued)

Locus

Site Sex AMNH Aat-1 Aat-2 Idh-1 Ldh-1 Ldh-2 Mdh-1 Mpi Pgi Pgm-1 Pgm-2 Sod-1 blood

\begin{tabular}{|c|c|c|c|c|c|c|c|c|c|c|c|c|c|c|c|}
\hline 127 & 145 & $\mathrm{~F}$ & 167052 & $\mathrm{BBD}$ & ABB & $\mathrm{ABB}$ & $\mathrm{BBC}$ & B & BDD & - & $\mathrm{ABC}$ & B & B & $\mathrm{BBD}$ & 1175 \\
\hline 128 & 145 & $\mathrm{~F}$ & 167053 & BBD & ABB & $\mathrm{ABB}$ & $\mathrm{BBC}$ & B & BDD & $\mathrm{BCC}$ & $\mathrm{AAC}$ & B & B & BBD & 909 \\
\hline 129 & 145 & F & 167054 & BBD & ABB & $\mathrm{ABB}$ & $\mathrm{BBC}$ & B & BDD & 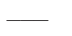 & $\mathrm{ABC}$ & B & B & BBD & 1120 \\
\hline 130) & 145 & $\mathrm{~F}$ & 167055 & $\mathrm{BBC}$ & $\mathrm{ABB}$ & $\mathrm{ABB}$ & $\mathrm{BBC}$ & B & BDD & - & $\mathrm{ACC}$ & B & B & BBD & 985 \\
\hline 131 & 148 & $\mathrm{~F}$ & 167012 & BBD & $\mathrm{ABB}$ & $\mathrm{ABB}$ & $\mathrm{BBC}$ & B & BDD & $\mathrm{BCC}$ & $\mathrm{BCC}$ & B & B & BDD & 1041 \\
\hline 132 & 148 & $\mathrm{~F}$ & 167013 & BBD & $\mathrm{ABB}$ & $\mathrm{ABB}$ & $\mathrm{BBC}$ & B & BDD & $\mathrm{BCC}$ & $\mathrm{BBC}$ & & & BBD & 984 \\
\hline 133 & 148 & $\mathrm{~F}$ & 167014 & BBD & $\mathrm{ABB}$ & $\mathrm{ABB}$ & $\mathrm{BBC}$ & B & BDD & $\mathrm{C}$ & $\mathrm{BCC}$ & B & B & BBD & 1021 \\
\hline 134 & 148 & $\mathrm{~F}$ & 167015 & BBD & $\mathrm{ABB}$ & $\mathrm{ABB}$ & $\mathrm{D}$ & B & BDD & $\mathrm{BCC}$ & $\mathrm{BBC}$ & - & & BBD & 977 \\
\hline 135 & 148 & $\mathrm{~F}$ & 167016 & BBD & $\mathrm{ABB}$ & B & $\mathrm{BBC}$ & B & $\mathrm{D}$ & BCC & $\mathrm{ABC}$ & $\mathrm{ABC}$ & B & BBD & 1180 \\
\hline 136 & 148 & $\mathrm{~F}$ & 167017 & BBD & $\mathrm{ABB}$ & $\mathrm{ABB}$ & $\mathrm{D}$ & $\mathrm{AAB}$ & BDD & $\mathrm{BCC}$ & $\mathrm{BCC}$ & - & 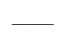 & BBD & 944 \\
\hline 137 & 148 & $\mathrm{~F}$ & 167018 & BBD & ABB & B & $\mathrm{BBC}$ & B & BDD & $\mathrm{BCC}$ & $\mathrm{BCC}$ & - & 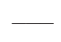 & BBD & 1126 \\
\hline 138 & 148 & F & 167019 & BBD & ABB & $\mathrm{ABB}$ & $\mathrm{BBC}$ & B & BDD & $\mathrm{BCC}$ & $\mathrm{BCC}$ & B & B & BBD & 1164 \\
\hline 139 & 149 & $\mathrm{~F}$ & 169446 & BBD & $\mathrm{ABB}$ & $\mathrm{ABB}$ & $\mathrm{BBC}$ & B & BBD & $\mathrm{BCC}$ & $\mathrm{AAC}$ & $\mathrm{BBC}$ & B & BBD & FCM \\
\hline 140) & 151 & $\mathrm{~F}$ & 169447 & BBD & $\mathrm{ABB}$ & $\mathrm{ABB}$ & $\mathrm{BBC}$ & B & BDD & $\mathrm{BCC}$ & $\mathrm{BBC}$ & $\mathrm{BBC}$ & B & BBD & FCM \\
\hline 141 & 151 & $\mathrm{~F}$ & 169448 & BBD & $\mathrm{ABB}$ & $\mathrm{ABB}$ & $\mathrm{BBC}$ & B & BDD & $\mathrm{BCC}$ & $\mathrm{AAC}$ & $\mathrm{BBC}$ & B & BBD & FCM \\
\hline 142 & 152 & M & 169449 & BBD & $\mathrm{ABB}$ & - & - & B & BDD & BCC & - & $\mathrm{BBC}$ & B & BBD & FCM \\
\hline 143 & 152 & $\mathrm{~F}$ & 169450 & BBD & ABB & - & $\mathrm{BBC}$ & ABB & BDD & $\mathrm{BBC}$ & - & $\mathrm{BBC}$ & B & BBD & FCM \\
\hline 144 & 155 & $\mathrm{~F}$ & 160285 & $\mathrm{BBD}$ & ABB & $\mathrm{ABB}$ & $\mathrm{BBC}$ & B & $\mathrm{BDD}$ & $\mathrm{BCC}$ & $\mathrm{ACC}$ & B & B & BBD & 1057 \\
\hline 145 & 155 & $\mathrm{~F}$ & 160286 & BBD & ABB & $\mathrm{ABB}$ & $\mathrm{BBC}$ & $\mathrm{ABB}$ & BDD & $\mathrm{BCC}$ & $\mathrm{ACC}$ & $\mathrm{BBC}$ & B & BBD & 1022 \\
\hline 146 & 155 & $\mathrm{~F}$ & 160287 & BBD & $\mathrm{ABB}$ & $\mathrm{ABB}$ & $\mathrm{BBC}$ & B & BDD & $\mathrm{BCC}$ & - & $\mathrm{BBC}$ & B & BBD & 1094 \\
\hline 147 & 155 & $\mathrm{~F}$ & 160288 & BBD & $\mathrm{ABB}$ & $\mathrm{ABB}$ & $\mathrm{BBC}$ & B & BDD & - & $\mathrm{ACC}$ & $\mathrm{BBC}$ & B & BBD & 1013 \\
\hline 148 & 155 & $\mathrm{~F}$ & 160289 & BBD & $\mathrm{ABB}$ & $\mathrm{ABB}$ & $\mathrm{BBC}$ & B & BDD & $\mathrm{BCC}$ & $\mathrm{ACC}$ & $\mathrm{BBC}$ & B & BBD & 951 \\
\hline 149 & 155 & $\mathrm{~F}$ & 160290 & BBD & ABB & B & $\mathrm{C}$ & B & BDD & $\mathrm{BCC}$ & QCC & - & B & BBD & 985 \\
\hline 150) & 155 & $\mathrm{~F}$ & 160291 & BBD & $\mathrm{ABB}$ & $\mathrm{ABB}$ & $\mathrm{BBC}$ & B & BDD & - & ACC & $\mathrm{BBC}$ & B & BBD & 876 \\
\hline 151 & 161 & $\mathrm{~F}$ & 166999 & BBD & ABB & $\mathrm{ABB}$ & $\mathrm{BBC}$ & B & BDD & $\mathrm{BCC}$ & - & B & B & BBD & 1043 \\
\hline 152 & 161 & $\mathrm{~F}$ & 167000 & $\mathrm{BBD}$ & ABB & $\mathrm{ABB}$ & $\mathrm{BBC}$ & B & $\mathrm{BDD}$ & $\mathrm{BCC}$ & - & B & B & BBD & 1067 \\
\hline 153 & 161 & $\mathrm{~F}$ & 167001 & - & - & - & - & - & $\mathrm{BDD}$ & $\mathrm{BCC}$ & - & B & B & BBD & 946 \\
\hline 154 & 162 & $\mathrm{~F}$ & 167002 & BBD & $\mathrm{ABB}$ & $\mathrm{ABB}$ & $\mathrm{BBC}$ & ABB & BDD & $\mathrm{BCC}$ & - & - & 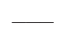 & BBD & 1078 \\
\hline 155 & 162 & $\mathrm{~F}$ & 167003 & BBD & $\mathrm{ABB}$ & $\mathrm{ABB}$ & $\mathrm{BBC}$ & B & BDD & $\mathrm{BCC}$ & - & - & - & BBD & 928 \\
\hline 156 & 162 & $\mathrm{~F}$ & 169359 & $\mathrm{BBD}$ & ABB & $\mathrm{ABB}$ & $\mathrm{BBC}$ & B & BDD & $\mathrm{BCC}$ & $\mathrm{ACC}$ & $\mathrm{BBC}$ & B & BBD & FCM \\
\hline 157 & 162 & $\mathrm{~F}$ & 169360 & $\mathrm{BBD}$ & $\mathrm{ABB}$ & $\mathrm{ABB}$ & $\mathrm{BBC}$ & B & BDD & $\mathrm{BCC}$ & $\mathrm{ACC}$ & $\mathrm{BC}$ & B & BBD & FCM \\
\hline 158 & 162 & $\mathrm{~F}$ & 169361 & BBD & $\mathrm{ABB}$ & $\mathrm{ABB}$ & $\mathrm{BBC}$ & B & BDD & $\mathrm{BCC}$ & $\mathrm{BCC}$ & B & B & BBD & FCM \\
\hline 159 & 162 & M & 169362 & BBD & ABB & $\mathrm{ABB}$ & $\mathrm{BBC}$ & $\mathrm{AAB}$ & BDD & $\mathrm{BCC}$ & $\mathrm{AAC}$ & $\mathrm{BBC}$ & B & BBD & FCM \\
\hline 160) & 162 & $\mathrm{~F}$ & 169363 & BBD & ABB & $\mathrm{ABB}$ & $\mathrm{BBC}$ & B & BDD & $\mathrm{BCC}$ & $\mathrm{ACC}$ & $\mathrm{BBC}$ & B & BBD & FCM \\
\hline 161 & 162 & $\mathrm{~F}$ & 169364 & BBD & $\mathrm{ABB}$ & $\mathrm{ABB}$ & $\mathrm{BBC}$ & B & BDD & $\mathrm{BCC}$ & - & B & B & BBD & FCM \\
\hline 162 & 162 & $\mathrm{~F}$ & 169365 & BBD & $\mathrm{ABB}$ & $\mathrm{ABB}$ & $\mathrm{BBC}$ & B & BDD & $\mathrm{BCC}$ & A & B & B & BBD & FCM \\
\hline 163 & 162 & $\mathrm{~F}$ & 169366 & BBD & $\mathrm{ABB}$ & $\mathrm{ABB}$ & $\mathrm{BBC}$ & B & BDD & $\mathrm{BCC}$ & $\mathrm{ACC}$ & $\mathrm{BBC}$ & B & BBD & FCM \\
\hline 164 & 168 & $\mathrm{~F}$ & 160292 & BBD & $\mathrm{ABB}$ & $\mathrm{ABB}$ & $\mathrm{BBC}$ & A & BDD & - & $\mathrm{AAC}$ & $\mathrm{BBC}$ & B & BBD & 1071 \\
\hline 165 & 168 & $\mathrm{~F}$ & 160293 & $\mathrm{BBD}$ & ABB & $\mathrm{ABB}$ & $\mathrm{BBC}$ & B & BDD & - & - & $\mathrm{BBC}$ & B & BBD & 1012 \\
\hline 166 & 168 & $\mathrm{~F}$ & 160294 & BBD & $\mathrm{ABB}$ & $\mathrm{ABB}$ & $\mathrm{BBC}$ & A & BDD & - & $\mathrm{AAC}$ & $\mathrm{BBC}$ & B & BBD & 1099 \\
\hline 167 & 168 & $\mathrm{~F}$ & 160295 & BBD & $\mathrm{ABB}$ & $\mathrm{ABB}$ & $\mathrm{BBC}$ & A & BDD & - & $\mathrm{ACC}$ & $\mathrm{BBC}$ & B & BBD & 1047 \\
\hline 168 & 168 & $\mathrm{~F}$ & 160296 & BBD & ABB & B & $\mathrm{BBC}$ & B & BDD & - & - & $\mathrm{BBC}$ & B & BBD & 962 \\
\hline 169 & 168 & $\mathrm{~F}$ & 160297 & BBD & $\mathrm{ABB}$ & $\mathrm{ABB}$ & $\mathrm{BBC}$ & B & BDD & - & - & $\mathrm{BBC}$ & B & BBD & 946 \\
\hline 170) & 168 & $\mathrm{~F}$ & 160298 & BBD & $\mathrm{ABB}$ & $\mathrm{ABB}$ & $\mathrm{BBC}$ & B & BDD & - & - & $\mathrm{BBC}$ & B & BBD & 982 \\
\hline 171 & 168 & $\mathrm{~F}$ & 160299 & BBD & - & $\mathrm{ABB}$ & $\mathrm{BBC}$ & B & BDD & - & - & $\mathrm{BBC}$ & B & BBD & 966 \\
\hline 172 & 168 & $\mathrm{~F}$ & 160300 & BBD & - & $\mathrm{ABB}$ & $\mathrm{BBC}$ & B & BDD & - & - & $\mathrm{BBC}$ & B & BBD & 905 \\
\hline 173 & 168 & $\mathrm{~F}$ & 160301 & BBD & - & B & $\mathrm{BBC}$ & B & BDD & - & - & $\mathrm{BBC}$ & B & BBD & 803 \\
\hline 174 & 168 & $\mathrm{~F}$ & 160302 & BBD & ABB & $\mathrm{ABB}$ & $\mathrm{BBC}$ & $\mathrm{AAB}$ & BDD & $\mathrm{BCC}$ & - & $\mathrm{BBC}$ & B & BBD & 946 \\
\hline 175 & 168 & $\mathrm{~F}$ & 160303 & BBD & $\mathrm{ABB}$ & $\mathrm{ABB}$ & $\mathrm{BBC}$ & $\mathrm{AAB}$ & BDD & - & $\mathrm{AAC}$ & $\mathrm{BBC}$ & B & BBD & 1062 \\
\hline 176 & 168 & $\mathrm{~F}$ & 160304 & BBD & ABB & $\mathrm{ABB}$ & $\mathrm{BBC}$ & $\mathrm{AAB}$ & $\mathrm{BDD}$ & $\mathrm{BCC}$ & - & $\mathrm{BBC}$ & B & BBD & 955 \\
\hline 177 & 168 & $\mathrm{~F}$ & 160305 & BBD & ABB & $\mathrm{ABB}$ & $\mathrm{BBC}$ & ABB & BDD & $\mathrm{BCC}$ & - & $\mathrm{BBC}$ & B & BBD & 911 \\
\hline 178 & 168 & $\mathrm{~F}$ & 160306 & BBD & $\mathrm{ABB}$ & $\mathrm{ABB}$ & $\mathrm{BBC}$ & $\mathrm{ABB}$ & BDD & $\mathrm{BCC}$ & - & $\mathrm{BBC}$ & B & BBD & 1007 \\
\hline
\end{tabular}


APPENDIX 2-4

(Continued)

\begin{tabular}{|c|c|c|c|c|c|c|c|c|c|c|c|c|c|c|c|}
\hline & \multirow[b]{2}{*}{ Site } & \multirow[b]{2}{*}{ Sex } & \multirow[b]{2}{*}{ AMNH } & \multicolumn{12}{|c|}{ Locus } \\
\hline & & & & Aat-1 & Aat-2 & Idh-1 & Ldh-1 & Ldh-2 & Mdh-1 & Mpi & Pgi & Pgm-1 & Pgm-2 & Sod-1 & blood \\
\hline 179 & 168 & $\mathrm{~F}$ & 160307 & BBD & ABB & B & B & B & BDD & BCC & - & BBC & B & BBD & 1058 \\
\hline 180) & 168 & $\mathrm{~F}$ & 160308 & BBD & $\mathrm{ABB}$ & $\mathrm{ABB}$ & $\mathrm{BBC}$ & $\mathrm{AAB}$ & BDD & $\mathrm{BCC}-$ & - & $\mathrm{BBC}$ & B & BBD & 1072 \\
\hline 181 & 168 & $\mathrm{~F}$ & 160309 & BBD & $\mathrm{ABB}$ & $\mathrm{ABB}$ & $\mathrm{BBC}$ & $\mathrm{ABB}$ & BDD & $\mathrm{BCC}$ & & BBC & B & BBD & 1070 \\
\hline 182 & 168 & $\mathrm{~F}$ & 160311 & BBD & $\mathrm{ABB}$ & $\mathrm{ABB}$ & $\mathrm{BBC}$ & $\mathrm{AAB}$ & BDD & -1 & $\mathrm{ACC}$ & $\mathrm{BBC}$ & B & BBD & 906 \\
\hline 183 & 168 & $\mathrm{~F}$ & 160312 & BBD & $\mathrm{ABB}$ & $\mathrm{ABB}$ & BBC & $\mathrm{ABB}$ & BDD & & ACC & $\mathrm{BBC}$ & B & BBD & 858 \\
\hline 184 & 168 & $\mathrm{~F}$ & 160313 & BBD & $\mathrm{ABB}$ & $\mathrm{ABB}$ & $\mathrm{BBC}$ & $\mathrm{AAB}$ & BDD & $\mathrm{BCC}-$ & & $\mathrm{BBC}$ & B & BBD & 931 \\
\hline 185 & 168 & $\mathrm{~F}$ & 160314 & BBD & $\mathrm{ABB}$ & $\mathrm{ABB}$ & $\mathrm{BBC}$ & $\mathrm{AAB}$ & BDD & $\mathrm{BCC}$ & $\mathrm{ACC}$ & $\mathrm{BBC}$ & B & BBD & 924 \\
\hline 186 & 168 & $\mathrm{~F}$ & 160315 & BBD & $\mathrm{ABB}$ & $\mathrm{ABB}$ & BBC & $\mathrm{ABB}$ & BDD & $\mathrm{BCC}$ & $\mathrm{ACC}$ & BBC & B & BBD & 976 \\
\hline 187 & 168 & $\mathrm{~F}$ & 160316 & BBD & $\mathrm{ABB}$ & $\mathrm{ABB}$ & $\mathrm{BBC}$ & B & BDD & & ACC & $\mathrm{BBC}$ & B & BBD & 898 \\
\hline 188 & 168 & $\mathrm{~F}$ & 160317 & BBD & ABB & $\mathrm{ABB}$ & BBC & B & BDD & -1 & ACC & BBC & B & BBD & 937 \\
\hline 189 & 168 & $\mathrm{~F}$ & 160320 & BBD & $\mathrm{ABB}$ & $\mathrm{ABB}$ & BBC & $\mathrm{AAB}$ & BDD & $\mathrm{BCC}$ & - & BBC & B & BBD & 1017 \\
\hline 190) & 174 & $\mathrm{~F}$ & 169926 & BBD & $\mathrm{ABB}$ & $\mathrm{ABB}$ & $\mathrm{BBC}$ & B & BDD & - & ACC & B & B & BBD & FCM \\
\hline 191 & 174 & $\mathrm{~F}$ & 169927 & BBD & ABB & $\mathrm{ABB}$ & BBC & B & BDD & & AAC & B & B & BBD & FCM \\
\hline 192 & 189 & $\mathrm{~F}$ & 166018 & BBD & $\mathrm{ABB}$ & $\mathrm{ABB}$ & BBC & B & BDD & $\mathrm{BCC}$ & C & B & B & BBD & 997 \\
\hline 193 & 189 & $\mathrm{~F}$ & 166019 & BBD & $\mathrm{ABB}$ & $\mathrm{ABB}$ & $\mathrm{BBC}$ & $\mathrm{ABB}$ & BDD & BCC & BCC & $\mathrm{BBC}$ & B & BBD & 986 \\
\hline 194 & 189 & $\mathrm{~F}$ & 166020 & BBD & ABB & $\mathrm{ABB}$ & D & B & BDD & $\mathrm{BCC}$ & AAC & BBC & B & BBD & 1032 \\
\hline 195 & 189 & $\mathrm{~F}$ & 166021 & BBD & $\mathrm{ABB}$ & $\mathrm{ABB}$ & BBC & $\mathrm{ABB}$ & BDD & $\mathrm{BCC}$ & BCC & BBC & B & BBD & 958 \\
\hline 196 & 189 & $\mathrm{~F}$ & 166022 & BBD & $\mathrm{ABB}$ & $\mathrm{ABB}$ & BBC & B & BDD & $\mathrm{BCC}$ & C & B & B & BBD & 969 \\
\hline 197 & 189 & $\mathrm{~F}$ & 166023 & BBD & $\mathrm{ABB}$ & $\mathrm{ABB}$ & BBC & B & BDD & $\mathrm{BCC}$ & $\mathrm{C}$ & B & B & BBD & 1004 \\
\hline 198 & 189 & $\mathrm{~F}$ & 166024 & BBD & $\mathrm{ABB}$ & $\mathrm{ABB}$ & BBC & B & BDD & $\mathrm{BCC}$ & $\mathrm{C}$ & $\mathrm{BBC}$ & B & BBD & 885 \\
\hline 199 & 189 & $\mathrm{~F}$ & 166025 & BBD & $\mathrm{ABB}$ & $\mathrm{ABB}$ & D & B & BDD & $\mathrm{BCC}$ & $\mathrm{C}$ & $\mathrm{BBC}$ & B & BBD & 894 \\
\hline 200) & 189 & $\mathrm{~F}$ & 166026 & BBD & $\mathrm{ABB}$ & $\mathrm{ABB}$ & $\mathrm{BBC}$ & B & BDD & $\mathrm{BCC}$ & $\mathrm{C}$ & BBC & B & BBD & 981 \\
\hline 201 & 189 & $\mathrm{~F}$ & 166027 & BBD & $\mathrm{ABB}$ & $\mathrm{ABB}$ & D & B & BDD & $\mathrm{BCC}$ & $\mathrm{BCC}$ & B & B & BBD & 817 \\
\hline 202 & 189 & $\mathrm{~F}$ & 166028 & BBD & $\mathrm{ABB}$ & $\mathrm{ABB}$ & $\mathrm{BBC}$ & $\mathrm{ABB}$ & BDD & $\mathrm{BCC}$ & AAC & BBC & B & BBD & 899 \\
\hline 203 & 189 & $\mathrm{~F}$ & 166029 & BBD & $\mathrm{ABB}$ & $\mathrm{ABB}$ & D & $\mathrm{ABB}$ & BDD & BCC & ACC & B & B & BBD & 842 \\
\hline 204 & 189 & $\mathrm{~F}$ & 166030 & BBD & $\mathrm{ABB}$ & $\mathrm{ABB}$ & D & B & BDD & $\mathrm{BCC}$ & $\mathrm{C}$ & BBC & B & BBD & 916 \\
\hline 205 & 189 & $\mathrm{~F}$ & 166031 & BBD & $\mathrm{ABB}$ & $\mathrm{ABB}$ & $\mathrm{BBC}$ & B & BDD & $\mathrm{BCC}$ & $\mathrm{C}$ & $\mathrm{BBC}$ & B & BBD & 999 \\
\hline 206 & 189 & $\mathrm{~F}$ & 166032 & BBD & ABB & $\mathrm{ABB}$ & $\mathrm{BBC}$ & B & BDD & C & C & $\mathrm{BBC}$ & B & BBD & 929 \\
\hline 207 & 189 & $\mathrm{~F}$ & 166033 & BBD & $\mathrm{ABB}$ & $\mathrm{ABB}$ & D & B & BDD & $\mathrm{BBC}$ & $\mathrm{ACC}$ & B & B & BBD & 958 \\
\hline 208 & 189 & $\mathrm{~F}$ & 166034 & BBD & $\mathrm{ABB}$ & $\mathrm{ABB}$ & D & B & BDD & $\mathrm{BCC}$ & $\mathrm{C}$ & $\mathrm{BBC}$ & B & BBD & - \\
\hline 209 & 189 & $\mathrm{~F}$ & 166035 & BBD & $\mathrm{ABB}$ & $\mathrm{ABB}$ & $\mathrm{BBC}$ & B & BDD & BCC & - & $\mathrm{BBC}$ & B & BBD & 925 \\
\hline 210) & 189 & $\mathrm{~F}$ & 166036 & BBD & $\mathrm{ABB}$ & $\mathrm{ABB}$ & BBC & B & BDD & $\mathrm{BCC}$ & - & $\mathrm{BBC}$ & B & BBD & 1198 \\
\hline 211 & 189 & $\mathrm{~F}$ & 166037 & BBD & $\mathrm{ABB}$ & $\mathrm{ABB}$ & $\mathrm{BBC}$ & B & BDD & $\mathrm{BCC}$ & - & $\mathrm{BBC}$ & B & BBD & 814 \\
\hline 212 & 197 & $\mathrm{~F}$ & 169343 & BBD & $\mathrm{ABB}$ & $\mathrm{ABB}$ & $\mathrm{BBC}$ & B & BDD & $\mathrm{BCC}$ & - & B & B & BBD & FCM \\
\hline 213 & 198 & $\mathrm{~F}$ & 169344 & BBD & $\mathrm{ABB}$ & $\mathrm{ABB}$ & $\mathrm{BBC}$ & $\mathrm{BBC}$ & BBD & $\mathrm{BCC}$ & AAC & B & B & BBD & FCM \\
\hline 214 & 198 & $\mathrm{~F}$ & 169345 & BBD & $\mathrm{ABB}$ & $\mathrm{ABB}$ & BBC & A & BBD & $\mathrm{BCC}$ & AAC & B & B & BBD & FCM \\
\hline 215 & 209 & $\mathrm{~F}$ & 160267 & BDD & $\mathrm{ABB}$ & $\mathrm{ABB}$ & $\mathrm{BBC}$ & QAA & BBD & BCC & AAC & B & B & BBD & 862 \\
\hline 216 & 211 & $\mathrm{~F}$ & 158824 & BBD & $\mathrm{ABB}$ & $\mathrm{ABB}$ & $\mathrm{BBC}$ & B & BDD & - & - & B & B & BBD & 1049 \\
\hline 217 & 211 & $\mathrm{~F}$ & 160256 & BBD & $\mathrm{ABB}$ & $\mathrm{ABB}$ & $\mathrm{BBC}$ & A & BDD & BCC & - & B & B & BBD & 1011 \\
\hline 218 & 214 & $\mathrm{~F}$ & 158811 & BBD & $\mathrm{ABB}$ & $\mathrm{ABB}$ & BBC & B & BDD & BCC & - & BBC & B & BBD & 1007 \\
\hline 219 & 215 & $\mathrm{~F}$ & 158813 & BBD & $\mathrm{ABB}$ & $\mathrm{ABB}$ & $\mathrm{BBC}$ & B & BDD & $\mathrm{BCC}$ & ACC & B & B & BBD & 956 \\
\hline 220) & 215 & $\mathrm{~F}$ & 158814 & BBD & $\mathrm{ABB}$ & $\mathrm{ABB}$ & $\mathrm{BBC}$ & B & BDD & BCC & ACC & & B & BBD & 960 \\
\hline 221 & 215 & $\mathrm{~F}$ & 158815 & BBD & $\mathrm{ABB}$ & $\mathrm{ABB}$ & $\mathrm{BBC}$ & B & BDD & $\mathrm{BCC}$ & ACC & B & B & BBD & 1043 \\
\hline 222 & 215 & $\mathrm{~F}$ & 158817 & BBD & ABB & $\mathrm{ABB}$ & BBC & B & BDD & BCC & ACC & B & B & BBD & 1234 \\
\hline 223 & 215 & $\mathrm{~F}$ & 158818 & BBD & $\mathrm{ABB}$ & $\mathrm{ABB}$ & $\mathrm{BBC}$ & B & BDD & BCC & ACC & B & B & BBD & 1076 \\
\hline 224 & 215 & $\mathrm{~F}$ & 158819 & BBD & $\mathrm{ABB}$ & $\mathrm{ABB}$ & $\mathrm{BBC}$ & B & BDD & $\mathrm{BCC}$ & ACC & B & B & BBD & 1027 \\
\hline 225 & 215 & $\mathrm{~F}$ & 158820 & BBD & $\mathrm{ABB}$ & $\mathrm{ABB}$ & BBC & B & BDD & $\mathrm{BCC}$ & $\mathrm{ACC}$ & B & B & BBD & 935 \\
\hline 226 & 215 & $\mathrm{~F}$ & 158821 & BBD & $\mathrm{ABB}$ & $\mathrm{ABB}$ & BBC & B & BDD & BCC & ACC & B & B & BBD & 987 \\
\hline 227 & 215 & $\mathrm{~F}$ & 158822 & BBD & $\mathrm{ABB}$ & $\mathrm{ABB}$ & $\mathrm{BBC}$ & B & BDD & BCC & ACC & B & B & BBD & 1179 \\
\hline 228 & 216 & $\mathrm{~F}$ & 153120 & BBD & $\mathrm{ABB}$ & $\mathrm{ABB}$ & $\mathrm{BCC}$ & B & BDD & - & - & B & B & BBD & 1048 \\
\hline
\end{tabular}


Appendix 2-5: Genotypes of triploid (3n) Ambystoma laterale - (2) jeffersonianum (LJJ) specimens ordered by site number.

\begin{tabular}{|c|c|c|c|c|c|c|c|c|c|c|c|c|c|c|c|}
\hline & \multirow[b]{2}{*}{ Site } & \multirow[b]{2}{*}{ Sex } & \multirow[b]{2}{*}{ AMNH } & \multicolumn{12}{|c|}{ Locus } \\
\hline & & & & Aat-1 & Aat-2 & Idh-1 & Ldh-1 & Ldh-2 & Mdh-1 & Mpi & Pgi & Pgm-1 & Pgm-2 & Sod-1 & blood \\
\hline 1 & 20 & $\mathrm{~F}$ & 169808 & BDD & $\mathrm{AAB}$ & $\mathrm{AAB}$ & $\mathrm{BCC}$ & B & BBD & $\mathrm{AAB}$ & - & $\mathrm{BBC}$ & B & BDD & $\mathrm{FCM}$ \\
\hline 2 & 20 & $\mathrm{~F}$ & 169809 & BDD & $\mathrm{AAB}$ & $\mathrm{AAB}$ & - & B & BBD & $\mathrm{AAB}$ & . & $\mathrm{BBC}$ & B & BDD & $\mathrm{FCM}$ \\
\hline 3 & 28 & $\mathrm{~F}$ & 158787 & BDD & $\mathrm{ABB}$ & $\mathrm{AAB}$ & $\mathrm{BCC}$ & B & $\mathrm{BBD}$ & $\mathrm{BBC}$ & & $\mathrm{BBC}$ & B & BDD & 982 \\
\hline 4 & 108 & $\mathrm{~F}$ & 153118 & BDD & $\mathrm{AAB}$ & $\mathrm{AAB}$ & $\mathrm{BCC}$ & $\mathrm{ABB}$ & BBD & - & - & $\mathrm{BCC}$ & B & BDD & 1144 \\
\hline 5 & 108 & $\mathrm{~F}$ & 160330 & BDD & $\mathrm{AAB}$ & $\mathrm{AAB}$ & $\mathrm{BCC}$ & A & BBD & - & $\mathrm{ABC}$ & $\mathrm{BCC}$ & B & BDD & 1009 \\
\hline 6 & 109 & $\mathrm{~F}$ & 169849 & BDD & $\mathrm{AAB}$ & $\mathrm{AAB}$ & $\mathrm{BCC}$ & B & BBD & - & $\mathrm{ABC}$ & $\mathrm{BCC}$ & B & BDD & FCM \\
\hline 7 & 109 & $\mathrm{~F}$ & 169919 & BDD & $\mathrm{AAB}$ & $\mathrm{ABB}$ & $\mathrm{BCC}$ & B & $\mathrm{BBD}$ & - & $\mathrm{BBC}$ & $\mathrm{BCC}$ & B & BDD & FCM \\
\hline 8 & 109 & $\mathrm{~F}$ & 169920 & BDD & $\mathrm{AAB}$ & $\mathrm{ABB}$ & $\mathrm{BCC}$ & B & BBD & & $\mathrm{BBC}$ & $\mathrm{BCC}$ & B & BDD & $\mathrm{FCM}$ \\
\hline 9 & 109 & $\mathrm{~F}$ & 169921 & BDD & $\mathrm{AAB}$ & $\mathrm{AAB}$ & $\mathrm{BCC}$ & B & BBD & - & $\mathrm{ABC}$ & $\mathrm{BCC}$ & B & BDD & $\mathrm{FCM}$ \\
\hline 10) & 113 & $\mathrm{~F}$ & 162939 & BDD & $\mathrm{AAB}$ & $\mathrm{ABB}$ & $\mathrm{BCC}$ & B & BBD & $\mathrm{BBC}$ & - & B & B & BDD & 917 \\
\hline 11 & 114 & $\mathrm{~F}$ & 153112 & BDD & $\mathrm{AB}$ & $\mathrm{ABB}$ & BCC & B & BBD & - & - & $\mathrm{BBC}$ & B & BDD & - \\
\hline 12 & 115 & $\mathrm{~F}$ & 153116 & BDD & $\mathrm{AAB}$ & $\mathrm{AAB}$ & $\mathrm{BCC}$ & B & $\mathrm{BBD}$ & - & $\mathrm{AAB}$ & $\mathrm{BBC}$ & B & BDD & 948 \\
\hline 13 & 115 & $\mathrm{~F}$ & 160337 & BDD & $\mathrm{AAB}$ & $\mathrm{AAB}$ & $\mathrm{BCC}$ & A & BBD & $\mathrm{AAB}$ & $\mathrm{AAC}$ & $\mathrm{BBC}$ & B & BDD & 1090 \\
\hline 14 & 117 & $\mathrm{~F}$ & 169451 & BDD & $\mathrm{AAB}$ & A & $\mathrm{BCC}$ & B & BBD & $\mathrm{BBC}$ & $\mathrm{AAC}$ & $\mathrm{BBC}$ & B & BDD & FCM \\
\hline 15 & 117 & $\mathrm{~F}$ & 169452 & BDD & $\mathrm{AAB}$ & A & $\mathrm{BCC}$ & B & BBD & $\mathrm{BBC}$ & AAC & $\mathrm{BBC}$ & B & BDD & FCM \\
\hline 16 & 117 & $\mathrm{~F}$ & 169453 & BDD & $\mathrm{AAB}$ & $\mathrm{ABB}$ & $\mathrm{BCC}$ & B & BBD & $\mathrm{BBC}$ & $\mathrm{ABC}$ & $\mathrm{BBC}$ & B & BDD & $\mathrm{FCM}$ \\
\hline 17 & 117 & $\mathrm{~F}$ & 169454 & BDD & $\mathrm{AAB}$ & A & $\mathrm{BCC}$ & B & BBD & $\mathrm{BBC}$ & $\mathrm{AAC}$ & $\mathrm{BBC}$ & B & BDD & $\mathrm{FCM}$ \\
\hline 18 & 117 & $\mathrm{~F}$ & 169455 & BDD & $\mathrm{AAB}$ & A & $\mathrm{BCC}$ & B & BBD & $\mathrm{BBC}$ & $\mathrm{AAC}$ & $\mathrm{BBC}$ & B & BDD & FCM \\
\hline 19 & 119 & $\mathrm{~F}$ & 160331 & BDD & $\mathrm{AAB}$ & $\mathrm{AAB}$ & $\mathrm{BCC}$ & $\mathrm{ABB}$ & BBD & $\mathrm{BBC}$ & - & BCC & B & BDD & 1239 \\
\hline 20) & 119 & $\mathrm{~F}$ & 160332 & BDD & $\mathrm{AAB}$ & $\mathrm{AAB}$ & $\mathrm{BCC}$ & $\mathrm{ABB}$ & BBD & $\mathrm{BBC}$ & - & $\mathrm{BCC}$ & B & BDD & 1148 \\
\hline 21 & 119 & $\mathrm{~F}$ & 160333 & BDD & $\mathrm{AAB}$ & $\mathrm{AAB}$ & $\mathrm{BCC}$ & $\mathrm{ABB}$ & $\mathrm{BBD}$ & $\mathrm{BBC}$ & - & $\mathrm{BCC}$ & B & BDD & 1503 \\
\hline 22 & 119 & $\mathrm{~F}$ & 160334 & BDD & $\mathrm{AAB}$ & $\mathrm{AAB}$ & $\mathrm{BCC}$ & $\mathrm{ABB}$ & BBD & - & $\mathrm{ABC}$ & $\mathrm{BCC}$ & B & BDD & 1321 \\
\hline 23 & 119 & $\mathrm{~F}$ & 160335 & BDD & $\mathrm{AAB}$ & $\mathrm{AAB}$ & $\mathrm{BCC}$ & $\mathrm{ABB}$ & BBD & $\mathrm{BBC}$ & - & $\mathrm{BCC}$ & B & BDD & 1358 \\
\hline 24 & 119 & $\mathrm{~F}$ & 160336 & BDD & $\mathrm{AAB}$ & $\mathrm{AAB}$ & $\mathrm{BCC}$ & $\mathrm{ABB}$ & BBD & - & $\mathrm{ABC}$ & $\mathrm{BBC}$ & B & BDD & 1283 \\
\hline 25 & 122 & $\mathrm{~F}$ & 153113 & BDD & $\mathrm{AAB}$ & $\mathrm{ABB}$ & $\mathrm{BCC}$ & B & BBD & - & $\mathrm{ABC}$ & B & B & BDD & 996 \\
\hline 26 & 122 & $\mathrm{~F}$ & 153114 & BDD & $\mathrm{AAB}$ & $\mathrm{AAB}$ & $\mathrm{BCC}$ & B & $\mathrm{BBD}$ & - & - & $\mathrm{BBC}$ & B & BDD & 937 \\
\hline 27 & 122 & $\mathrm{~F}$ & 153115 & BDD & $\mathrm{AAB}$ & $\mathrm{AAB}$ & $\mathrm{BCC}$ & B & BBD & - & - & $\mathrm{BBC}$ & B & BDD & 924 \\
\hline 28 & 123 & $\mathrm{~F}$ & 162835 & BDD & $\mathrm{AAB}$ & $\mathrm{AAB}$ & $\mathrm{BCC}$ & B & BBD & - & - & B & B & BDD & 713 \\
\hline 29 & 124 & $\mathrm{~F}$ & 169816 & BDD & $\mathrm{AAB}$ & $\mathrm{AAB}$ & $\mathrm{BCC}$ & B & BBD & $\mathrm{AAB}$ & - & $\mathrm{BBC}$ & B & BDD & FCM \\
\hline 30) & 124 & $\mathrm{~F}$ & 169817 & BDD & $\mathrm{AAB}$ & $\mathrm{AAB}$ & $\mathrm{BCC}$ & B & BBD & $\mathrm{AAB}$ & - & B & B & BDD & FCM \\
\hline 31 & 124 & $\mathrm{~F}$ & 169818 & BDD & $\mathrm{AAB}$ & $\mathrm{AAB}$ & $\mathrm{BCC}$ & B & BBD & $\mathrm{BBC}$ & AAC & $\mathrm{BBC}$ & B & BDD & FCM \\
\hline 32 & 124 & $\mathrm{~F}$ & 169819 & BDD & $\mathrm{AAB}$ & $\mathrm{AAB}$ & $\mathrm{BCC}$ & B & BBD & $\mathrm{BBC}$ & $\mathrm{ABC}$ & B & B & BDD & $\mathrm{FCM}$ \\
\hline 33 & 124 & $\mathrm{~F}$ & 169820 & BDD & $\mathrm{AAB}$ & $\mathrm{AAB}$ & $\mathrm{BCC}$ & B & BBD & $\mathrm{BBC}$ & - & $\mathrm{BBC}$ & B & BDD & FCM \\
\hline 34 & 124 & $\mathrm{~F}$ & 169821 & BDD & $\mathrm{AAB}$ & $\mathrm{AAB}$ & $\mathrm{BCC}$ & B & $\mathrm{BBD}$ & $\mathrm{BBC}$ & $\mathrm{BCC}$ & $\mathrm{BBC}$ & B & BDD & FCM \\
\hline 35 & 124 & $\mathrm{~F}$ & 169822 & BDD & $\mathrm{AAB}$ & $\mathrm{AAB}$ & $\mathrm{BCC}$ & B & BBD & $\mathrm{BBC}$ & $\mathrm{AAC}$ & $\mathrm{BBC}$ & B & BDD & FCM \\
\hline 36 & 126 & $\mathrm{~F}$ & 169777 & BDD & $\mathrm{AAB}$ & $\mathrm{AAB}$ & $\mathrm{BCC}$ & B & BBD & $\mathrm{BBC}$ & - & $\mathrm{BCC}$ & B & BDD & FCM \\
\hline 37 & 126 & $\mathrm{~F}$ & 169778 & BDD & $\mathrm{AAB}$ & $\mathrm{AAB}$ & $\mathrm{BCC}$ & B & BBD & $\mathrm{BBC}$ & - & $\mathrm{BBC}$ & B & BDD & FCM \\
\hline 38 & 126 & $\mathrm{~F}$ & 169779 & BDD & $\mathrm{AAB}$ & $\mathrm{AAB}$ & $\mathrm{BCC}$ & B & $\mathrm{BBD}$ & $\mathrm{BBC}$ & - & $\mathrm{BBC}$ & B & BDD & FCM \\
\hline 39 & 126 & $\mathrm{~F}$ & 169780 & BDD & $\mathrm{AAB}$ & $\mathrm{AAB}$ & $\mathrm{BCC}$ & B & BBD & $\mathrm{BBC}$ & - & $\mathrm{BBC}$ & B & BDD & FCM \\
\hline 40) & 126 & $\mathrm{~F}$ & 169781 & BDD & $\mathrm{AAB}$ & $\mathrm{AAB}$ & $\mathrm{BCC}$ & B & BBD & $\mathrm{BBC}$ & - & $\mathrm{BBC}$ & B & BDD & FCM \\
\hline 41 & 126 & $\mathrm{~F}$ & 169782 & BDD & $\mathrm{AAB}$ & $\mathrm{AAB}$ & $\mathrm{BCC}$ & B & BBD & $\mathrm{BBC}$ & - & $\mathrm{BBC}$ & B & BDD & $\mathrm{FCM}$ \\
\hline 42 & 126 & $\mathrm{~F}$ & 169783 & BDD & $\mathrm{AAB}$ & $\mathrm{ABB}$ & $\mathrm{BCC}$ & B & BBD & $\mathrm{BBC}$ & - & $\mathrm{BBC}$ & B & BDD & FCM \\
\hline 43 & 126 & $\mathrm{~F}$ & 169784 & BDD & $\mathrm{AAB}$ & $\mathrm{AAB}$ & $\mathrm{BCC}$ & B & $\mathrm{BBD}$ & $\mathrm{BBC}$ & - & $\mathrm{BBC}$ & B & BDD & $\mathrm{FCM}$ \\
\hline 44 & 126 & $\mathrm{~F}$ & 169785 & BDD & $\mathrm{AAB}$ & $\mathrm{AAB}$ & $\mathrm{BCC}$ & B & $\mathrm{BBD}$ & $\mathrm{BBC}$ & - & $\mathrm{BBC}$ & B & BDD & FCM \\
\hline 45 & 126 & $\mathrm{~F}$ & 169786 & BDD & $\mathrm{AAB}$ & $\mathrm{AAB}$ & $\mathrm{BCC}$ & B & BBD & $\mathrm{BBC}$ & - & $\mathrm{BCC}$ & B & BDD & FCM \\
\hline 46 & 126 & $\mathrm{~F}$ & 169787 & BDD & $\mathrm{AAB}$ & $\mathrm{AAB}$ & $\mathrm{BCC}$ & B & $\mathrm{BBD}$ & $\mathrm{BBC}$ & - & $\mathrm{BBC}$ & B & BDD & FCM \\
\hline 47 & 126 & $\mathrm{~F}$ & 169788 & BDD & $\mathrm{AAB}$ & $\mathrm{ABB}$ & $\mathrm{BCC}$ & B & BBD & BBD & AAC & $\mathrm{BCC}$ & B & BDD & FCM \\
\hline 48 & 126 & $\mathrm{~F}$ & 169789 & BDD & $\mathrm{AAB}$ & $\mathrm{AAB}$ & $\mathrm{BCC}$ & B & BBD & $\mathrm{BBC}$ & - & $\mathrm{BBC}$ & B & BDD & $\mathrm{FCM}$ \\
\hline 49 & 126 & $\mathrm{~F}$ & 169790 & BDD & $\mathrm{AAB}$ & $\mathrm{AAB}$ & $\mathrm{BCC}$ & B & BBD & $\mathrm{BBC}$ & - & $\mathrm{BBC}$ & B & BDD & FCM \\
\hline 50) & 126 & $\mathrm{~F}$ & 169791 & BDD & $\mathrm{AAB}$ & $\mathrm{AAB}$ & $\mathrm{BCC}$ & B & $\mathrm{BBD}$ & $\mathrm{BBC}$ & $\mathrm{ABC}$ & $\mathrm{BBC}$ & B & BDD & FCM \\
\hline 51 & 126 & $\mathrm{~F}$ & 169792 & BDD & $\mathrm{AAB}$ & $\mathrm{AAB}$ & $\mathrm{BCC}$ & $\mathrm{ABB}$ & BBD & $\mathrm{BBC}$ & $\mathrm{AAC}$ & $\mathrm{BCC}$ & B & BDD & FCM \\
\hline
\end{tabular}


APPENDIX 2-5

(Continued)

\begin{tabular}{|c|c|c|c|c|c|c|c|c|c|c|c|c|c|c|c|}
\hline & \multirow[b]{2}{*}{ Site } & \multirow[b]{2}{*}{ Sex } & \multirow[b]{2}{*}{ AMNH } & \multicolumn{12}{|c|}{ Locus } \\
\hline & & & & Aat-1 & Aat-2 & Idh-1 & Ldh-1 & Ldh-2 & Mdh-1 & Mpi & Pgi & Pgm-1 & Pgm-2 & Sod-1 & blood \\
\hline 52 & 126 & F & 169793 & BDD & AAB & $\mathrm{ABB}$ & $\mathrm{BCC}$ & B & BBD & BBD & AAC & $\mathrm{BBC}$ & B & BDD & $\mathrm{FCM}$ \\
\hline 53 & 126 & $\mathrm{~F}$ & 169794 & BDD & AAB & $\mathrm{AAB}$ & $\mathrm{BBC}$ & B & BBD & $\mathrm{BBC}$ & AAC & $\mathrm{BBC}$ & B & BDD & FCM \\
\hline 54 & 130 & $\mathrm{~F}$ & 162938 & BDD & AAB & QAA & $\mathrm{BCC}$ & B & BBD & $\mathrm{BBC}$ & $\mathrm{ABC}$ & $\mathrm{BBC}$ & B & BDD & 1027 \\
\hline 55 & 130 & $\mathrm{~F}$ & 165953 & BDD & $\mathrm{AAB}$ & $\mathrm{AAB}$ & $\mathrm{BCC}$ & B & BBD & $\mathrm{BBC}$ & $\mathrm{C}$ & $\mathrm{BCC}$ & B & BDD & 1032 \\
\hline 56 & 130 & $\mathrm{~F}$ & 165954 & BDD & $\mathrm{AAB}$ & QAA & $\mathrm{BCC}$ & B & BBD & $\mathrm{BBC}$ & C & $\mathrm{BCC}$ & B & BDD & 1115 \\
\hline 57 & 130 & $\mathrm{~F}$ & 165955 & BDD & $\mathrm{AAB}$ & QAB & $\mathrm{BCC}$ & B & BBD & $\mathrm{BBC}$ & 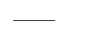 & BCC & B & BDD & 1037 \\
\hline 58 & 130 & $\mathrm{~F}$ & 165956 & BDD & $\mathrm{AAB}$ & $\mathrm{AAB}$ & $\mathrm{BCC}$ & B & BBD & $\mathrm{BBC}$ & 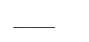 & $\mathrm{BBC}$ & B & BDD & 907 \\
\hline 59 & 130 & $\mathrm{~F}$ & 165957 & BDD & AAB & $\mathrm{AAB}$ & $\mathrm{BCC}$ & B & BBD & $\mathrm{BBC}$ & - & $\mathrm{BBC}$ & B & BDD & 857 \\
\hline 60) & 131 & $\mathrm{~F}$ & 165958 & BDD & $\mathrm{AAB}$ & QAB & $\mathrm{BCC}$ & B & BBD & $\mathrm{BBC}$ & & BCC & B & BDD & 1020 \\
\hline 61 & 131 & $\mathrm{~F}$ & 165959 & BDD & AAB & $\mathrm{AAB}$ & $\mathrm{BCC}$ & B & BBD & & $\mathrm{C}$ & $\mathrm{BBC}$ & B & BDD & 996 \\
\hline 62 & 131 & $\mathrm{~F}$ & 165960 & BDD & AAB & $\mathrm{AAB}$ & $\mathrm{BCC}$ & B & BBD & $\mathrm{BBC}$ & & BBC & B & BDD & 936 \\
\hline 63 & 131 & $\mathrm{~F}$ & 165961 & BDD & $\mathrm{AAB}$ & $\mathrm{ABB}$ & $\mathrm{BCC}$ & B & BBD & - & C & $\mathrm{BBC}$ & B & BDD & 841 \\
\hline 64 & 131 & $\mathrm{~F}$ & 165962 & BDD & AAB & QAB & $\mathrm{BCC}$ & B & BBD & - & C & BCC & B & BDD & 1042 \\
\hline 65 & 137 & $\mathrm{~F}$ & 167067 & BDD & $\mathrm{AAB}$ & $\mathrm{AAB}$ & $\mathrm{BCC}$ & B & BBD & $\mathrm{BBC}$ & 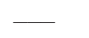 & B & B & BDD & 1031 \\
\hline 66 & 138 & $\mathrm{~F}$ & 167071 & BDD & $\mathrm{AAB}$ & - & $\mathrm{BCC}$ & B & BBD & $\mathrm{BBC}$ & - & B & B & BDD & 1058 \\
\hline 67 & 139 & $\mathrm{~F}$ & 167072 & BDD & AAB & $\mathrm{AAB}$ & $\mathrm{BCC}$ & B & BBD & $\mathrm{BBC}$ & AAC & $\mathrm{BBC}$ & B & BDD & 996 \\
\hline 68 & 140 & $\mathrm{~F}$ & 169998 & BDD & AAB & $\mathrm{AAB}$ & $\mathrm{BCC}$ & B & BBD & $\mathrm{BBC}$ & AAC & $\mathrm{BBC}$ & B & BDD & FMC \\
\hline 69 & 140 & $\mathrm{~F}$ & 170000 & BDD & $\mathrm{AAB}$ & $\mathrm{AAB}$ & $\mathrm{BCC}$ & B & BBD & $\mathrm{BBC}$ & AAC & $\mathrm{BBC}$ & B & BDD & FMC \\
\hline 70) & 140 & $\mathrm{~F}$ & 170001 & BDD & AAB & $\mathrm{AAB}$ & BCC & B & BBD & $\mathrm{BBC}$ & AAC & $\mathrm{BBC}$ & B & BDD & FMC \\
\hline 71 & 141 & $\mathrm{~F}$ & 170002 & BDD & $\mathrm{AAB}$ & $\mathrm{AAB}$ & $\mathrm{BCC}$ & B & BBD & $\mathrm{BBC}$ & $\mathrm{AAC}$ & $\mathrm{BBC}$ & B & BDD & FMC \\
\hline 72 & 141 & $\mathrm{~F}$ & 170003 & BDD & $\mathrm{AAB}$ & $\mathrm{AAB}$ & $\mathrm{BCC}$ & B & BBD & $\mathrm{BBC}$ & $\mathrm{AAC}$ & $\mathrm{BBC}$ & B & BDD & FMC \\
\hline 73 & 141 & $\mathrm{~F}$ & 170004 & BDD & $\mathrm{AAB}$ & $\mathrm{AAB}$ & $\mathrm{BCC}$ & B & BBD & $\mathrm{BBC}$ & AAC & $\mathrm{BBC}$ & B & BDD & FMC \\
\hline 74 & 142 & $\mathrm{~F}$ & 170005 & BDD & $\mathrm{AAB}$ & $\mathrm{AAB}$ & $\mathrm{BCC}$ & B & BBD & $\mathrm{BBC}$ & AAC & $\mathrm{BBC}$ & B & BDD & FMC \\
\hline 75 & 156 & $\mathrm{~F}$ & 162925 & BDD & $\mathrm{AAB}$ & $\mathrm{AAB}$ & $\mathrm{BCC}$ & B & BBD & $\mathrm{BBC}$ & - & $\mathrm{BCC}$ & B & BDD & 1147 \\
\hline 76 & 156 & $\mathrm{~F}$ & 162933 & BDD & $\mathrm{AAB}$ & $\mathrm{AAB}$ & $\mathrm{BCC}$ & B & BBD & $\mathrm{BBC}$ & AAC & $\mathrm{BCC}$ & B & BDD & 1048 \\
\hline 77 & 156 & $\mathrm{~F}$ & 162934 & BDD & $\mathrm{AAB}$ & $\mathrm{AAB}$ & $\mathrm{BCC}$ & B & BBD & $\mathrm{BBC}$ & AAC & BCC & B & BDD & 1035 \\
\hline 78 & 156 & $\mathrm{~F}$ & 162935 & BDD & $\mathrm{AAB}$ & $\mathrm{AAB}$ & $\mathrm{BCC}$ & B & BBD & $\mathrm{BBC}$ & AAC & $\mathrm{BCC}$ & B & BDD & 1114 \\
\hline 79 & 157 & $\mathrm{~F}$ & 162926 & BDD & $\mathrm{AAB}$ & $\mathrm{AAB}$ & $\mathrm{BBC}$ & B & BBD & $\mathrm{BBC}$ & - & $\mathrm{BBC}$ & B & BDD & \\
\hline 80) & 157 & $\mathrm{~F}$ & 162927 & BDD & $\mathrm{AAB}$ & $\mathrm{AAB}$ & $\mathrm{BCC}$ & B & BBD & $\mathrm{BBC}$ & - & $\mathrm{BBC}$ & B & BDD & 1156 \\
\hline 81 & 157 & $\mathrm{~F}$ & 162928 & BDD & $\mathrm{AAB}$ & $\mathrm{AAB}$ & $\mathrm{BCC}$ & B & BBD & $\mathrm{BBC}$ & - & $\mathrm{BCC}$ & B & BDD & 1385 \\
\hline 82 & 157 & $\mathrm{~F}$ & 162929 & BDD & $\mathrm{AAB}$ & $\mathrm{AAB}$ & $\mathrm{BCC}$ & $\mathrm{AAB}$ & BBD & $\mathrm{BBC}$ & - & $\mathrm{BBC}$ & B & BDD & 1143 \\
\hline 83 & 157 & $\mathrm{~F}$ & 162930 & BDD & $\mathrm{AAB}$ & $\mathrm{AAB}$ & $\mathrm{BBC}$ & B & BBD & $\mathrm{BBC}$ & $\mathrm{ABC}$ & $\mathrm{BCC}$ & B & BDD & 1084 \\
\hline 84 & 157 & $\mathrm{~F}$ & 162931 & BDD & $\mathrm{AAB}$ & $\mathrm{AAB}$ & $\mathrm{BCC}$ & B & BBD & $\mathrm{BBC}$ & - & $\mathrm{BCC}$ & B & BDD & 1144 \\
\hline 85 & 157 & $\mathrm{~F}$ & 162932 & BDD & $\mathrm{AAB}$ & $\mathrm{AAB}$ & $\mathrm{BCC}$ & B & BBD & - & ACC & BCC & B & BDD & 1235 \\
\hline 86 & 158 & $\mathrm{~F}$ & 165963 & BDD & $\mathrm{AAB}$ & $\mathrm{AAB}$ & $\mathrm{BCC}$ & B & BBD & $\mathrm{BBC}$ & - & BCC & B & BDD & 976 \\
\hline 87 & 158 & $\mathrm{~F}$ & 165964 & BDD & $\mathrm{AAB}$ & $\mathrm{AAB}$ & $\mathrm{BCC}$ & B & BBD & $\mathrm{BBC}$ & - & BCC & B & BDD & 984 \\
\hline 88 & 158 & $\mathrm{~F}$ & 165965 & BDD & $\mathrm{AAB}$ & $\mathrm{AAB}$ & $\mathrm{BCC}$ & B & BBD & $\mathrm{BBC}$ & - & BCC & B & BDD & 1043 \\
\hline 89 & 158 & $\mathrm{~F}$ & 165966 & BDD & $\mathrm{AAB}$ & $\mathrm{AAB}$ & $\mathrm{BBC}$ & B & BDD & $\mathrm{BCC}$ & - & $\mathrm{BBC}$ & B & BDD & 731 \\
\hline 90) & 158 & F & 165967 & BDD & $\mathrm{AAB}$ & $\mathrm{ABB}$ & $\mathrm{BBC}$ & B & ABD & $\mathrm{BBC}$ & - & $\mathrm{BBC}$ & B & BDD & 744 \\
\hline 91 & 159 & $\mathrm{~F}$ & 160322 & BDD & AAB & $\mathrm{AAB}$ & C & B & BBD & $\mathrm{BBC}$ & $\mathrm{ABC}$ & $\mathrm{BBC}$ & B & BDD & 965 \\
\hline 92 & 159 & $\mathrm{~F}$ & 160323 & BDD & - & $\mathrm{AAB}$ & BCC & B & BBD & - & AAC & - & B & BDD & 1000 \\
\hline 93 & 159 & $\mathrm{~F}$ & 160324 & BDD & $\mathrm{AAB}$ & $\mathrm{AAB}$ & $\mathrm{BCC}$ & & BBD & - & - & $\mathrm{BBC}$ & B & BDD & 925 \\
\hline 94 & 159 & $\mathrm{~F}$ & 162922 & BDD & $\mathrm{AAB}$ & $\mathrm{AAB}$ & BCC & B & BBD & $\mathrm{BBC}$ & AAC & BCC & B & BDD & 1031 \\
\hline 95 & 159 & $\mathrm{~F}$ & 162923 & BDD & $\mathrm{AAB}$ & $\mathrm{AAB}$ & $\mathrm{BCC}$ & B & BBD & $\mathrm{BBC}$ & AAC & $\mathrm{BBC}$ & B & BDD & 1069 \\
\hline 96 & 159 & $\mathrm{~F}$ & 162924 & BDD & $\mathrm{AAB}$ & $\mathrm{AAB}$ & $\mathrm{BCC}$ & B & BBD & $\mathrm{BBC}$ & - & $\mathrm{BBC}$ & B & BDD & 989 \\
\hline 97 & 160 & $\mathrm{~F}$ & 165968 & BDD & $\mathrm{AAB}$ & A & $\mathrm{BCC}$ & B & BBD & $\mathrm{BBC}$ & . & BCC & B & BDD & \\
\hline 98 & 160 & $\mathrm{~F}$ & 165969 & BDD & $\mathrm{AAB}$ & $\mathrm{AAB}$ & $\mathrm{BCC}$ & B & BBD & $\mathrm{BBC}$ & 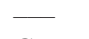 & BCC & B & BDD & 1129 \\
\hline 99 & 160 & $\mathrm{~F}$ & 165970 & BDD & $\mathrm{AAB}$ & $\mathrm{AAB}$ & $\mathrm{BCC}$ & B & BBD & - & C & BCC & B & BDD & 989 \\
\hline 100) & 160 & $\mathrm{~F}$ & 165971 & BDD & $\mathrm{AAB}$ & $\mathrm{AAB}$ & $\mathrm{BBC}$ & B & BBD & - & C & BCC & B & BDD & 1086 \\
\hline 101 & 161 & $\mathrm{~F}$ & 166991 & BDD & $\mathrm{AAB}$ & $\mathrm{AAB}$ & $\mathrm{BCC}$ & B & BBD & $\mathrm{BBC}$ & $\mathrm{BBC}$ & BCC & B & BDD & 1133 \\
\hline 102 & 161 & $\mathrm{~F}$ & 166992 & BDD & $\mathrm{AAB}$ & $\mathrm{AAB}$ & $\mathrm{BCC}$ & B & BBD & $\mathrm{BBC}$ & $\mathrm{ABB}$ & BCC & B & BDD & 1397 \\
\hline 103 & 161 & $\mathrm{~F}$ & 166993 & BDD & AAB & $\mathrm{AAB}$ & $\mathrm{BBC}$ & B & BBD & $\mathrm{BBC}$ & - & $\mathrm{C}$ & B & BDD & 1142 \\
\hline
\end{tabular}


APPENDIX 2-5

(Continued)

\begin{tabular}{|c|c|c|c|c|c|c|c|c|c|c|c|c|c|c|c|}
\hline & \multirow[b]{2}{*}{ Site } & \multirow[b]{2}{*}{ Sex } & \multirow[b]{2}{*}{ AMNH } & \multicolumn{12}{|c|}{ Locus } \\
\hline & & & & Aat-1 & Aat-2 & Idh-1 & Ldh-1 & Ldh-2 & Mdh-1 & Mpi & Pgi & Pgm-1 & Pgm-2 & Sod-1 & blood \\
\hline 104 & 161 & $\mathrm{~F}$ & 166994 & BDD & $\mathrm{AAB}$ & $\mathrm{AAB}$ & $\mathrm{BCC}$ & B & BBD & $\mathrm{BBC}$ & - & $\mathrm{BBC}$ & B & BDD & 1084 \\
\hline 105 & 161 & $\mathrm{~F}$ & 166995 & BDD & $\mathrm{AAB}$ & $\mathrm{AAB}$ & $\mathrm{BCC}$ & B & BBD & $\mathrm{BBC}$ & & $\mathrm{BCC}$ & B & BDD & 1021 \\
\hline 106 & 161 & F & 166996 & BDD & $\mathrm{AAB}$ & $\mathrm{ABB}$ & $\mathrm{BCC}$ & B & BBD & $\mathrm{BBC}$ & $\mathrm{BBC}$ & BBC & B & BDD & 1134 \\
\hline 107 & 161 & $\mathrm{~F}$ & 166997 & BDD & $\mathrm{AAB}$ & A & $\mathrm{BCC}$ & B & BBD & $\mathrm{BBC}$ & & $\mathrm{BCC}$ & B & BDD & 1156 \\
\hline 108 & 163 & $\mathrm{~F}$ & 160329 & BDD & $\mathrm{AAB}$ & $\mathrm{AAB}$ & $\mathrm{BCC}$ & B & BBD & & AAC & $\mathrm{BCC}$ & B & BDD & 921 \\
\hline 109 & 163 & $\mathrm{~F}$ & 162890 & BDD & $\mathrm{AAB}$ & $\mathrm{AAB}$ & $\mathrm{BCC}$ & B & BBD & $\mathrm{BBC}$ & AAC & $\mathrm{BBC}$ & B & BDD & 1232 \\
\hline 110) & 163 & $\mathrm{~F}$ & 162891 & BDD & $\mathrm{AAB}$ & $\mathrm{AAB}$ & $\mathrm{BCC}$ & B & BBD & $\mathrm{BBC}$ & AAC & $\mathrm{BBC}$ & B & BDD & 1223 \\
\hline 111 & 163 & $\mathrm{~F}$ & 162892 & BDD & $\mathrm{AAB}$ & $\mathrm{AAB}$ & $\mathrm{BCC}$ & B & BBD & $\mathrm{BBC}$ & $\mathrm{ABC}$ & $\mathrm{BBC}$ & B & BDD & 1017 \\
\hline 112 & 163 & F & 162893 & BDD & $\mathrm{AAB}$ & $\mathrm{AAB}$ & BCC & B & BBD & $\mathrm{BBC}$ & $\mathrm{ABC}$ & $\mathrm{BBC}$ & B & BDD & 1084 \\
\hline 113 & 163 & $\mathrm{~F}$ & 162894 & BDD & $\mathrm{AAB}$ & $\mathrm{AAB}$ & $\mathrm{BCC}$ & B & BBD & $\mathrm{BBC}$ & AAC & B & B & BDD & 1128 \\
\hline 114 & 163 & $\mathrm{~F}$ & 162895 & BDD & $\mathrm{AAB}$ & $\mathrm{AAB}$ & $\mathrm{BCC}$ & B & BBD & $\mathrm{BBC}$ & AAC & $\mathrm{BBC}$ & B & BDD & 1056 \\
\hline 115 & 163 & F & 162896 & BDD & $\mathrm{AAB}$ & $\mathrm{AAB}$ & $\mathrm{BCC}$ & B & BBD & $\mathrm{BBC}$ & AAC & $\mathrm{BBC}$ & B & BDD & 1064 \\
\hline 116 & 163 & $\mathrm{~F}$ & 162902 & BDD & $\mathrm{AAB}$ & $\mathrm{AAB}$ & $\mathrm{BCC}$ & B & BBD & $\mathrm{BBC}$ & - & $\mathrm{BBC}$ & B & BDD & 1094 \\
\hline 117 & 164 & $\mathrm{~F}$ & 162886 & BDD & $\mathrm{AAB}$ & $\mathrm{AAB}$ & $\mathrm{BCC}$ & B & BBD & $\mathrm{BBC}$ & AAC & $\mathrm{BBC}$ & B & BDD & 963 \\
\hline 118 & 164 & $\mathrm{~F}$ & 162897 & BDD & $\mathrm{AAB}$ & $\mathrm{AAB}$ & $\mathrm{BCC}$ & B & BBD & $\mathrm{BBC}$ & - & $\mathrm{BBC}$ & B & BDD & 1045 \\
\hline 119 & 164 & F & 162898 & BDD & $\mathrm{AAB}$ & $\mathrm{AAB}$ & $\mathrm{BCC}$ & B & BBD & $\mathrm{BBC}$ & - & $\mathrm{BCC}$ & B & BDD & 1070 \\
\hline 120) & 164 & $\mathrm{~F}$ & 162899 & BDD & $\mathrm{AAB}$ & $\mathrm{AAB}$ & $\mathrm{BCC}$ & B & BBD & $\mathrm{BBC}$ & $\mathrm{ABC}$ & $\mathrm{BCC}$ & B & BDD & 1173 \\
\hline 121 & 164 & $\mathrm{~F}$ & 162901 & BDD & $\mathrm{AAB}$ & $\mathrm{AAB}$ & $\mathrm{BCC}$ & B & BBD & $\mathrm{BBC}$ & - & $\mathrm{BCC}$ & B & BDD & 1097 \\
\hline 122 & 164 & $\mathrm{~F}$ & 163554 & BDD & $\mathrm{AAB}$ & $\mathrm{AAB}$ & $\mathrm{BCC}$ & B & BBD & $\mathrm{BBC}$ & $\mathrm{ABC}$ & $\mathrm{BCC}$ & $\mathrm{B}$ & BDD & 1080 \\
\hline 123 & 165 & $\mathrm{~F}$ & 160321 & BDD & $\mathrm{AAB}$ & $\mathrm{ABB}$ & $\mathrm{BBC}$ & B & BBD & - & - & $\mathrm{BCC}$ & B & BDD & - \\
\hline 124 & 165 & $\mathrm{~F}$ & 160325 & BDD & $\mathrm{AAB}$ & $\mathrm{AAB}$ & $\mathrm{BCC}$ & B & BBD & - & AAC & $\mathrm{BBC}$ & B & BDD & 1113 \\
\hline 125 & 165 & F & 160326 & BDD & $\mathrm{AAB}$ & $\mathrm{AAB}$ & BCC & B & BBD & - & AAC & $\mathrm{BBC}$ & B & BDD & 1258 \\
\hline 126 & 165 & $\mathrm{~F}$ & 160327 & BDD & $\mathrm{AAB}$ & $\mathrm{AAB}$ & $\mathrm{BBC}$ & B & BBD & - & AAC & $\mathrm{BBC}$ & B & BDD & 950 \\
\hline 127 & 165 & $\mathrm{~F}$ & 160328 & BDD & $\mathrm{AAB}$ & $\mathrm{AAB}$ & $\mathrm{BCC}$ & B & BBD & - & AAC & B & B & BDD & 1347 \\
\hline 128 & 165 & F & 162910 & BDD & $\mathrm{AAB}$ & $\mathrm{AAB}$ & BCC & B & BBD & $\mathrm{BBC}$ & $\mathrm{ABC}$ & BCC & B & BDD & 1087 \\
\hline 129 & 165 & F & 162911 & BDD & $\mathrm{AAB}$ & $\mathrm{AAB}$ & $\mathrm{BCC}$ & B & BBD & $\mathrm{BBC}$ & AAC & $\mathrm{BBC}$ & B & BDD & 1065 \\
\hline 130) & 165 & $\mathrm{~F}$ & 162912 & BDD & $\mathrm{AAB}$ & $\mathrm{AAB}$ & $\mathrm{BCC}$ & B & BBD & $\mathrm{BBC}$ & AAC & B & B & BDD & 1094 \\
\hline 131 & 165 & F & 162913 & BDD & $\mathrm{ABB}$ & $\mathrm{AAB}$ & $\mathrm{BCC}$ & B & BBD & $\mathrm{BBC}$ & $\mathrm{ABC}$ & $\mathrm{BBC}$ & B & BDD & 1036 \\
\hline 132 & 165 & F & 162914 & BDD & $\mathrm{AAB}$ & $\mathrm{AAB}$ & $\mathrm{BCC}$ & B & BBD & $\mathrm{BBC}$ & $\mathrm{ABC}$ & $\mathrm{BBC}$ & B & BDD & 1021 \\
\hline 133 & 165 & $\mathrm{~F}$ & 162915 & BDD & $\mathrm{ABB}$ & $\mathrm{AAB}$ & $\mathrm{BCC}$ & B & BBD & $\mathrm{BBC}$ & $\mathrm{ABC}$ & $\mathrm{BBC}$ & B & BDD & 1069 \\
\hline 134 & 165 & F & 162916 & BDD & $\mathrm{AAB}$ & $\mathrm{AAB}$ & $\mathrm{BCC}$ & B & BBD & $\mathrm{BBC}$ & AAC & B & B & BDD & 1132 \\
\hline 135 & 165 & F & 162917 & BDD & $\mathrm{AAB}$ & $\mathrm{AAB}$ & $\mathrm{BCC}$ & B & BBD & $\mathrm{BBC}$ & AAC & B & B & BDD & 1108 \\
\hline 136 & 165 & F & 162918 & BDD & $\mathrm{AAB}$ & $\mathrm{AAB}$ & $\mathrm{BCC}$ & B & BBD & $\mathrm{BBC}$ & AAC & $\mathrm{BCC}$ & B & BDD & 1202 \\
\hline 137 & 165 & M & 162919 & BDD & $\mathrm{AAB}$ & $\mathrm{AAB}$ & $\mathrm{BCC}$ & B & BBD & $\mathrm{BBC}$ & AAC & BCC & B & BDD & 1142 \\
\hline 138 & 165 & F & 162920 & BDD & $\mathrm{AAB}$ & $\mathrm{AAB}$ & BCC & B & BBD & $\mathrm{BBC}$ & AAC & $\mathrm{BBC}$ & B & BDD & 1204 \\
\hline 139 & 165 & $\mathrm{~F}$ & 162921 & BDD & $\mathrm{AAB}$ & $\mathrm{AAB}$ & $\mathrm{BCC}$ & B & BBD & $\mathrm{BBC}$ & $\mathrm{ABC}$ & $\mathrm{BBC}$ & B & BDD & 1064 \\
\hline 140) & 166 & $\mathrm{~F}$ & 162881 & BDD & $\mathrm{AAB}$ & $\mathrm{AAB}$ & $\mathrm{BCC}$ & B & BBD & $\mathrm{BBC}$ & $\mathrm{ABC}$ & $\mathrm{BBC}$ & B & BDD & 1103 \\
\hline 141 & 166 & F & 162882 & BDD & $\mathrm{AAB}$ & $\mathrm{AAB}$ & $\mathrm{BCC}$ & B & BBD & $\mathrm{BBC}$ & AAC & $\mathrm{BBC}$ & B & BDD & 1091 \\
\hline 142 & 166 & $\mathrm{~F}$ & 162883 & BDD & $\mathrm{AAB}$ & $\mathrm{AAB}$ & $\mathrm{BCC}$ & B & BBD & $\mathrm{BBC}$ & AAC & $\mathrm{BBC}$ & B & BDD & 1111 \\
\hline 143 & 166 & $\mathrm{~F}$ & 162884 & BDD & $\mathrm{AAB}$ & $\mathrm{AAB}$ & $\mathrm{BCC}$ & B & BBD & $\mathrm{BBC}$ & $\mathrm{ABC}$ & $\mathrm{BCC}$ & B & BDD & 1182 \\
\hline 144 & 166 & F & 162885 & BDD & $\mathrm{AAB}$ & $\mathrm{AAB}$ & $\mathrm{BCC}$ & B & BBD & $\mathrm{BBC}$ & $\mathrm{ABC}$ & $\mathrm{BBC}$ & B & BDD & 1195 \\
\hline 145 & 166 & F & 162903 & BBD & $\mathrm{AAB}$ & $\mathrm{ABB}$ & $\mathrm{BCC}$ & B & BBD & $\mathrm{BBC}$ & $\mathrm{ABC}$ & $\mathrm{BBC}$ & B & BDD & 1148 \\
\hline 146 & 166 & F & 162904 & BDD & $\mathrm{AAB}$ & $\mathrm{AAB}$ & $\mathrm{BCC}$ & B & BBD & - & $\mathrm{ABC}$ & $\mathrm{BCC}$ & B & BDD & 1132 \\
\hline 147 & 166 & F & 162905 & BDD & $\mathrm{AAB}$ & $\mathrm{AAB}$ & $\mathrm{BCC}$ & B & BBD & & $\mathrm{ABC}$ & $\mathrm{BBC}$ & B & BDD & 911 \\
\hline 148 & 166 & F & 162906 & BDD & $\mathrm{AAB}$ & $\mathrm{AAB}$ & $\mathrm{BCC}$ & B & BBD & $\mathrm{BBC}$ & $\mathrm{ABC}$ & $\mathrm{BCC}$ & B & BDD & 1106 \\
\hline 149 & 166 & F & 162907 & BDD & $\mathrm{AAB}$ & $\mathrm{AAB}$ & BCC & B & BBD & - & AAC & $\mathrm{BBC}$ & B & BDD & 1030 \\
\hline 150) & 166 & F & 162908 & BDD & $\mathrm{AAB}$ & $\mathrm{AAB}$ & $\mathrm{BCC}$ & B & BBD & 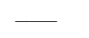 & $\mathrm{ABC}$ & $\mathrm{BBC}$ & B & BDD & 989 \\
\hline 151 & 166 & F & 162909 & BDD & $\mathrm{AAB}$ & $\mathrm{AAB}$ & BCC & B & BBD & - & $\mathrm{ABC}$ & $\mathrm{BBC}$ & B & BDD & 1113 \\
\hline 152 & 167 & $\mathrm{~F}$ & 162887 & BDD & $\mathrm{AAB}$ & $\mathrm{AAB}$ & $\mathrm{BCC}$ & B & BBD & $\mathrm{BCC}$ & AAC & $\mathrm{BBC}$ & B & BDD & 1209 \\
\hline 153 & 167 & F & 162888 & BDD & $\mathrm{AAB}$ & $\mathrm{AAB}$ & BCC & B & BBD & $\mathrm{BCC}$ & AAC & $\mathrm{BBC}$ & B & BDD & 1022 \\
\hline 154 & 167 & F & 162889 & BDD & $\mathrm{AAB}$ & $\mathrm{AAB}$ & $\mathrm{BCC}$ & B & BBD & $\mathrm{BCC}$ & AAC & $\mathrm{BBC}$ & B & BDD & 1021 \\
\hline 155 & 169 & F & 167007 & BDD & $\mathrm{ABB}$ & $\mathrm{AAB}$ & $\mathrm{BCC}$ & B & BBD & $\mathrm{BBC}$ & & $\mathrm{BCC}$ & B & BDD & 1131 \\
\hline
\end{tabular}


APPENDIX 2-5

(Continued)

\begin{tabular}{|c|c|c|c|c|c|c|c|c|c|c|c|c|c|c|c|}
\hline & \multirow[b]{2}{*}{ Site } & \multirow[b]{2}{*}{ Sex } & \multirow[b]{2}{*}{ AMNH } & \multicolumn{12}{|c|}{ Locus } \\
\hline & & & & Aat-1 & Aat-2 & Idh-1 & Ldh-1 & Ldh-2 & Mdh-1 & Mpi & Pgi & Pgm-1 & Pgm-2 & Sod-1 & blood \\
\hline 156 & 169 & F & 167008 & BDD & AAB & $\mathrm{ABB}$ & $\mathrm{BCC}$ & B & BBD & $\mathrm{BCC}$ & - & BCC & B & BDD & 917 \\
\hline 157 & 169 & $\mathrm{~F}$ & 167009 & BDD & $\mathrm{AAB}$ & $\mathrm{AAB}$ & $\mathrm{BCC}$ & B & BBD & $\mathrm{BBC}$ & $\mathrm{BBC}$ & $\mathrm{C}$ & B & BDD & 1127 \\
\hline 158 & 169 & $\mathrm{~F}$ & 167010 & BDD & $\mathrm{AAB}$ & A & $\mathrm{BCC}$ & B & BBD & $\mathrm{BBC}$ & - & $\mathrm{BCC}$ & B & BDD & 1129 \\
\hline 159 & 169 & $\mathrm{~F}$ & 167011 & BDD & $\mathrm{AAB}$ & $\mathrm{AAB}$ & $\mathrm{BBC}$ & B & BBD & $\mathrm{BBC}$ & $\mathrm{BBC}$ & C & B & BDD & 1033 \\
\hline 160) & 179 & $\mathrm{~F}$ & 169826 & BDD & $\mathrm{AAB}$ & $\mathrm{AAB}$ & $\mathrm{BCC}$ & B & BBC & $\mathrm{ABC}$ & C & . & B & BDD & $\mathrm{FCM}$ \\
\hline 161 & 179 & $\mathrm{~F}$ & 169827 & BDD & $\mathrm{AAB}$ & $\mathrm{AAB}$ & BCC & B & BBD & $\mathrm{AAB}$ & . & $\mathrm{BCC}$ & B & BDD & FCM \\
\hline 162 & 179 & $\mathrm{~F}$ & 169828 & BDD & $\mathrm{AAB}$ & $\mathrm{AAB}$ & $\mathrm{BCC}$ & B & $\mathrm{BBC}$ & & 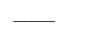 & BCC & B & BDD & $\mathrm{FCM}$ \\
\hline 163 & 179 & $\mathrm{~F}$ & 169829 & BDD & $\mathrm{AAB}$ & A & $\mathrm{BCC}$ & B & $\mathrm{BBC}$ & $\mathrm{ABC}$ & C & - & B & BDD & $\mathrm{FCM}$ \\
\hline 164 & 179 & $\mathrm{~F}$ & 169830 & BDD & $\mathrm{AAB}$ & $\mathrm{AAB}$ & $\mathrm{BCC}$ & B & $\mathrm{BBC}$ & $\mathrm{ABC}$ & $\mathrm{BCC}$ & - & B & BDD & $\mathrm{FCM}$ \\
\hline 165 & 179 & $\mathrm{~F}$ & 169831 & BDD & AAB & $\mathrm{AAB}$ & $\mathrm{BCC}$ & B & $\mathrm{BBC}$ & - & - & BCC & B & BDD & - \\
\hline 166 & 179 & $\mathrm{~F}$ & 169832 & BDD & $\mathrm{AAB}$ & $\mathrm{AAB}$ & $\mathrm{BCC}$ & B & $\mathrm{BBC}$ & - & - & $\mathrm{BCC}$ & B & BDD & . \\
\hline 167 & 191 & $\mathrm{~F}$ & 169839 & BDD & AAB & $\mathrm{AAB}$ & $\mathrm{BCC}$ & B & BBD & . & AAC & $\mathrm{BBC}$ & B & BDD & $\mathrm{FCM}$ \\
\hline 168 & 191 & $\mathrm{~F}$ & 162936 & BDD & $\mathrm{AAB}$ & $\mathrm{AAB}$ & BCC & B & BBD & $\mathrm{BBC}$ & - & - & B & BDD & 966 \\
\hline 169 & 191 & $\mathrm{~F}$ & 162937 & BDD & $\mathrm{AAB}$ & $\mathrm{AAB}$ & BCC & B & BBD & $\mathrm{BBC}$ & - & - & $\mathrm{BBC}$ & BDD & 1158 \\
\hline 170) & 191 & F & 162940 & BDD & $\mathrm{AAB}$ & $\mathrm{AAB}$ & BCC & B & BBD & $\mathrm{BBC}$ & AAC & B & - & BDD & 1104 \\
\hline 171 & 191 & $\mathrm{~F}$ & 162941 & BDD & $\mathrm{AAB}$ & $\mathrm{AAB}$ & BCC & B & BBD & $\mathrm{BBC}$ & AAC & BCC & B & BDD & 812 \\
\hline 172 & 191 & F & 163458 & BDD & $\mathrm{AAB}$ & $\mathrm{AAB}$ & $\mathrm{BCC}$ & B & BBD & $\mathrm{BBC}$ & - & B & B & BDD & 1009 \\
\hline 173 & 192 & $\mathrm{~F}$ & 169768 & BDD & $\mathrm{AAB}$ & $\mathrm{ABB}$ & $\mathrm{BCC}$ & B & BBD & $\mathrm{BBC}$ & AAC & $\mathrm{BBC}$ & B & BDD & $\mathrm{FCM}$ \\
\hline 174 & 193 & $\mathrm{~F}$ & 169769 & BDD & $\mathrm{AAB}$ & $\mathrm{AAB}$ & BCC & B & BBD & $\mathrm{BCC}$ & - & B & B & BDD & $\mathrm{FCM}$ \\
\hline 175 & 193 & $\mathrm{~F}$ & 169770 & BDD & $\mathrm{AAB}$ & $\mathrm{AAB}$ & $\mathrm{BCC}$ & B & BBD & $\mathrm{BCC}$ & - & B & B & BDD & $\mathrm{FCM}$ \\
\hline 176 & 193 & $\mathrm{~F}$ & 169771 & BDD & $\mathrm{AAB}$ & $\mathrm{AAB}$ & $\mathrm{BCC}$ & $\mathrm{BB}$ & BBD & $\mathrm{BBC}$ & - & B & B & BDD & $\mathrm{FCM}$ \\
\hline 177 & 194 & $\mathrm{~F}$ & 165972 & BDD & $\mathrm{AAB}$ & $\mathrm{AAB}$ & $\mathrm{BCC}$ & B & BBD & - & - & $\mathrm{BBC}$ & B & BDD & 1106 \\
\hline 178 & 194 & F & 165973 & BDD & $\mathrm{AAB}$ & $\mathrm{ABB}$ & $\mathrm{BCC}$ & B & BBD & - & - & $\mathrm{BBC}$ & B & BDD & 1031 \\
\hline 179 & 194 & $\mathrm{~F}$ & 165974 & BDD & $\mathrm{AAB}$ & $\mathrm{AAB}$ & $\mathrm{BCC}$ & B & BBD & - & - & $\mathrm{BBC}$ & B & BDD & 851 \\
\hline 180) & 194 & $\mathrm{~F}$ & 165975 & BDD & $\mathrm{AAB}$ & $\mathrm{AAB}$ & BCC & B & BBD & B & AAC & - & B & BDD & 1029 \\
\hline 181 & 194 & $\mathrm{~F}$ & 165976 & BDD & $\mathrm{AAB}$ & $\mathrm{ABB}$ & BCC & B & BBD & - & AAC & - & B & BDD & 1060 \\
\hline 182 & 194 & $\mathrm{~F}$ & 165977 & BDD & $\mathrm{AAB}$ & $\mathrm{AAB}$ & $\mathrm{BCC}$ & B & BBD & - & AAC & - & B & BDD & 1142 \\
\hline 183 & 194 & $\mathrm{~F}$ & 165978 & BDD & $\mathrm{AAB}$ & $\mathrm{AAB}$ & BCC & B & BBD & - & AAC & $\mathrm{BBC}$ & B & BDD & 1211 \\
\hline 184 & 195 & $\mathrm{~F}$ & 158788 & BDD & $\mathrm{AAB}$ & A & $\mathrm{BCC}$ & B & BBD & $\mathrm{BBC}$ & AAC & B & B & BDD & 1022 \\
\hline 185 & 195 & $\mathrm{~F}$ & 158789 & BDD & $\mathrm{AAB}$ & $\mathrm{AAB}$ & $\mathrm{BCC}$ & B & BBD & $\mathrm{BBC}$ & - & B & B & BDD & 1031 \\
\hline 186 & 195 & $\mathrm{~F}$ & 158790 & BDD & $\mathrm{AAB}$ & $\mathrm{AAB}$ & $\mathrm{BCC}$ & B & BBD & $\mathrm{BBC}$ & - & B & B & BDD & 1000 \\
\hline 187 & 195 & $\mathrm{~F}$ & 158791 & BDD & $\mathrm{AAB}$ & $\mathrm{AAB}$ & $\mathrm{BCC}$ & B & BBD & $\mathrm{BBC}$ & - & B & B & BDD & 1056 \\
\hline 188 & 195 & F & 158792 & BDD & $\mathrm{AAB}$ & $\mathrm{AAB}$ & $\mathrm{BCC}$ & B & BBD & $\mathrm{BBC}$ & - & B & B & BDD & 1010 \\
\hline 189 & 195 & F & 158793 & BDD & $\mathrm{ABB}$ & $\mathrm{AAB}$ & $\mathrm{BCC}$ & B & BBD & $\mathrm{BBC}$ & - & B & B & BDD & 1033 \\
\hline 190) & 195 & $\mathrm{~F}$ & 158794 & BDD & $\mathrm{AAB}$ & $\mathrm{AAB}$ & $\mathrm{BCC}$ & B & BBD & $\mathrm{BBC}$ & - & B & B & BDD & 966 \\
\hline 191 & 195 & $\mathrm{~F}$ & 158795 & BDD & $\mathrm{AAB}$ & $\mathrm{AAB}$ & $\mathrm{BCC}$ & B & BBD & $\mathrm{BBC}$ & - & B & B & BDD & 978 \\
\hline 192 & 195 & $\mathrm{~F}$ & 158796 & BDD & $\mathrm{AAB}$ & $\mathrm{AAB}$ & BCC & B & BBD & $\mathrm{BBC}$ & - & B & B & BDD & 1003 \\
\hline 193 & 198 & $\mathrm{~F}$ & 158823 & BDD & $\mathrm{AAB}$ & $\mathrm{AAB}$ & $\mathrm{BCC}$ & B & BBD & $\mathrm{BBC}$ & - & B & B & BDD & 770 \\
\hline 194 & 198 & $\mathrm{~F}$ & 169349 & BDD & $\mathrm{AAB}$ & $\mathrm{AAB}$ & $\mathrm{BCC}$ & B & BBD & $\mathrm{BBC}$ & - & B & B & BDD & FCM \\
\hline 195 & 198 & $\mathrm{~F}$ & 169350 & BDD & $\mathrm{AAB}$ & - & BCC & B & BBD & $\mathrm{BBC}$ & - & B & B & BDD & FCM \\
\hline 196 & 198 & $\mathrm{~F}$ & 169351 & BDD & $\mathrm{AAB}$ & - & BCC & $\mathrm{AB}$ & BBD & $\mathrm{BBC}$ & - & B & B & BDD & FCM \\
\hline 197 & 200 & $\mathrm{~F}$ & 169840 & BDD & $\mathrm{AAB}$ & $\mathrm{AAB}$ & $\mathrm{BCC}$ & B & BBD & $\mathrm{BBC}$ & AAC & B & B & BDD & FCM \\
\hline 198 & 200 & F & 169841 & BDD & $\mathrm{AAB}$ & $\mathrm{AAB}$ & BCC & B & BBD & $\mathrm{BBC}$ & AAC & B & B & BDD & FCM \\
\hline 199 & 200 & $\mathrm{~F}$ & 169842 & BDD & $\mathrm{AAB}$ & $\mathrm{AAB}$ & $\mathrm{BCC}$ & B & BBD & $\mathrm{BBC}$ & AAC & B & B & BDD & FCM \\
\hline 200) & 200 & $\mathrm{~F}$ & 169843 & BDD & $\mathrm{AAB}$ & $\mathrm{ABB}$ & $\mathrm{BCC}$ & B & BBD & $\mathrm{BBC}$ & AAC & B & B & BDD & $\mathrm{FCM}$ \\
\hline 201 & 200 & F & 169844 & BDD & $\mathrm{AAB}$ & $\mathrm{AAB}$ & $\mathrm{BCC}$ & B & BBD & $\mathrm{BBC}$ & AAC & B & B & BDD & FCM \\
\hline 202 & 200 & F & 169845 & BDD & $\mathrm{AAB}$ & $\mathrm{AAB}$ & BCC & B & BBD & $\mathrm{BBC}$ & AAC & B & B & BDD & $\mathrm{FCM}$ \\
\hline 203 & 200 & $\mathrm{~F}$ & 169846 & BDD & $\mathrm{AAB}$ & $\mathrm{AAB}$ & $\mathrm{BCC}$ & B & BBD & $\mathrm{BBC}$ & AAC & B & B & BDD & FCM \\
\hline 204 & 200 & $\mathrm{~F}$ & 169847 & BDD & $\mathrm{AAB}$ & $\mathrm{AAB}$ & $\mathrm{BCC}$ & B & BBD & $\mathrm{BBC}$ & A & B & B & BDD & FCM \\
\hline 205 & 200 & $\mathrm{~F}$ & 169848 & BDD & - & $\mathrm{AAB}$ & $\mathrm{BCC}$ & B & BBD & - & AAC & B & B & BDD & - \\
\hline 206 & 202 & $\mathrm{~F}$ & 165979 & BDD & $\mathrm{AAB}$ & $\mathrm{AAB}$ & $\mathrm{BCC}$ & B & BBD & $\mathrm{BBC}$ & - & B & B & BDD & 932 \\
\hline 207 & 202 & $\mathrm{~F}$ & 165980 & BDD & $\mathrm{AAB}$ & $\mathrm{AAB}$ & BCC & B & BBD & $\mathrm{BBC}$ & - & B & B & BDD & 1067 \\
\hline
\end{tabular}


APPENDIX 2-5

(Continued)

\begin{tabular}{|c|c|c|c|c|c|c|c|c|c|c|c|c|c|c|c|}
\hline & \multirow[b]{2}{*}{ Site } & \multirow[b]{2}{*}{ Sex } & \multirow[b]{2}{*}{ AMNH } & \multicolumn{12}{|c|}{ Locus } \\
\hline & & & & Aat-1 & Aat-2 & Idh-1 & Ldh-1 & Ldh-2 & Mdh-1 & Mpi & Pgi & Pgm-1 & Pgm-2 & Sod-1 & blood \\
\hline 208 & 202 & $\mathrm{~F}$ & 165981 & BDD & $\mathrm{AAB}$ & AAB & $\mathrm{BCC}$ & B & $\mathrm{BBD}$ & $\mathrm{BBC}$ & 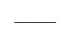 & B & B & BDD & 1036 \\
\hline 209 & 203 & $\mathrm{~F}$ & 165982 & BDD & $\mathrm{AAB}$ & AAB & $\mathrm{BCC}$ & B & BBD & $\mathrm{BBC}$ & - & B & B & BDD & 1029 \\
\hline 210) & 203 & $\mathrm{~F}$ & 165983 & BDD & $\mathrm{AAB}$ & $\mathrm{AAB}$ & $\mathrm{BCC}$ & B & $\mathrm{BBD}$ & $\mathrm{BBC}$ & 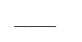 & B & B & BDD & 1081 \\
\hline 211 & 203 & $\mathrm{~F}$ & 165984 & BDD & $\mathrm{AAB}$ & $\mathrm{AAB}$ & $\mathrm{BCC}$ & B & BBD & $\mathrm{BBC}$ & - & B & B & BDD & 1127 \\
\hline 212 & 203 & $\mathrm{~F}$ & 165985 & BDD & $\mathrm{AAB}$ & $\mathrm{AAB}$ & $\mathrm{BCC}$ & B & $\mathrm{BBD}$ & - & $\mathrm{AAC}$ & $\mathrm{BBC}$ & B & BDD & 1125 \\
\hline 213 & 203 & $\mathrm{~F}$ & 165986 & BDD & AAB & AAB & $\mathrm{BCC}$ & B & BBD & $\mathrm{BBC}$ & - & B & B & BDD & 1076 \\
\hline 214 & 203 & $\mathrm{~F}$ & 165988 & BDD & $\mathrm{AAB}$ & $\mathrm{AAB}$ & $\mathrm{BCC}$ & B & $\mathrm{BBD}$ & $\mathrm{BBC}$ & - & $\mathrm{BBC}$ & B & BDD & 991 \\
\hline 215 & 203 & $\mathrm{~F}$ & 165989 & BDD & $\mathrm{AAB}$ & $\mathrm{ABB}$ & $\mathrm{BBC}$ & B & $\mathrm{BBD}$ & $\mathrm{BBC}$ & - & B & B & BDD & 1165 \\
\hline 216 & 203 & $\mathrm{~F}$ & 165990 & BDD & $\mathrm{AAB}$ & AAB & $\mathrm{BCC}$ & B & BBD & $\mathrm{BBC}$ & 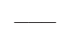 & $\mathrm{BBC}$ & B & BDD & 1070 \\
\hline 217 & 203 & $\mathrm{~F}$ & 165991 & BDD & $\mathrm{AAB}$ & $\mathrm{AAB}$ & $\mathrm{BCC}$ & B & BBD & $\mathrm{BBC}$ & - & $\mathrm{BCC}$ & B & BDD & 974 \\
\hline 218 & 203 & $\mathrm{~F}$ & 165992 & BDD & $\mathrm{AAB}$ & $\mathrm{AAB}$ & $\mathrm{BCC}$ & B & $\mathrm{BBD}$ & - & $\mathrm{AAC}$ & $\mathrm{B}$ & B & BDD & 1123 \\
\hline 219 & 203 & $\mathrm{~F}$ & 165993 & BDD & $\mathrm{AAB}$ & $\mathrm{AAB}$ & $\mathrm{BCC}$ & B & $\mathrm{BBD}$ & - & $\mathrm{AAC}$ & B & B & BDD & 1182 \\
\hline 220) & 207 & $\mathrm{~F}$ & 169870 & BDD & $\mathrm{AAB}$ & $\mathrm{AAB}$ & $\mathrm{BCC}$ & B & BBD & - & AAC & B & B & BDD & FCM \\
\hline 221 & 207 & $\mathrm{~F}$ & 169871 & BDD & $\mathrm{AAB}$ & $\mathrm{AAB}$ & $\mathrm{BCC}$ & B & BBD & - & $\mathrm{AAC}$ & B & B & BDD & FCM \\
\hline 222 & 207 & $\mathrm{~F}$ & 169872 & BDD & $\mathrm{AAB}$ & $\mathrm{AAB}$ & $\mathrm{BCC}$ & ABB & BBD & - & AAC & B & B & BDD & FCM \\
\hline 223 & 207 & $\mathrm{~F}$ & 169873 & BDD & $\mathrm{AAB}$ & $\mathrm{AAB}$ & $\mathrm{BCC}$ & B & BBD & - & AAC & B & B & BDD & FCM \\
\hline 224 & 207 & $\mathrm{~F}$ & 169874 & BDD & $\mathrm{AAB}$ & AAB & $\mathrm{BCC}$ & ABB & $\mathrm{BBD}$ & - & AAC & B & B & BDD & FCM \\
\hline 225 & 207 & $\mathrm{~F}$ & 169875 & BDD & $\mathrm{AAB}$ & $\mathrm{AAB}$ & $\mathrm{BCC}$ & B & BBD & - & AAC & B & B & BDD & $\mathrm{FCM}$ \\
\hline 226 & 207 & $\mathrm{~F}$ & 169876 & BDD & $\mathrm{AAB}$ & $\mathrm{AAB}$ & $\mathrm{BCC}$ & B & BBD & - & AAC & B & B & BDD & $\mathrm{FCM}$ \\
\hline 227 & 207 & $\mathrm{~F}$ & 169877 & BDD & $\mathrm{AAB}$ & $\mathrm{AAB}$ & $\mathrm{BCC}$ & B & BBD & - & $\mathrm{AAC}$ & B & B & BDD & FCM \\
\hline 228 & 207 & $\mathrm{~F}$ & 169878 & BDD & $\mathrm{AAB}$ & $\mathrm{AAB}$ & $\mathrm{BCC}$ & ABB & $\mathrm{BBD}$ & - & $\mathrm{AAC}$ & B & B & BDD & FCM \\
\hline 229 & 207 & $\mathrm{~F}$ & 169879 & BDD & $\mathrm{AAB}$ & $\mathrm{ABB}$ & $\mathrm{BCC}$ & B & BBD & - & AAC & B & B & BDD & FCM \\
\hline 230) & 208 & $\mathrm{~F}$ & 158797 & BDD & $\mathrm{AAB}$ & $\mathrm{ABB}$ & $\mathrm{BCC}$ & B & BBD & $\mathrm{BBC}$ & AAC & B & B & BDD & 1356 \\
\hline 231 & 208 & $\mathrm{~F}$ & 158798 & BDD & $\mathrm{AAB}$ & $\mathrm{AAB}$ & $\mathrm{BCC}$ & B & BBD & $\mathrm{BBC}$ & - & B & B & BDD & 1181 \\
\hline 232 & 208 & $\mathrm{~F}$ & 158799 & BDD & $\mathrm{AAB}$ & $\mathrm{AAB}$ & $\mathrm{BCC}$ & B & $\mathrm{BBD}$ & $\mathrm{BBC}$ & - & B & B & BDD & 1140 \\
\hline 233 & 208 & $\mathrm{~F}$ & 158800 & BDD & $\mathrm{AAB}$ & A & $\mathrm{BBC}$ & B & BBD & $\mathrm{BBC}$ & $\mathrm{ABC}$ & B & B & BDD & 1144 \\
\hline 234 & 207 & $\mathrm{~F}$ & 158801 & BDD & $\mathrm{AAB}$ & $\mathrm{AAB}$ & $\mathrm{BCC}$ & B & BBD & $\mathrm{BBC}$ & - & B & B & BDD & 1205 \\
\hline 235 & 208 & $\mathrm{~F}$ & 158802 & BDD & $\mathrm{AAB}$ & $\mathrm{A}$ & $\mathrm{BCC}$ & B & $\mathrm{BBD}$ & $\mathrm{BBC}$ & $\mathrm{AAC}$ & B & B & BDD & 1065 \\
\hline 236 & 208 & $\mathrm{~F}$ & 158803 & BDD & $\mathrm{AAB}$ & $\mathrm{AAB}$ & $\mathrm{BCC}$ & B & BBD & $\mathrm{BBC}$ & - & B & B & BDD & 1146 \\
\hline 237 & 208 & $\mathrm{~F}$ & 158804 & BDD & $\mathrm{AAB}$ & $\mathrm{AAB}$ & $\mathrm{BCC}$ & B & $\mathrm{BBD}$ & $\mathrm{BBC}$ & - & B & B & BDD & 1144 \\
\hline 238 & 208 & $\mathrm{~F}$ & 158805 & BDD & $\mathrm{ABB}$ & $\mathrm{AAB}$ & $\mathrm{BCC}$ & B & BBD & $\mathrm{BBC}$ & - & B & B & BDD & 1243 \\
\hline 239 & 208 & $\mathrm{~F}$ & 158806 & BDD & $\mathrm{AAB}$ & $\mathrm{AAB}$ & $\mathrm{BCC}$ & B & BBD & $\mathrm{BBC}$ & - & B & B & BDD & 1077 \\
\hline 240) & 208 & $\mathrm{~F}$ & 158807 & BDD & $\mathrm{AAB}$ & $\mathrm{AAB}$ & $\mathrm{BCC}$ & B & BBD & $\mathrm{BBC}$ & $\mathrm{ABC}$ & B & B & BDD & 1127 \\
\hline 241 & 208 & $\mathrm{~F}$ & 158808 & BDD & $\mathrm{AAB}$ & $\mathrm{AAB}$ & $\mathrm{BCC}$ & B & $\mathrm{BBD}$ & $\mathrm{BBC}$ & - & B & B & BDD & 1168 \\
\hline 242 & 208 & $\mathrm{~F}$ & 158809 & BDD & $\mathrm{AAB}$ & $\mathrm{AAB}$ & $\mathrm{BCC}$ & B & BBD & $\mathrm{BBC}$ & - & B & B & BDD & 1116 \\
\hline 243 & 208 & $\mathrm{~F}$ & 158810 & BDD & $\mathrm{AAB}$ & A & $\mathrm{BCC}$ & B & $\mathrm{BBD}$ & $\mathrm{BBC}$ & $\mathrm{ABC}$ & B & B & BDD & 1089 \\
\hline 244 & 213 & $\mathrm{~F}$ & 153117 & BDD & $\mathrm{AAB}$ & $\mathrm{AAB}$ & $\mathrm{BCC}$ & B & $\mathrm{BBD}$ & - & - & B & B & BDD & 1020 \\
\hline
\end{tabular}


Appendix 2-6: Genotypes of tetraploid (4n) Ambystoma (3) laterale - jeffersonianum (LLLJ) unisexual specimens ordered by site number.

\begin{tabular}{|c|c|c|c|c|c|c|c|c|c|c|c|c|c|c|c|}
\hline & \multirow[b]{2}{*}{ Site } & \multirow[b]{2}{*}{ Sex } & \multirow[b]{2}{*}{$\mathrm{AMNH}$} & \multicolumn{12}{|c|}{ Locus } \\
\hline & & & & Aat-1 & Aat-2 & Idh-1 & Ldh-1 & Ldh-2 & Mdh-1 & Mpi & Pgi & Pgm-1 & Pgm-2 & Sod-1 & blood \\
\hline 1 & 7 & $\mathrm{~F}$ & 158825 & BBBD & ABBB & ABBB & BBBC & B & BDDD & - & & B & B & BBBD & 1245 \\
\hline 2 & 7 & $\mathrm{~F}$ & 160339 & BBBD & $\mathrm{ABBB}$ & $\mathrm{ABBB}$ & BBBC & B & BDDD & $\mathrm{BCCC}$ & $\mathrm{BBCC}$ & B & $\mathrm{B}$ & BBBD & 1222 \\
\hline 3 & 7 & $\mathrm{~F}$ & 160340 & BBBD & ABBB & ABBB & BBBC & B & BDDD & BCCC & $\mathrm{BBCC}$ & B & B & BBBD & 1200 \\
\hline 4 & 107 & F & 169899 & BBBD & $\mathrm{ABBB}$ & $\mathrm{ABBB}$ & BBBC & A & BDDD & - & BCCC & B & 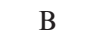 & BBBD & FCM \\
\hline 5 & 107 & F & 169909 & BBBD & $\mathrm{ABBB}$ & $\mathrm{ABBB}$ & BBBC & B & BDDD & - & $\mathrm{ABBB}$ & B & B & BBBD & FCM \\
\hline 6 & 107 & $\mathrm{~F}$ & 169910 & BBBD & $\mathrm{ABBB}$ & $\mathrm{ABBB}$ & BBBC & B & BDDD & & $\mathrm{BCCC}$ & B & $P$ & BBBD & FCM \\
\hline 7 & 107 & F & 169911 & BBBD & $\mathrm{ABBB}$ & $\mathrm{ABBB}$ & BBBC & B & BDDD & - & BCCC & B & B & BBBD & FCM \\
\hline 8 & 107 & F & 169912 & BBBD & $\mathrm{ABBB}$ & $\mathrm{ABBB}$ & BBBC & B & BDDD & - & $\mathrm{ABCC}$ & B & B & BBBD & FCM \\
\hline , & 108 & F & 160341 & BBBD & ABBB & ABBB & BBBC & B & BDDD & BCCC & $\mathrm{BCCC}$ & BBBC & B & BBBD & 1212 \\
\hline 10) & 108 & $\mathrm{~F}$ & 160342 & BBBD & - & $\mathrm{ABBB}$ & BBBC & B & BDDD & & - & $\mathrm{BBBC}$ & B & BBBD & 1229 \\
\hline 11 & 108 & F & 160343 & BBBD & ABBB & ABBB & BBBC & B & BDDD & B & - & BBBC & B & BBBD & 1286 \\
\hline 12 & 110 & $\mathrm{~F}$ & 169907 & BBBD & ABBB & ABBB & BBBC & B & BDDD & BCCC & ACCC & B & B & BBBD & FCM \\
\hline 13 & 110 & $\mathrm{~F}$ & 169908 & BBBD & $\mathrm{ABBB}$ & B & B & B & BBDD & 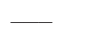 & A & B & B & BBD & $\mathrm{FCl}$ \\
\hline 14 & 111 & $\mathrm{~F}$ & 169411 & BBBD & ABBB & B & BBBC & B & BDDD & BCCC & $\mathrm{ABCC}$ & B & B & BBBD & FC \\
\hline 15 & 111 & F & 169412 & BBBD & $\mathrm{ABBB}$ & $\mathrm{ABBB}$ & BBBC & BBBC & BDDD & B & ACCC & B & B & B & FCI \\
\hline 16 & 111 & $\mathrm{~F}$ & 169413 & BBBD & $\mathrm{ABBB}$ & $\mathrm{ABBB}$ & BBBC & B & BDDD & $\mathrm{BCCC}$ & $\mathrm{ACCC}$ & B & B & BBBD & $\mathrm{FCN}$ \\
\hline 17 & 113 & $\mathrm{~F}$ & 162950 & BBBD & $\mathrm{ABBB}$ & $\mathrm{ABBB}$ & BBBC & B & BDDD & $\mathrm{BCCC}$ & $\mathrm{AACC}$ & B & B & BBBD & 1317 \\
\hline 18 & 148 & M & 167020 & BBBD & $\mathrm{ABBB}$ & $\mathrm{ABBB}$ & BBBC & B & BDDD & $\mathrm{BC}$ & $\mathrm{BC}$ & B & D & BBBD & 1190 \\
\hline 19 & 155 & $\mathrm{~F}$ & 160338 & BBBD & $\mathrm{ABBB}$ & $\mathrm{ABBB}$ & BBBC & B & BDDD & $\mathrm{BCCC}$ & $\mathrm{ACCC}$ & B & B & BBBD & 1251 \\
\hline 20) & 162 & $\mathrm{~F}$ & 167004 & BBDD & $\mathrm{ABBB}$ & $\mathrm{AABB}$ & $\mathrm{BBCC}$ & B & BDDD & BCCC & - & - & B & B & 1133 \\
\hline 21 & 168 & F & 160310 & BBBD & ABBB & ABBB & BBBC & ABBB & BDDD & BCCC & E- & $\mathrm{BBBC}$ & B & BBBD & 1290 \\
\hline 22 & 168 & F & 160318 & BBBD & $\mathrm{ABBB}$ & $\mathrm{ABBB}$ & BBBC & $\mathrm{ABBB}$ & BDDD & - & ACCC & $\mathrm{BBBC}$ & B & BBBD & 1030 \\
\hline 23 & 168 & $\mathrm{~F}$ & 160319 & BBBD & ABBB & ABBB & BBBC & AABB & BDDD & - & ACCC & $\mathrm{BBBC}$ & B & BBBD & 1113 \\
\hline 24 & 198 & $\mathrm{~F}$ & 169346 & BBBD & $\mathrm{ABBB}$ & $\mathrm{ABBB}$ & BBBC & B & BDDD & BCCC & E- & B & 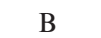 & BBBD & FCM \\
\hline 25 & 198 & F & 169347 & BBBD & ABBB & ABBB & BBBC & & BDDD & BCCC & $\mathrm{ACCC}$ & B & B & BBBD & FCM \\
\hline 26 & 198 & $\mathrm{~F}$ & 169348 & BBBD & $\mathrm{ABBB}$ & ABBB & BBBC & B & BDDD & BCCC & ACCC & B & B & BBBD & FCM \\
\hline
\end{tabular}


Appendix 2-7: Genotypes of tetraploid (4n) Ambystoma laterale - (3) jeffersonianum (LJJJ) unisexual specimens ordered by site number.

\begin{tabular}{|c|c|c|c|c|c|c|c|c|c|c|c|c|c|c|c|}
\hline & \multirow[b]{2}{*}{ Site } & \multirow[b]{2}{*}{ Sex } & \multirow[b]{2}{*}{$\mathrm{AMNH}$} & \multicolumn{12}{|c|}{ Locus } \\
\hline & & & & I Aat-1 & Aat-2 & Idh-1 & Ldh-1 & Ldh-2 & Mdh-1 & Mpi & Pgi & Pgm-1 & Pgm-2 & 2 Sod-1 & blood \\
\hline 1 & 109 & $\mathrm{~F}$ & 169922 & BDDD & AAAB & AAAB & BCCC & B & BBBD & - & $\mathrm{ABCC}$ & BCCC & B & BDDD & FCM \\
\hline 2 & 109 & $\mathrm{~F}$ & 169923 & BDDD & AAAB & AAAB & BCCC & $\mathrm{ABBB}$ & BBBD & - & $\mathrm{ABCC}$ & BCCC & B & BDDD & FCM \\
\hline 3 & 109 & $\mathrm{~F}$ & 169924 & BDDD & AAAB & AAAB & BCCC & B & BBBD & - & $\mathrm{ABCC}$ & BCCC & B & BDDD & FCM \\
\hline 4 & 117 & $\mathrm{~F}$ & 169456 & BDDD & AAAB & A & BCCC & B & BBBD & $\mathrm{BBBC}$ & AAAC & $\mathrm{BBBC}$ & B & BDDD & FCM \\
\hline 5 & 117 & $\mathrm{~F}$ & 169457 & BDDD & AAAB & A & BCCC & B & BBBD & - & A & - & B & BDDD & FCM \\
\hline 6 & 117 & $\mathrm{~F}$ & 169458 & BDDD & AAAB & A & BCCC & B & BBBD & - & AAAC & - & B & BDDD & FCM \\
\hline 7 & 117 & $\mathrm{~F}$ & 169459 & BDDD & AAAB & A & BCCC & B & BBBD & $\mathrm{BBBC}$ & AAAC & BCCC & B & BDDD & FCM \\
\hline 8 & 126 & $\mathrm{~F}$ & 169795 & BDDD & AAAB & AAAB & $\mathrm{BBBC}$ & B & BBBD & BBBC & - & BBCC & B & BDDD & FCM \\
\hline 9 & 126 & $\mathrm{~F}$ & 169796 & BDDD & AAAB & AAAB & BCCC & B & BBBD & $\mathrm{BBBC}$ & - & $\mathrm{BBBC}$ & B & BDDD & FCM \\
\hline 10) & 161 & $\mathrm{~F}$ & 166998 & BDDD & AAAB & AAAB & BCCC & B & BBBD & B & - & BCCC & B & BDDD & 1396 \\
\hline 11 & 179 & $\mathrm{~F}$ & 169833 & BDDD & AAAB & AAAB & BCCC & B & BBBD & BBBC & $\mathrm{ABCC}$ & C & B & BDDD & FCM \\
\hline 12 & 192 & $\mathrm{~F}$ & 160377 & BDDD & AAAB & AAAB & BCCC & B & BBBD & BBBC & AAAC & B & B & $\mathrm{D}$ & 1536 \\
\hline 13 & 194 & $\mathrm{~F}$ & 166038 & BDDD & AAAB & AAAB & $\mathrm{BBBC}$ & B & BBBD & $\mathrm{BBBC}$ & AAAC & $\mathrm{BBBC}$ & B & BDDD & 1203 \\
\hline 14 & 203 & $\mathrm{~F}$ & 165987 & BDDD & AAAB & AAAB & BCCC & B & BBBD & BBBC & - & B & B & BDDD & 1089 \\
\hline 15 & 203 & $\mathrm{~F}$ & 166039 & BDDD & AAAB & AAAB & BCCC & B & BBBD & - & ACCC & B & B & BDDD & 1447 \\
\hline 16 & 203 & $\mathrm{~F}$ & 166040 & BDDD & AAAB & AAAB & BCCC & B & BBBD & - & ACCC & B & B & BDDD & 1332 \\
\hline 17 & 203 & $\mathrm{~F}$ & 166041 & BDDD & AAAB & AAAB & BCCC & B & BBBD & - & AAAC & B & B & BDDD & 1317 \\
\hline 18 & 203 & $\mathrm{~F}$ & 166042 & BDDD & AAAB & AAAB & BCCC & B & BBBD & - & AAAC & B & B & BDDD & 1387 \\
\hline 19 & 207 & $\mathrm{~F}$ & 169880 & BDDD & AAAB & A & BCCC & B & BBBD & - & $\mathrm{BBCC}$ & B & B & BDDD & FCM \\
\hline 20) & 207 & $\mathrm{~F}$ & 169881 & BDDD & AAAB & AAAB & BCCC & B & BBBD & - & AAAC & B & B & BDDD & FCM \\
\hline
\end{tabular}

Appendix 2-8: Genotypes of tetraploid (4n) Ambystoma (2) laterale - (2) jeffersonianum (LLJJ) unisexual specimen from site 18 .

\begin{tabular}{|c|c|c|c|c|c|c|c|c|c|c|c|c|c|c|c|}
\hline & \multirow[b]{2}{*}{ Site } & \multirow[b]{2}{*}{ Sex } & \multirow[b]{2}{*}{ AMNH } & \multicolumn{12}{|c|}{ Locus } \\
\hline & & & & Aat-1 & Aat-2 & Idh-1 & Ldh-1 & Ldh-2 & Mdh-1 & Mpi & Pgi & Pgm-1 & Pgm-2 & Sod-1 & blood \\
\hline 1 & 18 & $\mathrm{~F}$ & 153121 & BBDD & B & $\mathrm{AABB}$ & $\mathrm{BBCC}$ & B & BBDD & $\mathrm{C}$ & $\mathrm{AACC}$ & B & B & BBDD & - \\
\hline
\end{tabular}



Complete lists of all issues of the Novitates and the Bulletin are available at World Wide Web site http://library.amnh.org/pubs. Inquire about ordering printed copies via e-mail from scipubs@amnh.org or via standard mail from: American Museum of Natural History, Library-Scientific Publications, Central Park West at 79th St., New York, NY 10024. TEL: (212) 769-5545. FAX: (212) 769-5009. 